\title{
Functional Analysis of Two Major Sperm Tail Proteins \\ Identifies ODF1 as Being Essential for the Tight Linkage of the Sperm Head to the Tail via SPAG4 and ODF2 as A Component of the $\beta$-catenin Destruction Complex
}

\author{
Dissertation \\ for the award of the degree \\ "Doctor rerum naturalium" (Dr. rer. nat.) \\ of the Georg-August-Universität Göttingen \\ within the doctoral program Biology \\ of the Georg-August University School of Science (GAUSS) \\ submitted by \\ Kefei Yang
}

from Liaoning, P. R. China

Göttingen, 2014 


\section{Thesis committee}

Supervisor: $\quad$ Prof. Dr. Sigrid Hoyer-Fender

Department of Developmental Biology,

Johann-Friedrich-Blumenbach-Institute of Zoology and

Anthropology, Georg-August-University of Göttingen

Co-supervisor: Prof. Dr. med. Dr. h. c. Wolfgang Engel

Department of Human Genetics, University Medicine,

Georg-August-University of Göttingen

\section{Extended thesis committee}

Prof. Dr. Detlef Doenecke

Department of Molecular Biology, University Medicine, Georg-August-University of Göttingen

Prof. Dr. Ernst A. Wimmer

Department of Developmental Biology,

Johann-Friedrich-Blumenbach-Institute of Zoology and

Anthropology, Georg-August-University of Göttingen

Prof. Dr. Martin Göpfert

Department of Cellular Neurobiology

Schwann-Schleiden Research Centre

Prof. Dr. Michael Kessel

Department of Developmental Biology

Max Plank Institute for Biophysical Chemistry

Date of the oral examination: 04. 06. 2014 


\section{Declaration}

I declare that this doctoral thesis titled "Functional Analysis of Two Major Sperm Tail

Proteins Identifies ODF1 as Being Essential for the Tight Linkage of the Sperm Head to the Tail via SPAG4 and ODF2 as A Component of the $\beta$-catenin Destruction Complex" was a product of my experimental research work carried out in the Department of Developmental Biology, Georg-August University Göttingen, and that it has not been submitted elsewhere for the award of any degree. Works of other people cited herein have been indicated specifically, or acknowledged by means of completed references. 
Dedication

To my Family 


\section{Acknowledgment}

My sincere gratitude goes to my supervisor, Prof. Dr. Sigrid Hoyer-Fender, for offering me a doctoral position in her group, and then guiding me with her extraordinary intelligence and remarkable professional experience. I thank her for giving me the trust, advice, encouragement and resources necessary to make this study a success. I thank Prof. Dr. med. Dr. h. c. Wolfgang Engel for being the co-supervisor of this work and for critical comments and helpful suggestions during my annual reports.

Special thanks go to Prof. Dr. med. Dr. h. c. Wolfgang Engel for support of animal experiments.

Lot of thanks go to our cooperative partners, Prof. Dr. Andreas Meinhardt of Department of Anatomy and Cell Biology, Justus Liebig University Giessen, for performing electron micrographs (EM) analyses, as well as Dr. Pawel Grzmil (previously: Department of Human Genetics, University Medicine, Georg-August-University of Göttingen; now: Department of Genetics and Evolution, Institute of Zoology, Jagiellonian University, Cracow, Poland) for his help on measurements and analyses of sperm motility. My gratitude also goes to Prof. Dr. Ibrahim. M. Adham, Stephan Wolf and Lea Piontek (Department of Human Genetics, University Medicine, Georg-August-University of Göttingen) for helping us to generate, cultivate and take care of our knock out mice. I also want to thank Dr. Kristine Henningfeld and Dr. Barbara Rust (Department of Developmental Biochemistry, University Medicine, Georg-August-University of Göttingen) for teaching me microinjection technique of Xenopus embryos.

Furthermore, I want to thank my former colleagues Stephanie Schweizer for generation of Odf1 knock out constructs, Bing Zhang for genotyping part of Odf1 knock out mice and Weronika Sura for doing cloning work for generation of Spag4 
constructs. I also want to thank Marco Andreas Tylkowski, Daniela Hüber, and Jasmin Dröge for taking part of ODF2 research. Special thanks go to bachelor students of our group, Luis Haupt, Zahra Basir Kazerouni, Constanza Tapia, Annie Angelique Nono Megaptche and Melek Umay Tüz, for their wonderful help.

To the past and present colleagues / friends Jianwei Li, Christian Ogaugwu, Bing Zhang, Weronika Sura, Van Ahn Dao, Martin Ehrle, Yonggang Hu, Ingrid Curril, Montserrat Torres Oliva, Yuyin Cai, Qiang Wu, Jordi Tomas Roig, I am very grateful for all the help, suggestions and discussions. Members in Department of Developmental Biology are very much appreciated for their help and assistance, especially Beate Preitz, Birgit Rossi, Helma Gries, Katrin Kanbach, Angelika Löffers and Selen Pfändner.

So much appreciated is Dr. Jianwei Li for correcting results part of this thesis.

Lot of thanks go to my friends Jianwei Li, Christian Ogaugwu, Naihang Guo, Bing Zhang, Qiujun Zhan, Kuan Lu, Lin Luo, Narisu Tao, Tselmeg Uriankhai, Yonggang $\mathrm{Hu}$, Jianhong Wu, Zhiyuan Shi, Xinxin Cai, Yuyin Cai, Qiang Wu for their support and making my stay in Göttingen more exciting.

Last but not least, I want to thank my husband Jianfeng Wang and my parents, without their support, understanding and encouragement this thesis would not have been possible. 


\section{Table of Contents}

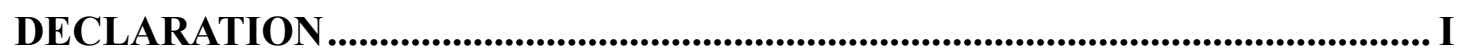

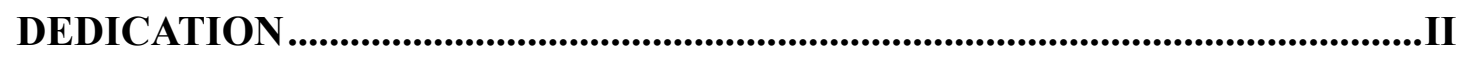

ACKNOWLEDGMENT .............................................................................. III

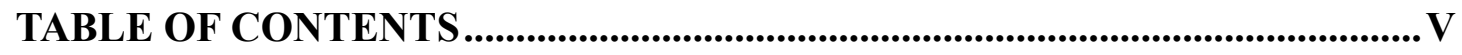

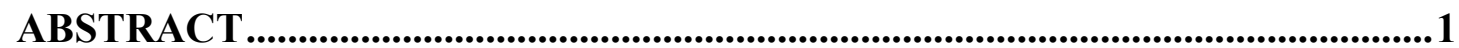

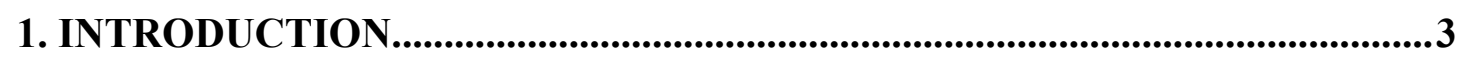

1.1 Mammalian spermatogenesis and male germ cell specific proteins..............................................3

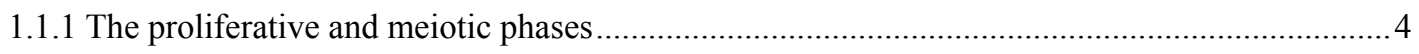

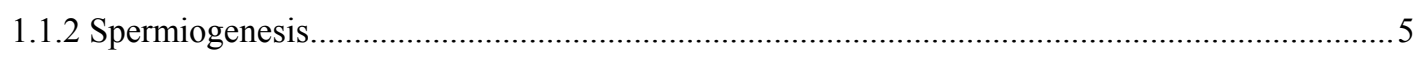

1.1.2.1 Connecting piece of mature spermatozoa ...............................................................

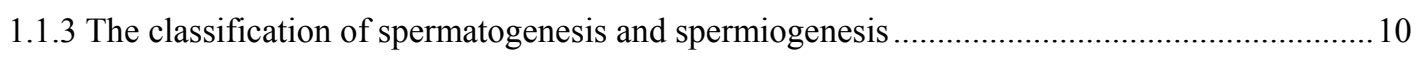

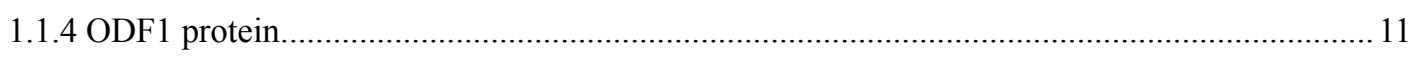

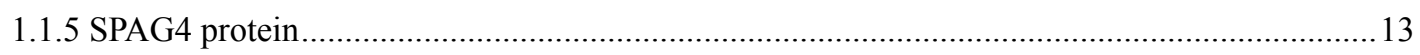

1.2 Centrosome, primary cilium and basal body ..................................................................................14

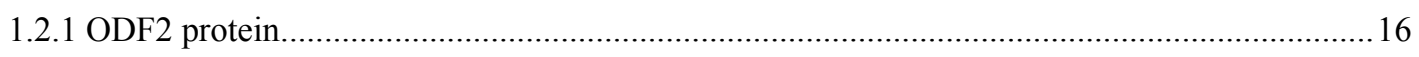

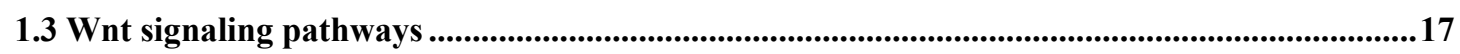

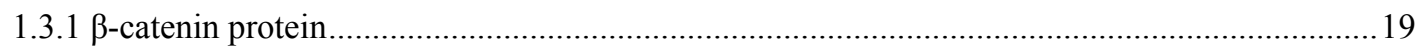

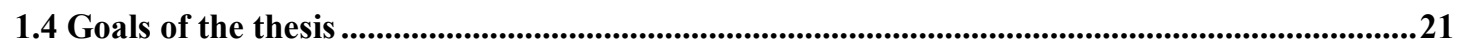

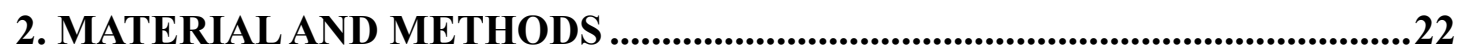

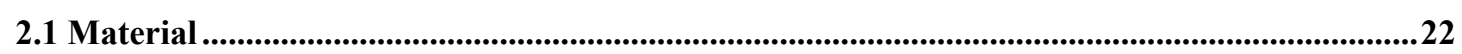

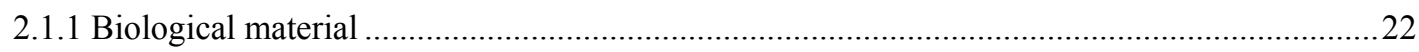

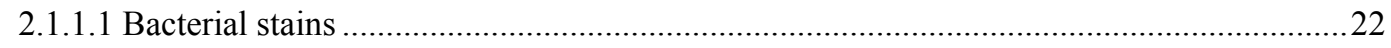

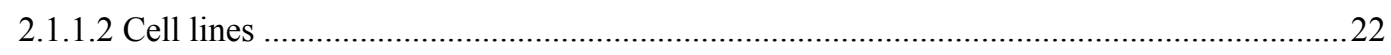

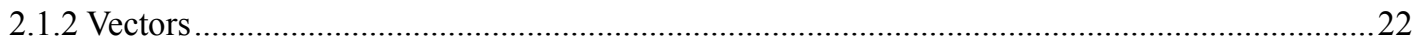

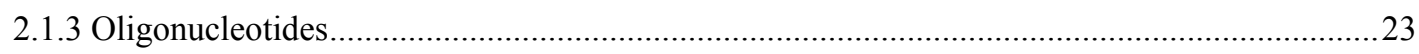

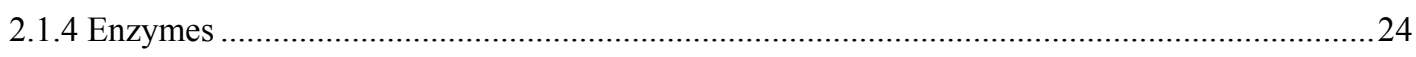

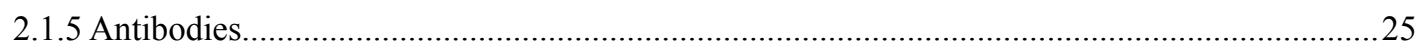

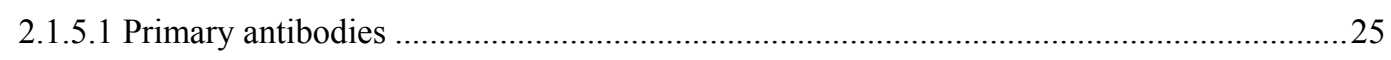

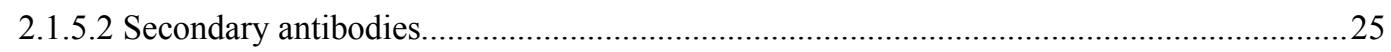

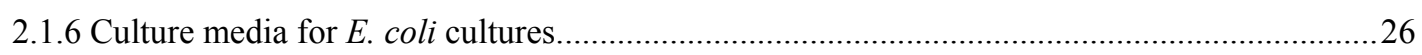


2.1.7 Culture media for eukaryotic cell culture.

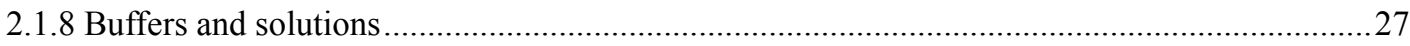

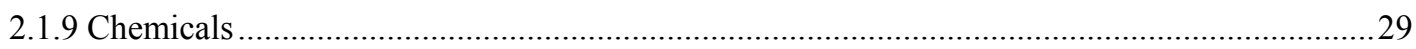

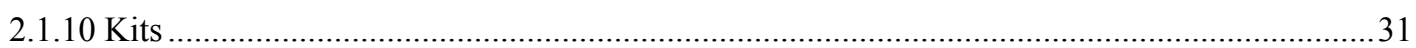

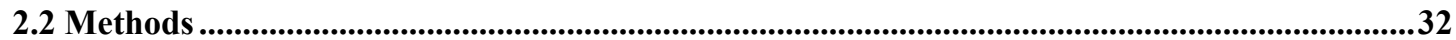

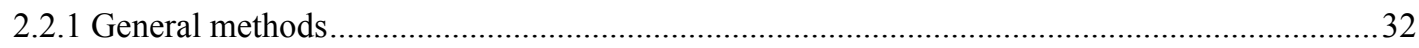

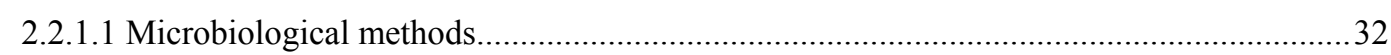

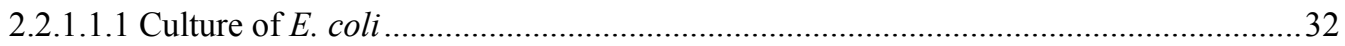

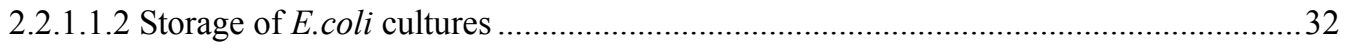

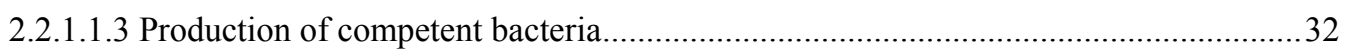

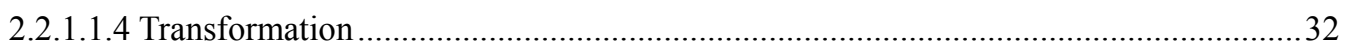

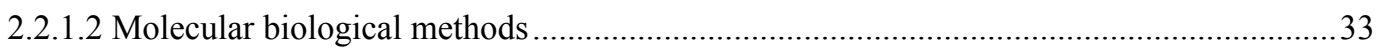

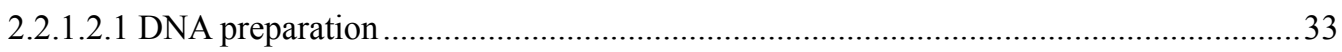

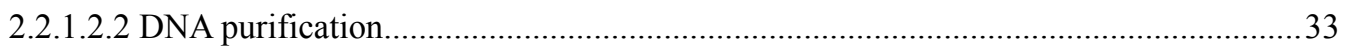

2.2.1.2.3 Measurement of DNA and RNA concentrations ........................................................ 33

2.2.1.2.4 Enzymatic modification of DNA by restriction enzymes........................................ 34

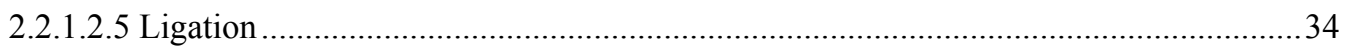

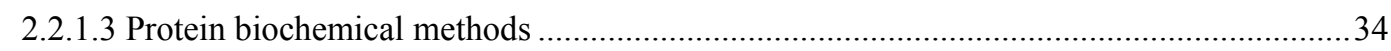

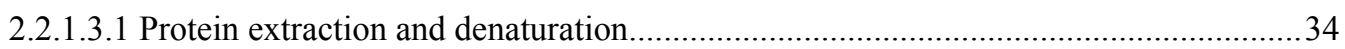

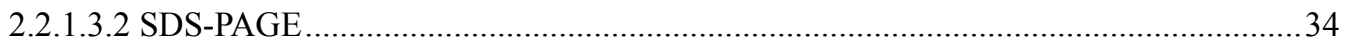

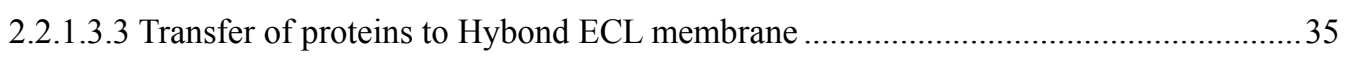

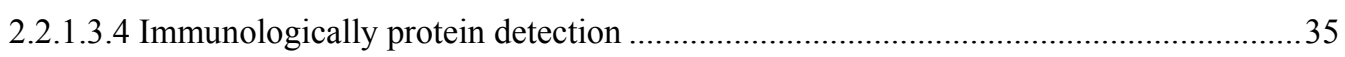

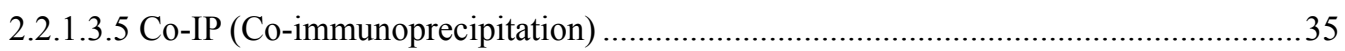

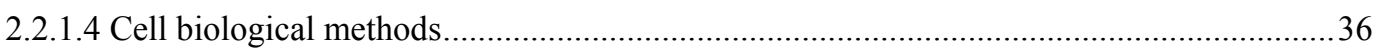

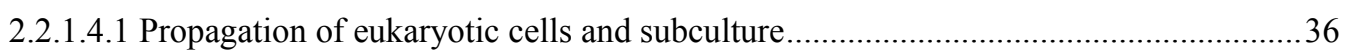

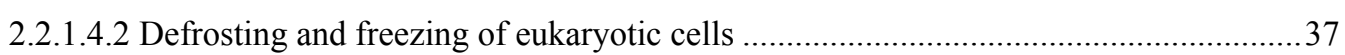

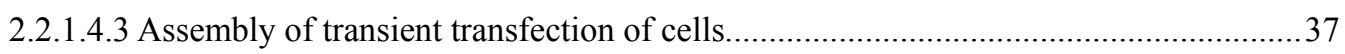

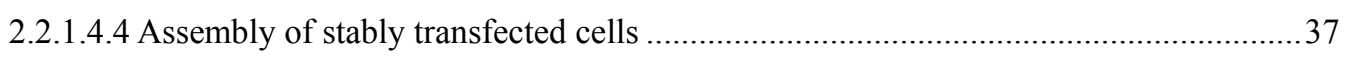

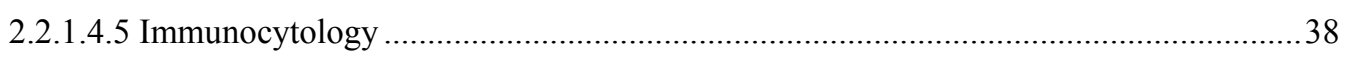

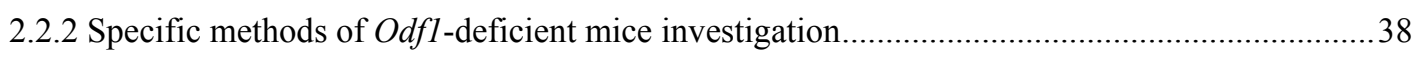

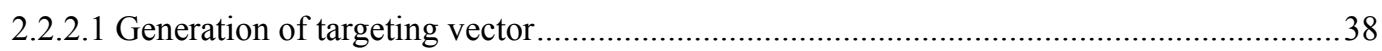

2.2.2.2 Generation of the 3` external probe and Southern blot hybridization .................................39

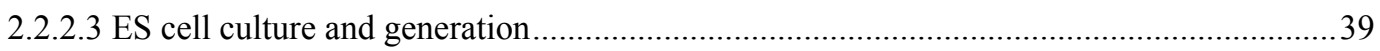

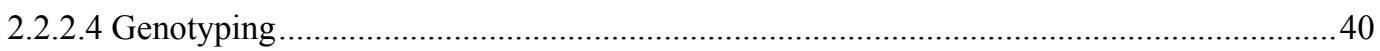

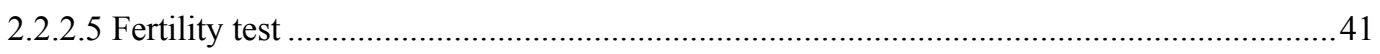

2.2.2.6 Reverse transcriptase PCR on genes of $O d f 1$-deficient mice ......................................... 41

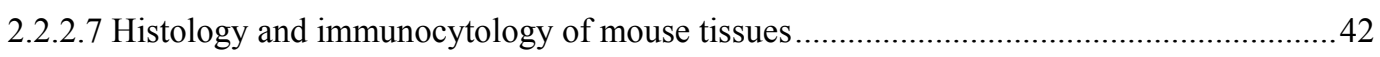

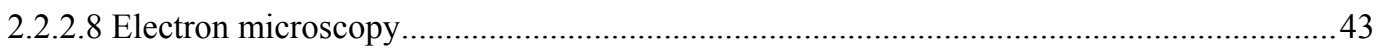

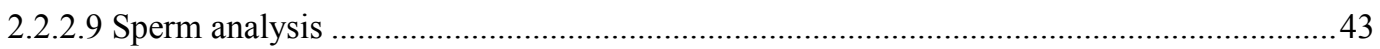

2.2.2.10 In vitro fertilization (IVF) with heterozygous Odfl-deficient males on isogenic

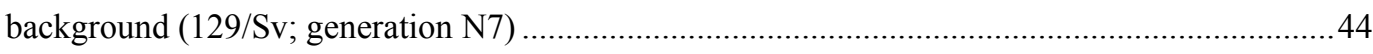

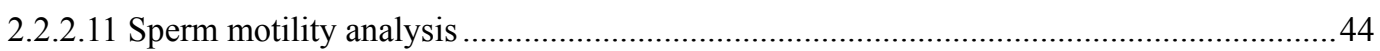

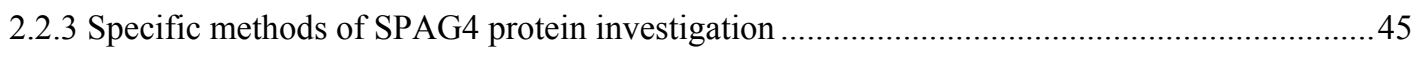

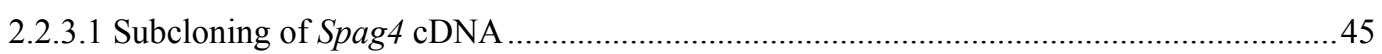




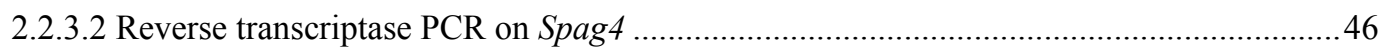

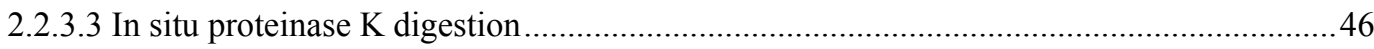

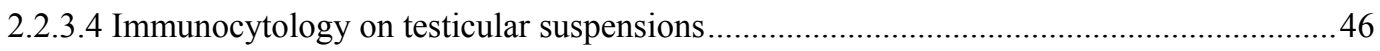

2.2.3.5 Co-IP with transfected cells and testicular tissue ..............................................................4 47

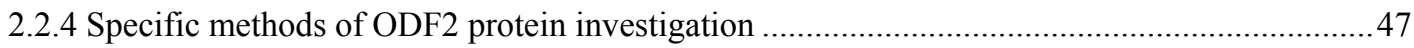

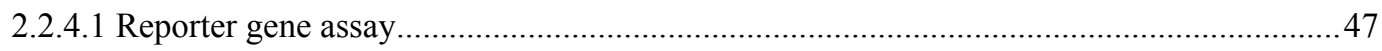

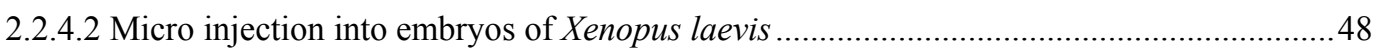

2.2.4.3 Quantitative real time PCR ....................................................................................... 49

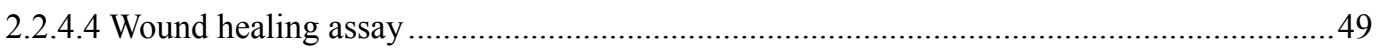

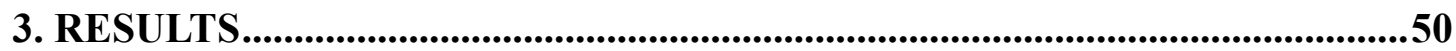

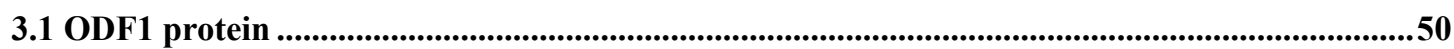

3.1.1 ODF1 protein is essential for tight linkage of sperm head to tail and male fertility in mice .....50

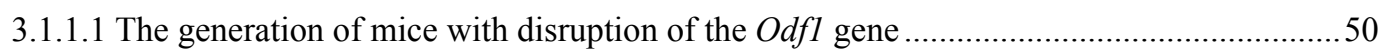

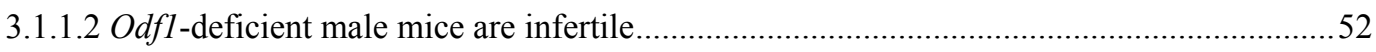

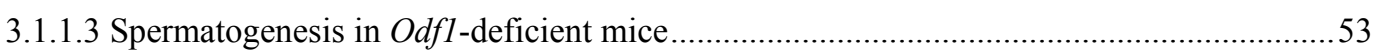

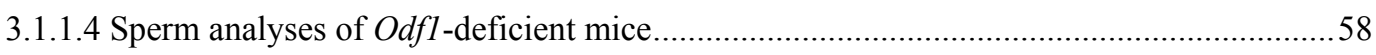

3.1.1.5 Loss of $O d f 1$ affects the ultrastructure of the spermatozoon .............................................61

3.1.2 Odf1-deficient congenic heterozygous mice (129/Sv background) have impaired male fertility

3.1.2.1 Incipient congenic heterozygous male mice are subfertile.........................................64

3.1.2.2 Insemination capacity and sperm parameters are not altered in heterozygous males.........66

3.1.2.3 Fertilization capacity of sperm from heterozygous $O d f 1^{+-}$males is similar to wild-type

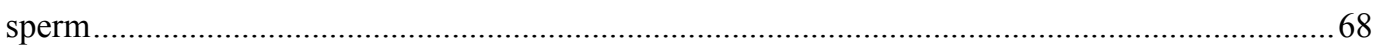

3.1.2.4 Haplo-deficiency of ODF1 increased the distance between nuclear membrane and capitulum.

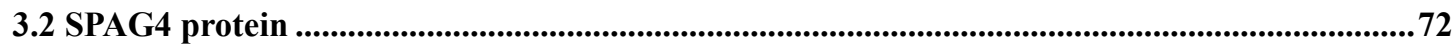

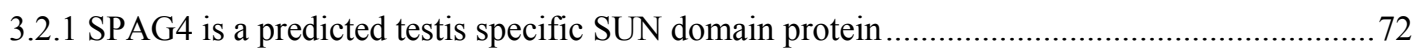

3.2.2 The over-expressed SPAG4 in somatic cells locates to nuclear membrane ............................74

3.2.3 The distribution of endogenous SPAG4 in male germ cells during spermatogenesis ..............79

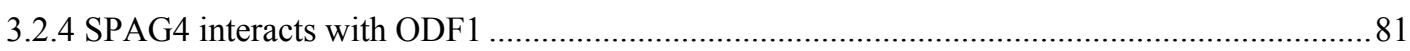

3.2.5 The recruitment of SPAG4 to the posterior pole of elongating spermatids is independent of

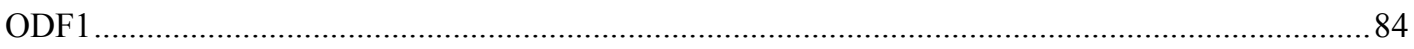

3.2.6 ODF1 is recruited to the nuclear membrane by SPAG4 ....................................................... 86

3.3 ODF2 protein

3.3.1 The influence of centrosomal protein ODF2 on canonical Wnt pathway key protein $\beta$-catenin

3.3.1.1 Ectopic expression of ODF2 promotes the degradation of over-expressed $\beta$-catenin ........88

3.3.1.2 A high ODF2 expression level abets the degradation of endogenous $\beta$-catenin...............91

3.3.2 Investigation of ODF2 protein domains responsible for canonical Wnt pathway inhibition.....93

3.3.3 Centrosomal proteins and cytoskeletal proteins down-regulate the canonical Wnt reporter .....95

3.3.4 The influence of over-expressed ODF2 on target genes of the canonical Wnt pathway ...........98 
3.3.4.1 The over-expression of ODF2 does not obviously affect the activation of canonical Wnt pathway target genes in vivo (Xenopus laevis) .......................................................................98

3.3.4.2 Over-expression of ODF2 inhibits $c-M y c$ transcription ..............................................99

3.3.5 ODF2 over-expression affects the migration of human breast cancer cells............................ 101

3.3.6 ODF2 interacts with Axin1, Axin2 and TAZ proteins individually ..................................... 103

3.3.7 Ectopically expressed ODF2 affects the phosphorylation of Tau .......................................... 106

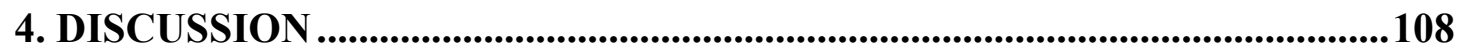

4.1 ODF1 is essential for tight linkage of sperm head to tail and male fertility in mice................108

4.2 Interaction of ODF1 and SPAG4 defines a novel germ cell specific LINC complex involved in sperm head to tail attachment

4.3 ODF2 positively affects degradation of $\beta$-catenin due to influencing its destruction complex

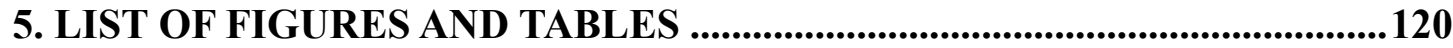

6. REFERENCES....................................................................................................122

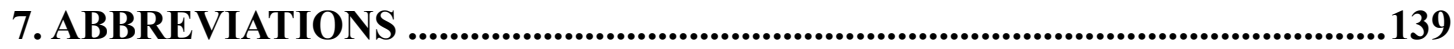

8. SUPPLEMENTAL DATA OF REPORTER GENE ASSAYS ........................143

8.1 The influence of over-expressed ODF2 on $\beta$-catenin activity using reporter gene assays in

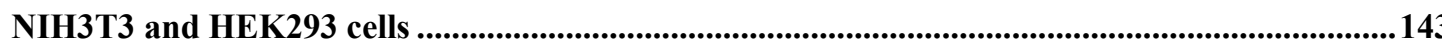

8.2 Canonical Wnt reporter gene assay using Odf2 constructs ....................................................144

8.3 The influence of centrosomal and cytoskeletal proteins on canonical Wnt reporter ...............145

8.4 The influence of centrosomal and cytoskeletal proteins on canonical Wnt reporter with or without nocodazole and taxol

CURRICULUM VITAE 


\begin{abstract}
In the mammalian sperm tail, the outer dense fibers (ODFs) are accessory fibers accompanying the axonemal tubuli doublets on their outer site. The ODFs are composed of at least 14 polypeptides of which only a few have been identified. Among these components, ODF1 interacts with ODF2, and both of them are major proteins of ODFs. Although ODF1 and ODF2 have similar names, which exclusively characterize their location, the fundamental biological functions of them are entirely different.

ODF1 belongs to small heat shock protein (sHSP) family, based on its overall structural features and especially on its conserved $\alpha$-crystallin domain that are characteristic of sHSPs. Therefore, ODF1 was also called HSPB10. The expression of mouse $O d f 1$ is restricted to testis and is first detectable at the round spermatid stage. In addition, immunoelectron microscopy revealed that ODF1 protein is specifically located to the ODFs and connecting piece. To study the role of ODF1 in sperm function, $O d f 1$-deficient mice were generated and shown here that ODF1 is essential for male fertility. Homozygous ODF1-deficient male mice are infertile, whereas heterozygous male mice are fertile but show reduced sperm motility. Odfl-deficient male mice are infertile due to the detachment of the sperm head. Although headless tails are somehow motile, transmission electron microscopy revealed disturbed organization of the mitochondrial sheath, as well as of the outer dense fibers. These results thus suggest that ODF1, besides being involved in the correct arrangement of mitochondrial sheath and outer dense fibers, is essential for rigid junction of sperm head and tail.
\end{abstract}

In somatic cells the cytoskeleton is linked to the nucleoskeleton by nuclear membrane proteins with conserved SUN or KASH domains. SPAG4 is a member of the SUN domain protein family and locates to the nuclear membrane. It directly interacts with ODF1 and recruits ODF1 to the nuclear membrane. Furthermore, SPAG4 is restricted to spermatids and enriched at the posterior part of elongating spermatid nuclei. However, location of SPAG4 is not affected by ODF1 deficiency. Therefore, SPAG4 
and ODF1 seem to be integral components of the head-tail coupling apparatus, and the tight linkage of head to tail in sperm might be mediated by the nuclear membrane protein SPAG4 and its binding to ODF1 in the connecting piece.

Whereas ODF1 is exclusively expressed in spermatids, ODF2, respectively its splice isoform Cenexin, is not only expressed in male germ cells but also localizes generally to the appendages of the mother centriole in somatic cells. As an important centrosomal component, ODF2 is essential for cilia formation and for embryonic development. Since Wnt pathway components are associated with centrosomes and vice versa centrosomal/basal body components have been implicated in modulating Wnt pathways, the relationship of ODF2 and canonical Wnt pathway was investigated. ODF2 inhibited the canonical Wnt pathway. Moreover, ODF2 over-expression repressed transcription of the endogenous Wnt target gene $c-M y c$ and diminished $X W n t 8$-induced secondary axis formation in Xenopus embryos. ODF2 interacted with components of the $\beta$-catenin destruction complex Axin1, Axin2, and $\beta$-catenin, and supported $\beta$-catenin degradation. Therefore, ODF2 most likely is a component of the $\beta$-catenin destruction complex, and this complex might be involved in the regulation of centrosome cohesion via promoting the degradation of $\beta$-catenin. 


\section{Introduction}

\subsection{Mammalian spermatogenesis and male germ cell specific proteins}

The process in which spermatogonia form spermatozoa is known as spermatogenesis. Spermatogenesis occurs within testis. The seminiferous tubules of the testis are the starting point for the progress, where stem cells adjacent to the inner tubule wall divide in a centripetal direction to produce immature sperm and are released into the lumen of tubule (Fig. 1.1). Spermatozoa formed in the testis enter the caput (head) epididymis, progress to the corpus (body), and finally reach the cauda (tail) region, where they are stored. Sperm entering the caput epididymis lack the motility-ability to reach an oocyte. During their transit in the epididymis, sperm mature (Jones, 1999). Final maturation of sperm is completed in the female reproductive tract and called capacitation (Chang, 1951; Austin, 1951).

Spermatogenesis can be divided into three phases based upon functional considerations: (1) the proliferative phase (spermatogonia), in which cells undergo rapid successive divisions; (2) the meiotic phase (spermatocytes), in which genetic material is recombined and segregated; (3) the differentiation or spermiogenic phase (spermatids), in which spermatids thransform into cells structurally equipped to reach and fertilize the oocyte (Russell et al., 1990).

There are many germ-cell specific proteins, which are expressed depending on different sperm development stages. Among them, the expression of mouse ODF1 is restricted to testis and is first detectable at the round spermatid stage. In addition, immunoelectron microscopy revealed that ODF1 protein is specifically located to the outer dense fibers (Burmester and Hoyer-Fender, 1996; Morales et al., 1994). ODF1 therefore seems to be functionally involved in spermatid differentiation and formation of sperm tail. Like ODF1, SPAG4 is exclusively transcribed in round spermatids and translated in elongating spermatids (Shao, et al., 1999). The most works of my study are about ODF1 and SPAG4 proteins, therefore both of them will be introduced later on with more details. 


\subsubsection{The proliferative and meiotic phases}

Reproductive strategies in males of all species command that the cell numbers are increased early in the progress of spermatogenesis. The spermatogonial cell population may fulfill this need. These relatively immature cells undergo numerous mitoses to build a large population of cells that will subsequently undergo meiosis and differentiation to form sperm. There are three types of spermatogonia: stem cell spermatogonia, proliferative spermatogonia, and differentiating spermatogonia. The first two groups are undifferentiated spermatogonia. The stem cell spermatogonia may serve as a potential source of germ cells that can repopulate the seminiferous tubule after damage of the testis. The proliferative and differentiating spermatogonia show a higher mitotic rate and are more susceptible to agents that affect spermatogenesis (Russell et al., 1990).

After the differentiation phase most of mature spermatogonia divide to form the primary spermatocytes (Fig. 1.1). They are characterized by highly condensed chromosomes giving the nucleus a coarse chromatin pattern and an intermediate position in the seminiferous epithelium. Primary spermatocytes go through the first meiotic division divided into prophase (subdividing into preleptotene, leptotene, zygotene, pachytene and diplotene), metaphase, anaphase and telophase and become secondary spermatocytes. The cells quickly proceed through this stage and complete the second meiotic division. Meiosis is the process by which the diploid number of chromosomes present in the stem cells (spermatogonia) is reduced to the haploid number present in mature spermatozoa. The products of the second meiotic division are called spermatids (Fig. 1.1). 


\section{Spermatogenesis}

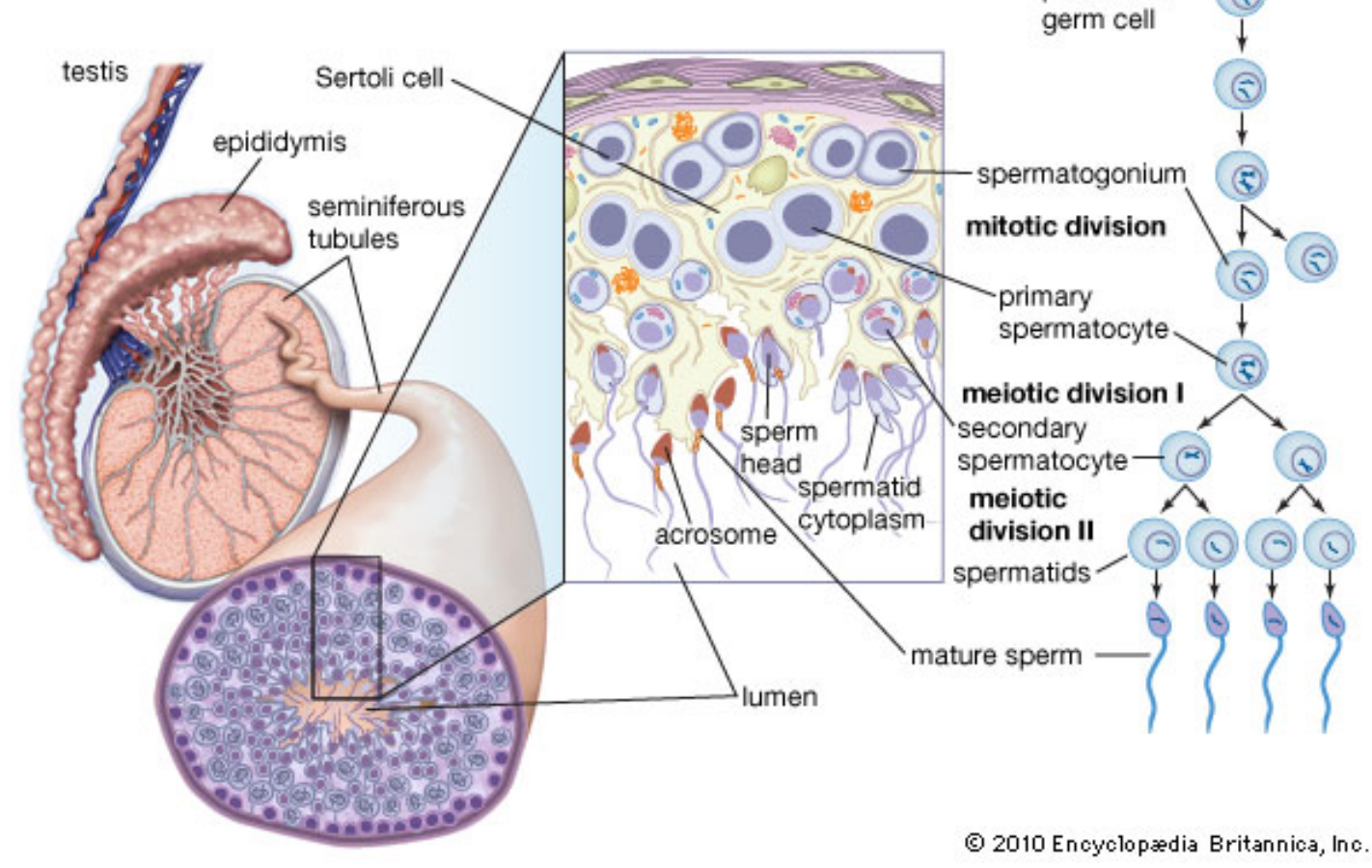

Fig. 1.1: Spermatogenesis. The sperm development occurs in the seminiferous tubules of the testes. The mature spermatogonia divide to form the primary spermatocytes, which go through the first meiotic division and become secondary spermatocytes. After secondary meiotic division, the diploid number of chromosomes present in spermatogonia is reduced to the haploid number present in spermatid. At the end, round spermatids transform into elongated non-motile cells, which are released into the lumen of the seminiferous tubules and subsequently transferred into the epididymis.

\subsubsection{Spermiogenesis}

This phase is the process in which round spermatids transform into elongated non-motile cells. This process occurs without cell division and is one of the most phenomenal cell transformations in the body.

Shortly after the spermatids are formed, the Golgi apparatus is involved in producing small condensing vacuoles (proacrosomal vesicles) containing dense material (proacrosomal granules). One to four proacrosomal granules coalesce within one large 
membrane bounded vesicle containing a single granule called acrosomal vesicle. The round acrosomal vesicle becomes then flat on that side that makes contact with the nuclear surface. The Golgi apparatus is positioned nearby and contributes more and more to the developing acrosome (Fig. 1.2). As time passes, the cell starts to take on an elongate shape, and the nucleus elongates progressively. The cytoplasm is stretched out along the flagellum and the Golgi apparatus moves away from the acrosome and migrates to the caudal aspect of the cell (Russell et al., 1990).

In the young spermatids, the centriole pair migrates to the cell surface, and one of the two centrioles forms an axoneme containing microtubules (9+2 arrangement) that causes the spermatid plasma membrane to protrude from the cell. Then the centrioles pair moves from cell surface to the nucleus and the plasma membrane attached to the centriole folds inwards. After contacting the nucleus, the flagellar centriole indents the nucleus to form an implantation fossa (Fawcett et al., 1969). Later on, the flagellum matures and is divided into mid, principal, and end pieces (Fig. 1.3; Fawcett, 1958 and 1975). Mitochondria are recruited from the cytoplasm to form a helical pattern around the mid piece of the flagellum (Fig. 1.3). Outer dense fibers form both in the mid piece and principal piece (Fig. 1.3; Irons and Clermont, 1982). A fibrous sheath is also formed in the principal piece (Fig. 1.3; Irons and Clermont, 1982).

In most species, up to certain stage, the nucleus of the spermatid is globular. Thereafter, the sperm head of each species achieves its characteristic shape (Fawcett, 1958 and 1975; Fawcett et al., 1971). The mouse sperm head has a sickle-shape like that of the rat (Tang et al., 1982). In the immature sperm head, a cytoskeletal complex named manchette surrounds the nucleus by a sleeve of microtubules (Wolosewick and Bryan, 1977). Spermatid head changes in size and shape during spermiogenesis and becomes hydrodynamically streamlined to move in the fluid environment of the female reproductive tract. To promote spermatids to become smaller and more streamlined, the chromatin is first reorganized and tightly packaged, and second the cytoplasm is eliminated during spermatid elongation (Sprando and Rusell, 1987). Thereafter, some cytoplasm is eliminated just before sperm release by minute structures (tubulobulbar complexes) (Russell and Clermont, 1976; Russell, 1979; 
Russell and Malone, 1980). Finally, cytoplasmic package (residual body) is formed at sperm release and pinched off from the spermatid to reduce one-fourth of the volume of the spermatids original size (Sprando and Rusell, 1987; Fawcett and Phillips, 1969; Russell, 1984). These cytoplasmic fragments are phagocytized and digested by the Sertoli cell (Kerr and de Kretser, 1974). After cytoplasmic elimination, a small amount of cytoplasm (cytoplasmic droplet) remains around the neck of the spermatid.

\section{Spermiogenesis}

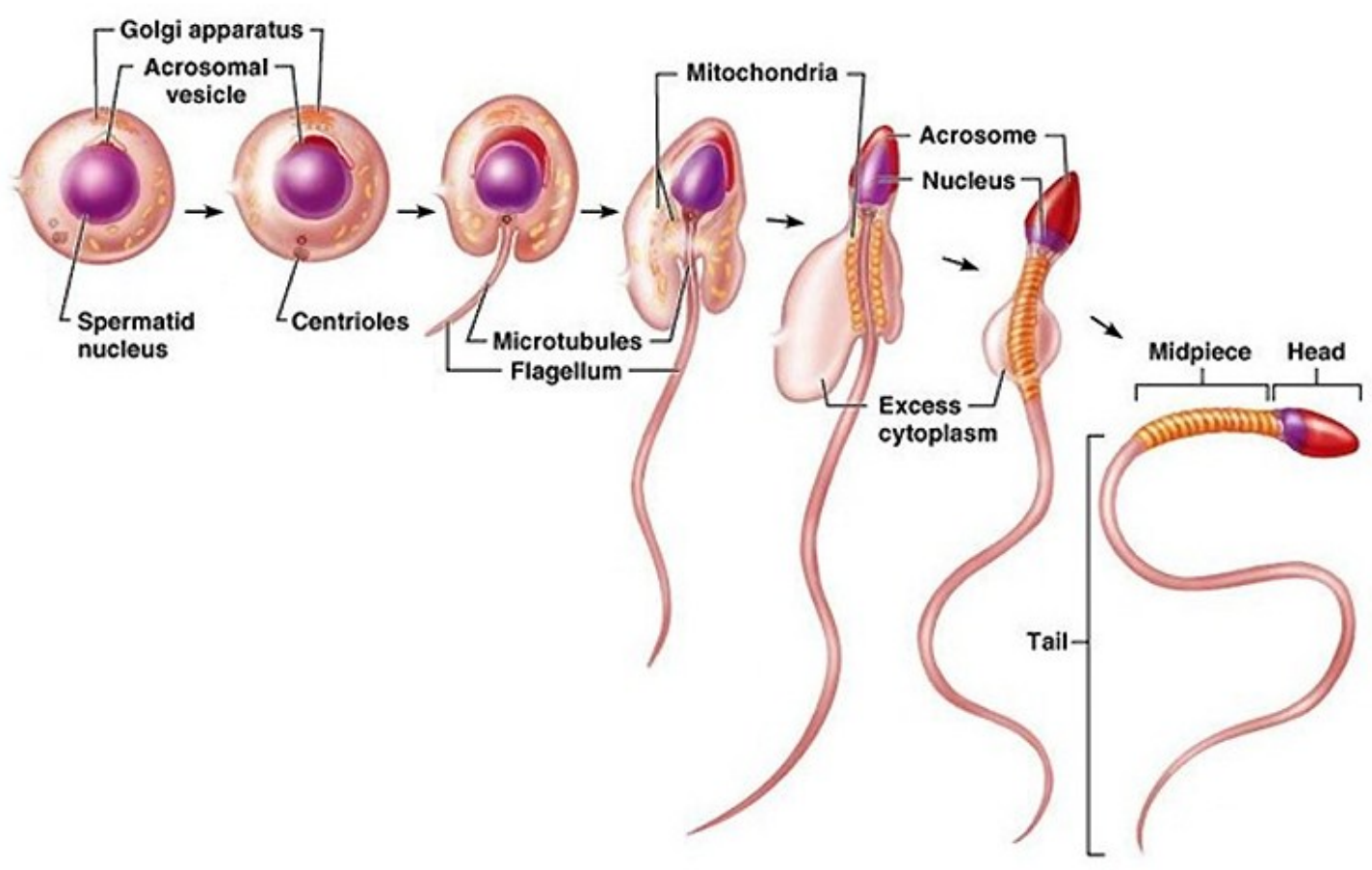

Fig. 1.2: Schematic drawing of spermiogenesis, here shown for human. During spermiogenesis, the spermatid transforms from spherical form to an elongate shape. The centrioles go to the cell surface and one of them forms the axoneme, and afterwards, the flagellum is formed as sperm tail. Mitochondria are arranged to form a helical matrix surrounding the mid piece of the flagellum. The Golgi apparatus is involved in producing proacrosomal vesicles and contributes more and more to the developing acrosome. Some cytoplasm is eliminated and cytoplasmic package is formed, and pinched off from the spermatid. (The picture is from (C) 2004 Pearson, inc., publishing as Benjamin Cummings). 
The end products of spermiogenesis are spermatozoa. Spermatozoa consist of a head and a tail (Fig. 1.3). The head comprises the nucleus and the acrosome attached at its cranial pole. At the caudal end the spermatozoan nucleus is anchored to the tail, which is responsible for sperm motility and movement towards the oocyte. In order to propel the spermatozoan head and to circumvent its disconnection from the sperm tail, sperm head and tail must be tightly linked to each other. Firm coupling is achieved by a sophisticated structure, the connecting piece.

\section{The spermatozoon}

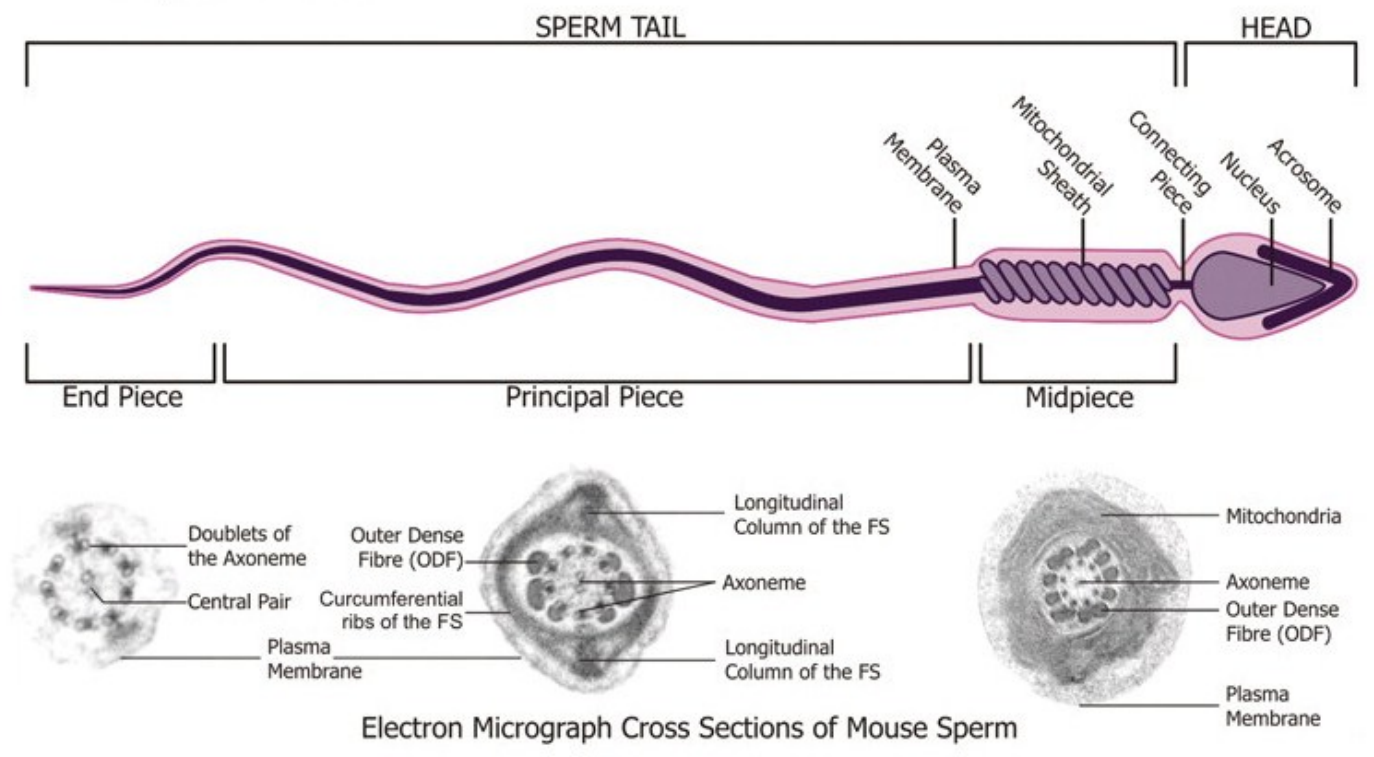

Fig. 1.3: The Spermatozoon. The spermatozoon is constituted of two main regions, the head and the tail. The anterior portion of the head is covered by the acrosomal cap and the head is joined to the tail by the connecting piece. The tail is divided into three regions: the midpiece; principal piece; and the end-piece. The electron micrographs showing cross-sections (not to scale) of each region highlight the main components of the tail structure: the axoneme; outer dense fibers (ODFs); and the mitochondrial sheath (midpiece) and fibrous sheath (FS) (principal piece). The end-piece consists solely of the axoneme and plasma membrane (Borg et al., 2010). 


\subsubsection{Connecting piece of mature spermatozoa}

The connecting piece is organized into nine longitudinal segmented columns that fuse cranially to form the capitulum (Fig. 1.4). The capitulum is a dense fibrous plate-like structure that conforms to the shape of the implantation fossa (Toshimori, 1998). The capitulum is the counterpart of the basal plate, a dense structure that lines the outer nuclear membrane at the implantation fossa. The interspace between the two membranes of the nuclear envelope in this region contains a regular array of periodic densities $6 \mathrm{~nm}$ wide and $6 \mathrm{~nm}$ apart (Fawcett, 1975). Freeze-fracture studies indicate that the membrane of the nuclear envelope at the implantation fossa contains a dense population of large and regularly spaced intramembranous particles surrounding a central particle-free region (Fried and Fawcett, 1974). The narrow region between capitulum and basal plate is traversed with fine filaments, these filaments are presumably responsible for attaching the capitulum of the flagellum to the basal plate of the head (Pedersen, 1972; Stackpole and Devorkin, 1974). Extending posteriorly from the capitulum two major and five minor segmented columns of 1-2 $\mu \mathrm{m}$ in length are usually found (Fig. 1.4). These two major columns split into two columns each and along with the other five columns, fuse to the nine outer dense fibers extending throughout the middle piece and principal piece of the flagellum. However, the segmented columns and the outer dense fibers have different origins, and the continuity between them develops late in spermiogenesis (Fawcett et al., 1969; Zamboni and Stefanini, 1971). The segmented columns of the connecting piece are cross-striated, with a typical periodicity of $6.65 \mathrm{~nm}$ between segments. Each segment has nine or ten horizontal bands (Fawcett et al., 1969).

The tight coupling between sperm head and tail is essential for successful fertilization. Impaired formation of intimate connection between head and tail causes increased instability of head-tail junction eventually resulting in acephalic spermatozoa and human male infertility (Chemes et al., 1999; chemes, 2012). Although well characterized ultrastructurally, the molecular components responsible for the formation of the connecting piece and the tight linkage of sperm head to tail are 
scarcely known.

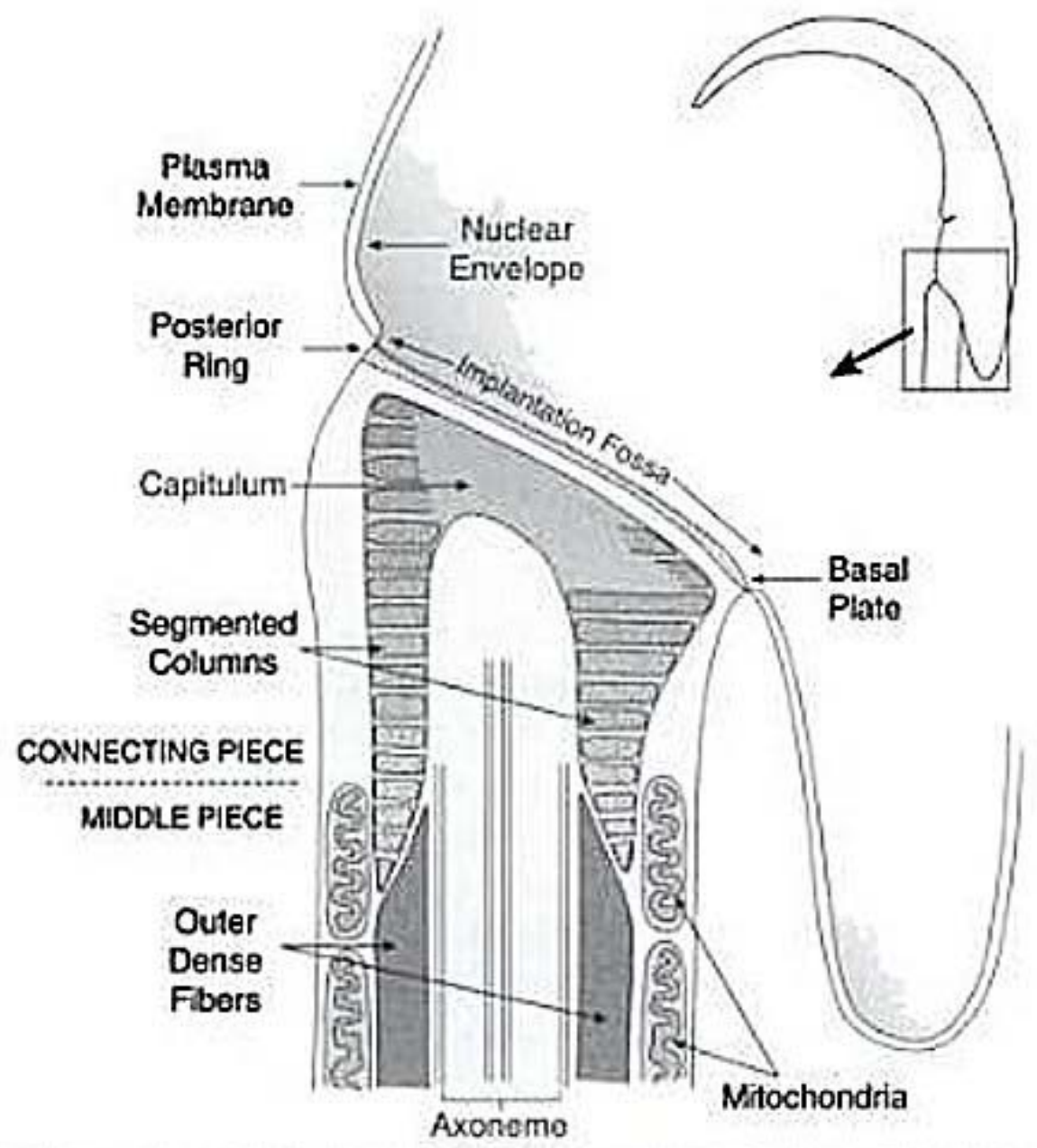

Fig. 1.4: Enlargement of the connecting piece of mouse sperm tail. The capitulum is the counterpart of the basal plate. At their caudal ends the segmented columns pass into the outer dense fibers (ODFs) of the sperm tail (Knobil and Neill's physiology of reproduction, 3. edition, 2006).

\subsubsection{The classification of spermatogenesis and spermiogenesis}

In seminiferous tubules, the progressing spermatogenesis is grouped into stages. A stage is a defined association of germ cell types at particular phases of development in cross-sectioned tubules, and each cell type of the stage is morphologically integrated with the others in its developmental processes (Russell et al., 1990). By convention, 
each stage is designated by a Roman numeral (Fig. 1.5). In mice, there are 12 designated stages (Fig. 1.5).

Due to the morphological changes of spermatid during spermiogenesis, spermiogenesis may be divided into various developmental steps. It bases on the form and shape of the acrosome, the spermatid head shape and the degree of chromatin condensation. Steps of spermiogenesis are provided with Arabic number designations (Russell et al., 1990). In mice, there are 16 steps in spermiogenesis (Fig. 1.5).

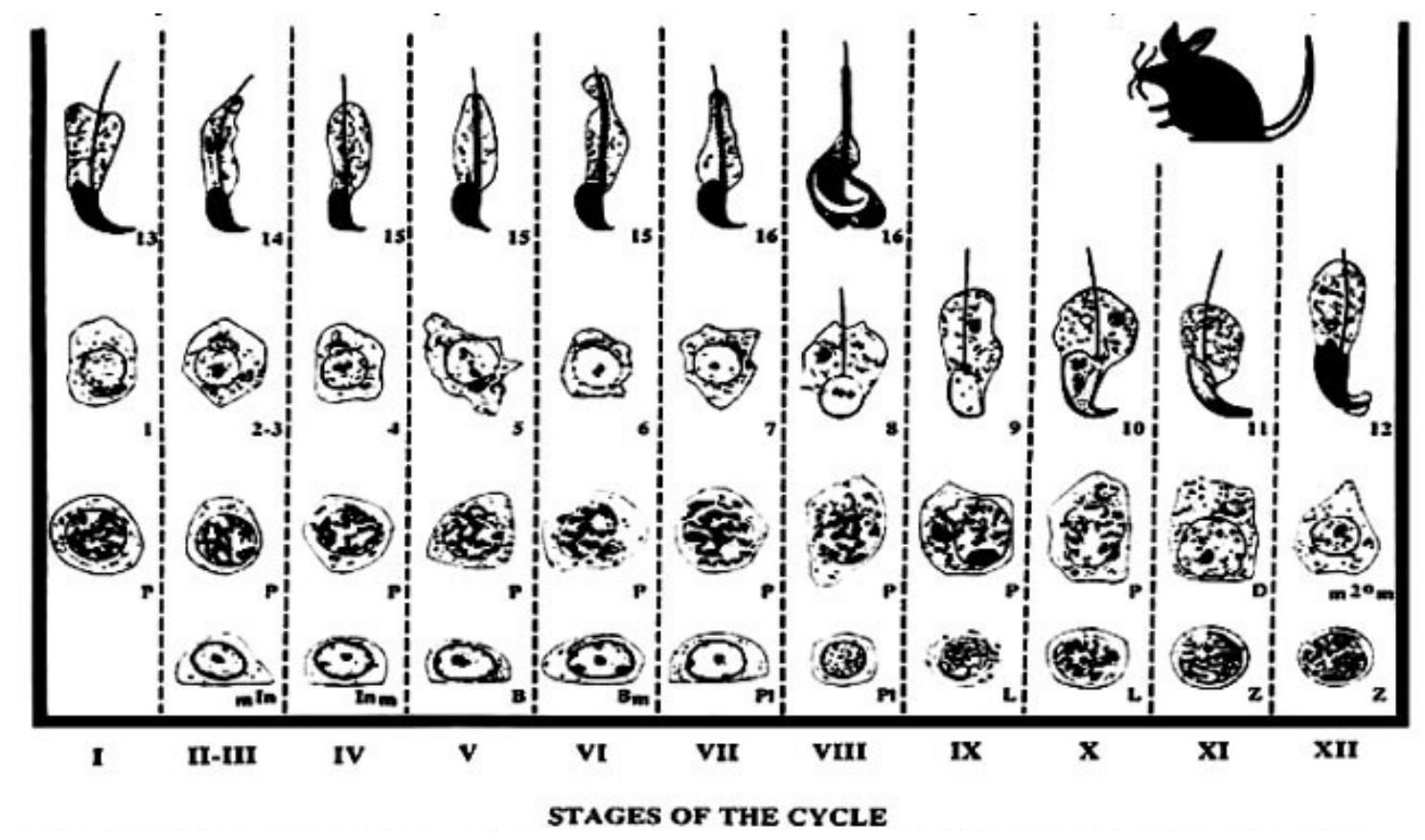

Fig. 1.5 Stages of spermatogenesis and steps of spermiogenesis in mice (Russell et al., 1990).

\subsubsection{ODF1 protein}

ODF1 is the major protein of mammalian sperm tail outer dense fibers (ODFs), which are cytoskeletal structures specifically found in the sperm tails of vertebrates but not in eukaryotic cilia and flagella in general (Fawcett, 1975). ODFs are accessory fibers accompanying the axonemal tubuli doublets on their outer side and are therefore present ninefold. They are composed of more than a dozen different proteins of which only a few have been identified (Oko, 1988; Petersen et al., 1999; Vera et al., 1984). 
Although not involved in active motility, ODFs seem to be important for the stability and the elastic recoil of the sperm tail, as well as for support of the flagellar beat (Baltz et al., 1990; Lindemann, 1996). Impaired development of the ODFs has been described as a major cause of tail abnormalities in infertile men, indicating an important function in sperm motility and/or morphology (Haidl et al., 1991).

ODF1 is the most recent protein assigned to small heat shock proteins (sHSPs), based on its overall structural features and especially on its conserved $\alpha$-crystallin domain that is characteristic of sHSPs (Fontaine et al., 2003). Therefore, ODF1 was also called HSPB10 (Fontaine et al., 2003). Heat shock proteins play an essential role as molecular chaperones by preventing the aggregation of misfolded proteins and have strong cytoprotective effects. A structurally divergent group within the molecular chaperone family is constituted by sHSPs. The super-family of sHSPs comprises 10 proteins with molecular masses ranging from 12 to $43 \mathrm{kDa}$. Their most pronounced structural feature is the conserved $\alpha$-crystallin domain of 80 to 100 amino acids. sHSPs interact with a lot of essential cellular structures. Hence, sHSPs seem to be implicated in a wide variety of human diseases (Hishiya et al., 2008; Lanneau et al., 2008; Sun et al., 2005).

The Odf1 gene, which is a single copy gene located in mice to chromosome 15 region B2-C, as well as its encoded protein, are highly conserved in evolution (Gastmann et al., 1993; Oko, 1988), indicating an important function in sperm motility and/or morphology.

ODF1 has a molecular mass of about $27 \mathrm{kDa}$ with high content of cysteine (between $13 \%$ and $17 \%$ ) and proline (up to $10 \%$ ), capably forming disulfides (Burfeind and Hoyer-Fender, 1991; Burfeind et al., 1993; Gastmann et al., 1993; Hoyer-Fender et al., 1995; Morales et al., 1994; Van der Hoorn et al., 1990). The high cysteine content of ODF1 may provide free sulphydryl groups for the retention of zinc ions that are enriched in the sperm tail (Calvin et al., 1975). Cysteine and proline are mainly found in the C-terminal end of ODF1 as a repetitive tripeptide motif of C-X-P. Although variability of C-X-P repeat frequencies has been reported, it did not affect male fertility in humans (Hofferbert et al., 1993). 
ODF1 is a self-interacting protein but its self-association is weak (Shao and Van der Hoorn, 1996). Moreover, several binding partners were also identified over years, including ODF2. Interaction between ODF1 and ODF2 was proven in a yeast two-hybrid assay and was shown to depend on their leucine-zipper motif (Shao et al., 1997). The scaffold of the ODFs may thus be structured by the two main ODF proteins, ODF1 and ODF2 (Petersen et al., 1999). Other ODF1 interacting proteins are SPAG4, KLC3 (kinesin light chain 3), OIP1 (ODF1-interacting protein), and SPAG5 (Bhullar et al., 2003; Fitzgerald et al., 2006; Shao et al., 1999; Zarsky et al., 2003). SPAG4 is located in the transient manchette and associated with the axoneme in elongating spermatids and epididymal sperm. It binds to ODF1, but not to ODF2 (Shao et al., 1999). SPAG5 is found in rat elongated spermatids and epididymal sperm. SPAG5 associates with ODFs, but not with the axonemal microtubules (Fitzgerald et al., 2006). OIP1 is a member of the RING finger family that are often E3 ubiquitin protein ligases. OIP1 binds to the conserved C-X-P motif in the C-terminal end of ODF1 (Zarsky et al., 2003), and ODF1-OIP1 interaction is enhanced by Cdk5/p35-mediated ODF1 phosphorylation (Rosales et al., 2008). In addition to being components of the ODFs, ODF1, ODF2, and ODF1 interacting protein SPAG4 have all been located to the manchette, a transient microtubular structure that seems to be important for the shaping of the sperm nucleus and the development of the sperm tail (Kierszenbaum et al., 2002).

\subsubsection{SPAG4 protein}

SPAG4 is a member of the SUN domain proteins and locates to the nuclear membrane. Its expression is restricted to spermatids and it is enriched at the posterior part of elongating spermatids (Yang, et al., in preparation).

SUN proteins were discovered by molecular analysis of Caenorhabditis elegans unc-84 that affected nuclear migration (Horvitz and Sulston, 1980; Sulston and Horvitz, 1981). Sequence comparison revealed conservation of the C-terminal region 
of UNC-84 with the C-termini of Schizosaccharomyces pombe Sad-1 and two human proteins, SUN1 and SUN2 (Malone et al., 1999). This region that consists of $\sim 175$ amino acids was hence named SUN domain (for sad-1 and unc-84). SUN domain proteins have at least one transmembrane domain that localizes to the nuclear membrane. Altogether, SUN domain proteins are most likely located in the inner nuclear membrane with their SUN domains in the perinuclear space whereas their $\mathrm{N}$-terminal regions extend into the nucleoplasm (Malone et al., 1999; Dreger et al., 2001; Schirmer et al., 2003; Hodzic et al., 2004; Liu et al., 2007). Due to the presence of short coiled-coil regions SUN domain proteins are able to form dimers or multimers (Crisp et al., 2006; Haque et al., 2006; Lu et al., 2008; Malone et al., 1999). The N-terminal nucleoplasmic regions of SUN1 and SUN2 interact with A- and B-type lamins whereas the conserved SUN domain itself interacts with the conserved KASH domain found in the outer nuclear membrane proteins Klarsicht, ANC-1, SYNE-1/Nesprin, and MSP-300 (hence termed KASH for Klarsicht/Anc-1/Syne-1 homology) (Hodzic et al., 2004; Padmakumar et al., 2005; Haque et al., 2006; McGhee et al., 2006; Tzur et al., 2006; Crisp et al., 2006).

KASH and SUN domain proteins thus bridge the nuclear envelope to function as a linker of the nucleoskeleton to the cytoskeleton complexes (Crisp et al., 2006; Haque et al., 2006; McGhee et al., 2006; Razafsky and Hodzic, 2009; Starr and Fridolfsson, 2010). In mammals, so far, at least five SUN domain proteins are present of which SUN1 and SUN2 are widely expressed whereas SUN3/SUNC1, SUN4/SPAG4, and SUN5/SPAG4L are largely restricted to testis. It has been reported that SPAG4 locates in the manchette and the axoneme, binds to ODF1, and is switched on in neoplastic tissues (Shao et al., 1999; Kennedy et al., 2004).

\subsection{Centrosome, primary cilium and basal body}

The centrosome is located at the cell center close to the cell nucleus. It is the major microtubule-organizing center (MTOC) of the eukaryotic cell and generates the 
mitotic spindle during mitosis. The centrosome consists of the mother centriole, which is characterized by the presence of distal and subdistal appendages, and the daughter centriole (Fig. 1.6 A). Centrioles are mainly built from $\alpha / \beta$ tubulin dimers and are surrounded by pericentriolar material (PCM) (Kellogg et al., 1994; Stearns and Winey, 1997; Zimmermann et al., 1999; Bornens, 2002). In animal cells, centrosomes duplicate once per cell cycle, strictly dictated by cell cycle control mechanism. In $\mathrm{S}$ phase, the new centrioles start to form from the original mother and daughter centrioles. These procentrioles elongate through S to G2 phases and then the original daughter centriole acquires appendage proteins in late G2 / early M phase to become a new mother centriole (Fig. 1.6 B).

In eukaryotic cells, primary cilia are anchored to the cell by the basal bodies, which develop from the mother centriole of the centrosome (Wheatley et al., 1996). As essential sensory organelles they project from the surface of cells (Huangfu et al., 2003; Pazour and Witman, 2003; Corbit et al., 2005; Haycraft et al., 2005; Huangfu and Anderson, 2005; May et al., 2005; Pan et al., 2005; Schneider et al., 2005; Michaud and Yoder, 2006). They are generally immotile having nine peripheral doublet microtubules. Primary cilia are found singly on cells whereas motile cilia with additional two central microtubules occur in groups. Primary cilia exist on most epithelial and stromal cells throughout the mammalian body. So far, it has been found that primary cilia are involved in several signaling pathways essential for growth and differentiation, including the Hedgehog (Hh), Wnt, and PDGF pathways (Eggenschwiler and Anderson, 2007; Berbari et al., 2009).

The basal body is converted from the mother centriole. When the cell leaves mitosis and enters G0 phase, the mother centriole associates with ciliary vesicles that cap its distal end (Sorokin, 1962), migrates to the cell surface and subsequently docks at the plasma membrane. In the transition zone, the axonemal doublets of the primary cilium are shifted from triplet microtubules in the basal body, and the outer doublet microtubules are connected to the ciliary membrane with Y-shaped bridges, the transition fibers. Transition fibers and basal feet originate from distal and subdistal appendages of the mother centriole, respectively (Fig. 1.6 C). Transition fibers (distal 
appendages) function to anchor the basal body to the membrane in the transition zone (Anderson, 1972). In addition, transition fibers (distal appendages) might also be important for the docking of intraflagellar transport (IFT) proteins to promote ciliogenesis (Deane et al., 2001). Striated rootlets extend from the proximal end of the basal body into the cytoplasm supporting the cilium (Tachi et al., 1974).

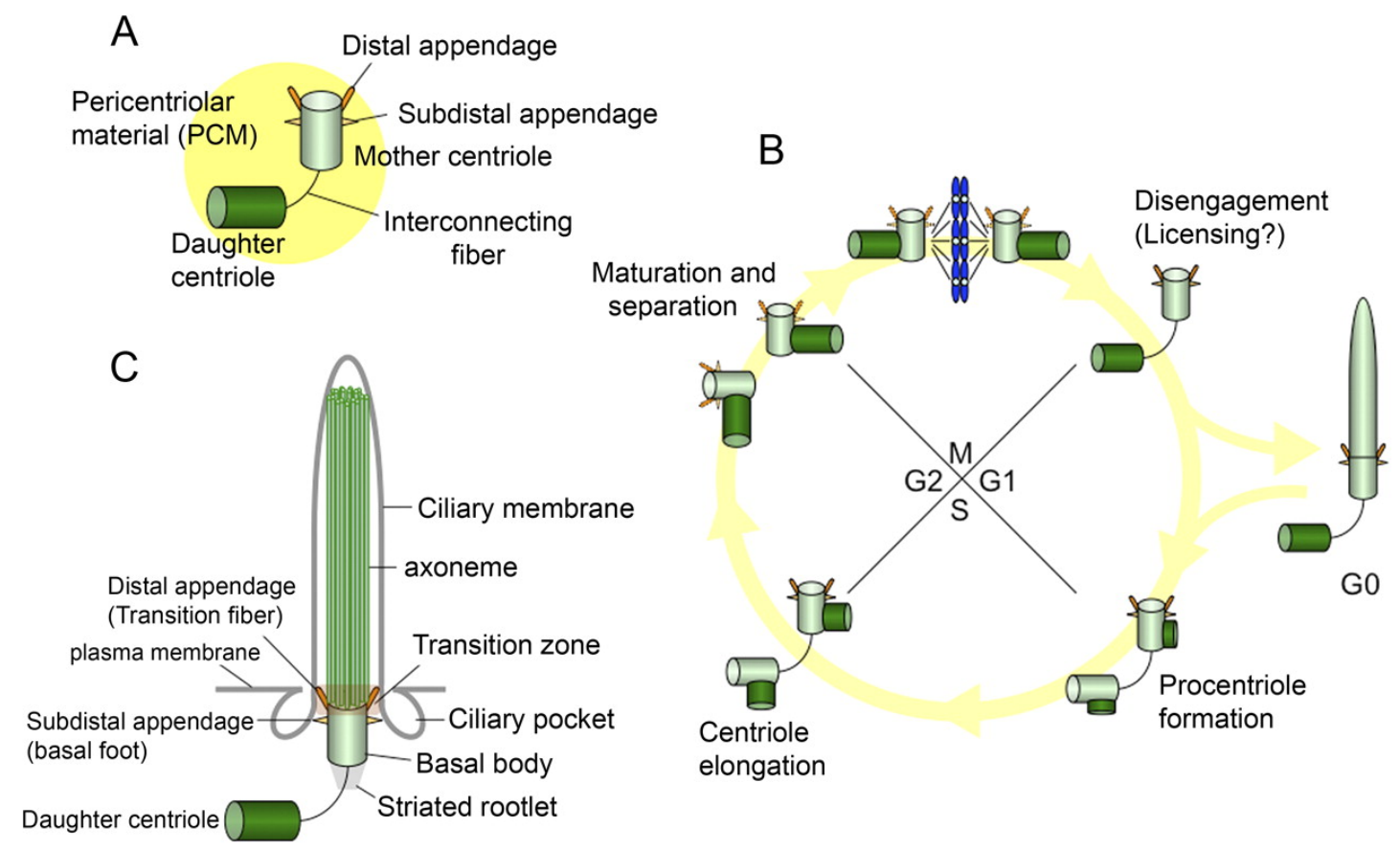

Fig. 1.6: Centrosome, primary cilium and cell cycle. (A), the structure of centrosome. (B), the centrosome duplication during cell cycle. When the cell leaves G0 phase, the primary cilium disappears and the basal body becomes the centrosome generating the mitotic spindle with major impacts on chromosome segregation and cytokinesis. (C), the structure of primary cilium. In G0 phase the centrosome goes to the cell surface and the mother centriole becomes the basal body. The primary cilium is formed from the basal body (review of Kobayashi and Dynlacht, 2011).

\subsubsection{ODF2 protein}

Originally, ODF2 was found in the mammalian sperm tail and is the major component of the outer dense fibers (ODFs) (Brohmann et al., 1997; Shao et al., 1997; Turner et 
al., 1997; Hoyer-Fender et al., 1998; Schalles et al., 1998; Petersen et al., 1999). Comparing to ODF1, which is another important protein of the outer dense fibers and restricted to spermatids, ODF2 generally localizes also to the appendages of the mother centriole in somatic cells (Nakagawa et al., 2001; Hoyer-Fender et al., 2003; Donkor et al., 2004). More precisely, Cenexin is confirmed as an isoform of ODF2 which is constantly expressed in all tissues whereas ODF2 was upregulated in testes (Hüber and Hoyer-Fender, 2007). Both proteins differ by a peptide of 42 amino acids at the N-terminal region which is present in Cenexin and absent in ODF2. This cenexin insertion is encoded by exon $3 b$ and starts with codons for the amino acids MKDR. In the C-terminal region two leucine zipper motifs are present which are responsible for interaction with ODF1 (Shao et al., 1997). According to a secondary structure prediction (Lupas et al., 1991), ODF2 has a coiled-coil structure on its C-terminal end. The contribution of this coiled-coil structure most probably relies on promoting self-association and the formation of higher-order structures. Alternative splicing of ODF2/Cenexin transcripts encodes isoforms with masses between 70 and $100 \mathrm{kDa}$. At the C-terminal end human and rat Cenexin isoforms have an extension of $\sim 150$ amino acids that is not present in ODF2 isoforms (Soung et al., 2006; Rivkin et al., 2008).

\subsection{Wnt signaling pathways}

Wnt signaling pathways play crucial roles in cell proliferation, cell fate specification and morphogenesis in early embryos. They consist of proteins which can pass the signals from the cellular outside via cell surface receptors to the cellular inside. Three Wnt signaling pathways have been characterized: the canonical Wnt / $\beta$-catenin pathway, the planar cell polarity pathway (PCP pathway) and the calcium pathway. The PCP pathway is one of the non-canonical pathways ( $\beta$-catenin-independent pathways), controlling cell polarity in the plane of epithelial tissues in Drosophila (Lawrence et al., 2007; Seifert and Mlodzik, 2007). The other non-canonical pathway, 
the calcium pathway, can help to regulate calcium release from the endoplasmic reticulum (ER) in order to control intracellular calcium levels and this pathway is dependent on G-proteins (Kohn and Moon, 2005; Slusarski and Pelegri 2007).

The canonical Wnt pathway is a $\beta$-catenin dependent Wnt pathway therefore also called $\beta$-catenin pathway. In this pathway, $\beta$-catenin as a transcriptional co-activator plays a key role in transmitting extracellular signals for the activation of target genes. In the absence of Wnt, $\beta$-catenin is constitutively phosphorylated by a destruction complex consisting of Axin, Dvl, APC and GSK3 $\beta$ (glycogen synthase kinase 3 $\beta$ ). The phosphorylated $\beta$-catenin is subsequently ubiquitinated by the E3 ligase $\beta$-TrCP ( $\beta$ - transducin repeat-containing protein) and degraded in the proteasome. This degradation results in a low cellular $\beta$-catenin level (Fig. 1.7 A). In contrast, in the presence of Wnt, Wnt ligand binds to its receptor Fz (Frizzled) and co-receptor LRP6. Then the activated Frizzled receptors recruit the cytoplasmic protein Dishevelled (Dvl) to the receptor complex via direct binding and subsequently multimerizes and induces formation of LRP-associated Wnt signalsomes (Bilic et al., 2007). The intra cellular region of LRP6 is phosphorylated by GSK3 $\beta$ or CK1 (casein kinase 1). The phosphorylated LRP6 and activated Dvl recruit Axin to the cell membrane, resulting in the dissociation of $\beta$-catenin from the destruction complex (Schwarz-Romond et al., 2007). Therefore $\beta$-catenin accumulates in the cytoplasm and enters into the nucleus to bind to TCF (T-cell factor) / LEF (lymphoid enhancer factor), thereby activating the Wnt transcriptional process (Fig. 1.7 B) (reviews of MacDonald, Tamai and He, 2009 and Tolwinski and Wieschaus, 2004). 


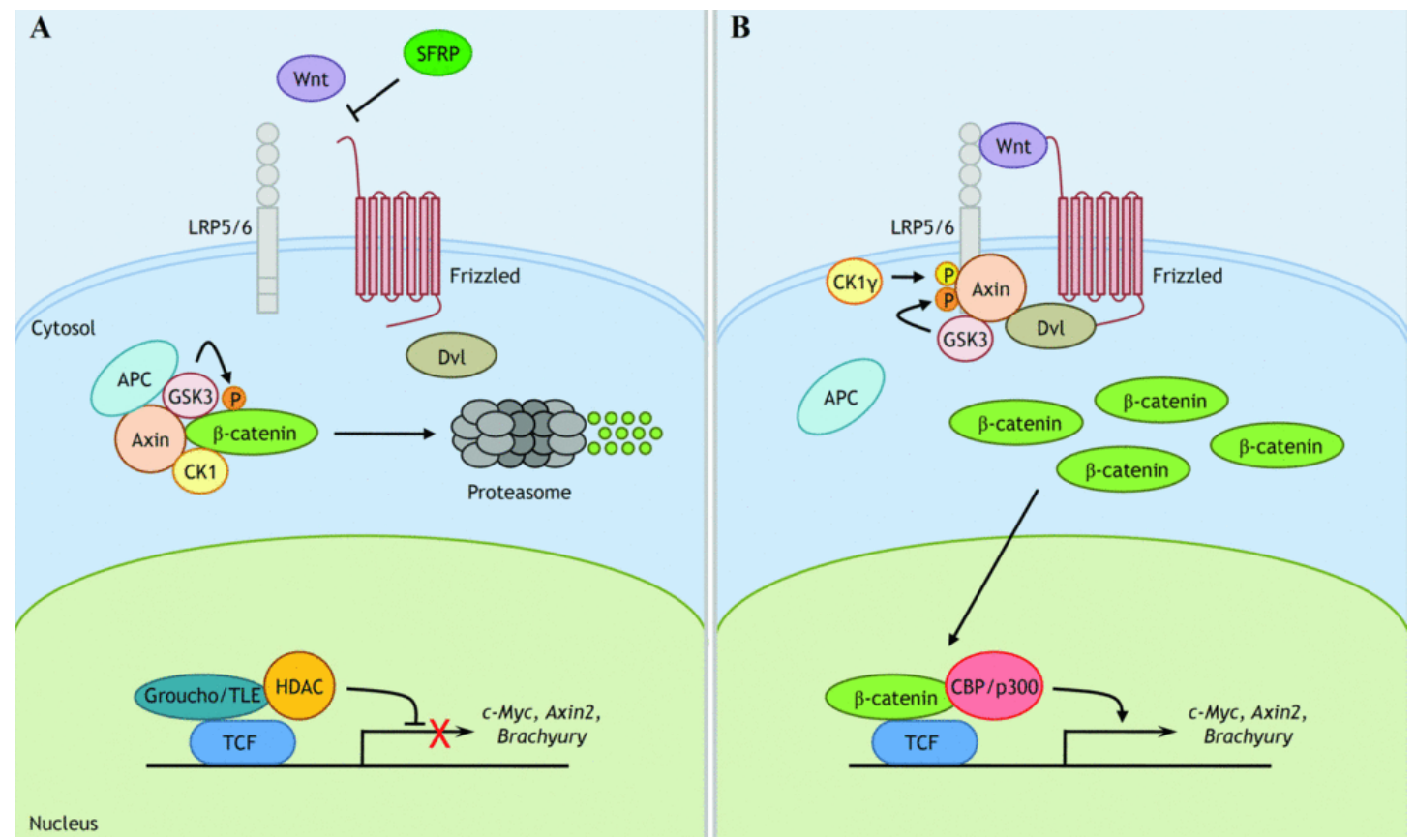

Fig. 1.7: Wnt/ $\beta$-catenin signalling pathway. (A), in the absence of Wnt ligands. $\beta$-catenin is phosphorylated by the destruction complex (APC, Axin, and GSK3 $\beta$ ) and subsequently marked for degradation by the ubiquitin-E3 ligase $\beta$-TrCP. (B), in the presence of Wnt ligands. Wnt binds to Fz and LRP6, then LRP5/6 is phosphorylated by GSK3 $\beta$ and CK1 $\gamma$, after which Axin is recruited to the phospho-LRP5/6 tail. These processes induce dissociation of the $\beta$-catenin destruction complex and subsequent accumulation of cytosolic $\beta$-catenin. Accumulated $\beta$-catenin enters into the nucleus and interacts with TCF / LEF1 for activation of Wnt-responsive genes. SFRP, secreted Fz-related protein (review of Kim, Kim and Jho, 2013).

\subsection{1 $\beta$-catenin protein}

$\beta$-catenin (Armadillo in Drosophila) is a member of the Armadillo family of proteins, which have multiple copies of the so-called armadillo repeat domain ( $\sim 2$ amino acids) composed of three alpha helices (Peifer et al., 1994). In the canonical Wnt pathway, as the key protein $\beta$-catenin is responsible for transduction of the signal to the nucleus and thereby the Wnt transcriptional process is activated (Fig. 1.8). Without a Wnt signal, the levels of cytoplasmic free $\beta$-catenin are kept low and the majority of $\beta$-catenin is located at the cytoplasmic side of the membrane as a component of 
cadherin-based cell-cell connections. $\beta$-catenin, together with $\alpha$-catenin and $\gamma$-catenin / plakoglobin, link E-cadherin, the key molecule of the $\mathrm{Ca}^{2+}$-dependent cell adhesion, to cytoskeletal structures (Ozawa et al., 1989). Besides these signaling and structural functions, $\beta$-catenin was found surprisingly in the centrosome and contributes to centrosomal cohesion and separation at the onset of mitotic spindle formation (Fig. 1.8) (Huang et al., 2007).

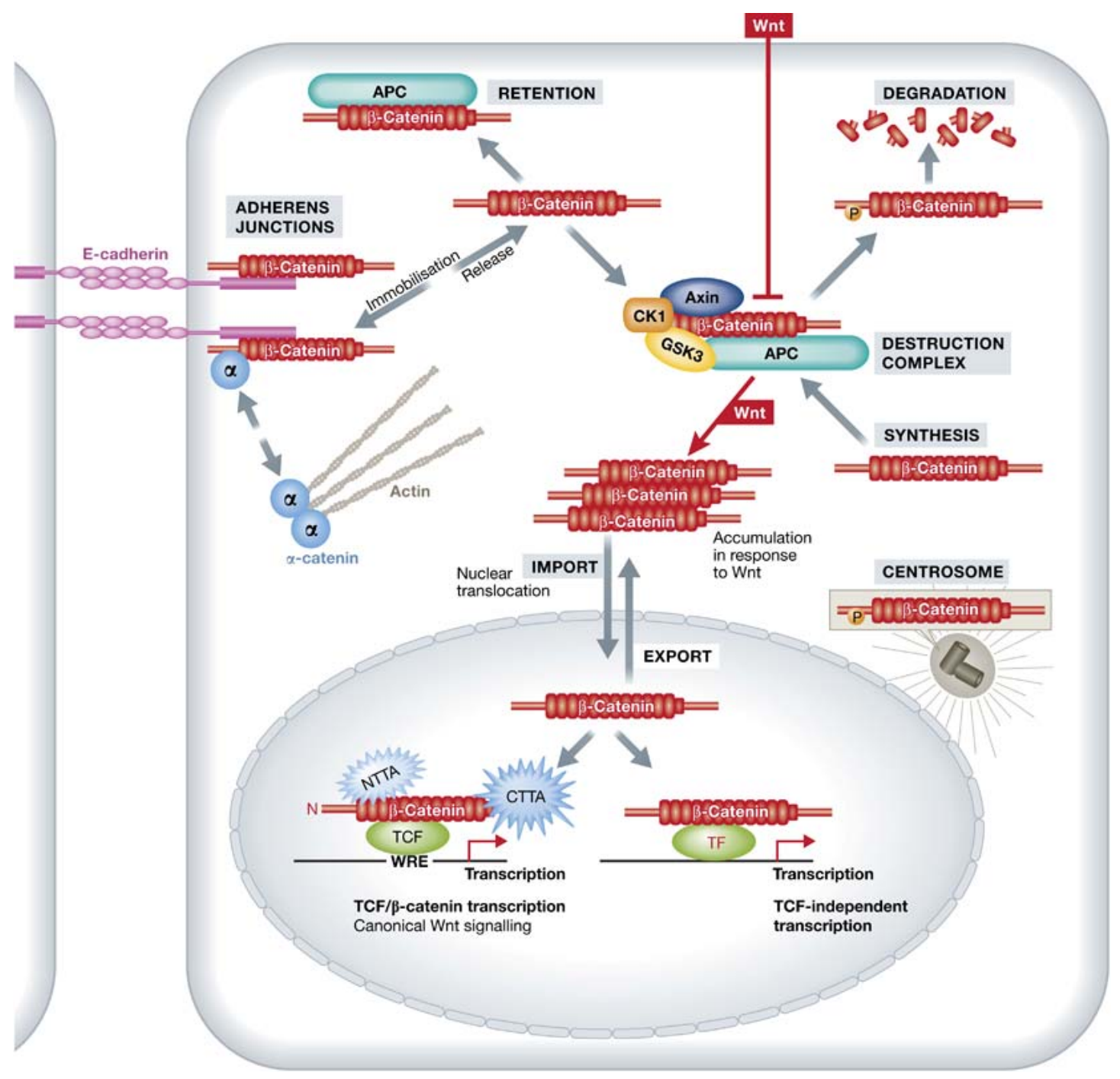

Fig. 1.8: The faces of $\beta$-catenin within the cell. So far, $\beta$-catenin has three main functions in the cell: (1) $\beta$-catenin is responsible for transduction of the signal to the nucleus in the case of Wnt-on and thereby the Wnt transcriptional process is activated whereas without Wnt signal the most cytoplasmic $\beta$-catenin is phosphorylated by destruction complex and subsequently marked for 
degradation by the ubiquitin-E3 ligase $\beta$-TrCP. (2) $\beta$-catenin is located at the cytoplasmic side of the membrane and links E-cadherin to cytoskeletal structures. (3) $\beta$-catenin contributes to centrosomal cohesion and separation at the onset of mitotic spindle formation (review of Valenta, Hausmann and Basler, 2012).

\subsection{Goals of the thesis}

The outer dense fibers (ODFs) are prominent sperm tail-specific cytoskeletal structures. They seem to be important for the stability and the elastic recoil of the sperm tail as well as for support of the flagellar beat (Baltz et al., 1990; Lindemann, 1996). Impaired development of the outer dense fibers has been described as a major cause of tail abnormalities in infertile men (Haidl et al., 1991) indicating an important function in sperm motility and/or morphology. The ODFs are composed of at least 14 polypeptides of which only a few have been identified. So far, the functions of the most proteins of the ODFs are unknown. In this study, I was focusing on functional analyses of two components of ODFs: ODF1 and ODF2. ODF1 specifically expressed in spermatids, ODF2 ubiquitously expressed.

1. To study ODF1 function in formation of the sperm tail cytoskeleton and its impact for stability and motility Odf1-knock out mice were generated, subsequently, the phenotypes of these mice were analysed.

2. To find out the function of ODF2 at the appendages of the mother centriole in somatic cells the influence of ODF2 on canonical Wnt pathway key protein $\beta$-catenin was analysed. 


\section{Material and methods}

\subsection{Material}

\subsubsection{Biological material}

\subsubsection{Bacterial stains}

Eschericia coli strain DH5 $\alpha$ was used for subcloning and keeping of plasmid DNA.

\subsubsection{Cell lines}

For analysis the following cell lines were used:

ES: mouse embryonic stem cells

SSC: mouse spermatogonial stem cells

F9: mouse teratocarcinoma cells

NIH3T3: mouse embryonic fibroblast cell line

HEK293: human embryonic kidney cell line

MCF7: human breast adenocarcinoma cell line

MDA-MB-231: human breast adenocarcinoma cell line

\subsubsection{Vectors}

\begin{tabular}{|l|l|l|l|}
\hline \multicolumn{1}{|c|}{ Vector } & \multicolumn{1}{c|}{ Resource } & \multicolumn{1}{c|}{ Generated constructs } & \multicolumn{1}{c|}{ Reference } \\
\hline pBluescript & Stratagene & Odfl & Schweizer \\
\hline pPNT-M1 & $\begin{array}{l}\text { Tybulewicz et al., } \\
1991\end{array}$ & Odf1 & Schweizer \\
\hline pGEMT & Promega & Odf1 & Schweizer \\
\hline pECFP-N1 & Clontech & Odf1 & Hoyer-Fender \\
\hline $\begin{array}{l}\text { pcDNA3.1/myc-His } \\
(-) \text { C }\end{array}$ & $\begin{array}{l}\text { Invitrogen } \\
\text { Life technologies }\end{array}$ & Spag4 & Hoyer-Fender \\
\hline $\begin{array}{l}\text { pcDNA3.1HA/myc- } \\
\text { His (-) C }\end{array}$ & $\begin{array}{l}\text { Invitrogen } \\
\text { LM } \\
\text { Life technologies }\end{array}$ & $\begin{array}{l}\text { Spag4; Spag4-N; Spag4-C; } \\
\text { EYFP-Spag4 }\end{array}$ & Hoyer-Fender \\
\hline
\end{tabular}




\begin{tabular}{|c|c|c|c|}
\hline pEGFP-N1 & Clontech & $\begin{array}{l}\text { 13.8NC-GFP; } 13.8 N C \triangle G F P ; \\
13.8 ; \text { N-terminal; human Odf } 2 ; \\
\text { cenexin; cenexin insertion; } \\
\text { ODF2NC; } \quad \text { ODF } 2 N 2 C ; \\
\text { ODF2NC1; } \\
\text { pEGFP-1; ninein }\end{array}$ & Hoyer-Fender \\
\hline phRL-SV40 & Promega & 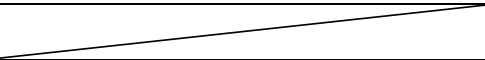 & - \\
\hline pGL3 & Promega & Fopflash (OF) & Vogelstein \\
\hline pGL3 & Promega & Topflash (OT) & Vogelstein \\
\hline pCI-neo & Promega & $\beta$-catenin & Vogelstein \\
\hline pIRES-EGFP & Clontech & shODF2 & Haupt \\
\hline pCS2 & $\begin{array}{l}\text { Invitrogen }^{\mathrm{TM}} \\
\text { Life technologies }\end{array}$ & Odf2, Xwnt-8, GFP & Henningsfeld \\
\hline pQM-NTag/B & Abcam & $+2=$ & 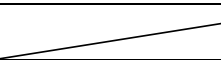 \\
\hline $\begin{array}{l}\text { FPCAV2 (modified } \\
\text { from pQM-NTag/B) }\end{array}$ & Hoyer-Fender & $\alpha$-tubulin; $\gamma$-tubulin; $\varepsilon$-tubulin & Hoyer-Fender \\
\hline
\end{tabular}

Tab. 2.1: Vectors.

\subsubsection{Oligonucleotides}

\begin{tabular}{|l|l|l|l|}
\hline \multicolumn{1}{|c|}{ Name } & Target gene & Species & \multicolumn{1}{c|}{ Sequence } \\
\hline Odf1SPst & Odf1 & mouse & CTGCAGCTCAGAGTCTTGTCTGTG \\
\hline Odf1SHindIII & Odf1 & mouse & AAGCTTAGACTGTTCTCCACAG \\
\hline Odf1-I & Odf1 & mouse & ATCAACTCTGCCTGAGAC \\
\hline Neomy & Odf1 & mouse & CCTTCTATCGCCTCCTTGACG \\
\hline Odf1-5'ko & Odf1 & mouse & AGGAAGAAGGGACTAGAG \\
\hline Odf1-N & Odf1 & mouse & GAGCTCAAGCTTTGGCCGCACTGAGTTGTC \\
\hline MSYCP35 Kpn1 & SYCP3 & mouse & GACGGTACCATGCTTCGAGGGTGTGGG \\
\hline MSYCP3r & SYCP3 & mouse & TTGACACAATCGTGGAGAGAA \\
\hline Stra8 for & Stra8 & mouse & TCACAGCCTCAAAGTGGCAGG \\
\hline Stra8 rev & Stra8 & mouse & GCAACAGAGTGGAGGAGGAGT \\
\hline Hanp1-H1T2-for & HANP1/H1T2 & mouse & GCTGGCTACTTCAGGGTCT \\
\hline Hanp1-H1T2-rev & HANP1/H1T2 & mouse & TGTATGCTGGGAGCGTTG \\
\hline MMISf & IIS & mouse & TTGGTGCTAACCGTGGACTT \\
\hline MMISr & IIS & mouse & GCAGAGCACGAACCAAGCGA \\
\hline mIns13f & Ins 13 & mouse & TACTGATGCTCCTGGCTCTGG \\
\hline mIns13r & Ins 13 & mouse & TTAGACTGTTTGGGACACAGG \\
\hline 138NCEcoRI & Odf2 & mouse & GGAATTCATGTCCGCCTCATCCTCAGGC \\
\hline DH1rev & Odf2 & mouse & CAGCTTCCCGATGGTATCCTTCAAG \\
\hline Odf1-C & Odf1 & mouse & CCGCGGTACCCAAGATCATCTTCCTACA \\
\hline SPAG4 C KpnI & Spag4 & mouse & GGTACCGATGGGGTCCCCCTGTGAC \\
\hline
\end{tabular}




\begin{tabular}{|l|l|l|l|}
\hline Spag4 650 for & Spag4 & mouse & TGCAGGGAAATCTGCTCC \\
\hline SPAG4 N XbaI & Spag4 & mouse & TCTAGAATGCGGCGGAGCCCCCGC \\
\hline SPAG4N2XbaI & Spag4 & mouse & TCTAGAGAACCTACGGAGATGCTGAC \\
\hline SPAG4C2KpnI & Spag4 & mouse & GGTACCTTAGAGTCAGCATCTCCGTAGGTTC \\
\hline EYFP-C XbaI & eYfp & mouse & TCTAGACTTGTACAGCTCGTCCATGC \\
\hline EYFP-N SpeI & eYfp & mouse & ACTAGTATGGTGAGCAAGGGCGAGG \\
\hline GAPDHfor & Gapdh & mouse & CACCACCAACTGCTTAGCC \\
\hline GAPDHrev & Gapdh & mouse & CGGATACATTGGGGGTAGG \\
\hline hHPRT1_2f_390 & $H P R T$ & human & TTTGCTGACCTGCTGGAT \\
\hline hHPRT1_2r_631 & $H P R T$ & human & GGATTATACTGCCTGACCAAG \\
\hline hCmyc_2f_344 & $C-M y c$ & human & TTGCCGCATCCACGAAAC \\
\hline hCmyc_2r_615 & $C-M y c$ & human & GTCGAGGTCATAGTTCCTGTTGGT \\
\hline
\end{tabular}

Tab. 2.2: Oligonucleotides.

\subsubsection{Enzymes}

\begin{tabular}{|l|l|l|}
\hline \multicolumn{1}{|c|}{ Enzyme type } & \multicolumn{1}{c|}{ Enzyme } & \multicolumn{1}{c|}{ Resource } \\
\hline \multirow{4}{*}{ Restrication enzyme } & XbaI & \multirow{2}{*}{ Fermentas } \\
\cline { 2 - 2 } & EcoRI & \\
\cline { 2 - 2 } & NotI & \\
\cline { 2 - 2 } & XhoI & \\
\cline { 2 - 2 } & PstI & Bioline \\
\cline { 2 - 3 } & HindIII & Fermentas \\
\cline { 2 - 3 } & KpnI & Roche \\
\hline DNA-polymerase & Mango Taq DNA polymerase & Fermentas \\
\cline { 2 - 3 } & Taq polymerase & Sigma-Aldrich \\
\hline DNase & RQ1 DNase & Thermo Scientific \\
\hline DNA ligase & T4 DNA ligase & $\begin{array}{l}\text { Supplied by human genetic institute } \\
\text { (Göttingen) }\end{array}$ \\
\hline Proteinase kinase & Proteinase K & \\
\hline Protease inhibitor & protease inhibitor cocktail (100x) & \\
\hline Hyaluronidase & Hyaluronidase & \\
& & \\
\hline
\end{tabular}

Tab. 2.3: Enzymes. 


\subsubsection{Antibodies}

\subsubsection{Primary antibodies}

\begin{tabular}{|c|c|c|c|}
\hline Target protein & Antibody name & Host & Resource \\
\hline$\alpha$-tubulin & $\begin{array}{l}\text { anti- } \alpha \text {-tubulin mouse monoclonal } \\
\text { antibody (DM1A) }\end{array}$ & mouse & Calbiochem \\
\hline SPAG4 & anti-SPAG4 antibody (sc-85927) & rabbit & Santa Cruz Biotechnology \\
\hline ODF1 & Anti-ODF1 & rabbit & $\begin{array}{l}\text { The Abs were affinity purified } \\
\text { as described by Brohmann et } \\
\text { al. (1997) using an antiserum } \\
\text { against total ODF proteins and } \\
\text { bacterially expressed mouse } \\
\text { ODF1 fused to MBP (maltose } \\
\text { binding protein; } p O d f 1-M B P \text { ). }\end{array}$ \\
\hline GFP & Anti-GFP & rabbit & Invitrogen \\
\hline Myc-tag & Anti-myc polyclonal & rabbit & Millipore \\
\hline HA-tag & HA.C5 & mouse & Abcam \\
\hline$\gamma$-tubulin & GTU-88 & mouse & Sigma \\
\hline$\beta$-catenin & $\begin{array}{l}\text { Rabbit polyclonal to beta Catenin } \\
\text { (ab6302) }\end{array}$ & rabbit & Abcam \\
\hline Axin1 & Axin1 (C76H11) Rabbit mAb & rabbit & Cell Signaling Technology \\
\hline Axin2 & Axin2 (76G6) Rabbit mAb & rabbit & Cell Signaling Technology \\
\hline Phospho-Tau & p-Tau (Ser 404) & rabbit & Santa Cruz Biotechnology \\
\hline
\end{tabular}

Tab. 2.4: Primary antibodies.

\subsubsection{Secondary antibodies}

\begin{tabular}{|l|l|l|l|}
\hline \multicolumn{1}{|c|}{ Target protein } & \multicolumn{1}{c|}{ Antibody name } & \multicolumn{1}{c|}{ Host } & \multicolumn{1}{c|}{ Resource } \\
\hline \multirow{2}{*}{ mouse IgG } & Anti mouse-HRP 4759 & goat & Carl Roth \\
\cline { 2 - 4 } & MFP 590 & goat & Molecular Probes \\
\hline \multirow{2}{*}{ Rabbit IgG } & Anti rabbit IgG HRP & goat & Sigma Biosciences \\
\cline { 2 - 4 } & Alexa 488 & goat & Molecular Probes \\
\hline
\end{tabular}

Tab. 2.5: Secondary antibodies. 


\subsubsection{Culture media for $E$. coli cultures}

\begin{tabular}{|l|l|}
\hline \multicolumn{1}{|c|}{ Name } & \multicolumn{1}{c|}{ Ingredients } \\
\hline LB medium (Luria Bertani medium) & $10 \mathrm{~g}$ tryptone \\
& $5 \mathrm{~g}$ yeast extract \\
& $10 \mathrm{~g} \mathrm{NaCl}$ \\
& $\mathrm{pH} 7,5$ \\
& diluted in milliq $\mathrm{H}_{2} \mathrm{O}$ \\
\hline LB agar plate & $1,5 \%(\mathrm{w} / \mathrm{v})$ agar in $\mathrm{LB}$ medium \\
\hline Ampicillin medium & $100 \mu \mathrm{g} / \mathrm{ml}$ ampicillin in LB medium \\
\hline Kanamycin medium & $50 \mu \mathrm{g} / \mathrm{ml}$ kanamycin in LB medium \\
\hline
\end{tabular}

Tab. 2.6: Culture media for $E$. coli cultures.

\subsubsection{Culture media for eukaryotic cell culture}

\begin{tabular}{|c|c|}
\hline Cell line & Ingredients \\
\hline $\begin{array}{l}\text { NIH3T3 } \\
\text { HEK293 }\end{array}$ & $\begin{array}{l}\text { Gibco DMEM + Glutamax-I 1x } 500 \mathrm{ml} \\
\text { (contains } 4,5 \mathrm{~g} / 1 \text { D-glucose and sodium pyruvate) } \\
\text { penicillin-streptomycin } 1 \% \\
\text { FBS } 10 \%\end{array}$ \\
\hline MCF7 & $\begin{array}{l}\text { Gibco DMEM + Glutamax-I 1x } 500 \mathrm{ml} \\
\text { (contains } 4,5 \mathrm{~g} / 1 \text { D-glucose and sodium pyruvate) } \\
\text { penicillin-streptomycin } 1 \% \\
\text { FBS } 10 \% \\
\text { NEAA } 500 \mu \mathrm{l} \\
\text { insulin } 2 \mathrm{mg}\end{array}$ \\
\hline MDA-MB-231 & $\begin{array}{l}\text { RPMI medium } 1640+\text { Glutamax-I 1x } 500 \mathrm{ml} \\
25 \mathrm{mM} \text { HEPES } \\
\text { Penicillin-Streptomycin } 1 \% \\
\text { FBS } 10 \%\end{array}$ \\
\hline
\end{tabular}

Tab. 2.7: Culture media for eukaryotic cell culture.

Besides insulin all media and supplements were ordered from Gibco Life Technologies. Insulin was ordered from Sigma-Aldrich.

Freezing medium: Complete medium with additional 10\% FBS and 10\% DMSO (Sigma-Aldrich).

Serum starvation medium is complete medium but only with $0.5 \%$ FBS. 


\subsubsection{Buffers and solutions}

\begin{tabular}{|c|c|}
\hline Name & Ingredients \\
\hline Acid-ethanol & $250 \mu \mathrm{l}$ of $37 \% \mathrm{HCl}$ in $70 \mathrm{ml}$ of $70 \%$ ethanol \\
\hline Ammoniac- $\mathrm{H}_{2} \mathrm{O}$ & $100 \mu \mathrm{l}$ of $30-33 \%$ ammoniac in $80 \mathrm{ml} \mathrm{H}_{2} \mathrm{O}$ \\
\hline Bouin's solution & $\begin{array}{l}15 \mathrm{v} \text { of } 1.2 \% \text { picric acid } \\
5 \mathrm{v} \text { of } 37 \% \text { PFA } \\
1 \mathrm{v} \text { glacial acetic acid }\end{array}$ \\
\hline Buffer A & $\begin{array}{l}15 \mathrm{mM} \mathrm{MgSO}_{4} \\
0.1 \mathrm{mM} \text { EDTA } \\
25 \mathrm{mM} \text { DTT } \\
1 \mathrm{mM} \text { ATP } \\
200 \mu \mathrm{M} \text { coenzyme A } \\
200 \mu \mathrm{M} \text { D-luciferin } \\
200 \mathrm{mM} \text { Tris } \\
\text { final pH } 8.0 \\
\end{array}$ \\
\hline Buffer B & $\begin{array}{l}10 \mathrm{mM} \text { sodium acetate } \\
15 \mathrm{mM} \text { EDTA } \\
500 \mathrm{mM} \mathrm{NaCl} \\
50 \mu \mathrm{M} \mathrm{APMBT} \\
4 \mu \mathrm{M} \text { benzyl-coelenterazine } \\
500 \mathrm{mM} \mathrm{Na}_{2} \mathrm{SO}_{4} \\
25 \mathrm{mM} \mathrm{Na}_{4} \mathrm{PPi} \\
\text { final } \mathrm{pH} 5.0\end{array}$ \\
\hline Denaturation buffer $(8 \mathrm{x})$ & $\begin{array}{l}250 \mathrm{mM} \text { Tris- } \mathrm{HCl}(\mathrm{pH} 6.8) \\
8 \%(\mathrm{w} / \mathrm{v}) \text { SDS } \\
40 \%(\mathrm{w} / \mathrm{v}) 2-\beta-M e r c a p t o e t h a n o l \\
0.004 \%(\mathrm{w} / \mathrm{v}) \text { bromophenol blue } \\
40 \%(\mathrm{w} / \mathrm{v}) \text { glycerol }\end{array}$ \\
\hline DNA loading buffer $(6 x)$ & $\begin{array}{l}0.09 \%(\mathrm{w} / \mathrm{v}) \text { xylene cyanol FF } \\
60 \mathrm{mM} \text { EDTA } \\
0.09 \%(\mathrm{w} / \mathrm{v}) \text { bromophenol blue } \\
60 \%(\mathrm{w} / \mathrm{v}) \text { glycine }\end{array}$ \\
\hline Ethidium bromide & $\begin{array}{l}10 \mathrm{mg} / \mathrm{ml} \text { ethidium bromide } \\
\text { Working solution } 5 \mu \mathrm{g} / \mathrm{ml}\end{array}$ \\
\hline IVF Cook medium & Supplied by human genetic institute (Göttingen) \\
\hline KHM buffer & $\begin{array}{l}110 \mathrm{mM} \text { KOAc } \\
20 \mathrm{mM} \text { Hepes (pH 7.4) } \\
2 \mathrm{mM} \mathrm{MgCl}_{2}\end{array}$ \\
\hline $\begin{array}{l}\text { Laemmli electrophoreses buffer } \\
(10 \mathrm{x})\end{array}$ & $\begin{array}{l}0.25 \mathrm{M} \text { Tris } \\
2 \mathrm{M}(\mathrm{w} / \mathrm{v}) \text { glycine } \\
1 \%(\mathrm{w} / \mathrm{v}) \text { SDS }\end{array}$ \\
\hline Lysis buffer for Co-IP & $150 \mathrm{mM} \mathrm{NaCl}$ \\
\hline
\end{tabular}




\begin{tabular}{|c|c|}
\hline & $\begin{array}{l}2 \text { mM EDTA } \\
1 \% \text { Triton X-100 } \\
1 \mathrm{x} \text { of protease inhibitor cocktail }(100 \mathrm{x}) \\
\text { in PBS }(\mathrm{pH} 7.2)\end{array}$ \\
\hline $\begin{array}{l}\text { Lysis buffer for in situ proteinase } \mathrm{K} \\
\text { digestion }\end{array}$ & $\begin{array}{l}0.4 \%(\mathrm{w} / \mathrm{v}) \mathrm{SDS} \\
2 \% \text { Triton X-100 } \\
400 \mathrm{mM} \mathrm{NaCl} \\
50 \mathrm{mM} \text { Tris- } \mathrm{HCl}(\mathrm{pH} 7.4) \\
1 \mathrm{mM} \text { DTT } \\
1 \mathrm{x} \text { of protease inhibitor cocktail }(100 \mathrm{x})\end{array}$ \\
\hline Lysis buffer for reporter gene assay & $\begin{array}{l}0.25 \% \text { Triton X-100 } \\
\text { in } 150 \mathrm{mM} \text { Hepes }(\mathrm{pH} 8.0)\end{array}$ \\
\hline $5 \%$ Milk buffer & $5 \%(\mathrm{w} / \mathrm{v})$ skimmed milk powder (slightly soluble) in TBST \\
\hline MBS & $\begin{array}{l}88 \mathrm{mM} \mathrm{NaCl} \\
1 \mathrm{mM} \mathrm{KCl} \\
2.4 \mathrm{mM} \mathrm{NaHCO}_{3} \\
15 \mathrm{mM} \mathrm{Hepes}(\mathrm{pH} 7.6) \\
0.3 \mathrm{mM} \mathrm{Ca}\left(\mathrm{NO}_{3}\right)_{2} \\
0.41 \mathrm{mM} \mathrm{CaCl}_{2} \\
0.82 \mathrm{mM} \mathrm{MgSO} \\
50 \mu \mathrm{g} / \mathrm{ml} \text { gentamycin } \\
\end{array}$ \\
\hline PBT & $\begin{array}{l}1 \%(\mathrm{w} / \mathrm{v}) \mathrm{BSA} \\
0.5 \% \text { Tween } 20 \\
\text { in PBS }(\mathrm{pH} 7.5) \\
\end{array}$ \\
\hline PBS & $\begin{array}{l}145 \mathrm{mM} \mathrm{NaCl} \\
7 \mathrm{mM} \mathrm{Na}_{2} \mathrm{HPO}_{4} \\
3 \mathrm{mM} \mathrm{NaH}_{2} \mathrm{PO}_{4} \\
\end{array}$ \\
\hline $\operatorname{SSC}(20 x)$ & $\begin{array}{l}3 \mathrm{M} \mathrm{NaCl} \\
0.3 \mathrm{M} \text { trisodium citrate } \\
\text { final } \mathrm{pH} 7.0\end{array}$ \\
\hline SSPE (20x) & $\begin{array}{l}.6 \mathrm{M} \mathrm{NaCl} \\
0.2 \mathrm{M} \mathrm{NaH}_{2} \mathrm{PO}_{4} \\
0.02 \mathrm{M} \mathrm{Na}_{2} \text { EDTA } \\
\text { final pH } 7.7\end{array}$ \\
\hline Stripping solution & $\begin{array}{l}1.5 \%(\mathrm{w} / \mathrm{v}) \text { glycine } \\
0.1 \%(\mathrm{w} / \mathrm{v}) \text { SDS } \\
1 \% \text { Tween } 20 \\
\text { Final } \mathrm{pH} 2.2 \\
\end{array}$ \\
\hline TAE buffer (50x) & $\begin{array}{l}242 \mathrm{~g} / 1 \text { Tris } \\
57.1 \mathrm{ml} / 1 \text { glacial acetic acid } \\
100 \mathrm{ml} \text { of } 0.5 \mathrm{M} \text { EDTA } \\
\text { final } \mathrm{pH} 8.0\end{array}$ \\
\hline
\end{tabular}




\begin{tabular}{|l|l|}
\hline TBST & $20 \mathrm{mM}$ Tris- $\mathrm{HCl}(\mathrm{pH} 7.5)$ \\
& $300 \mathrm{mM} \mathrm{NaCl}$ \\
& $0.2 \%$ triton $\mathrm{X}-100$ \\
\hline Transfer buffer & $1 \mathrm{M}$ Tris \\
& $20 \mathrm{M}$ glycine \\
& $0.4 \mathrm{mM} \mathrm{MgCl}$ \\
& $0.04 \%(\mathrm{w} / \mathrm{v}) \mathrm{SDS}$ \\
& $8 \%$ methanol \\
\hline Tyrode's medium & $8 \mathrm{~g} / 1 \mathrm{NaCl}$ \\
& $200 \mathrm{mg} / 1 \mathrm{KCl}$ \\
& $200 \mathrm{mg} / 1 \mathrm{CaCl}_{2}$ \\
& $100 \mathrm{mg} / 1 \mathrm{MgCl}_{2}$ \\
& $50 \mathrm{mg} / 1 \mathrm{NaH}_{2} \mathrm{PO}_{4}$ \\
& $1 \mathrm{~g} / 1 \mathrm{NaHCO}$ \\
& $1 \mathrm{~g} / 1 \mathrm{glucose}$ \\
& final pH 6.5 \\
\hline
\end{tabular}

Tab. 2.8: Buffers and solutions. If no other indications, solutions and buffers were not high concentrated and prepared in milliq $\mathrm{H}_{2} \mathrm{O}$.

\subsubsection{Chemicals}

\begin{tabular}{|l|l|}
\hline \multicolumn{1}{|c|}{ Name } & \multicolumn{1}{c|}{ Producer } \\
\hline Agarose & Carl Roth \\
\hline Ammoniac (30-33\%) & Roth \\
\hline Ampicillin & AppliChem \\
\hline APMBT & AppliChem \\
\hline APS & Sigma-Aldrich \\
\hline ATP & Bioline \\
\hline Benzyl-coelenterazine & Synchem \\
\hline Bromophenol blue & Sigma-Aldrich \\
\hline BSA & AppliChem \\
\hline CaCl 2 & Merck \\
\hline Calcium ionophore A23187 & Sigma-Aldrich \\
\hline Ca(NO $)_{2}$ & Sigma-Aldrich \\
\hline Coenzyme A & AppliChem \\
\hline Coomassie brilliant blue R250 & Sigma-Aldrich \\
\hline DAPI & Sigma-Aldrich \\
\hline 50x Denhardt's solution & Invitrogen ${ }^{\text {TM }}$ of Life Technologies \\
\hline D-luciferin & Carl Roth \\
\hline DTT & Sigma-Aldrich \\
\hline EDTA & Carl Roth \\
\hline Eosin G-solution $0.5 \%$ diluted & Carl Roth \\
\hline &
\end{tabular}




\begin{tabular}{|c|c|}
\hline Ethanol & VWR Chemicals \\
\hline Ethidium bromide & Carl Roth \\
\hline Ficoll & Supplied by Henningfeld's group \\
\hline G418 & PAA Laboratories GmbH \\
\hline Gentamycin & Supplied by Henningfeld's group \\
\hline Glacial acetic acid & Carl Roth \\
\hline Glucose & Supplied by human genetic institute (Göttingen) \\
\hline Glycerol & Roth \\
\hline Glycine & Roth \\
\hline $\mathrm{HCG}$ & Supplied by human genetic institute (Göttingen) \\
\hline $\mathrm{HCl}(37 \%)$ & Roth \\
\hline Hematoxylin solution (Gill II) & Carl Roth \\
\hline HEPES & Roth \\
\hline Kanamycine & Sigma-Aldrich \\
\hline $\mathrm{KCl}$ & Merck \\
\hline KOAc & Roth \\
\hline 2- $\beta$-Mercaptoethanol & Sigma-Aldrich \\
\hline Methanol & VWR Chemicals \\
\hline $\mathrm{MgCl}_{2}$ & AppliChem \\
\hline $\mathrm{MgSO}_{4}$ & AppliChem \\
\hline $\mathrm{NaCl}$ & AppliChem \\
\hline $\mathrm{Na}_{2}$ EDTA & Roth \\
\hline $\mathrm{NaHCO}_{3}$ & Merck \\
\hline $\mathrm{NaH}_{2} \mathrm{PO}_{4}$ & Merck \\
\hline $\mathrm{Na}_{2} \mathrm{HPO}_{4}$ & Roth \\
\hline $\mathrm{Na}_{4} \mathrm{PPi}$ & AppliChem \\
\hline $\mathrm{Na}_{2} \mathrm{SO}_{4}$ & Merck \\
\hline Nocodazol & Sigma-Aldrich \\
\hline Nonidet P40 & Fluka Chemie AG \\
\hline Paraffin & Merck \\
\hline Picric acid & Sigma-Aldrich \\
\hline PFA & Sigma-Aldrich \\
\hline PL-FITC & PL biochemicals \\
\hline PMSF & Sigma-Aldrich \\
\hline PMSG & Supplied by human genetic institute (Göttingen) \\
\hline $\begin{array}{l}\text { Renaissance Enhanced Luminol Western } \\
\text { Blot Chemiluminescence Reagent Plus }\end{array}$ & NEN Life Science Products \\
\hline RNA Secure ${ }^{\mathrm{TM}}$ Resuspension solution & Ambion \\
\hline Rotiphorese $^{\circledR}$ gel $40(29: 1)$ & Roth \\
\hline Salmon sperm DNA & Sigma-Aldrich \\
\hline SDS & Roth \\
\hline skimmed milk powder (slightly soluble) & SUCOFIN $^{\circledR}$ \\
\hline Sodium acetat & AppliChem \\
\hline
\end{tabular}




\begin{tabular}{|l|l|}
\hline TEMED & Merck \\
\hline Tris & Roth \\
\hline Trisodium citrate & Roth \\
\hline Triton X-100 & Sigma-Aldrich \\
\hline Tween 20 & Merck \\
\hline Xylene cyanol FF & Sigma-Aldrich \\
\hline Xylol & Roth \\
\hline
\end{tabular}

Tab. 2.9: Chenicals.

\subsubsection{Kits}

\begin{tabular}{|l|l|}
\hline \multicolumn{1}{|c|}{ Name } & \multicolumn{1}{c|}{ Producer } \\
\hline EndoFree $^{\circledR}$ Plasmid Purification & Qiagen GmbH \\
\hline ExoIII/S1 deletion kit & MBI Fermentas \\
\hline in vitro fertilization medium & Medi-Cult \\
\hline iQ $^{\mathrm{TM}}$ SYBR $^{\circledR}$ Green Supermix & Bio-Rad \\
\hline Mini prep kit NucleoSpin ${ }^{\circledR}$ plasmid & Macherey-Nagel GmbH\&CoKG \\
\hline peqGOLD RNAPure & PEQLAB \\
\hline peqGOLD TriFast reagent & PEQLAB \\
\hline protein G agarose & Thermo Scientific \\
\hline QIAEX ${ }^{\circledR}$ II Gel Extraction kit (150) & Qiagen GmbH \\
\hline RevertAid H Minus First Strand cDNA synthesis kit & Fermentas \\
\hline Transfectin & Bio-Rad \\
\hline Viagen DirectPCR-Tail & Viagen Biotech \\
\hline X-tremeGENE HP DNA Transfection Reagent & Roche \\
\hline
\end{tabular}

Tab. 2.10: Kits. 


\subsection{Methods}

\subsubsection{General methods}

\subsubsection{Microbiological methods}

\subsection{Culture of $E$. coli}

E. coli were cultured over night at $37{ }^{\circ} \mathrm{C}$ at $220 \mathrm{rpm}$.

\subsection{Storage of E.coli cultures}

$200 \mu \mathrm{l}$ glycerine was added to $800 \mu \mathrm{l}$ over night bacterial culture and gently mixed. The mixtures were stored at $-80{ }^{\circ} \mathrm{C}$.

\subsection{Production of competent bacteria}

$1 \mathrm{ml}$ over night bacterial culture was added to $100 \mathrm{ml} \mathrm{LB}$ medium and incubated at 37 ${ }^{\circ} \mathrm{C}$ until $\mathrm{OD}_{600}$ up to 0.35 to 0.4 . After incubation the bacterial culture was centrifuged for $20 \mathrm{~min}$ at $4{ }^{\circ} \mathrm{C}$ at $3000 \mathrm{x}$ g. The pellet was resuspended with $500 \mu \mathrm{l}$ precooled 50 $\mathrm{mM} \mathrm{CaCl}$. Subsequently the suspension was added into $20 \mathrm{ml} 50 \mathrm{mM} \mathrm{CaCl}$ and vibrated for $30 \mathrm{~min}$ at $4{ }^{\circ} \mathrm{C}$. The suspension was then centrifuged for $20 \mathrm{~min}$ at $4{ }^{\circ} \mathrm{C}$ at $3000 \mathrm{x}$ g. The pellet was repeatedly resuspended with $500 \mu \mathrm{l}$ precooled $50 \mathrm{mM} \mathrm{CaCl}_{2}$ and added into $5 \mathrm{ml} 50 \mathrm{mM} \mathrm{CaCl}{ }_{2} .200 \mu \mathrm{l}$ of the solution was aliquoted into Eppendorf reaction tube, immediately frozen in liquid nitrogen and stored at $-80{ }^{\circ} \mathrm{C}$.

\subsection{Transformation}

Transformation via heat shock into competent E. coli strains (DH5 $\alpha$ ) was performed as described by D. Hanahan (Hanahan, 1983). 
Bacteria cells were defrosted on ice and $50 \mu \mathrm{l}$ of cells was added to the DNA. The cells-DNA-mixture was incubated for $30 \mathrm{~min}$ on ice and then heated for $90 \mathrm{sec}$ at 42 ${ }^{\circ} \mathrm{C}$. After heat shock the cells were cooled for 2 min on ice. $300 \mu \mathrm{LB}$ medium without antibiotic was added to the cells and incubated for $1 \mathrm{~h}$ at $37^{\circ} \mathrm{C}$. The solution was added onto LB agar plate containing selective antibiotics and incubated over night at $37^{\circ} \mathrm{C}$.

\subsubsection{Molecular biological methods}

\subsection{DNA preparation}

Small amount of plasmid DNA was prepared with mini prep kit NucleoSpin ${ }^{\circledR}$ plasmid as indicated in the provided instruction manual. DNA for transfection was prepared with EndoFree ${ }^{\circledR}$ Plasmid Purification as indicated in the provided instruction manual. These protocols are based on the alkaline lysis from H.C. Birnboim and J. Doly (Birboim and Doly, 1979).

\subsection{DNA purification}

DNA was isolated from agarose gel and purified with QIAEX $^{\circledR}$ II Gel Extraction kit as indicated in the provided instruction manual.

\subsection{Measurement of DNA and RNA concentrations}

$1 \mu \mathrm{l}$ of the nucleic acid mixture was measured by $\mathrm{OD}_{260}$ with a photospectrometer (Nanodrop, PeqLab). The dilute reagent was used as blank reference. 


\subsection{Enzymatic modification of DNA by restriction enzymes}

$1 \mu \mathrm{g}$ DNA was digested by 1-1.5 U restriction enzyme in provided buffer. The enzyme volume should not be larger than $10 \%$ of total reaction volume.

\subsection{Ligation}

Ligations were performed with a 3:1 molar ratio insert/vector. $100 \mathrm{ng}$ of the vector was mixed with the insert DNA fragment. $1 \mu \mathrm{T}$ T4 DNA ligase with provided ligase buffer was added to the DNA mixture. Ligation was performed for $2 \mathrm{~h}$ at room temperature or over night at $4{ }^{\circ} \mathrm{C}$.

\subsubsection{Protein biochemical methods}

\subsection{Protein extraction and denaturation}

Mammalian cells were trypsinized and centrifuged for $3 \mathrm{~min}$ at $1000 \mathrm{x}$ g. The cells were washed with PBS and $1 \times 10^{6}$ cells resuspended in $32 \mu \mathrm{PBS} .4 \mu 18 \mathrm{x}$ denaturation buffer was added to the suspension. The mixture was boiled for $10 \mathrm{~min}$ at $95^{\circ} \mathrm{C}$.

\subsection{SDS-PAGE}

Proteins were separated with sodium dodecyl sulfate polyacrylamide gel electrophoresis (SDS-PAGE) (Laemmli, 1970). After denaturation of proteins the samples were loaded to the gel and separated in stacking gel in a SDS gel electrophoresis system (Bio-Rad) for $30 \mathrm{~min}$ at $100 \mathrm{~V}$. In resolving gel the samples ran for $1 \mathrm{~h}$ at $150 \mathrm{~V}$. 


\subsection{Transfer of proteins to Hybond ECL membrane}

For antibody detection of specific proteins the proteins were transferred onto a Hybond ECL membrane (Amersham Biosiences) (Towbin et al., 1979). The membrane was activated by methanol of transfer buffer. The gel together with membrane was clamped into Whatman papers and sponges. The blotting sandwich was placed in the blot chamber (Bio-Rad) and blotted in transfer buffer for $1 \mathrm{~h}$ at 100 V.

\subsection{Immunologically protein detection}

The membrane containing transferred proteins was blocked by $5 \%$ milk buffer for $1 \mathrm{~h}$ at room temperature. The primary antibodies were added to the membrane and incubated in $5 \%$ milk buffer over night at $4{ }^{\circ} \mathrm{C}$. On next day the membrane was washed with TBST 3 times for $10 \mathrm{~min}$ each at room temperature. Incubation of membrane with horseradish peroxidase conjugated secondary antibodies occurred for $1 \mathrm{~h}$ at room temperature. After 3 times TBST washing ECL solutions (Pierce) were added to the membrane according to instruction manual. Chemoluminescence was detected by Amersham Hyperfilm ${ }^{\mathrm{TM}}$ ECL (GE Healthcare).

\subsection{Co-IP (Co-immunoprecipitation)}

$5 \times 10^{6}$ untransfected cells or $24 \mathrm{~h}$ post-transfection cells were harvested by trypsination and resuspended in $1 \mathrm{ml}$ lysis buffer by vortexing and passing 10 times through a 21 gauge needle. The cell lysate was then incubated on ice for $20 \mathrm{~min}$ followed by sonication 2 times for $45 \mathrm{sec}$ each. After centrifugation at 15,000 x g for 15 min, the supernatant was recovered and splitted into two equal parts. The pellet (described as p) as well as $50 \mu 1$ of supernatant (described as s) was stored for future Western blot analyses. Furthermore, the supernatant was subjected to immunoprecipitation. One part was incubated with the fishing antibody for $1 \mathrm{~h}$ at 
room temperature whereas the second part was used as control without antibody incubation. Subsequently, pre-washed protein G agarose beads were added to both probes and mixtures rotated over night at $4{ }^{\circ} \mathrm{C}$. Beads of proteins-antibodies-mixture were collected by centrifugation, and a $50 \mu \mathrm{l}$ aliquot of the supernatant after centrifugation was taken as depletion control (described as dc). The Beads of proteins-antibodies-mixture were washed 4 times with $1 \mathrm{ml}$ of lysis buffer each. $50 \mu 1$ last wash buffer was collected (described as lw). Both aliquots were added with $50 \mu 1$ $2 \mathrm{x}$ denaturation buffer, respectively. After washing beads bound proteins were added with $50 \mu 11 \mathrm{x}$ denaturation buffer (described as $\mathrm{b}$ ). The beads of control probe were collected by centrifugation and added with $50 \mu 11 \mathrm{x}$ denaturation buffer (described as bc). The fractions $\mathrm{p}$ and $\mathrm{s}$ were added with $50 \mu 11 \mathrm{x}$ and $2 \mathrm{x}$ denaturation buffer, respectively. All fractions added with denaturation buffer were boiled for $10 \mathrm{~min}$ at 95 ${ }^{\circ} \mathrm{C}$ and ready for Western blot analyses.

\subsubsection{Cell biological methods}

\subsection{Propagation of eukaryotic cells and subculture}

All applied eukaryotic cells were cultured in suitable medium (see 2.1.7) at $37{ }^{\circ} \mathrm{C}$ and $5 \% \mathrm{CO}_{2}$.

The old medium was aspirated and the cells were washed with PBS. $0.25 \%$ trypsin was added to the cells and incubated for $3 \mathrm{~min}$ at $37^{\circ} \mathrm{C}$. After incubation trypsin was inactivated by adding fresh complete medium containing $10 \%$ FBS. Cells were resuspended with fresh medium and an aliquot of the suspension was transferred into a new flask. Then $10 \mathrm{ml}$ fresh medium was added to the transferred cells in the new flask. 


\subsection{Defrosting and freezing of eukaryotic cells}

For defrosting the cells were removed from liquid nitrogen and quickly put into the pre-warmed water bath for $3 \mathrm{~min}$ at $37{ }^{\circ} \mathrm{C}$. Then the $1.5 \mathrm{ml}$ cell-suspension was diluted with fresh medium and centrifuged for $3 \mathrm{~min}$ at $800 \mathrm{xg}$. The supernatant was discarded and the cell pellet resuspended with fresh medium. The suspension was resuspended and centrifuged for $3 \mathrm{~min}$ at $800 \mathrm{x}$ g again. $10 \mathrm{ml}$ medium was added to the cell pellet and resuspended, and removed into a new flask.

For freezing the cells were trypsinized and centrifuged for $3 \mathrm{~min}$ at $800 \mathrm{x} \mathrm{g}$. Then cells were washed with PBS and resuspended with $3 \mathrm{ml}$ freezing medium. The suspension was splitted into two equal parts and stored in labeled freezing tubes. Cells were firstly incubated for $2 \mathrm{~h}$ at $-20{ }^{\circ} \mathrm{C}$ and subsequently over night at $-80{ }^{\circ} \mathrm{C}$. Finally the cells were stored in liquid nitrogen.

\subsection{Assembly of transient transfection of cells}

Plasmid DNA was transfected into NIH3T3, MCF7 and MDA-MB-231 with Transfectin and into HEK293 with XtremeGeneHP as indicated in the provided instruction manuals. The cells were incubated for 24 to $48 \mathrm{~h}$ at $37{ }^{\circ} \mathrm{C}$ and $5 \% \mathrm{CO}_{2}$. After incubation the exogenous proteins were over-expressed and ready for future investigation.

\subsection{Assembly of stably transfected cells}

Each cell line of NIH3T3, HEK293, MCF7 and MDA-MB-231 was transfected with $O d f 2(13.8 N C)-G F P$ and empty vector $p E G F P-N 1$, respectively. $48 \mathrm{~h}$ post-transfection cells were selected under G418 control. After 8 weeks the stably transfected cells were assembled. 


\begin{tabular}{|c|c|}
\hline Cell line & G418 concentration in medium \\
\hline NIH3T3 & $400 \mu \mathrm{g} / \mathrm{ml}$ \\
\hline HEK293 & $400 \mu \mathrm{g} / \mathrm{ml}$ \\
\hline MCF7 & $500 \mu \mathrm{g} / \mathrm{ml}$ \\
\hline MDA-MB-231 & $600 \mu \mathrm{g} / \mathrm{ml}$ \\
\hline
\end{tabular}

Tab. 2.11: G418 concentrations of different cell culture media.

\subsection{Immunocytology}

Untransfected or $24 \mathrm{~h}$ post-transfection cells cultured on coverslips were washed 3 times with PBS for 5 min each and fixed in $3.7 \%$ PFA in PBS for 20 min at $4{ }^{\circ} \mathrm{C}$. Then the cells were permeabilized for 10 min with $0.3 \%$ Triton X-100 in PBS at room temperature and blocked for $1 \mathrm{~h}$ with PBT. Primary antibodies were added to cells and incubated for $1 \mathrm{~h}$ at $37{ }^{\circ} \mathrm{C}$. Cells were washed with PBS for 3 times $5 \mathrm{~min}$ each. Then the secondary antibodies were added to cells and incubated for $45 \mathrm{~min}$ at $37{ }^{\circ} \mathrm{C}$. The DNA was counterstained with DAPI. After 3 times PBS washing DAKO mounting medium (DAKO) was added to cells and cells were sealed between coverslip and object slide by nail polish. Images were taken by confocal microscopy (LSM 510, Zeiss) and processed using Adobe Photoshop 7.0.

\subsubsection{Specific methods of Odf1-deficient mice investigation}

\subsubsection{Generation of targeting vector}

All mouse experiments were reviewed and approved by the local ethics commission. Licensing for animal experiments was obtained by the Institute of Human Genetics. A genomic Odf1 cosmid clone (121 J 1787Q3; 129/Ola) was obtained from the Resource Centre of the German Human Genome Project at the Max-Planck-Institute for Molecular Genetics (Berlin, Germany) and mapped for restriction enzyme recognition sites. To assemble the targeting vector, an XbaI fragment containing $\sim 4$ $\mathrm{kb}$ of the $5^{`}$ region, including the promoter region, as well as exon 1, was subcloned 
into pGEMT, and exoIII deletions, starting from the 3' region, were performed. A fragment of $\sim 3 \mathrm{~kb}$ consisting of the upstream genomic region of the Odf1 gene was first subcloned via XbaI / EcoRI into pBluescript, followed by NotI / XhoI cloning into pPNT-M1. The 3.8 -kb PstI intron fragment was first subcloned into pBluescript, followed by XbaI / EcoRI directional cloning into pPNT-M1. The resulting targeting vector (Fig. 3.1A) was sequenced to verify the correct orientations of both fragments. The targeting construct was linearized at the unique NotI site before electroporation.

\subsubsection{Generation of the 3`external probe and Southern blot hybridization}

The 3`external hybridization probe was generated by PCR amplification using the primer pair Odf1SPst and Odf1SHindIII out of a genomic Odfl clone and cloned into pGEMT.

For Southern blot hybridization, genomic DNA was extracted from ES cells, digested with HindIII, electrophoretically separated on agarose gels, and transferred onto Hybond N membranes (Amersham, Freiburg, Germany) by capillary blotting. The 3' external probe was labeled with $\left[{ }^{32} \mathrm{P}\right] \mathrm{dATP}$ by the random hexanucleotide primer method (AP Feinberg and B. Vogelstein, 1983), and hybridized over night in 5x SSPE, 5x Denhardt's solution, $0.1 \%$ SDS, and $100 \mu \mathrm{g}$ of denatured salmon sperm DNA / ml (E. Southern, 1975) at $65^{\circ} \mathrm{C}$. Filters were washed 2 times at room temperature in $2 \mathrm{x}$ SSC, then in $1 \mathrm{x}$ SSC containing $0.1 \%$ SDS, and finally in $0.1 \mathrm{x}$ SSC containing $0.1 \%$ SDS at hybridization temperature.

\subsubsection{ES cell culture and generation}

ES cell line R1 (provided by A. Nagy, Toronto, Ontario, Canada) was cultured as described by A. Joyner (A. Joyner, 1993). Trypsinized and resuspended ES cells were mixed with $50 \mathrm{mg}$ of linearized target vector and electroporated at $250 \mathrm{~V}$ and $500 \mu \mathrm{F}$ using a Bio-Rad gene pulser apparatus (Bio-Rad). Cells were plated in nonselective 
medium containing leukemia inhibitory factor in the presence of G418-resistant embryonic mouse fibroblasts. Selection for homologous recombined ES cells was carried out with medium containing $350 \mu \mathrm{g} / \mathrm{ml} \mathrm{G} 418$ and $2 \mu \mathrm{mol} / \mathrm{l}$ ganciclovir. After 10 days of selection, individual drug-resistant clones were picked into 24-well trays. Three days later, individual recombinant ES clones were replicated into 24-well trays for freezing and for isolation of genomic DNA. A total of 10 to 15 compacted recombinant ES cells were microinjected into 3.5-day-old embryos of the C57BL/6 mouse strain (A. Joyner, 1993). The chimeric male mice generated were mated with C57BL/6 females to produce offspring.

Heterozygous $O d f 1$-deficient mice generated by mating of chimeric male mice with C57BL/6 females were backcrossed with mice from inbred strain $129 \mathrm{~Sv}$ to reduce C57BL/6 background genes. Heterozygous offspring were backcrossed with 129Sv mice up to generation N7 that is then supposed to be isogenic.

\subsubsection{Genotyping}

Genomic DNA from tail tip biopsy was extracted with Viagen DirectPCR-Tail and proteinase $\mathrm{K}$ digestion as indicated in the provided instruction manual. Genotyping was performed with the following primer pairs: Odf1-I and Neomy for detection of the neomycin cassette in the recombined allele, Odf1-5 ko and Odf1-I for differentiation between the wild-type allele of $\sim 1.5 \mathrm{~kb}$ and the recombined allele of $\sim 2.4 \mathrm{~kb}$, and Odf1-I and Odf1-N for detection of the wild-type allele. A first PCR was performed with the primer pair Odf1-I / Neomy for detection of the recombined allele and a second PCR with the primer pair Odf1-I / Odf1-N for detection of the wild-type allele and to differentiate between heterozygous and homozygous mice. Both PCR amplifications were performed with an initial denaturation step at $95^{\circ} \mathrm{C}$ for $3 \mathrm{~min}$, followed by 35 cycles at $95^{\circ} \mathrm{C}$ for $30 \mathrm{sec}$, annealing at $49^{\circ} \mathrm{c}$ for $30 \mathrm{sec}$, and elongation at $72^{\circ} \mathrm{C}$ for $1 \mathrm{~min}$, and a final extension step at $72^{\circ} \mathrm{C}$ for $8 \mathrm{~min}$. Mango Taq DNA polymerase was used for these two PCRs. 


\subsubsection{Fertility test}

The fertility of the Odf1-deficient males on a mixed background (C57BL/6 x 129/Sv) was investigated by mating with wildtype females. Mating tests were performed with 11 homozygous male mice $\left(O d f 1^{--}\right)$for at least 3 months. As a control group, six of their male littermates with a heterozygous genotype $\left(O d f 1^{+-}\right)$were mated with wild-type females. Females were checked for the presence of vaginal plugs and/or pregnancy. Pregnant females were removed to holding cages to give birth. The numbers and size of litters sired by each group of males were determined.

The insemination capacity of the heterozygous Odfl-deficient males on isogenic background (129/Sv; generation N7) was also investigated by mating with wild-type females. Females were checked for the presence of a vaginal plug. Liquid was isolated from uteri and inspected for the presence of sperm.

\subsubsection{Reverse transcriptase PCR on genes of Odf1-deficient mice}

Total RNA was extracted from testes of wild-type, heterozygous, and homozygous Odf1 knock out mice, respectively, using peqGOLD RNAPure and digested with RQ1 DNase. RNA was reverse transcribed using RevertAid H Minus First Strand cDNA synthesis kit and oligo $(\mathrm{dT})_{18}$ primer, followed by PCR. The corresponding PCR parameters of each primer pair (annealing temperature, elongation time, and size of amplified fragments) are shown in table 2.12. PCR amplification was performed with an initial denaturation step at $95^{\circ} \mathrm{C}$ for $3 \mathrm{~min}$, followed by 36 cycles at $95^{\circ} \mathrm{C}$ for 30 sec, annealing for $30 \mathrm{sec}$, and elongation at $72^{\circ} \mathrm{C}$, and a final extension step at $72^{\circ} \mathrm{C}$ for 8 min. PCR amplification of $O d f 1$ was applied with Mango Taq DNA polymerase and all the rest PCRs were performed with Taq polymerase. 


\begin{tabular}{|c|c|c|c|c|}
\hline Target gene & Primer & $\begin{array}{c}\text { Annealing } \\
\text { Temprature } \\
\left({ }^{\circ} \mathrm{C}\right)\end{array}$ & Elongation time & $\begin{array}{l}\text { Product size } \\
\text { (bp) }\end{array}$ \\
\hline \multirow[t]{2}{*}{$S Y C P 3$} & MSYCP35 Kpn1 & \multirow[t]{2}{*}{52} & \multirow[t]{2}{*}{$1 \mathrm{~min}$} & \multirow[t]{2}{*}{900} \\
\hline & MSYCP3r & & & \\
\hline \multirow[t]{2}{*}{ Stra8 } & Stra8 for & \multirow[t]{2}{*}{56} & \multirow[t]{2}{*}{$30 \mathrm{~s}$} & \multirow[t]{2}{*}{444} \\
\hline & Stra8 rev & & & \\
\hline \multirow[t]{2}{*}{ HANP1/H1T2 } & Hanp1-H1T2-for & \multirow[t]{2}{*}{50} & \multirow[t]{2}{*}{$1 \mathrm{~min}$} & \multirow[t]{2}{*}{800} \\
\hline & Hanp1-H1T2-rev & & & \\
\hline \multirow[t]{2}{*}{$M I S$} & MMISf & \multirow[t]{2}{*}{56} & \multirow[t]{2}{*}{$30 \mathrm{~s}$} & \multirow[t]{2}{*}{315} \\
\hline & MMISr & & & \\
\hline \multirow[t]{2}{*}{ Ins 13} & mIns13f & \multirow[t]{2}{*}{56} & \multirow[t]{2}{*}{$30 \mathrm{~s}$} & \multirow[t]{2}{*}{543} \\
\hline & mIns13r & & & \\
\hline \multirow[t]{2}{*}{ Odf2 } & 138NCEcoRI & \multirow[t]{2}{*}{59} & \multirow[t]{2}{*}{$1 \mathrm{~min}$} & \multirow[t]{2}{*}{$822 / 677$} \\
\hline & DH1rev & & & \\
\hline \multirow[t]{2}{*}{ Odf1 } & Odf1-N & \multirow[t]{2}{*}{52} & \multirow[t]{2}{*}{$1 \min$} & \multirow[t]{2}{*}{800} \\
\hline & Odf1-C & & & \\
\hline
\end{tabular}

Tab. 2.12: Primers application of RT-PCR in Odf1 knock out mice.

\subsubsection{Histology and immunocytology of mouse tissues}

Testes were alternatively fixed in 4\% PFA in PBS or in Bouin's solution and embedded in paraffin. Then, 4- $\mu \mathrm{m}$ sections were cut and placed onto Superfrost ${ }^{\circledR}$ slides (Thermo Scientific). Drying of probes was performed by incubation for over night at $55{ }^{\circ} \mathrm{C}$. The probes were deparaffinized with xylol two times for 3 min each and last time for $5 \mathrm{~min}$ and then rehydrated in ethanol with decreasing concentration from $100 \%, 90 \%, 80 \%$, and down to $70 \%$ for $2 \mathrm{~min}$, each. After washing with milliq $\mathrm{H}_{2} \mathrm{O}$, the probes were stained with hematoxylin for 1-2 min and then immediately put into milliq $\mathrm{H}_{2} \mathrm{O}$ for short washing, and afterward washed with flowing tap water for 5-15 min. Furthermore, the probes were quickly put into acid-ethanol and removed from the solution, this step was repeated for 3-4 times. Subsequently, the probes were quickly put into ammoniac water and removed from the solution, this step was also repeated for 3-4 times. Then the probes were shortly washed with tap water. Then they were stained with eosin for 1 min. After washing with water the probes were dehydrated in ethanol with increasing concentration from $70 \%, 80 \%, 90 \%$ and up to 
$100 \%$ for $1 \mathrm{~min}$, each. The probes were put into xylol for $10 \mathrm{~min}$. After all, the sections were embedded with Eukitt quick-hardening mounting medium (FLUKA). For immunocytology, epididymides were minced in PBS, and the cell suspension was transferred onto Superfrost plus slides by centrifugation at 1,000x $g$ for $5 \mathrm{~min}$. The cells were fixed in 3.7\% PFA in PBS, blocked in PBT for 30 min, and incubated with anti- $\alpha$-tubulin antibody for $1 \mathrm{~h}$. The primary antibody was detected by Cy3-labeled anti-mouse IgG, the DNA was counterstained with DAPI, and the acrosome was decorated with PL-FITC. Images were taken by confocal microscopy (LSM 510, Zeiss) and processed using Adobe Photoshop 7.0.

\subsubsection{Electron microscopy}

Testes and epididymides were fixed in $1 \%$ glutaraldehyde in $0.2 \mathrm{M}$ phosphate buffer, postfixed with $2 \%$ osmium tetroxide, and embedded in epoxy (Epon) resin. Selected areas were sectioned and examined by transmission electron microscopy.

\subsubsection{Sperm analysis}

Heterozygous and homozygous Odfl-deficient male mice of the mixed background, wild-type males of C57BL/6 strain, as well as heterozygous Odfl-deficient male mice of the isogenic background (generation N7), and wildtype males of 129/Sv strain, were used for sperm analyses. Epididymides were collected and dissected in Tyrode's medium. Sperm number in corpus and cauda epididymis was determined using the Neubauer cell chamber.

For acrosome reaction, spermatozoa were capacitated for $1.5 \mathrm{~h}$ in Tyrode's medium and then incubated for $5 \mathrm{~min}$ at $37^{\circ} \mathrm{C}$ and $5 \% \mathrm{CO}_{2}$ with Tyrode's medium plus $20 \mu \mathrm{M}$ calcium ionophore. For the determination of the percentage of sperm that had undergone an acrosome reaction, sperm were fixed and stained with Coomassie brilliant blue as described by C. Thaler (C. Thaler, 1995). At least 200 spermatozoa 
from each male were examined for the presence or absence of the characteristic dark blue crescent.

\subsubsection{In vitro fertilization (IVF) with heterozygous Odf1-deficient males on isogenic background (129/Sv; generation N7)}

Adult female mice were superovulated by intraperitoneal injections of 5U PMSG. 48 $\mathrm{h}$ later 5U HCG was injected intraperitoneally. Oocytes from superovulated females were isolated 15-17 $\mathrm{h}$ after HCG injection. Isolated oocytes were freed from cumulus cells and zona pellucida by incubation in hyaluronidase $(300 \mu \mathrm{g} / \mu \mathrm{l})$ for $10 \mathrm{~min}$ at 37 ${ }^{\circ} \mathrm{C}$ followed by incubation in Tyrode's solution ( $\mathrm{pH}$ 2.7) for $10-20 \mathrm{sec}$. Oocytes were then transferred into IVF Cook medium previously equilibrated over night at $37{ }^{\circ} \mathrm{C}$ and $5 \% \mathrm{CO}_{2}$. In vitro fertilization took place in equilibrated Cook medium containing isolated epididymal spermatozoa for $6 \mathrm{~h}$ at $37{ }^{\circ} \mathrm{C}$ and $5 \% \mathrm{CO}_{2}$. Oocytes were then washed in pre-equilibrated Cook medium and incubated over night at $37{ }^{\circ} \mathrm{C}$ and $5 \%$ $\mathrm{CO}_{2}$. Degenerated oocytes and embryos were counted $24 \mathrm{~h}$ after IVF.

\subsubsection{Sperm motility analysis}

Epididymides of three wild-type, heterozygous, and homozygous Odfl-deficient males, respectively, were dissected in IVF Cook medium. Spermatozoa were allowed to swim out of the epididymides and incubated for $1.5 \mathrm{~h}$ at $37^{\circ} \mathrm{C}$. A total of $13 \mu \mathrm{l}$ of the sperm suspension was transferred to the incubation chamber, which was set at a temperature of $37{ }^{\circ} \mathrm{C}$. Sperm movement was quantified using the CEROS computer-assisted semen analysis system (version 10; Hamilton Thorne Research, Beverly, MA). Then, 1,000 to 3,000 spermatozoa from each individual were analyzed using the following parameters: negative phase-contrast optics; recording, 60 frames/s; minimum contrast, 60; minimum cell size, 6 pixels; straightness (STR) threshold, $\geq 50 \%$; cutoff of the average path velocity (VAP) and straight line velocity (VSL), 25 
and $30 \mu \mathrm{m} / \mathrm{s}$, respectively; minimum progressive average path velocity (VAP), 75 $\mu \mathrm{m} / \mathrm{s}$; slow cells motile, no (this limit avoids counting sperm moved by others or Brownian motion and low-velocity non-progressive cells); and minimum static contrast, 15 pixels.

For statistical analysis, the frequencies of the six sperm motility parameters VAP, VSL, VCL, ALH, BCF, and STR were examined. Because all analyzed parameters were significantly different from normal distribution (Shapiro-Wilk's W test) and could not be normalized by any transformation, the nonparametric alternative for $t$ test, the Mann-Whitney $U$ test, was used. For statistical testing, sperm motility measurements of each parameter were pooled for mouse type. Statistical analyses were performed by Statistica 9 (StatSoft, Inc., Tulsa, USA).

\subsubsection{Specific methods of SPAG4 protein investigation}

\subsubsection{Subcloning of Spag4 cDNA}

The 3' region of Spag4 was amplified out of mouse testis cDNA using primer pair SPAG4 C KpnI and Spag4 650 for. The PCR product of $\sim 650$ bp was isolated and mixed with Spag4 cDNA clone IRAMp995J1116Q (imaGenes, Germany) which encompasses $\sim 650$ bp of the 5 region of full length Spag4. A PCR reaction was performed using primer pair SPAG4 C KpnI and SPAG4 N XbaI. The PCR product of $\sim 1.2 \mathrm{~kb}$ was eventually subcloned into modified pcDNA3.1/myc-His(-) C digested with KpnI / XbaI. The final clone yielded a protein that was tagged with HA at its $\mathrm{N}$-terminal end and with Myc at its C-terminal end. N-terminal and C-terminal truncated Spag4 constructs were generated by PCR amplification and finally by subcloning into modified pcDNA3.1HA/myc-His(-) C. N-terminally truncated Spag4-C was amplified with primer pair SPAG4N2XbaI and SPAG4 C KpnI, and C-terminally truncated Spag4- $N$ was generated using primer pair SPAG4 N XbaI and SPAG4C2KpnI. Both constructs were tagged with $H A$ at their N-terminal ends. EYFP-Spag4 was generated by amplification of EYFP using primer pair EYFP-C 
$\mathrm{XbaI}$ and EYFP-N SpeI and ligation to the N-terminal end of Spag4. Odf1 was fused to $E C F P$ in $p E C F P-N 1$. Sequencing always revealed correct reading frames.

\subsubsection{Reverse transcriptase PCR on Spag4}

Total RNA was prepared from mouse tissues as well as from different cell lines (ES, SSC, and F9) using peqGOLD TriFast reagent. RNA was first digested with RQ1 DNase. Then cDNA was generated using RevertAid H Minus First Strand cDNA synthesis kit and oligo(dT) ${ }_{18}$ primer. SPAG4 C KpnI and Spag4 650 for primers were used for RT-PCR reaction to amplify SPAG4. As control, GAPDH (glyceraldehyde-3-phosphate dehydrogenase) was amplified using primers GAPDHfor and GAPDHrev.

\subsubsection{In situ proteinase $K$ digestion}

NIH3T3 cells were transfected with $H A-S p a g 4-M y c$ tagged at the N-terminus with $H A$ and at the C-terminus with Myc. $24 \mathrm{~h}$ post-transfection cells were subjected to in situ proteinase $\mathrm{K}$ digestion. In brief, cells were washed two times with ice-cold PBS, scraped in PBS and transferred into plastic tubes. Cells were then either incubated in KHM buffer containing $4 \mu \mathrm{g} / \mathrm{ml}$ proteinase $\mathrm{K}$, or in KHM buffer containing $0.5 \%$ Triton $\mathrm{X}-100$ and $4 \mu \mathrm{g} / \mathrm{ml}$ proteinase $\mathrm{K}$ for $45 \mathrm{~min}$ at room temperature. Thereafter, cells were sedimented by centrifugation and lysed in lysis buffer by passing through a 20 -gauge needle. Finally the cells were centrifuged and supernatant as well as pellet was added with denaturation buffer, boiled for $10 \mathrm{~min}$ at $95^{\circ} \mathrm{C}$. The denatured probes were ready for future Western blot analyses.

\subsubsection{Immunocytology on testicular suspensions}

Cell suspensions from testes of wild-type or $O d f 1^{-/-}$mice were prepared in PBS and 
transferred onto superfrost slides. Specimens were fixed in $3.7 \%$ PFA in PBS, permeabilized in $0.3 \%$ Triton X-100 in PBS for $10 \mathrm{~min}$, and blocked for $1 \mathrm{~h}$ in PBS containing $1 \% \mathrm{BSA}$ and $0.3 \%$ Triton X-100. Immunocytology was performed with anti-SPAG4 antibody and anti- $\alpha$-tubulin antibody over night at $4{ }^{\circ} \mathrm{C}$.

\subsubsection{Co-IP with transfected cells and testicular tissue}

NIH3T3 or HEK293 cells were either transfected with expression plasmids encoding different regions of Spag4 or Odf1, or with two different full lengths Spag4 constructs. Coding sequences were fused to diverse tags: HA-Spag4-C encoding the C-terminal end of Spag4 fused to HA, HA-Spag4-N encoding the N-terminal end of Spag4 fused to $H A$, full length Spag4 either fused to $H A$ / Myc (HA-Spag4-Myc) or to EYFP at its $\mathrm{N}$-terminal end (EYFP-Spag4-Myc). Odf1 was fused to ECFP. 24 h post-transfection cells were subjected for Co-IP.

Co-IP with testis tissue was performed essentially as described in 2.2.1.3.5 with the following modifications. Testis was frozen in liquid nitrogen and ground in a mortar. The cell lysate was prepared by resuspension in PBS containing 1\% Nonidet P40, 100 $\mu \mathrm{g} / \mathrm{ml} \mathrm{PMSF}$, and $1 \mu \mathrm{g} / \mathrm{ml}$ protease inhibitors and then sonicated 3 times for $45 \mathrm{sec}$ each. The supernatant was obtained by centrifugation for $15 \mathrm{~min}$ at 1,000x $\mathrm{g}$.

\subsubsection{Specific methods of ODF2 protein investigation}

\subsubsection{Reporter gene assay}

Reporter gene OT contains firefly-luciferase under control of promoter with Tcf4 binding site. Once the complex containing $\beta$-catenin and its co-factor Tcf4 binds to the Tcf4 binding site, the reporter gene is immediately activated. Subsequently the luciferase activity is measured by luminometer TriStar LB 941 (Berthold Technologies, Germany). In contrast to $O T$, as negative control $O F$ vector has a mutated Tcf4 binding site. As internal control renilla-luciferase gene ( $p h R L)$ was 
considered to control transfection efficiency. The influence of ODF2 on canonical Wnt signaling pathway or rather on $\beta$-catenin was investigated by co-transfection of Odf2 (13.8NC)-GFP and $\beta$-catenin. $24 \mathrm{~h}$ post-transfection cells were scratched from the bottom of the wells of 12 -well plate and centrifuged for $10 \mathrm{~min}$ at $800 \mathrm{x} \mathrm{g}$. The pellet was resuspended and lysed in $30 \mu \mathrm{l}$ lysis buffer for $30 \mathrm{~min}$ at room temperature. Then the suspension was centrifuged at 13,000x g for $1 \mathrm{~min}$ at room temperature. The supernatant was transferred into well of 96-well plate and subsequently added with 50 $\mu 1$ of firefly-luciferase substrate (buffer A). After firefly-luminescence measurement the reaction was prevented by adding $50 \mu$ renilla-luciferase substrate (buffer B). Renilla-luminescence was also measured by luminometer.

All experiments were performed in triplicate. The value of firefly-luminescence was divided by renilla-luminescence and then multiplied by 1000 to obtain relative firefly-luciferase activity. The values measured by luminometer are demonstrated in the supplement.

\subsubsection{Micro injection into embryos of Xenopus laevis}

The eggs of Xenopus laevis were collected and mixed with the testis mixture. After the in vitro fertilization the eggs were dejellied. After dejellying, the fertilized eggs were transferred to $0.1 \mathrm{x}$ MBS containing $1 \%$ Ficoll prior to injection. At the 3.stage (4-cell) the embryos were injected in its marginal zone of ventral area where the nature $X w n t-8$ is enriched. The injection was performed for every time with three groups: only Xwnt- $8, X w n t-8$ and $O d f 2$, and only $O d f 2$. For co-injection of Xwnt- 8 and Odf2 different RNA amount combinations of both genes were carried out. 100 to 200 embryos were injected for each combination of co-injection or single injection to implement statistic analysis. After injection, the embryos were incubated at $14^{\circ} \mathrm{C}$ and had to be controlled twice a day by microscopy. Only living embryos were allowed to stay further and transferred into fresh $0.1 x$ MBS buffer. In about 4 days the embryos entered into tadpole stages, and the tadpoles with secondary dorsal axis were counted. 


\subsubsection{Quantitative real time PCR}

HEK293 cells were transiently transfected with following constructs: $13.8 N C-G F P$, 13.8NCAGFP, $p E G F P-1, p E G F P-N 1, s h O D F 2$, respectively. Total RNA was extracted from these transiently transfected cells, untransfected cells and cells stably transfected with $13.8 N C$-GFP using peqGOLD RNAPure and digested with RQ1 DNase. RNA was reverse transcribed using RevertAid H Minus First Strand cDNA synthesis kit and oligo $(\mathrm{dT})_{18}$ primer. PCR was performed with primers hHPRT1_2f_390/hHPRT1_2r_631 and hCmyc_2f_344 / hCmyc_2r_615. $10 \mu$ final reaction volume contains $0.3 \mu \mathrm{l}$ forward primer $(10 \mathrm{pmol} / \mu \mathrm{l}), 0.3 \mu \mathrm{l}$ reverse primer (10 pmol/ $\mu \mathrm{l}$ ), $1 \mu \mathrm{l}$ cDNA (equivalent of $20 \mathrm{ng} \mathrm{RNA}$ ), $5 \mu \mathrm{liQ}{ }^{\mathrm{TM}} \mathrm{SYBR}^{\circledR}$ Green Supermix and $3.4 \mu \mathrm{l}$ nuclease-free $\mathrm{H}_{2} \mathrm{O}$. The PCR was performed using the standard reaction program of $\mathrm{CFX} 96^{\mathrm{TM}}$ Real-Time System (Bio-Rad) with annealing temperature at $58{ }^{\circ} \mathrm{C}$ and elongation for $30 \mathrm{sec}$.

\subsubsection{Wound healing assay}

NIH3T3, MCF7 and MDA-MB-231 cell lines were examined using wound healing assay. Untransfected cells, cells stable transfected with empty vector $p E G F P-N 1$ and cells stably transfected with $13.8 N C$-GFP were applied for each cell line. $100 \%$ confluent cells were scratched and cultured in serum starvation medium. The width of wound was measured after certain hours and analysed with ImageJ software. 


\section{Results}

\subsection{ODF1 protein}

\subsubsection{ODF1 protein is essential for tight linkage of sperm head to tail and male fertility in mice}

\subsubsection{The generation of mice with disruption of the Odf1 gene}

The Odfl gene consists of two exons separated by one large intron. Transcription and translation start sites are both in exon 1 (Burfeind and Hoyer-Fender, 1991; Burfeind et al., 1993; Gastmann et al., 1993). The targeting vector used to disrupt Odf1 was designed with a neomycin cassette to replace exon 1 and the promoter region (Fig. 3.1 A). After electroporation of the linearized targeting vector into mouse ES cells recombinant colonies were established by selection with G418 and ganciclovir. Next, Southern blot hybridization with HindIII digested genomic DNA of individual ES cell clones and $3^{`}$ external probe was performed. Out of 96 ES cell clones tested, 20 clones showed the expected hybridization pattern of a band of $\sim 5.4 \mathrm{~kb}$ that refers to the wild-type allele and a band of $\sim 7.2 \mathrm{~kb}$ that refers to the homologous recombined allele (Fig. 3.1 B). One ES cell clone that showed the expected hybridization pattern after repeated Southern blot hybridization with the external 3 ' probe was chosen for microinjection into blastomeres of 3.5-day-old embryos of C57BL/6 mice. After reimplantation of embryos, several chimeras were born, most of them with a high chimerism (>90\%). Male chimeras mated with C57BL/6 females transmitted the targeted allele to their offspring. Offspring were genotyped by PCR on genomic DNA isolated from tail tip biopsy specimens using primer pair Neomy/Odf1-I (Fig. 3.1 C). Heterozygous mice were crossed with $\mathrm{C} 57 \mathrm{BL} / 6$ mice to establish the heterozygous $O d f 1^{+/-}$line. Eventually, heterozygous male and female mice were crossed to generate Odf1-deficient mice. Genotyping was generally performed on tail tip biopsy specimens using the primer pair Neomy/Odf1-I for detection of the recombined allele and with primer pair Odf1-N/Odf1-I for detection of the wild-type allele (Fig. 3.1 C). 


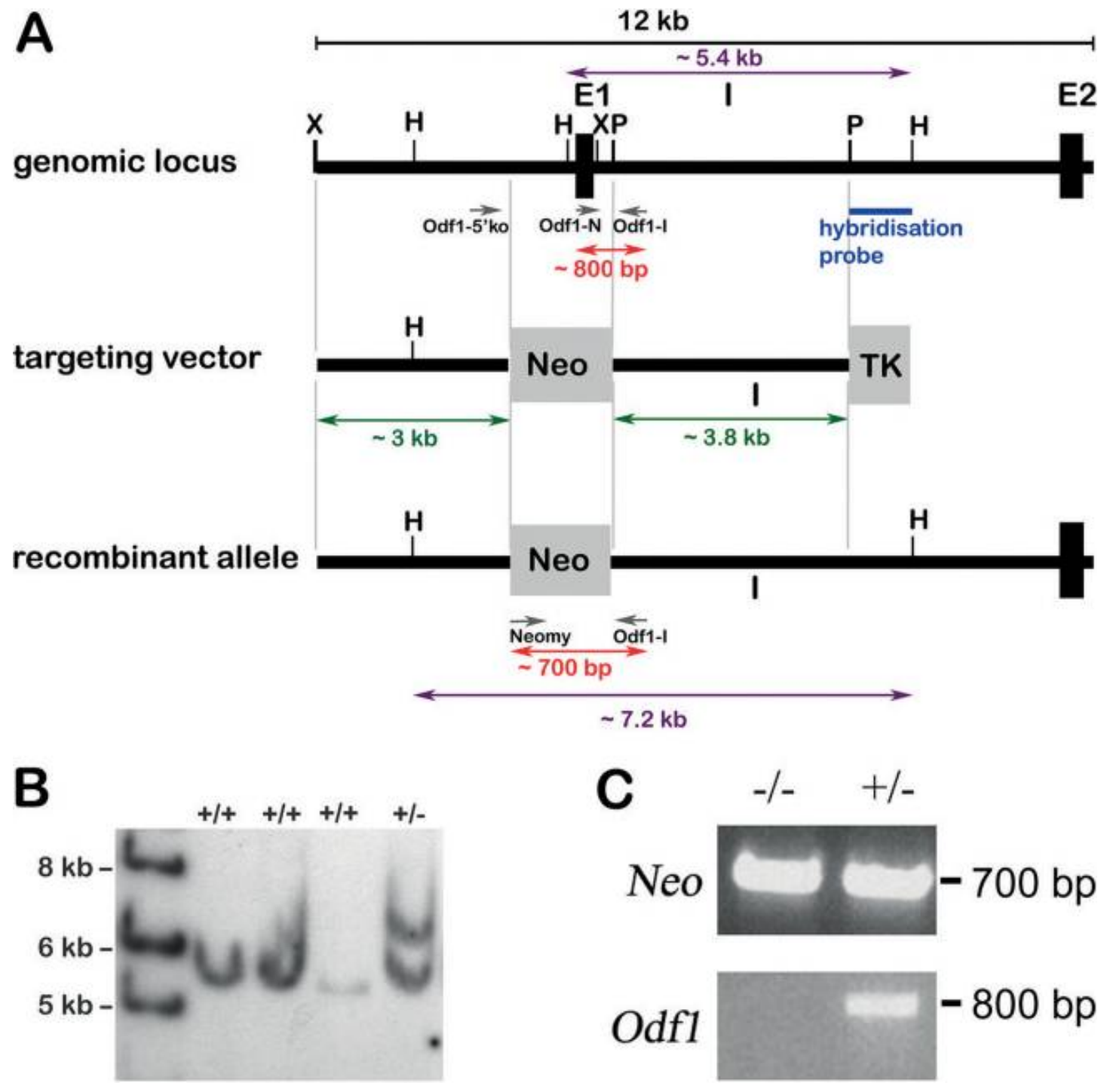

Fig 3.1: Targeted disruption of the promoter region and of exon 1 of the Odf1 gene. (A), structure of the wild-type allele, targeting vector, and recombinant allele are shown, together with relevant restriction sites, primer positions, and external hybridization probe position. The targeting vector consists of $5^{\wedge}$ upstream region $(\sim 3 \mathrm{~kb})$ and intronic sequences $(\sim 3.8 \mathrm{~kb})$ flanking the pgk-neomycin selection cassette (Neo). TK, thymidine kinase cassette; X, XbaI; H, HindIII; P, PstI; E1, exon 1; E2, exon 2; I, intron; Odf1-5 ko, Odf1-I, Odf1-N, and Neomy, primers used for genotyping. Southern blot hybridization of HindIII-digested genomic DNA with the 3' external probe resulted in an $\sim 5.4-\mathrm{kb}$ fragment of the wild-type allele and an $\sim 7.2-\mathrm{kb}$ fragment of the recombined allele. Genotyping with primer pair Odf1-N/Odf1-I resulted in an 800-bp fragment of the wild-type allele, and genotyping with the primer pair Neomy/Odf1-I resulted in an $~ 700$-bp 
fragment of the recombined allele. (B), Southern blot hybridization of HindIII-digested genomic DNA isolated from individual ES cell colonies generated by electroporation with the targeting construct, followed by selection with G418 and ganciclovir. Hybridization with the 3' external probe to a fragment of $\sim 7.2 \mathrm{~kb}$ revealed homologous recombination. The fragment of the wild-type allele is shown at $\sim 5.4 \mathrm{~kb} .+/+$, no homologous recombination; +/-, homologous recombination. (C), genotyping using the primer pair Neomy/Odf1-I for detection of the recombined allele $(\mathrm{Neo})$ and the primer pair Odf1-N/Odf1-I for detection of the wild-type allele (OdfI) in homozygous (-/-) and heterozygous (+/-) mice.

\subsubsection{Odf1-deficient male mice are infertile}

Heterozygous and homozygous Odfl-deficient male mice were first inspected regarding their overall morphological features taking into consideration testes weight related to body length and weight. However, no significant difference between both groups could be found. Phenotypic differences mostly rely on individual deviations irrespective of the presence of ODF1. Testis weight especially was related to body length and weight but not to the presence or absence of ODF1. Therefore, Odf1 deficiency did not obviously influence these morphological criteria.

However, the infertility in homozygous $O d f 1$-deficient male mice has been observed, whereas fecundity was not impaired in $O d f 1$-deficient female mice or in heterozygous male mice. To further confirm the infertility of $O d f 1^{-/-}$-male mice, $11 O d f 1^{-/-}$-male mice were mated with wild-type females. Over a time period of at least 3 months, none of these pairings gave birth to offspring or even resulted in pregnancy. In contrast, their heterozygous $O d f 1^{+/}$male littermates $(n=6)$ gave rise to offspring generally after about 4 weeks of mating with wild-type females. In test matings, no sperm in the uterus of the female mouse was found when mated to Odfl-deficient male, although the vaginal plug inspection was positive. The litter size of matings of wild-type C57BL/6 females with heterozygous $O d f 1^{+-}$males $(n=29$ litters; average litter size of 6.7) was comparable to that of wild-type breeding (litter size of 6.2). 
Furthermore, offspring of heterozygous males have sex ratios of approximately Mendelian ratio (57 male and 43 female pups were born), suggesting that OdfI deficiency in the heterozygote state did not impair embryonic development. In addition, $O d f 1$-deficient female mice $\left(O d f 1^{-/}\right)$are not impaired in fertility (Tab. 3.1). Therefore the Odf1-deficient mouse line was established by mating of Odf1-deficient females $\left(O d f 1^{-/}\right)$with heterozygous males.

\begin{tabular}{|c|c|c|c|c|c|}
\hline Genotype & $\begin{array}{c}\text { Male } \\
\text { fertility }\end{array}$ & $\begin{array}{c}\text { Average } \\
\text { litter size } \\
\text { (males) }\end{array}$ & $\begin{array}{c}\text { Mean testis / body weight } \\
\mathbf{( x 1 0 ^ { - 3 } ) \pm \text { SD }}\end{array}$ & $\begin{array}{c}\text { Female } \\
\text { fertility }\end{array}$ & $\begin{array}{c}\text { Average } \\
\text { litter size } \\
\text { (females) }\end{array}$ \\
\hline \hline$+/+$ & $28 / 28$ & 6.2 & Not determined & $28 / 28$ & 6.2 \\
\hline$+/-$ & $29 / 29$ & 6.7 & $3.1 \pm 0.37(\mathrm{n}=3)$ & $19 / 19$ & 5.8 \\
\hline$-/-$ & $11 / 0$ & 0 & $2.92 \pm 0.31(\mathrm{n}=6)$ & $10 / 10$ & 5.2 \\
\hline
\end{tabular}

Tab. 3.1: Fertility, fecundity, and testis weight of wild-type and Odf1 mutant mice. To test fertility, sexually mature mice were bred for at least 3 months. Litter size was recorded for each mating. Testis weight and body weight were measured from mice older than 5 months of age.

\subsubsection{Spermatogenesis in Odf1-deficient mice}

Histological analyses of testes from heterozygous and homozygous Odfl-deficient adult mice revealed that spermatogenesis proceeded normally in all heterozygous mice $(n=4)$ (Fig. 3.2 B g). However, three different phenotypes were observed in $O d f 1^{--}$mice. One of nine homozygous mice analyzed showed an obvious wild-type phenotype with elongating spermatids in the seminiferous tubules (Fig. $3.2 \mathrm{~B} \mathrm{~h}$ ). Six mice had displaced germ cells in the lumen (Fig. 3.2 B, arrow in panel i), and two mice had multinuclear cells in the lumen (Fig. 3.2 B, arrow in panel j). Mature sperm were barely found.

To investigate whether spermatogenesis is also affected, I inspected testis sections of one to three individuals each aged 10, 15, and 19 days post partum (dpp). Histological analyses of testis sections from $10 \mathrm{dpp}$ onward revealed no obvious differences 
between heterozygous and homozygous Odfl-deficient mice (Fig. 3.2 A). Spermatogenesis proceeded normally as previously described (Nebel et al., 1961). Meiosis starts around day 10, and early round spermatids are present $\sim 10$ days later. Fig. 3.2 A (b, d, and f) demonstrates that the spermatogenesis of Odf1-deficient males proceeded normally, and at 19-dpp round spermatids were observed in heterozygous as well as in homozygous mutants (arrows in Fig. 3.2 A, e and f). The early elongating spermatid stage then is reached at about day 26 and elongating spermatids were also detected in most of the Odfl-deficient testes (Fig. 3.2 B, h and i).

Furthermore, I investigated testis composition and spermatogenic progression by reverse transcription-PCR (RT-PCR) of marker genes. Insl3 was chosen as a marker gene for Leydig cells, Mis (Müllerian inhibiting substance) as a marker gene for Sertoli cells, Stra8 as a marker gene for spermatogonia, Sycp3 (Scp3, synaptonemal complex protein 3) as a marker gene for meiosis, Hanp1/ H1T2 as a marker gene to monitor spermiogenic progression, and $O d f 2$ as a ubiquitously expressed gene that is upregulated during spermiogenesis (Adham et al., 1993; Brohmann et al., 1997; Cui, 2010; Hüber and Hoyer-Fender, 2007; Lim et al., 1998; Marmor and Grob-Menendez, 1991; Matzuk and Lamb, 2008; Otani et al., 1988; Oulad-Abdelghani et al., 1996; Tanaka et al., 2005). In addition, expression of Odf1 by RT-PCR was checked to confirm the correct genotyping and absence of Odf1 in homozygous Odfl-deficient mice (Fig. 3.3). I performed RT-PCR for all marker genes using cDNA generated from testes of two wild-type mice, nine heterozygous mice, and 17 homozygous Odf1-deficient mice. Besides Odf1, which was absent in homozygous Odf1-deficient mice, all marker genes were expressed in all cases, including mice that have undifferentiated or multinuclear cells present in their testis tubules (see Fig. 3.2 B). Fig. 3.3 shows the expression of Hanpl and Odf1 as an example of RT-PCR (other PCR results not shown).

Although there is normal progression of spermatogenesis up to the spermatid stage, examination of semithin sections revealed a disorganized seminiferous epithelium, and strong reduction of mature spermatozoa in caput epididymides of $O d f 1$-deficient mice (Fig. 3.4). Instead, an increase of non-mature germ cells and a high percentage 
of dysplastic sperm were found in the epididymides (Fig. 3.4).

A

$+/-$
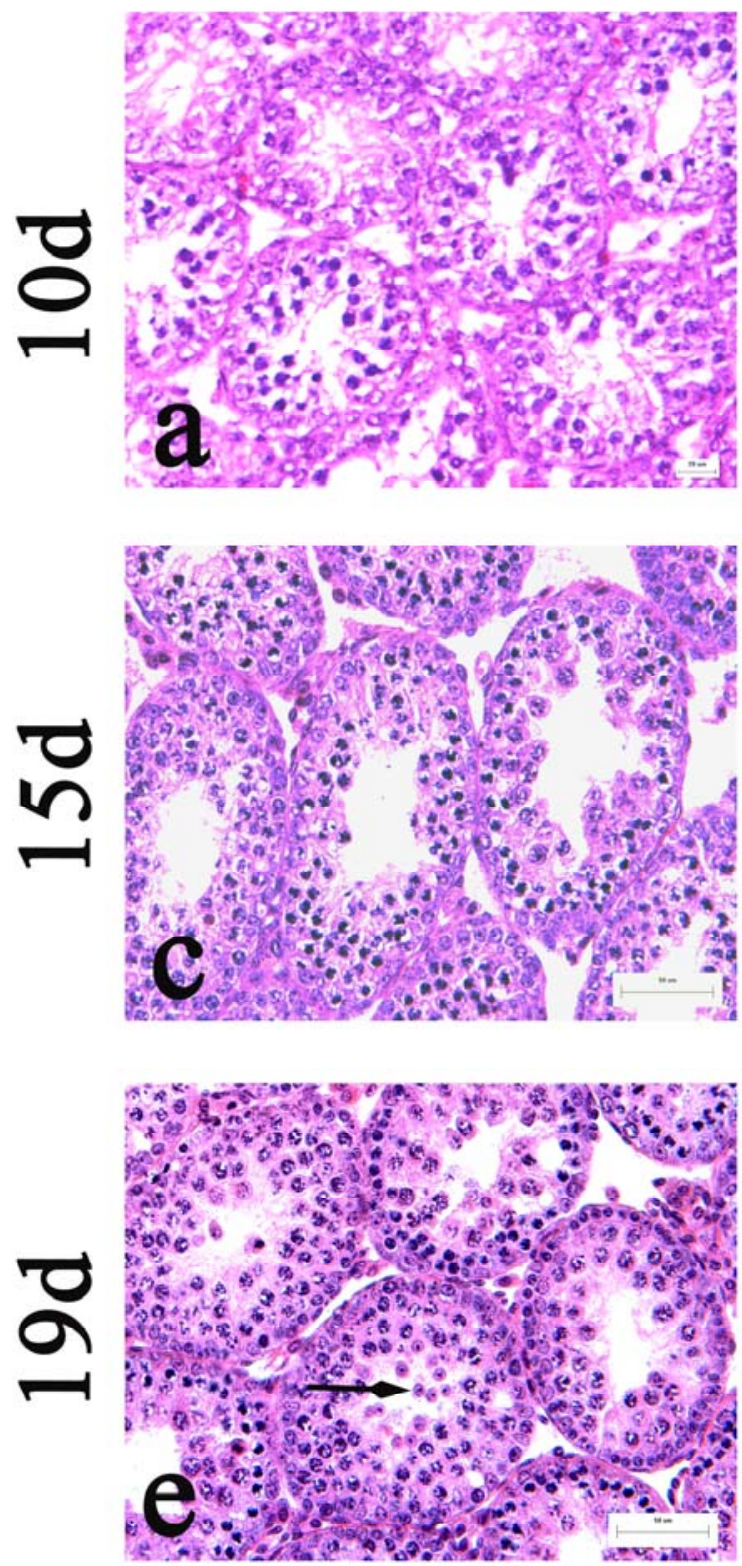

$-/-$
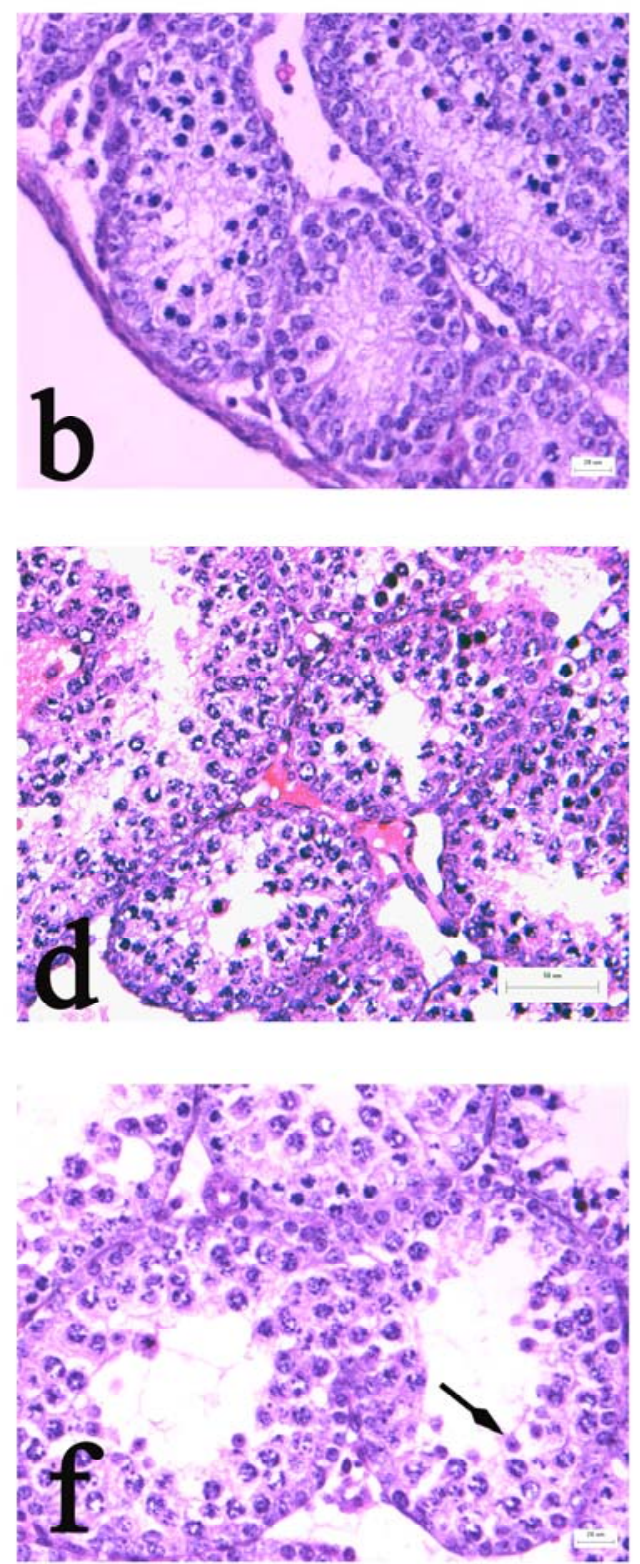


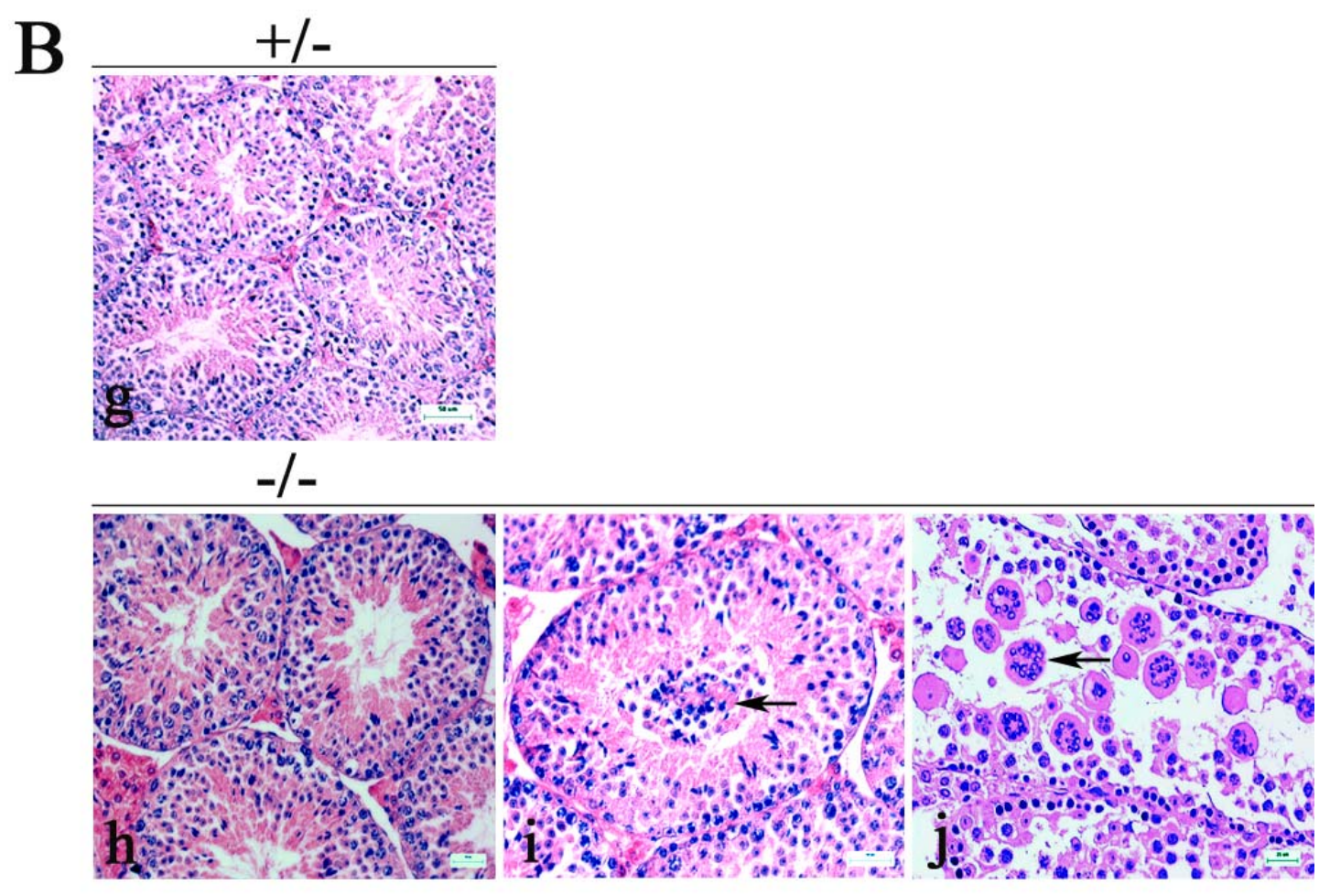

Fig. 3.2: Testicular histology of heterozygous and homozygous Odf1-deficient mice. Testis sections were stained with hematoxylin and eosin. (A), spermatogenesis in heterozygous and homozygous $O d f 1$-deficient mice. Testes from 10-day-old mice (10d), 15-day-old mice (15d), and 19-day-old mice (19d) were examined. No differences in spermatogenic progression between heterozygous and homozygous mice were observed. Round spermatids are found in $19 \mathrm{~d}$ testes of heterozygous and homozygous Odfl-deficient mice (arrows in panels e and f). For each developmental stage, the testes of one to three mice were prepared. Bars: $20 \mu \mathrm{m}(\mathrm{a}, \mathrm{b}$, and $\mathrm{f}$ ), 50 $\mu \mathrm{m}$ (c to e). (B), spermatogenesis proceeded normally in adult heterozygous mice (g). In adult homozygous mice three different phenotypes were observed. One in nine animals showed an obvious wild-type phenotype (h); six animals also had undifferentiated cells prematurely released in the lumen of the seminiferous epithelium (i; arrow), and in testes of two animals the seminiferous epithelium was flat and highly disorganized, and numerous multinuclear cells were found in the lumen ( $\mathrm{j}$; arrow). Bars: $50 \mu \mathrm{m}$ (g to i), $25 \mu \mathrm{m}(\mathrm{j})$. 


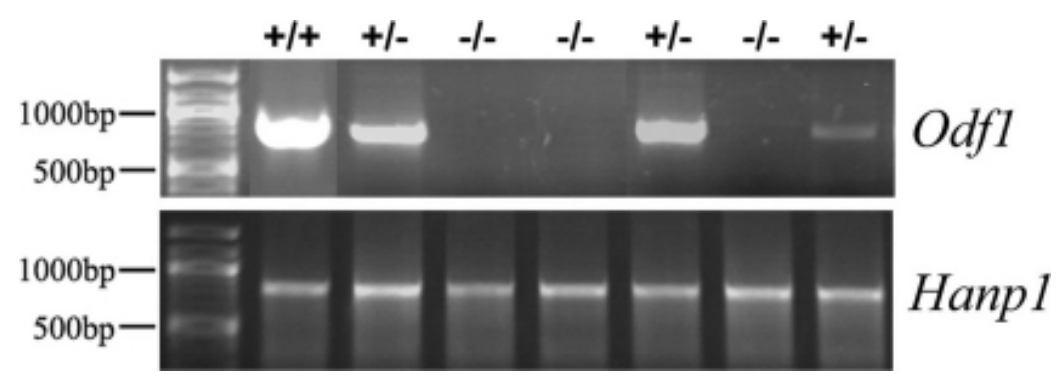

Fig. 3.3: Marker gene expression revealed spermatogenic progression irrespective of the presence or absence of ODF1. RT-PCR for Odf1 or Hanp1 was performed on cDNA generated from the testes of wild-type (+/+), heterozygous (+/-), and homozygous Odf1-deficient (-/-) mice.

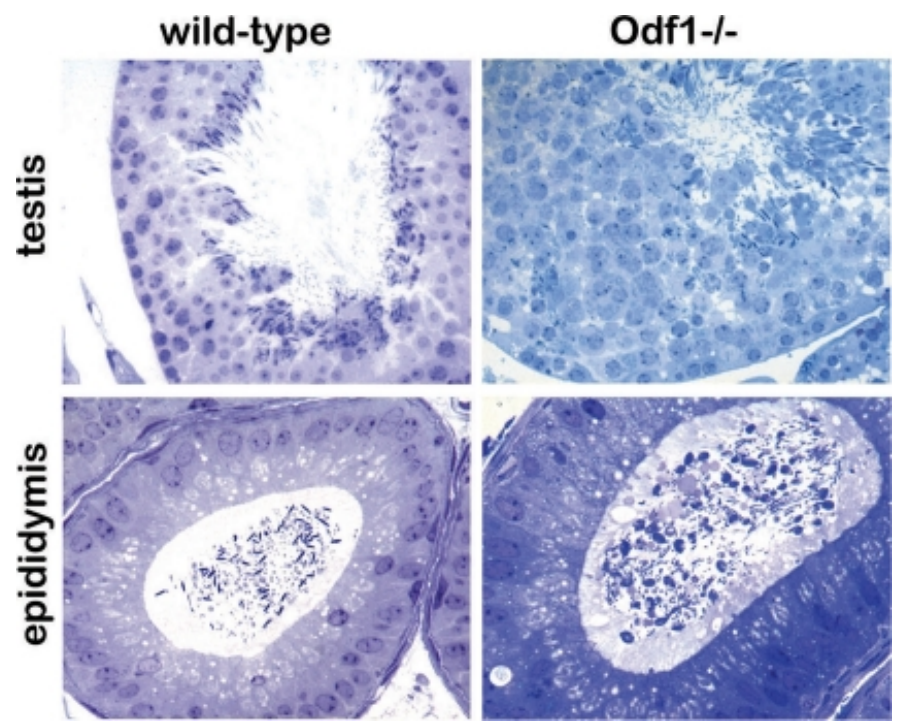

Fig.3.4: Semithin sections of wild-type and homozygous Odf1-deficient mouse testes and epididymides. Sections were stained with toluidine blue. Seminiferous tubules of wild-type and of Odf1-deficient mouse testis showed spermatogenic progression up to elongated spermatozoa and sperm tails. Sections of caput epididymides showed mature spermatozoa in the wild-type genotype, whereas in Odf1-deficient mice mature spermatozoa were barely found. Magnifications: testis, x400; epididymis, x630 (cooperation with Prof. Andreas Meinhardt, department of anatomy and cell biology, Giessen, Germany). 


\subsubsection{Sperm analyses of Odf1-deficient mice}

Histological analyses, as well as expression profiling, revealed that Odf1 deficiency did not affect the premeiotic, meiotic, and early postmeiotic phases of spermatogenesis. Even elongated spermatozoa, including the formed sperm tail, could be detected in the testes of homozygous mice. Therefore, the epididymal spermatozoa were more closely analyzed. Sperm motility was measured after capacitation in vitro, taking into consideration the sperm velocity parameters (curvilinear velocity [VCL], average path velocity [VAP], straight line velocity [VSL], and also the straightness of the movement $[\mathrm{STR}]$ ) and parameters describing the head behavior (amplitude of the lateral head displacement $[\mathrm{ALH}]$ and the beat cross frequency $[\mathrm{BCF}])$. Homozygous Odf1-deficient spermatozoa showed significantly reduced velocities (VAP, VCL, and VSL) and a reduction in lateral head displacement (ALH) compared to wild-type and heterozygous Odfl-deficient spermatozoa (Fig. 3.5). BCF and STR were significantly increased in homozygous mutants. Heterozygous Odfl-deficient spermatozoa revealed properties generally in between wild-type and homozygous spermatozoa (Fig. $3.5)$.

Then the capability of spermatozoa was analyzed to perform the acrosome reaction. Whereas in wild-type sperm as well as in heterozygous Odfl-deficient sperm a clear acrosome reaction could be monitored, homozygous sperm did not show any acrosome reaction at all and were of a conspicuously different phenotype (Fig. 3.6 A). Odfl-deficient spermatozoa were characterized by their coiling sperm tail (Fig. 3.6 A c). Immunocytology using anti- $\alpha$-tubulin antibodies revealed that the axoneme is formed in heterozygous (Fig. 3.6 B), as well as in homozygous Odfl-deficient spermatozoa (Fig. 3.6 C). PL-FITC staining showed the presence of the acrosome in heterozygous spermatozoa but not in probes of $O d f 1^{-/}$mice. Furthermore, sperm heads were generally missing in the epididymal probes of $O d f 1^{-/-}$mice (Fig. 3.6 C) 

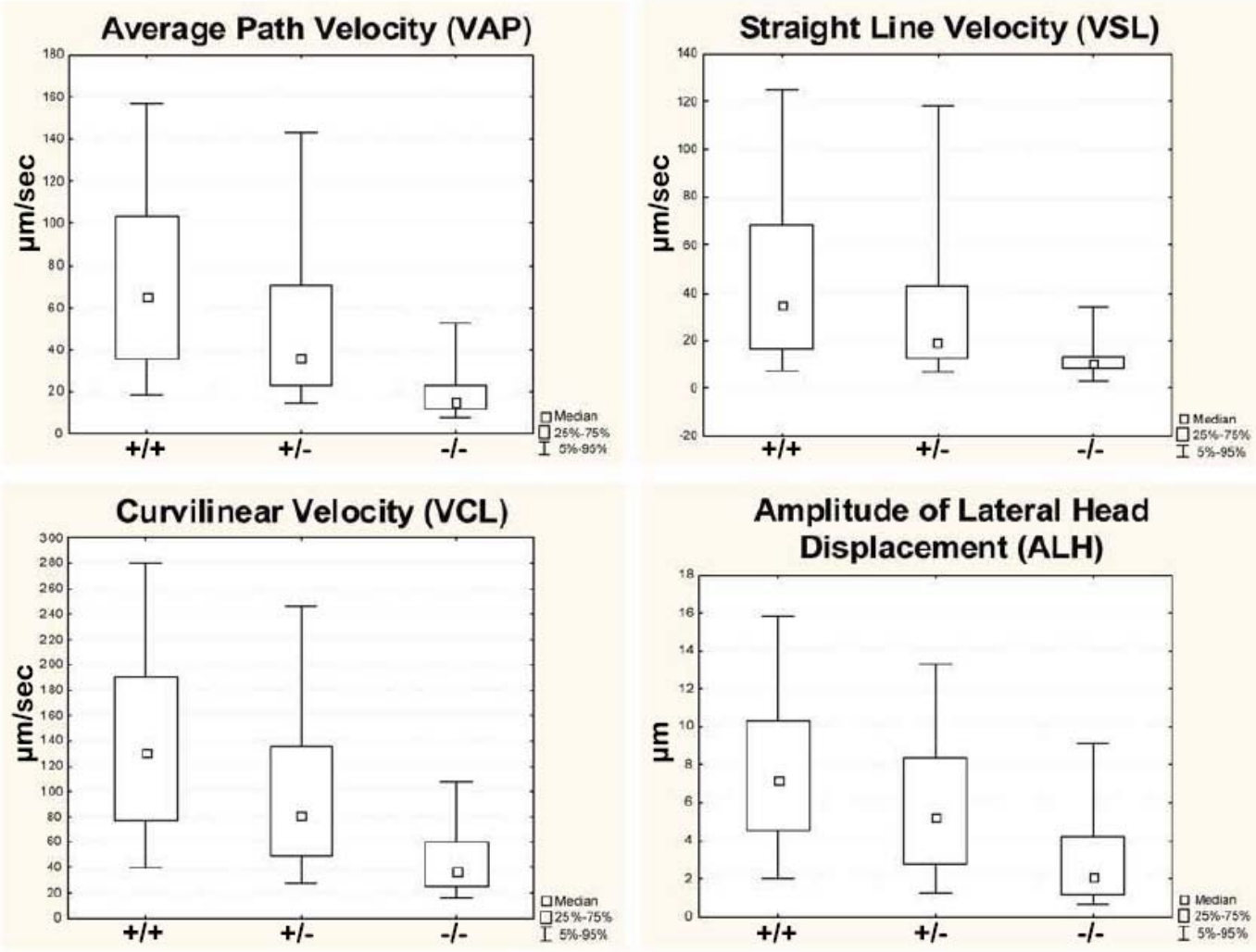

Amplitude of Lateral Head Displacement (ALH)

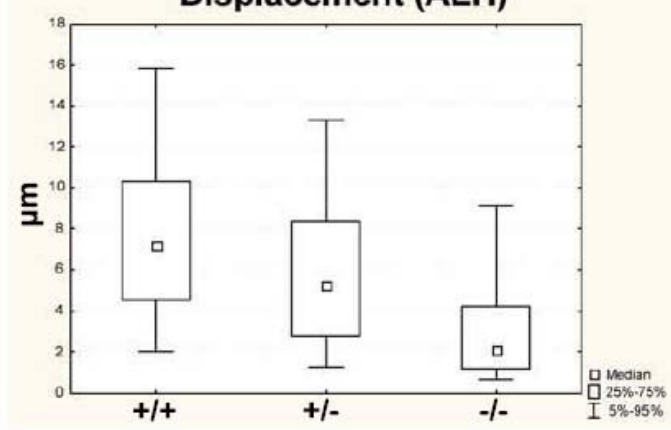

Beat Cross Frequency (BCF)

Straightness (STR)
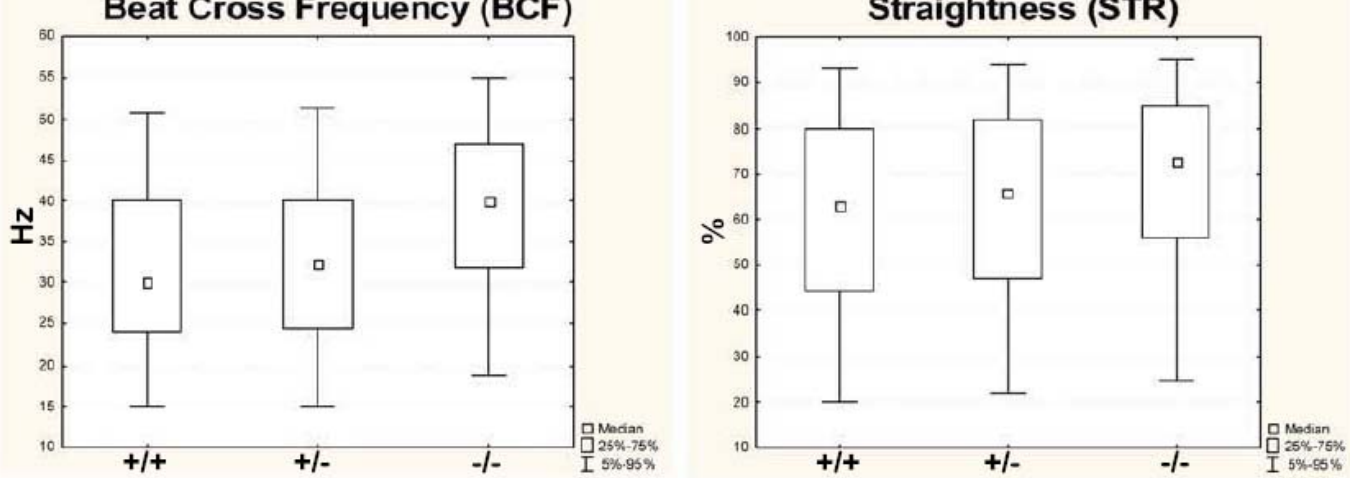

Fig. 3.5: Computer-assisted analysis of sperm motility. The parameters of wild-type $(+/+)$, heterozygous (+/-) and homozygous (-/-) Odfl-deficient spermatozoa are shown. Sperm velocities $(\mu \mathrm{m} / \mathrm{sec})$, straightness of the movement (percent), lateral amplitude of the sperm head $(\mu \mathrm{m})$, and beat frequency $(\mathrm{Hz})$ were measured. For all measurements the medians and the percentiles (25\%-75\% and 5\%-95\%) are shown. Homozygous Odf1-deficient spermatozoa exhibit significantly reduced velocities and ALH and an increased BCF and STR compared to wild-type. Velocities and amplitude of the lateral head displacement of heterozygous spermatozoa demonstrated the middle values between wild-type and homozygous spermatozoa (cooperation with Dr. Pawel Grzmil, worked at department of human Genetics, university medicine, 
Georg-August-Universität Göttingen, Göttingen, Germany; working at department of genetics and evolution, institute of zoology, Jagiellonian University, Cracow, Poland).
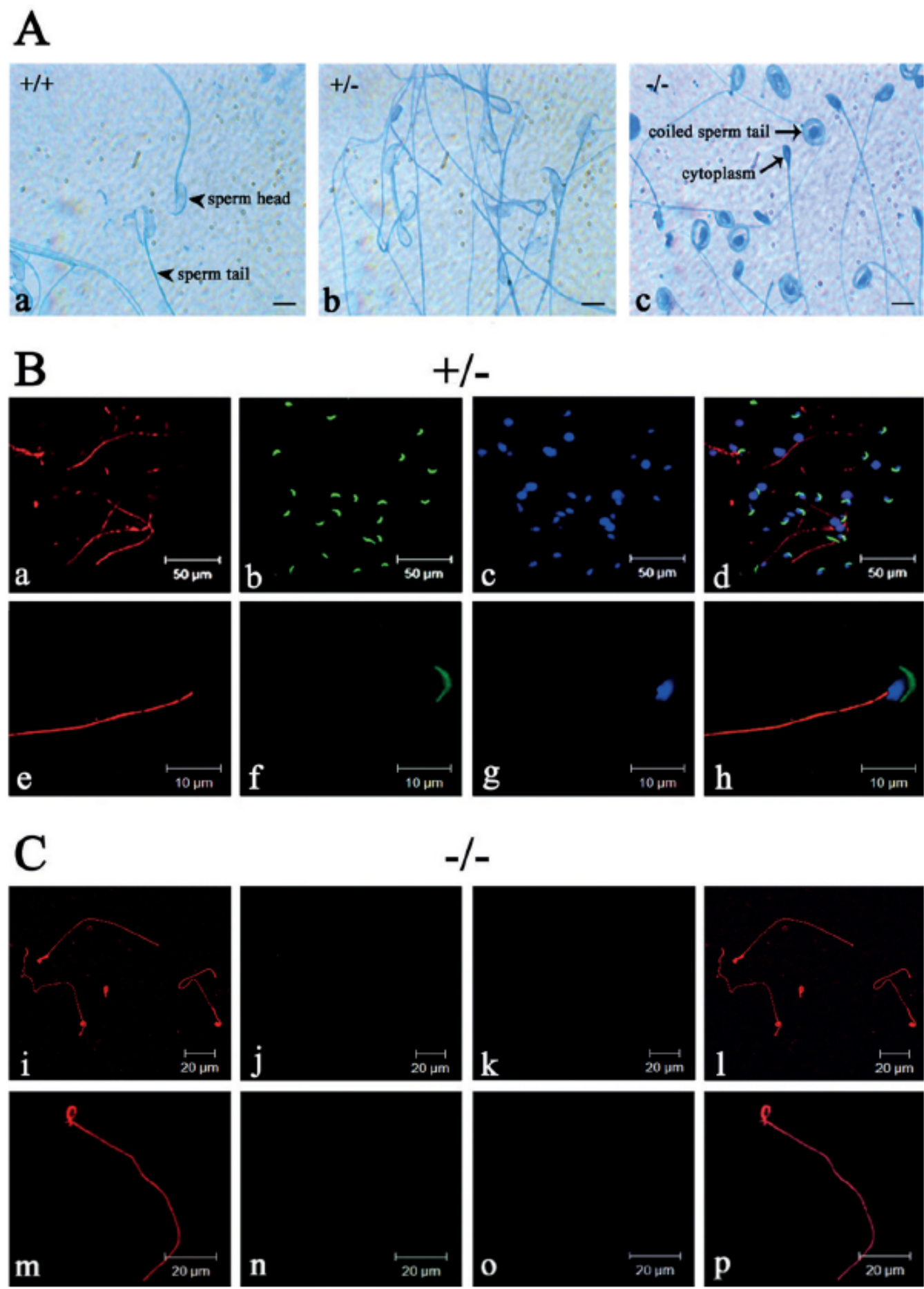

Fig. 3.6: Acrosome and axoneme in Odf1-mutant sperm. (A), acrosome reaction in epididymal sperm of wild-type $(+/+)$, heterozygous (+/-), and homozygous (-/-) mice. A characteristic light 
blue staining of the reacted acrosome is visible in wild-type $(\mathrm{a} ;+/+)$ and heterozygous $(\mathrm{b} ;+/-)$ sperm, whereas in $O d f 1^{-/-}$sperm (c; -/-) no acrosome reaction was found. In addition, a remarkable sperm coiling in $O d f 1^{-/-}$sperm is visible. Bars: $10 \mu \mathrm{m}$. (B) and (C), detection of the axonemal microtubules in heterozygous (B) and homozygous (C) Odfl-deficient spermatozoa. Sperm were released from the cauda epididymides of heterozygous $(\mathrm{B},+/-)$ and homozygous $(\mathrm{C},-/-)$ mice and stained for the acrosome with PL-FITC (in green) and for tubulin with anti- $\alpha$-tubulin antibodies (in red). Nuclear counterstain with DAPI is shown in panels $\mathrm{c}, \mathrm{g}, \mathrm{k}$, and o (in blue). In heterozygous and homozygous sperm the sperm tail was detected with anti- $\alpha$-tubulin antibodies demonstrating the presence of the axonemal structure (a, e, i, and $\mathrm{m}$ ). In homozygous sperm the tail showed a conspicuous coiling (i, $\mathrm{m}, 1$, and $\mathrm{p}$ ). The acrosome is present in heterozygous sperm ( $b$ and $f$ ) but absent in homozygous sperm $(j$ and $n$ ). In addition, characteristic DAPI-stained sperm heads are also barely visible in homozygous preparations ( $\mathrm{k}$ and o).

\subsubsection{Loss of $O d f 1$ affects the ultrastructure of the spermatozoon}

Inspection of transmission electron micrographs (EM) prepared from wild-type and homozygous $O d f 1$-deficient mice, respectively, revealed a disturbed ultrastructure in homozygous mice (Fig. 3.7 B). Most remarkably, the organization of the mitochondrial sheath in the midpiece of spermatozoa discernible in longitudinal sections and cross-sections is disturbed (Fig. 3.7 B, arrowheads). In the midpiece of wild-type spermatozoa the mitochondria are elongated, crescent-shaped organelles that are aligned end-to-end to form helices and are enclosed by a rigid capsule, the mitochondrial sheath (Otani et al., 1988). This orderly mitochondrial array is visible in longitudinal sections and in cross-sections of wild-type spermatozoa (Fig. 3.7 A, arrowheads). A disturbed mitochondrial organization is found in epididymal sperm

(Fig. 3.7 B a to c, arrowhead) as well as in testicular sections of Odf1 $1^{-/}$mice (Fig. 3.7 $\mathrm{B} \mathrm{d}$ to $\mathrm{f}$, arrowhead). In addition, the well-structured pattern of the ODFs is disturbed. ODFs are no more tightly aligned to their corresponding microtubule doublet. The disturbed structural organization of the sperm tail is evident in cross-sections of the 
midpiece when the mitochondrial sheath surrounds the sperm tail cytoskeleton (Fig. $3.7 \mathrm{~B}$ c to $\mathrm{f}$, arrows) but is also found in the principal piece of the sperm tail characterized by its surrounding fibrous sheath (Fig. 3.7 B b, arrow). In the principal piece, the ODFs are neither correctly aligned to their corresponding tubule doublets nor dislodged from them (Fig. $3.7 \mathrm{~B} \mathrm{~b}$, arrow). In addition, in the cytoplasm of a single cell more than one axonemal cross-section could be found very often, sometimes together with a longitudinal section through the axoneme, or two longitudinal sections through axonemata in parallel (Fig. $3.7 \mathrm{~B}$ a, asterisks). In contrast, in wild-type sperm tail cross-sections a highly regular pattern was found with tight association of the ODFs to their corresponding tubule doublets (Fig. 3.7 A b and c, arrow). Wild-type sperm showed a tight linkage of the sperm head to the tail and the well-known ultrastructural organization comprising basal plate, capitulum, segmented columns, and even the proximal centriole (Fig. $3.7 \mathrm{~A} \mathrm{~d}$ and e). Sperm of heterozygous mice likewise did not reveal obvious disturbances. Mitochondria, ODFs, and the connecting piece are well structured (Fig. $3.7 \mathrm{C}$ a to c). However, in ODF1-deficient epididymides no sperm heads could be found but instead only detached tails. It was thus not possible to analyze the ultrastructure of the neck region. 

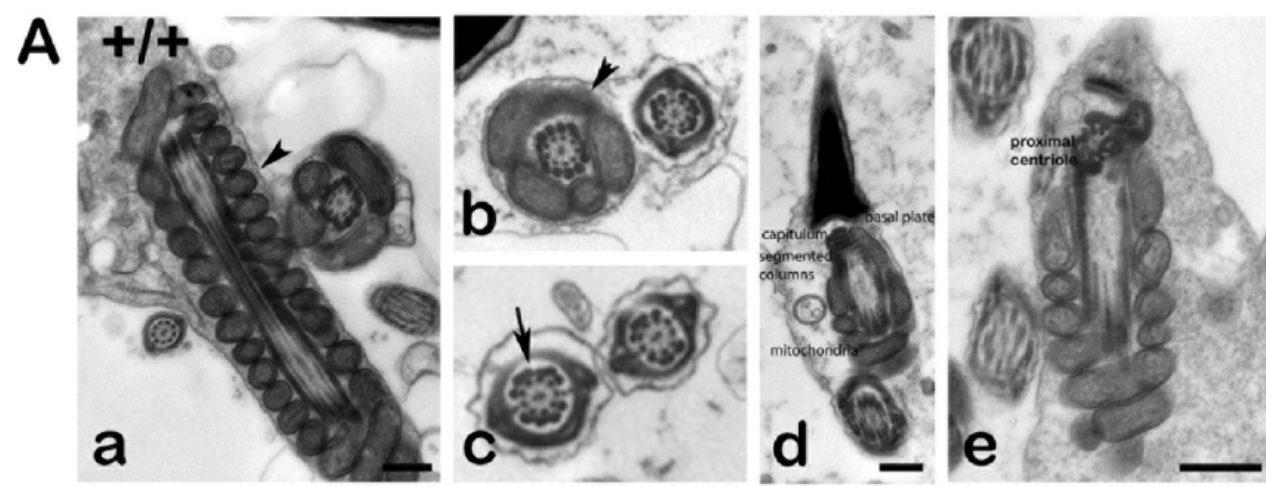

B -1
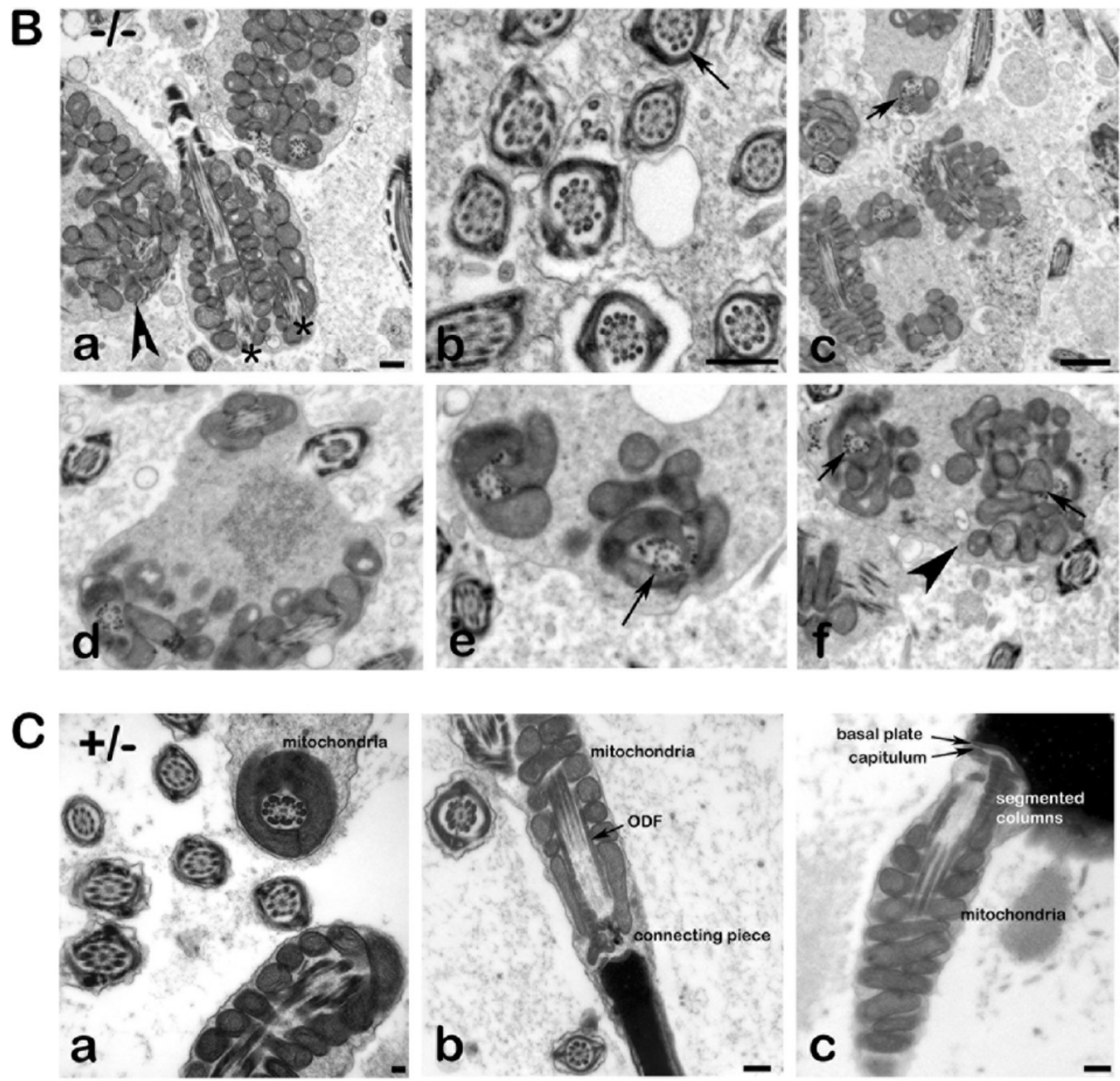

Fig. 3.7: Ultrastructure of spermatozoa. (A), transmission electron microscopy of epididymal spermatozoa from wild-type mice. The well-structured organization of the sperm is shown, including the mitochondrial sheath ( $\mathrm{a}, \mathrm{b}, \mathrm{d}$, and e, arrowheads), the outer dense fibers (c, arrow), and the neck region (d and e). Bars: $250 \mathrm{~nm}$ (a and d), $500 \mathrm{~nm}$ (e). (B), transmission electron microscopy of $O d f 1^{-/}$sperm. Epididymides (a to c) and testes (d to f) from Odf1-deficient mice 
were prepared, and sections analyzed by electron microscopy. Sperm are highly disorganized including the mitochondrial sheath ( $\mathrm{a}, \mathrm{c}$ to $\mathrm{f}$, arrowheads), as well as the outer dense fibers in the midpiece (c to $f$, arrows) and in the principal piece (b, arrow). In addition, very often in the cytoplasm of one cell more than one axonemal section was found (two longitudinal sections of axonemata in panel a, asterisks). Arrowheads, disturbed mitochondrial sheath; arrows, disturbed alignment of ODFs. Bars: 500nm (a and b), 1,000nm (c). (C), transmission electron microscopy of sperm from heterozygous mice. No obvious disturbances were found but instead well-organized mitochondria (a to c), ODFs (a and b), and connecting piece (b and c). Bars: $100 \mathrm{~nm}$ (a), $250 \mathrm{~nm}$ (b and c) (cooperation with Prof. Andreas Meinhardt, department of anatomy and cell biology, Giessen, Germany).

\subsubsection{Odf1-deficient congenic heterozygous mice (129/Sv background) have impaired male fertility}

\subsubsection{Incipient congenic heterozygous male mice are subfertile}

Odf1 deficiency on C57BL/6//129/Sv mixed background resulted in male infertility due to sperm decapitation. Additionally, Odf1 deficient sperm suffer from disorganized mitochondrial sheath and outer dense fibers. Heterozygous males are fertile but show reduced sperm motility (see 3.1.1.4 and Yang et al., 2012). In order to generate an isogenic Odf1-deficient mouse strain heterozygous Odfl-deficient mice were backcrossed with wild-type mice of strain $129 / \mathrm{Sv}$ for seven generations. I observed that litter size was dependent on the gender of the parent that carries the Odf1 knock out allele. Whereas heterozygous $O d f 1^{+/-}$-female mice from backcross generations zero to six (N0 to N6; N0 is the first mating with stain 129/Sv) gave birth to an average of 5 pups per mating (all up 40 pups in 8 litters), i.e. a litter size that is in the range of wild-type 129/Sv pairings (359 pups in 65 litters that is a mean litter size of 5.5), litter size of backcrosses using $O d f 1^{+/-}$-male mice is reduced to approximately 50\% (a total of 34 pups in 12 litters) (Fig. 3.8 A). Matings of heterozygous $\mathrm{Odf1}^{+/}$males and females of backcross generation N7 are severely 
impaired in fertility. In six matings only two pups were born after more than 5 months of continuous cohabitation. Analysing litter size during successive backcrossing revealed that fertility of female heterozygotes dropped to $\sim 3$ pups per litter in backcross generation N6 and N7 whereas male heterozygotes already have a reduced litter size in backcross generation N0. Moreover, although the fertility was impaired when heterozygous $O d f 1^{+-}$males and females of backcross generation N7 were mated, mating between heterozygous females and wild-type males of backcross generation N7 have a mean litter size of 3 pups (all in all 12 pups in 4 matings) (Fig. 3.8 B).

A

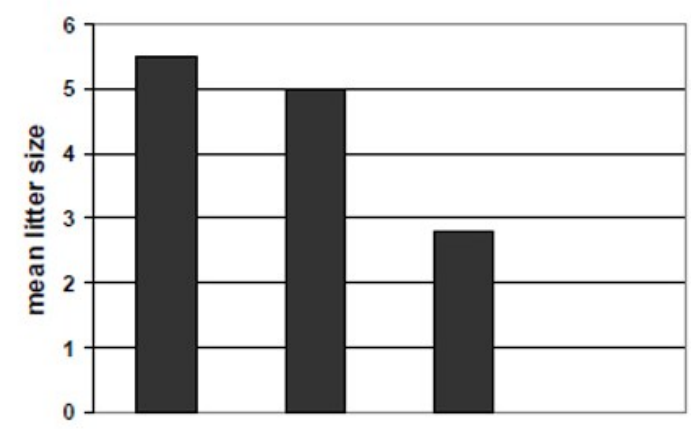

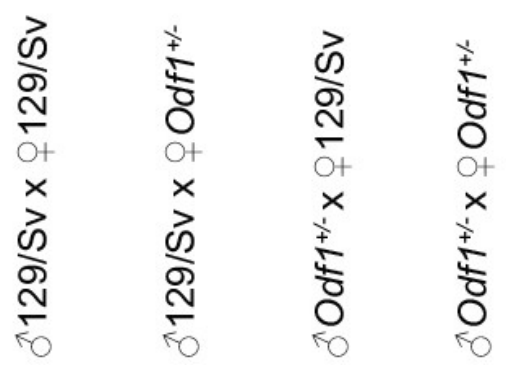

B

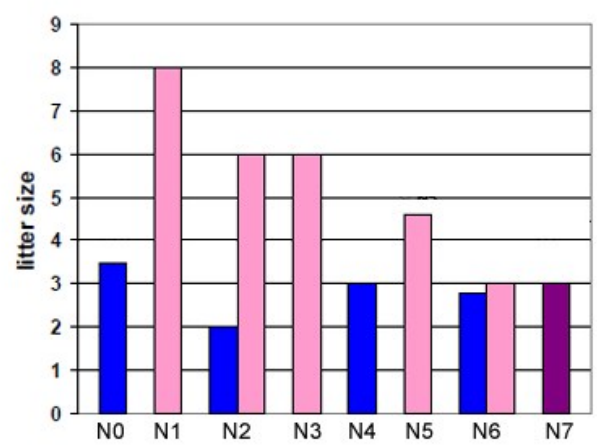

- $\delta^{+1 /-x}+129 / \mathrm{S} v$

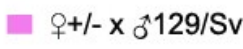

- + +l- of $\mathrm{N} 7 \times \sigma^{\star 1} 129 / \mathrm{Sv}$

Fig. 3.8: Impaired fertility of congenic heterozygous Odf1-deficient males. (A), litter size of matings on 129/Sv background. Matings of $129 / \mathrm{Sv}$ males and females gave rise to 5 pups per litter on average (all in all 359 pups in 65 litters). A similar litter size was found in matings of heterozygous Odf1-deficient females with 129/Sv males from backcross generation N0 to N6 (all up 40 pups in 8 litters). However, litter size was reduced to roughly $50 \%$ in backcrosses between $129 / \mathrm{Sv}$ females and heterozygous Odf1-deficient males (a total of 34 pups were born in 12 litters from backcross generation N0 to N6). Finally, matings between congenic heterozygous Odfl-deficient mice of backcross generation N7 are severly impaired in fertility giving rise to only 
2 pups in six matings and a mating period of 5 months. (B), male fertility is reduced in all backcross generations whereas female fertility steadily drops from 6 to 8 pups per litter (N1 to N3) to 3 pups per litter in N6 and N7. Matings of heterozygous females of generation N7 and wild-type males gave birth to approximately 3 pups per litter.

\subsubsection{Insemination capacity and sperm parameters are not altered in heterozygous males}

To figure out the underlying causes of reduced male fertility mating behaviour and sperm parameters were analysed. Heterozygous male mice $(n=6$; backcross generation N7) were mated with wild-type $129 / \mathrm{Sv}$ females and females daily inspected regarding the presence of a vaginal plug. If insemination has occurred females were killed, the liquid of the uterus obtained, and inspected for the presence of sperm. Sperm concentrations were calculated and were always in-between $5 \times 10^{6}$ to $6 \times 10^{7} / \mathrm{ml}$. As exception, two mates of one specific male did not have sperm in their uteri. Copulation behaviour and insemination capability therefore seem not to be altered in heterozygous $O d f 1^{+/-}$males. I thus wanted to know whether sperm parameters are altered. Sperms were isolated from epididymides of heterozygous $O d f 1^{+/-}$males as well as of wild-type $129 / \mathrm{Sv}$ males, respectively, after several weeks of sexual abstinence. No reduction in sperm concentration in the heterozygous condition (between 2 to $6.4 \times 10^{4}$ sperm $/ \mu 1 ; n=3$ ) compared to wild-type males (between 2.6 to $5.2 \times 10^{4} \mathrm{sperm} / \mu 1 ; \mathrm{n}=2$ ) has been found. Additionally, motility assays revealed that sperm of heterozygous males are similar to sperm of wild-type males (Fig. 3.9). 

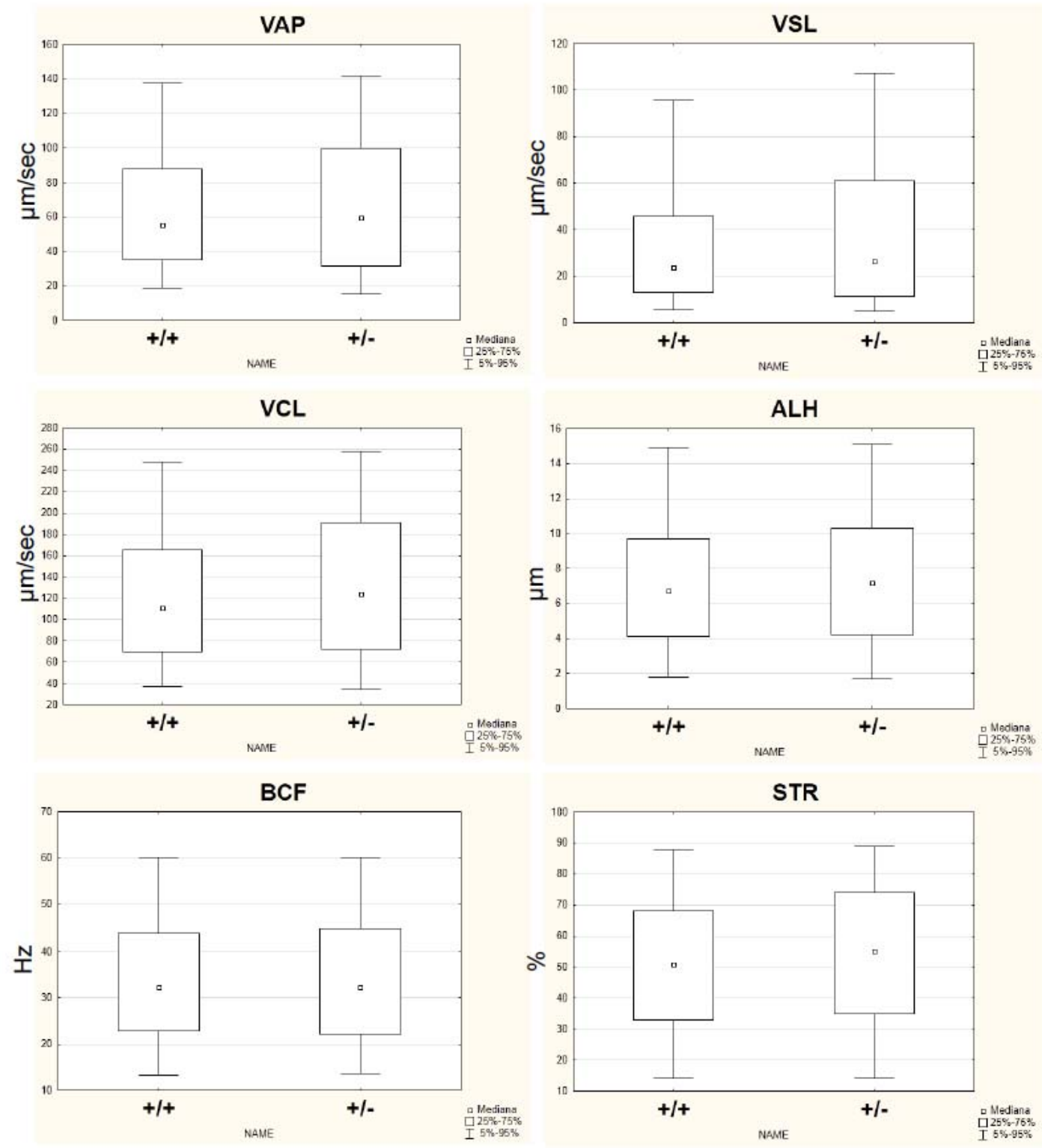

Fig. 3.9: Computer-assisted analysis of sperm motility. The parameters of wild-type (+/+) and heterozygous (+/-) Odf1-deficient spermatozoa are shown. Average path velocity (VAP), straight line velocity (VSL), and curvilinear velocity (VCL) (all in $\mu \mathrm{m} / \mathrm{sec}$ ), straightness of the movement (STR; in \%), amplitude of lateral head displacement (ALH; in $\mu \mathrm{m}$ ), and beat cross frequency (BCF; in $\mathrm{Hz})$ were measured. For all measurements the medians and the percentiles $(25 \%-75 \%$ and 5\%-95\%) are shown (cooperation with Dr. Pawel Grzmil, worked at department of human Genetics, university medicine, Georg-August-Universität Göttingen, Göttingen, Germany; working at department of genetics and evolution, institute of zoology, Jagiellonian University, Cracow, Poland). 


\subsubsection{Fertilization capacity of sperm from heterozygous $\mathrm{Odfl}^{+/-}$males is similar}

to wild-type sperm

Sperm isolated from epididymides of heterozygous $O d f 1^{+/}$males of backcross generation N7 $(n=3)$ and from one wild-type male of strain $129 / \mathrm{Sv}$ that serves as control were used for the in vitro fertilization assay. Sperm were incubated with isolated oocytes from gonadotropin stimulated, super-ovulated female mice. Incubation was performed in in-vitro-fertilization medium, and degenerated oocytes as well as 1-cell and 2-cell embryos counted 24 hours later. Wild-type sperm have fertilized more than $80 \%$ of oocytes of which $73.9 \%$ have developed to the 1 -cell stage and $11.6 \%$ to the 2 -cell stage ( $\mathrm{n}=69$ oocytes). Similarly, sperm of $O d f 1^{+/-}$males have also fertilized more than $80 \%$ of oocytes with $66.4 \%$ developed to the 1 -cell stage and $14.5 \%$ to the 2 -cell stage 24 hours later $(n=235)$ (Fig. 3.10). Thus, although heterozygous $O d f 1^{+/-}$males are severely impaired in reproduction in vitro fertilization capability is similar to those of wild-type sperm. 


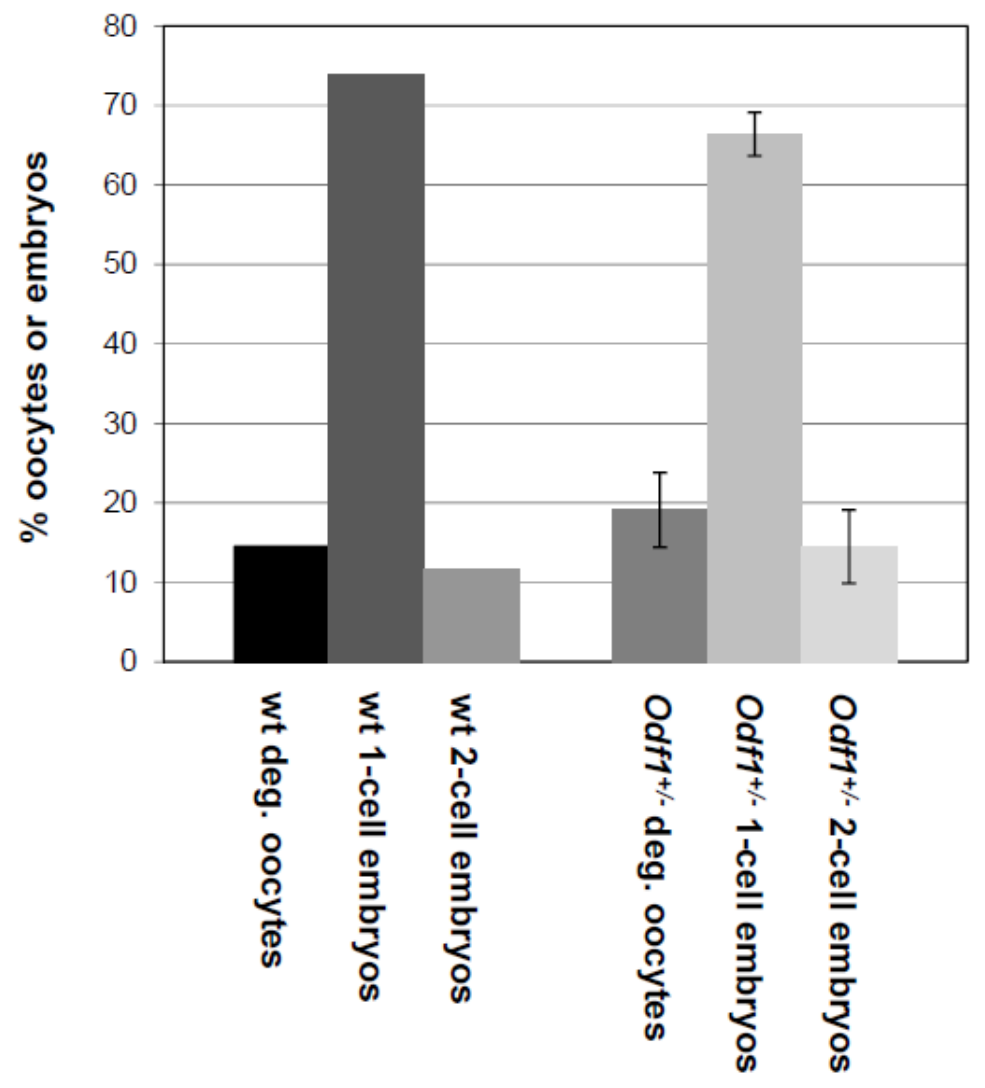

Fig. 3.10: In vitro fertilization capability of wild-type $129 / \mathrm{Sv}$ and congenic heterozygous $\boldsymbol{O d f 1}^{+/-}$sperm. Sperm were isolated from epididymides of one wild-type (wt) and three heterozygous $O d f 1^{+/}$mice and incubated with isolated oocytes from hyperovulated 129/Sv mice. 24 hours later degenerated oocytes (deg. oocytes), 1-cell and 2-cell embryos were counted. Standard deviations are included. The percentage of successful fertilization is highly similar (cooperation with Dr. Pawel Grzmil, worked at department of human Genetics, university medicine, Georg-August-Universität Göttingen, Göttingen, Germany; working at department of genetics and evolution, institute of zoology, Jagiellonian University, Cracow, Poland).

\subsubsection{Haplo-deficiency of ODF1 increased the distance between nuclear membrane and capitulum}

Odf1-deficient spermatozoa on mixed background suffer from decapitation suggesting that ODF1 is essential for the tight linkage of the spermatozoon head to the tail (see 
3.1.1 and Yang et al., 2012). I thus wanted to know whether haplo-deficiency of ODF1 on congenic background affects head to tail linkage as well therefore accounting for the observed fertility impairment. The reduced amount of ODF1 in testicular proteins of heterozygous mice was proven by Western blot analysis (Fig. 3.11 A). Electron micrographs were taken from preparations of caput and cauda epididymides as well as from testis of heterozygous mice (generation N7) (Fig. 3.11 B). A regular arrangement of mitochondria in the mid-piece of the sperm tail was observed (Fig. 3.11 B a and b), and a regular assembly of the outer dense fibers was also detected (Fig. 3.11 B b). Additionally, sperm heads are as usual connected with their tails and no structural abnormalities of the connecting piece could be found (Fig. $3.11 \mathrm{~B} \mathrm{a}, \mathrm{c}$ and d). However, more detailed analyses of the sperm-head linkage apparatus revealed that the distance between the nuclear membrane and the capitulum is 1.3 fold increased in congenic heterozygous males $(\mathrm{n}=52$ measurements) compared to that in wild-type males ( $\mathrm{n}=52$ measurements) (mean values are $85 \mathrm{~nm}$ versus $65 \mathrm{~nm}$, respectively) and that the distances are significantly different $\left(\mathrm{p}=1,77964 \times 10^{-6}\right)$ (Fig. $3.11 \mathrm{C}$ and $\mathrm{D})$. 
A
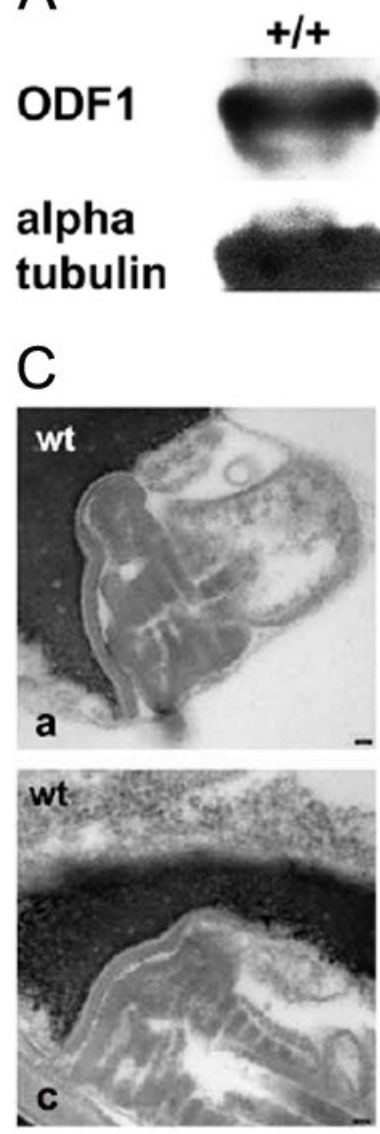
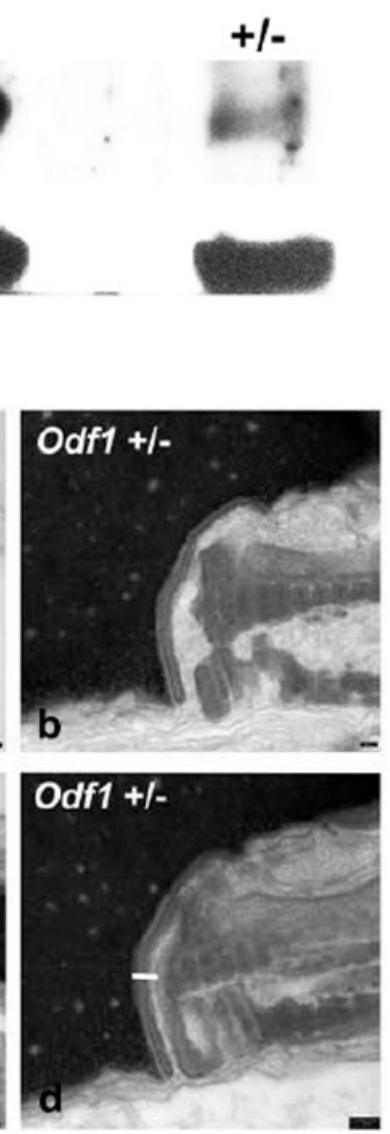

B
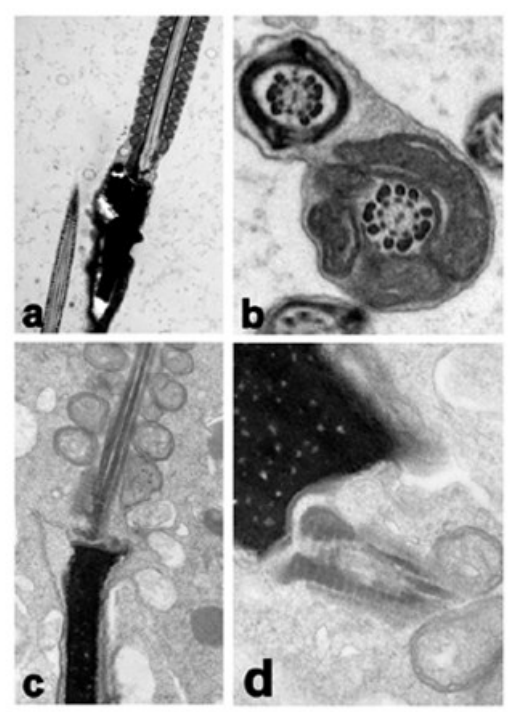

D

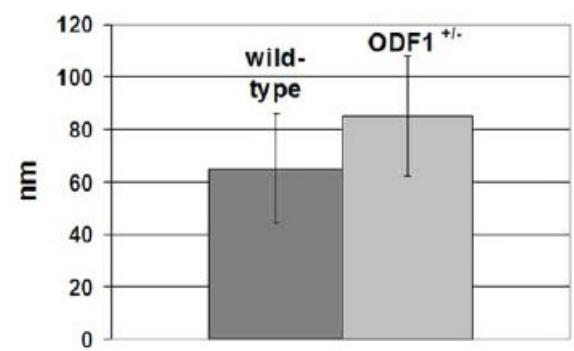

Fig. 3.11: Ultra-structural analyses of spermatozoa from incipient congenic heterozygous

$\boldsymbol{O d f 1}^{+/}$mice. (A), reduced amount of ODF1 in $\mathrm{Odfl}^{+/}$heterozygous mouse testis. Testis proteins from wild-type mice (+/+) and 129/Sv heterozygotes (+/-) were analysed for ODF1 expression by Western blot. Detection of $\alpha$-tubulin as control. (B), images were taken from probes isolated from caput (a) and cauda (b) epididymis as well as from testis (c, d). Mitochondria are regularly organized in the mid-piece region of the sperm tail $(a, b)$. The axonemal structure and the arrangement of outer dense fibers are not altered (b). Sperm heads are tightly linked to their tails with a regular formation of the connecting piece $(c, d) .(C)$, electron-micrographs were taken from the connecting piece region of wild-type (a, c; wt) and congenic heterozygous $O d f 1^{+/}$sperm (generation N7) (b, d; Odf1+/-). (D), measurements of distance between the nuclear membrane and the capitulum in sperm connecting piece. In wild-type sperm the mean distance is $65 \mathrm{~nm}$ whereas in congenic heterozygous $O d f 1^{+-}$males a mean distance of $85 \mathrm{~nm}$ was measured. Statistical analysis by Student's T-test revealed a significant difference $(\mathrm{p}<0.005)$. The approximate region used for taking measurements is marked by a white bar in d. Scale bars in electron micrographs are of $50 \mathrm{~nm}$. 


\subsection{SPAG4 protein}

\subsubsection{SPAG4 is a predicted testis specific SUN domain protein}

The full length (fl) protein consists of 443 aa with an expected molecular mass of $\sim 48$ $\mathrm{kDa}$. One alternatively spliced product was predicted encoding $747 \mathrm{bp}$ that is translated into a protein of 210 aa with a molecular mass of $\sim 23 \mathrm{kDa}$. According to Sosui and InterProScan, flSPAG4 consists of a conserved SUN domain (aa 267-427), a coiled-coil region (aa 194-251), and two transmembrane domains (aa 137-159 and 170-192). Amino acid sequence alignment of SPAG41-2 (Frohnert et al., 2011) and the putative open reading frame of SPAG4 revealed that both proteins are indeed distinct with highest similarities in the conserved SUN domain (Fig. 3.12). To investigate the expression pattern of Spag4 RT-PCR analyses were performed (Fig. 3.13). Total RNA was extracted from mouse tissues as well as from the established cell lines F9, mouse spermatogonial stem cells (SSC), and mouse embryonic stem cells (ES). RNA was digested with RNase free DNase prior to cDNA synthesis. PCR reactions were performed with primer pair Spag4 650 for/Spag4 C KpnI to amplify $\sim 800$ bp of the 3'-region of Spag4. Amplification of Gapdh was used as control. No expression of Spag4 was found in somatic tissues (heart, kidney, lungs, ovary, spleen, and epididymis; Fig. 3.13 A) whereas Spag4 is explicitly expressed in testis. To investigate Spag4 expression during spermatogenic progression, cDNA from testes of mice aged 5-dpp to 25-dpp was prepared. Spermatogenic progression is a highly ordered process that starts postnatally leading to the appearance of progressively more mature germ cells at specific times after birth (Nebel et al., 1961). Meiosis starts around day 10, early round spermatids are present approximately ten days later, and the early elongating spermatid stage is reached at about day 26 in the mouse. RT-PCR revealed that transcription of Spag4 started around day 20 to 25 postnatally and proceeded to the adult testis (Fig. 3.13 B). Expression of Spag4 therefore correlated with the formation of spermatids. Moreover, no transcription of Spag4 in embryonic stem cells (ES), in spermatogonial stem cells (SSC), and in the teratocarcinoma stem 
cell line F9 could be found (Fig. 3.13 C). Spag4 therefore seems to be specifically expressed in male germ cells. Additionally the primer pair used for amplification was verified to be specific for Spag4 and did not amplify Spag4l (Fig. 3.13 D). A clear product has been found with testis cDNA as template whereas no product was generated using Spag4l cDNA as template.

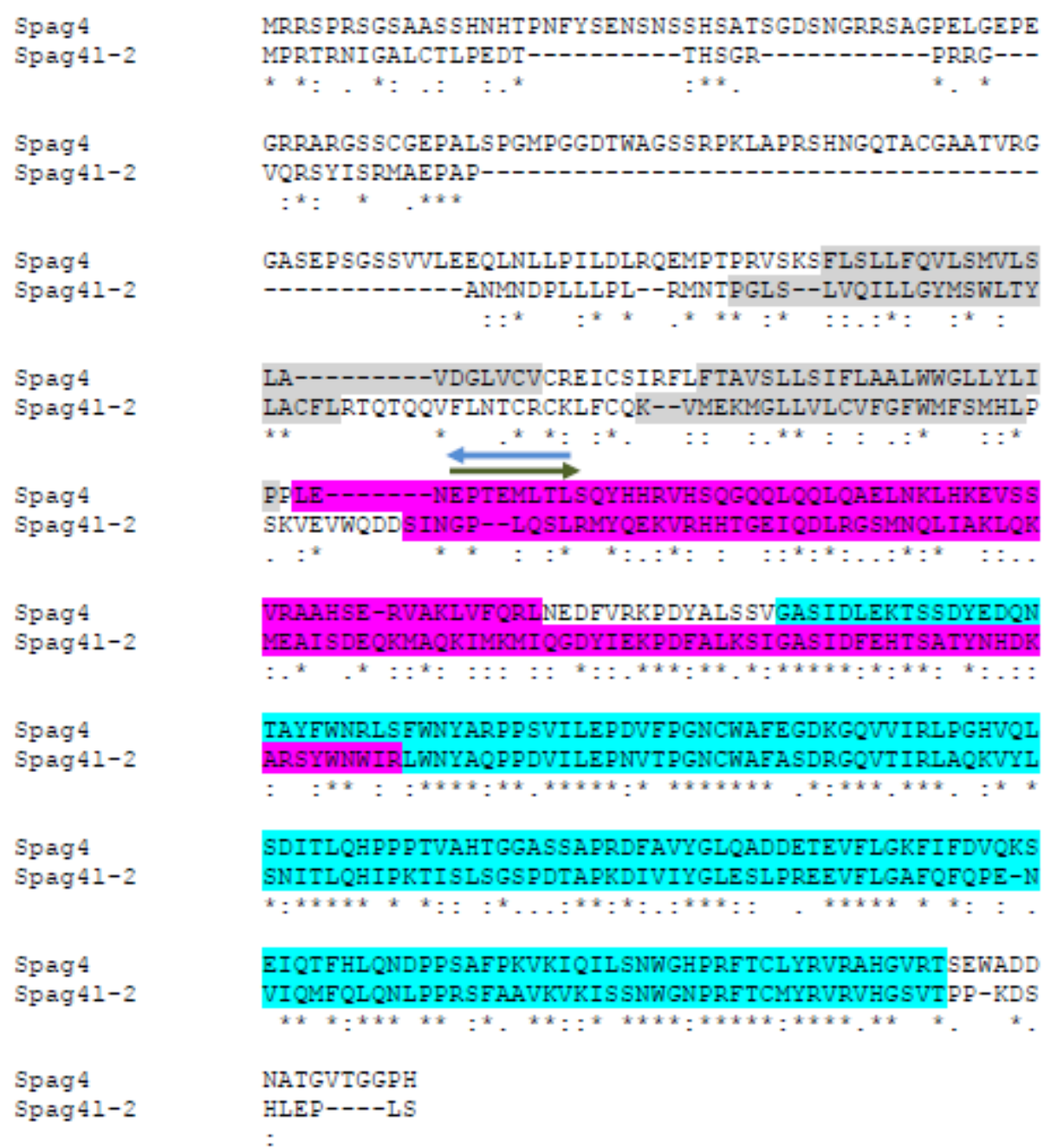

Fig. 3.12: Amino acid sequence alignment and domain organization of SPAG4 and

SPAG4L-2 from Mus musculus. Both proteins showed a similar structural organization with two putative transmembrane domains (in grey), one coiled-coil domain (in pink), and the SUN domain (in blue). Despite their related nomenclature, SPAG4 and SPAG4L are distinct proteins. SPAG4 encoding the N-terminal part (SPAG4-N) stops at aa MLTL (blue arrow), SPAG4 encoding the C-terminal part (SPAG4-C) starts with aa EPTE (green arrow). 

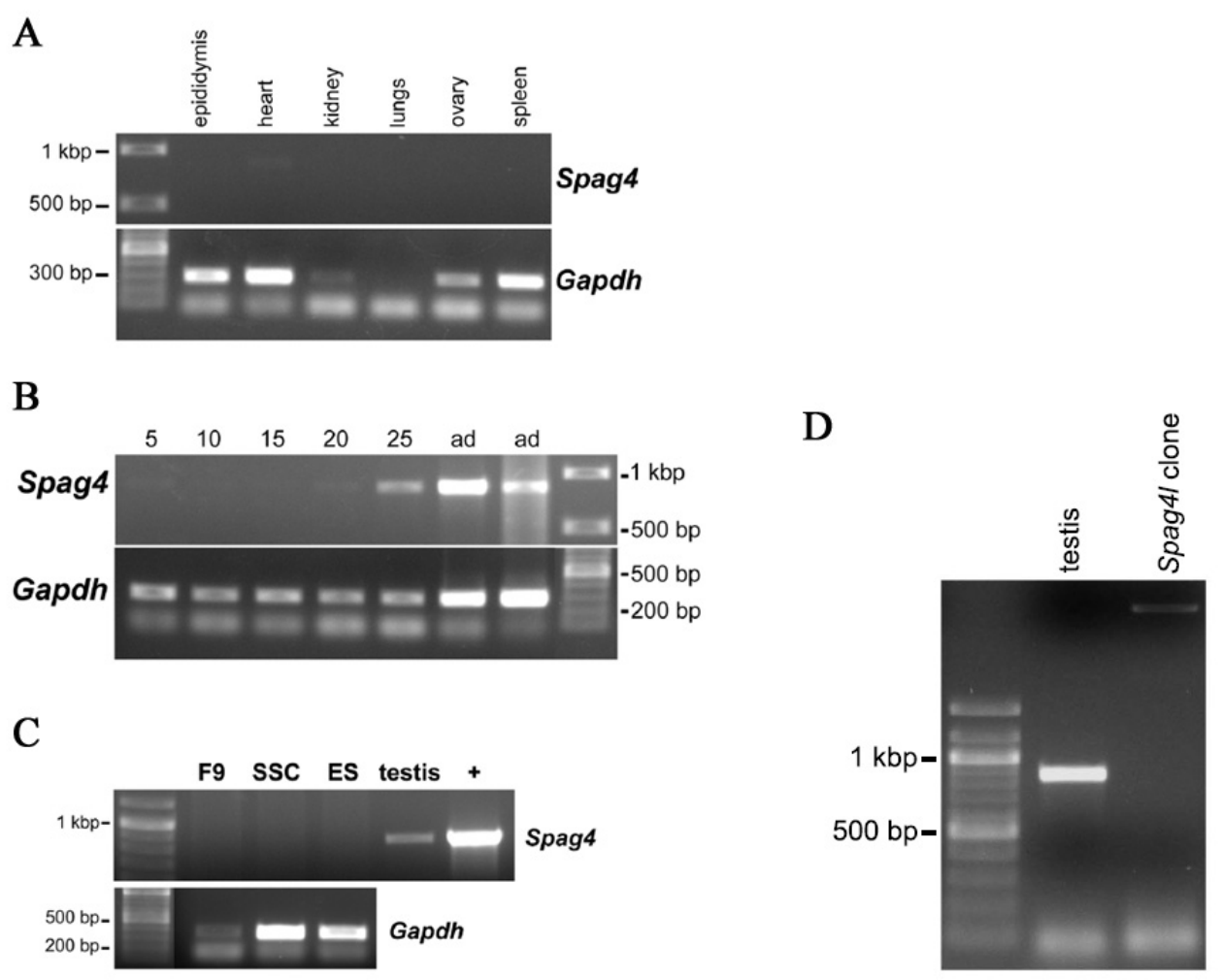

Fig. 3.13: Spag4 is specifically transcribed in male germ cells. RT-PCR was performed to amplify 800bp of Spag4, and Gapdh as control. (A), Spag4 is not transcribed in somatic tissues. (B), Spag4 is transcribed in testis starting around day 20 after birth. Transcription lasts to adult testis (ad). (C), Spag4 is not transcribed in ES, SSC, and F9 cells. As positive controls, testis cDNA (testis), and Spag4 cDNA clone (+) were used. Amplification of Gapdh was always used as control for the integrity of cDNAs. (D), specificity of amplification of Spag4 but not Spag4l. Amplification with primer pair Spag4 650 for/Spag4 C KpnI was performed on testis cDNA (testis) as well as on Spag $4 l \mathrm{cDNA}$ clone.

\subsubsection{The over-expressed SPAG4 in somatic cells locates to nuclear membrane}

Full length Spag4 cDNA was cloned into pcDNA3.1 to yield a protein with the HA-tag at its N-terminal end and the Myc-tag at its C-terminal end. The plasmid was transfected into HEK293 cells and the fusion protein detected with anti-HA and 
anti-Myc antibodies, respectively. In either case, HA-SPAG4-Myc was detected at the nuclear membrane (Fig. 3.14). Furthermore, HA-SPAG4-Myc located also to the cytoplasm probably due to its abundant over-expression in the somatic cell line. Additionally, anti SPAG4 antibody specifically detected HA-SPAG4-Myc at the nuclear membrane whereas HA-SPAG4L2-Myc (Frohnert et al., 2011) was not detected thus confirming the specificity of the antibody. The full length SPAG4 protein therefore is located at the nuclear membrane.

All SUN domain proteins characterized so far are transmembrane proteins spanning the inner nuclear membrane (INM) with their N-terminal region oriented towards the nucleoplasm and the C-terminally located SUN domain positioned in the luminal space between outer nuclear membrane (ONM) and inner nuclear membrane (INM). To further substantiate the topology of SPAG4, pHA-Spag4-Myc was transfected into NIH3T3 cells and in situ digested with proteinase K (Frohnert et al., 2011). Cells were either incubated with proteinase K in KHM buffer (Fig. $3.15 \mathrm{~S} 1$, P1), or with proteinase $\mathrm{K}$ in KHM buffer containing Triton X-100 (Fig. 3.15 S2, P2). Cells incubated in buffer without Triton X-100 might be largely inaccessible for proteinase $\mathrm{K}$ treatment whereas Triton $\mathrm{X}-100$ rendered the cells permeable thus enabling proteolytic digestion. Proteinase K digestion in the presence of Triton X-100 resulted in a nearly complete degradation of proteins. Only a very faint band of SPAG4 at $\sim 40$ kDa could be detected with anti-Myc antibodies but not with anti HA-antibodies (Fig. $3.15, \mathrm{P} 2)$ suggesting that proteolytic degradation started from the $\mathrm{N}$-terminal end with the HA-tag. A C-terminal fragment of about $40 \mathrm{kDa}$ is expected when the $\mathrm{N}$-terminal part up to the transmembrane domain is degraded. However, proteolytic degradation took also place in the absence of Triton X-100. Besides full length SPAG4 of $>55$ $\mathrm{kDa}$, several degradation products were detected with anti-Myc antibodies as well as with anti-HA antibodies (Fig. 3.15, S1, P1). The full length SPAG4 is shown in the control (Fig. 3.15, C). Since the C-terminal end of SPAG4 was tagged with Myc and the N-terminal end with HA, C-terminal or N-terminal degradation products could specifically be identified with anti-HA or anti-Myc, respectively. Anti-myc antibodies clearly detected the full length protein of $>55 \mathrm{kDa}$ in the supernatant (S1) as well as 
in the pellet (P1) along with smaller proteolytic fragments suggesting that degradation started from the N-terminal end leaving the C-terminal myc-tag sheltered. Using anti-HA antibodies, proteolytic fragments were detected as well, with a protein fragment of $\sim 40 \mathrm{kDa}$ as the largest fragment whereas the full length protein was no longer present. These fragments contain the N-terminal HA-tag and are therefore derived from successive C-terminal digestion. Strong proteolytic degradation probably resulted from liberated proteins caused by experimentally induced destruction of cells. Therefore, proteolytic fragments might also include C-terminally Myc-tagged and N-terminally HA-tagged fragments. These results suggest that the $\mathrm{N}$-terminal HA-tag is more accessible to proteinase $\mathrm{K}$ digestion than the C-terminal Myc-tag. The flSPAG4 protein therefore seems to have a similar orientation within the nuclear membrane as other SUN domain proteins with the C-terminal end located in the perinuclear space. Due to experimental conditions of ectopic over-expression of SPAG4 a conspicuous amount of SPAG4 is also degraded from the C-terminal end but leaves the N-terminal HA-tag intact (see also Fig. 3.14).

Dimerization of SUN domain proteins has been described in the literature. I therefore wanted to know whether SPAG4 is also able to form homodimers. HEK293 cells were co-transfected with EYFP-SPAG4-Myc and HA-SPAG4-Myc expression plasmids thus enabling detection of two different SPAG4 molecules individually. Cells were lysed and soluble proteins were subjected to immunoprecipitation using anti-GFP antibodies which were raised in rabbit. The bead bound protein fraction was then analyzed by Western blot using monoclonal anti-HA antibodies. HA-tagged SPAG4 (at $\sim 55 \mathrm{kDa}$ ) was detected not only in the soluble protein fraction and the protein pellet (Fig. 3.16, lanes $\mathrm{s}$ and $\mathrm{p}$, respectively) but was also found to co-precipitate with SPAG4-EYFP fusion protein (Fig. 3.16 lane b). In contrast, no SPAG4 was found in the control beads in which anti-GFP antibodies have been omitted (Fig. 3.16, lane bc). These results thus demonstrate that SPAG4 forms homodimers. 

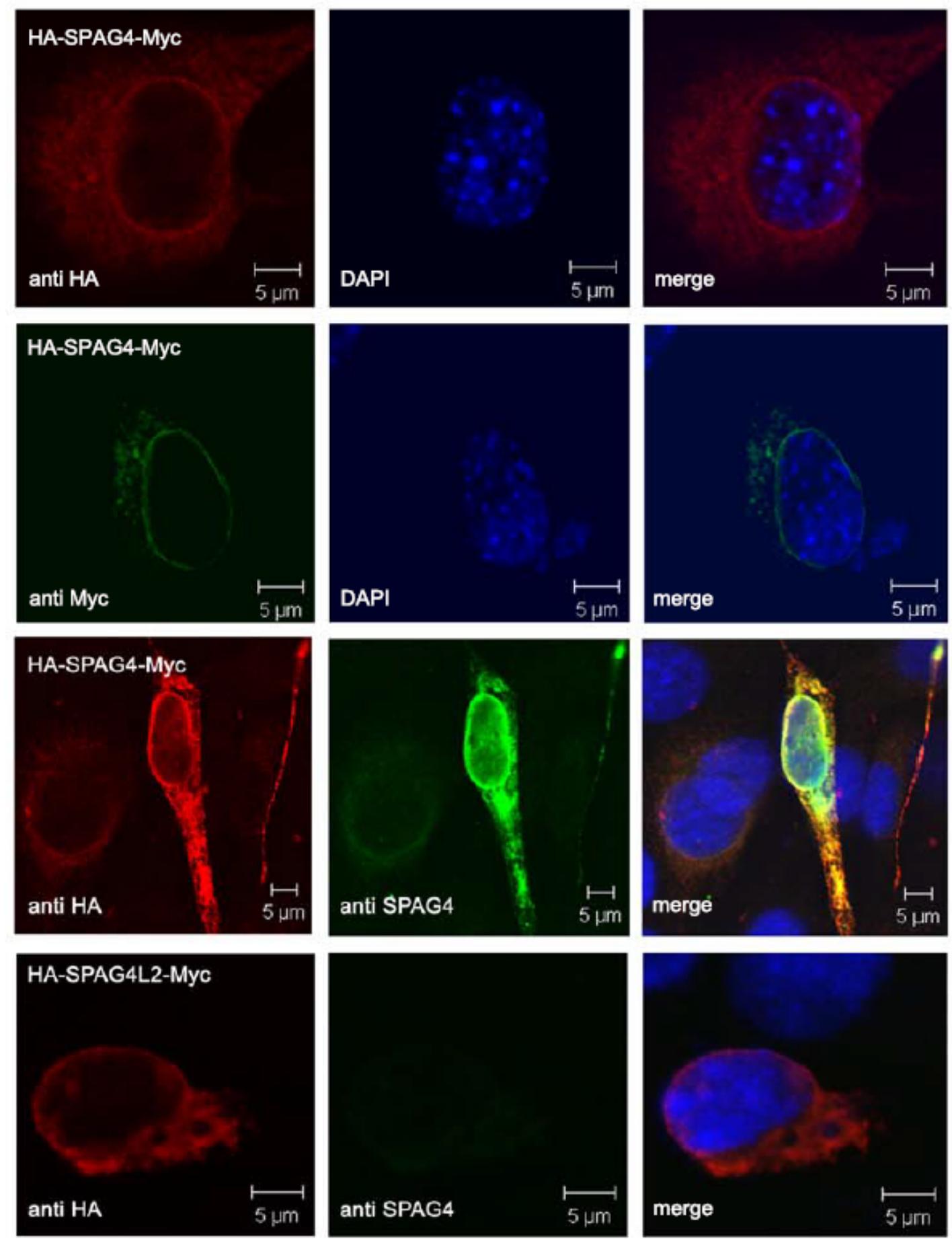

Fig. 3.14: Ectopic expression of SPAG4 in HEK293 cells revealed nuclear membrane localization. A plasmid encoding SPAG4 tagged with HA at its N-terminal end and Myc at its C-terminal end was transfected into HEK293 cells and detected with anti-HA (in red), anti-Myc (in green), and anti SPAG4 antibodies (in green), respectively. Anti SPAG4 antibody specifically detected SPAG4 but did not react with transfected SPAG4L2 (tagged with HA at its N-terminal 
end and with Myc at its C-terminal end; HA-SPAG4L2-Myc) although expression of HA-SPAG4L2-Myc was proven by anti-HA antibodies (in red). Nuclear counterstaining with DAPI in blue.

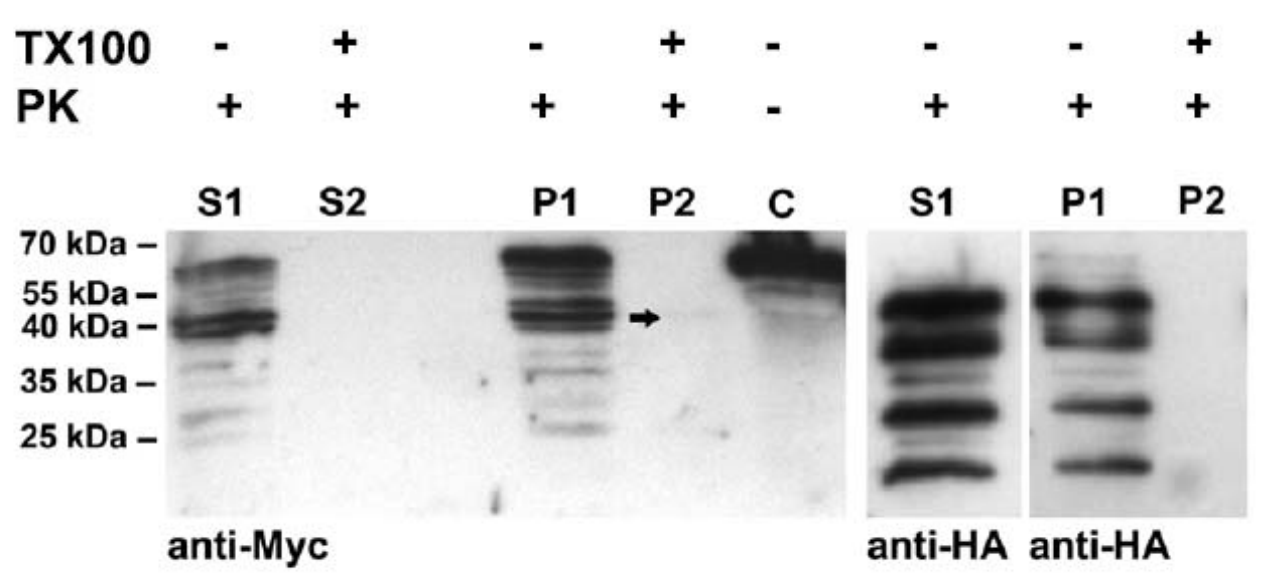

Fig. 3.15: The N-terminal end of SPAG4 is prone to in situ proteinase $\mathrm{K}$ digestion. pHA-Spag4-Myc was transfected into NIH3T3 cells and subjected to in situ proteinase K digestion. Scraped cells were exposed to proteinase K either in the absence (S1, P1) or in the presence of Triton X-100 (S2, P2). Proteins were separated on denaturing SDS-gels, blotted onto nitrocellulose membranes, and probed with the indicated antibodies anti-Myc or anti-HA. The full length SPAG4 protein is shown in the control (C). Proteinase $\mathrm{K}$ digestion started from the $\mathrm{N}$-terminal end tagged with HA since no full length protein could be detected with anti-HA antibodies. In contrast, anti-Myc antibodies detected the full length protein of $\sim 70 \mathrm{kDa}$. In P2 a very faint band of about $40 \mathrm{kDa}$ could be detected with anti-Myc antibodies but not with anti-HA antibodies (arrow). Presence (+) or absence (-) of proteinase K (PK) or Triton-X100 (TX100) are indicated (This experiment was performed by Prof. Dr. Sigrid Hoyer-Fender). 


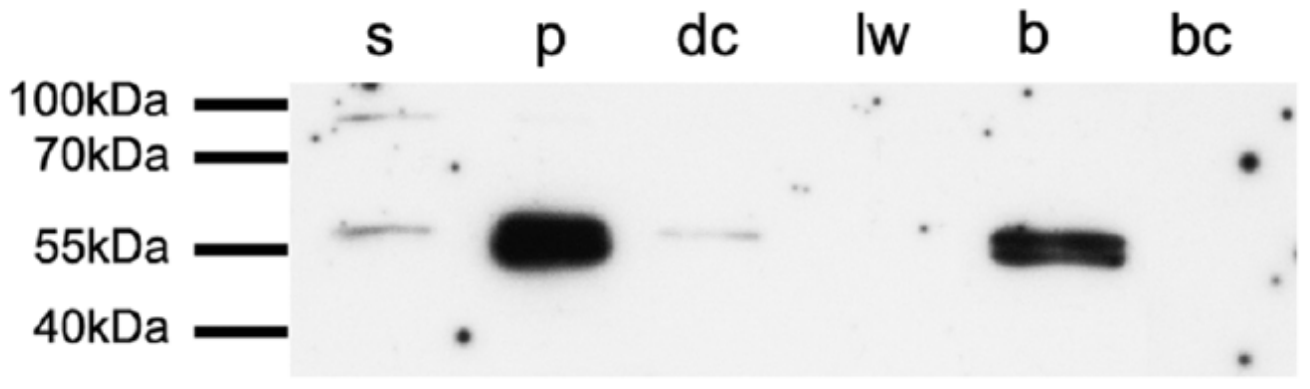

Fig. 3.16: Confirmation of SPAG4 self-interaction by co-immunoprecipitation. HEK293 cells were co-transfected with EYFP-SPAG4-Myc and HA-SPAG4-Myc expression plasmids. After cell lysis soluble proteins were subjected to immunoprecipitation using anti-GFP antibodies (raised in rabbit). Bead bound proteins were then dissolved in denaturation buffer and separated on SDS-polyacrylamide gels. After Western blot target proteins were detected with anti-HA antibodies (raised in mouse) at $\sim 55 \mathrm{kDa}$. There are two closely standing bands on lane $\mathrm{b}$. They are supposed to be the complete fusioned HA-SPAG4-MYC protein and the main part HA-SPAG4 lost its Myc-tag. s, supernatant of cell lysate; p, pellet, insoluble cellular fraction; dc, depletion control, proteins of the supernatants after separation of protein $G$ agarose beads; lw, last wash; $b$, proteins bound to beads after incubation with fishing antibody; bc, control, proteins bound to beads without the presence of the fishing antibody.

\subsubsection{The distribution of endogenous SPAG4 in male germ cells during spermiogenesis}

From 3.2.1 and 3.2.2 it is known that SPAG4 is a novel SUN domain protein located in the nuclear membrane and restricted to spermatids. Subsequently, the next question was, do the endogenous SPAG4 proteins also associate with the nuclear membrane of male germ cells? Fig. 3.17 shows that the protein revealed dynamic distribution during spermiogenesis albeit always associated with the nuclear membrane. In early round spermatids SPAG4 is first scattered over the nuclear membrane and then became more and more restricted towards the distal pole where the transient manschette is forming (Fig. 3.17 A and B). In elongating spermatids SPAG4 
distribution is similar to the location of the manchette (Fig. $3.17 \mathrm{C}$ and D). In later spermatid stages the manchette gradually disintegrated and SPAG4 became concentrated towards the distal pole at a position where the sperm tail inserts (Fig. 3.17 E-G).

\section{alpha tubulin SPAG4 DAPI}
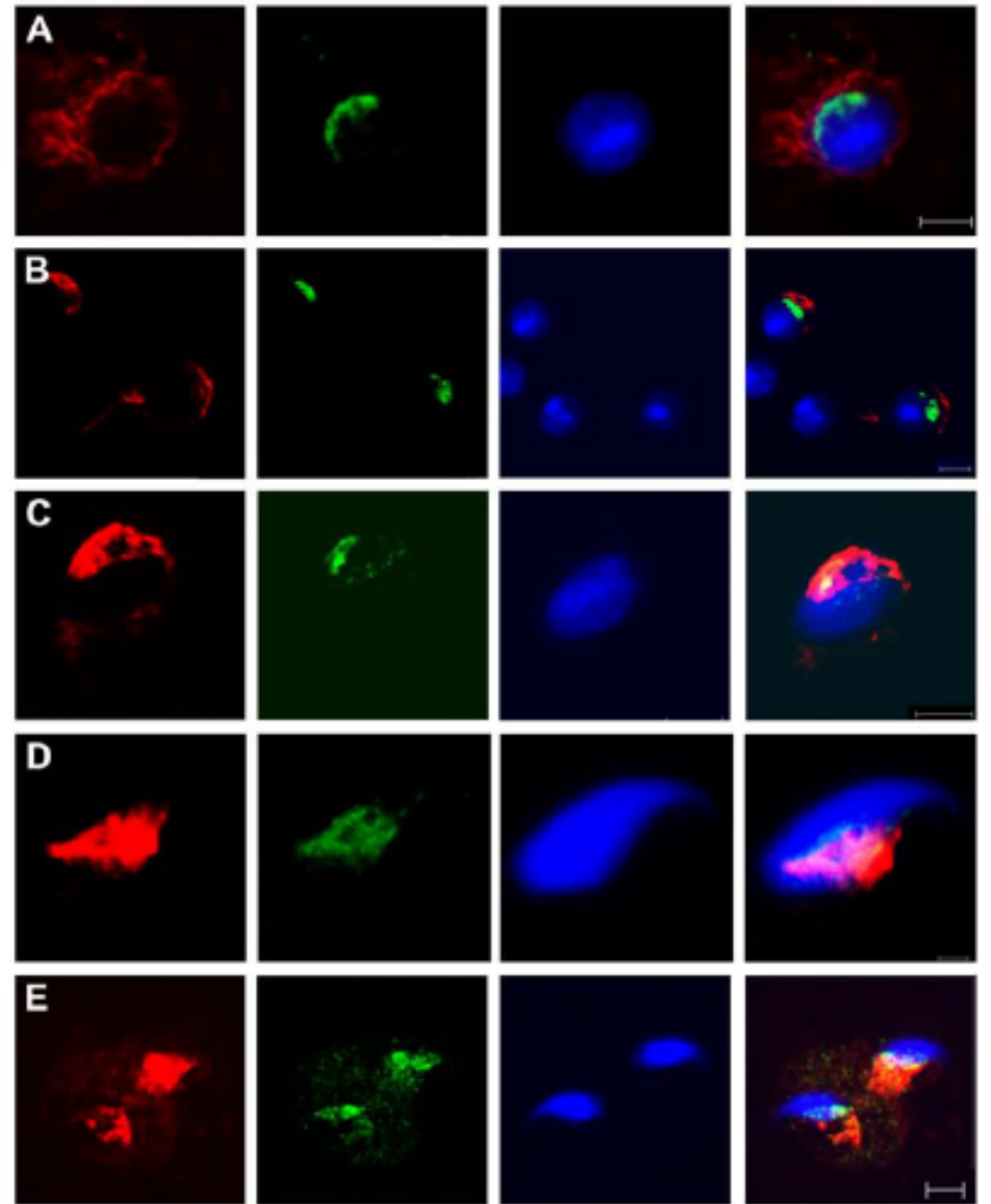

$\mathbf{F}$
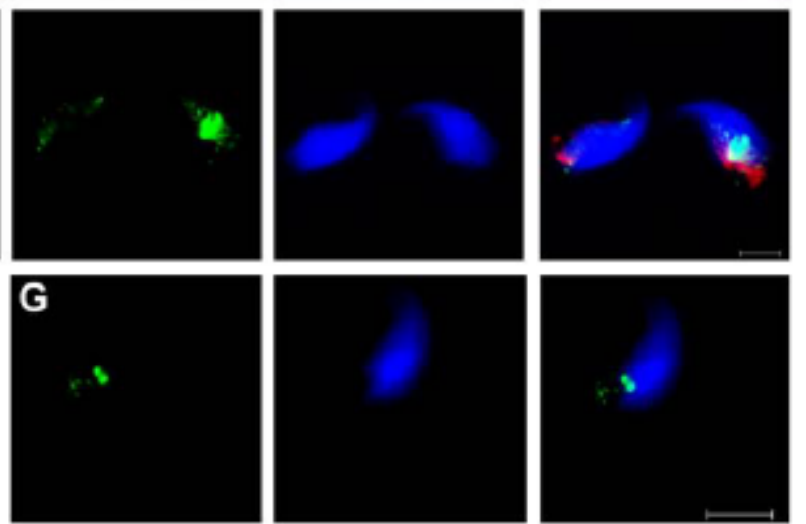
Fig. 3.17: Endogenous expression of SPAG4 in spermatids. Suspension preparations from mouse testis were incubated with anti-SPAG4 antibodies (green) and anti- $\alpha$ tubulin antibodies (red). SPAG4 is first scattered over the round spermatid nucleus (A) and later on became restricted towards the posterior pole where the manchette is forming (B). In elongating spermatids SPAG4 is associated with the manchette $(C, D)$. In later spermatid stages the manchette disintegrated and SPAG4 became concentrated at the distal pole (E-G). DAPI counterstain in blue. Scale bars in A, B, C, E, F, G: $5 \mu \mathrm{m}$, in D: $2 \mu \mathrm{m}$.

\subsubsection{SPAG4 interacts with ODF1}

SPAG4 (sperm associated antigen 4) was originally identified as a sperm specific protein that interacts with the sperm tail outer dense fiber protein ODF1 without being incorporated into the sperm tail (Shao et al., 1999). Due to its wide expression in human neoplastic tissues SPAG4 was described moreover as a potential cancer marker (Kennedy et al., 2004). SPAG4/ODF1 interaction thus first is verified by co-immunoprecipitation out of mouse testis. Endogenous SPAG4 was precipitated with goat anti-SPAG4 antibodies and endogenous ODF1 detected by Western blot using rabbit anti-ODF1 antibodies. ODF1 (at $\sim 35 \mathrm{kDa}$ ) was detected in the beads bound protein fraction (Fig. 3.18 A, lane b). Only a very faint signal was also found in the beads only control fraction (Fig. $3.18 \mathrm{~A}$, lane bc) thus demonstrating interaction between SPAG4 and ODF1. Specificity was further proven using testicular proteins from $O d f 1^{-/}$mice for co-IP (see 3.1 and Yang et al., 2012). SPAG4 was precipitated first and Western blots incubated with anti-ODF1 antibodies (Fig. 3.18 B). As expected, no ODF1 could be found. Additionally, the $\sim 35 \mathrm{kDa}$ protein band observed in co-precipitated proteins from wild-type testis (Fig. 3.18 A) was also missing demonstrating that this band indeed represents ODF1 and that ODF1 interacts with SPAG4 in testicular cells. Moreover, as positive control for anti-ODF1 antibody specificity bacterially expressed ODF1 fused to MBP (mODF1-MBP) was used that was detected by the antibody. To identify the protein region of SPAG4 responsible for 
interaction with ODF1 co-IP of transfected cells were performed. To this end, ODF1 fused to ECFP was either co-transfected with a plasmid encoding the C-terminal end of SPAG4 fused to HA (HA-SPAG4-C) or with a plasmid encoding the N-terminal end of SPAG4 fused to HA (HA-SPAG4-N). In both constructs the HA-tag is fused to the N-terminal end. Proteins were precipitated using anti-GFP antibodies, and bound proteins separated on denaturing polyacrylamide gels. Co-precipitation of both the C-terminal region of SPAG4 with ODF1 (Fig. $3.18 \mathrm{C}$ ) as well as the N-terminal region of SPAG4 with ODF1 (Fig. 3.18 D) was confirmed by immunodetection of the HA-tag. SPAG4-C ( 35 kDa) and SPAG4-N ( 25 kDa) were clearly identified in the bead bound fractions (Fig. 3.18 C and D, lanes b) but not in the control bead fractions in which the anti-GFP antibody was omitted (Fig. $3.18 \mathrm{C}$ and D, lanes bc). Additionally, SPAG4-C and SPAG4-N were also detected in the soluble protein fraction (Fig. 3.18, lane s), in the insoluble protein fraction (Fig. 3.18, lane p), and in the depletion control fractions obtained after protein $G$ agarose mediated protein precipitation (Fig. 3.18, lanes dc). Incubation of Western blots with anti-GFP antibodies confirmed the presence of ODF1 in all fractions. Additionally, very faint signals were also obtained in the control experiments (Fig. 3.18, lanes bc). These results indicate that ODF1 is able to interact with both the C-terminal as well as the N-terminal end of SPAG4. 


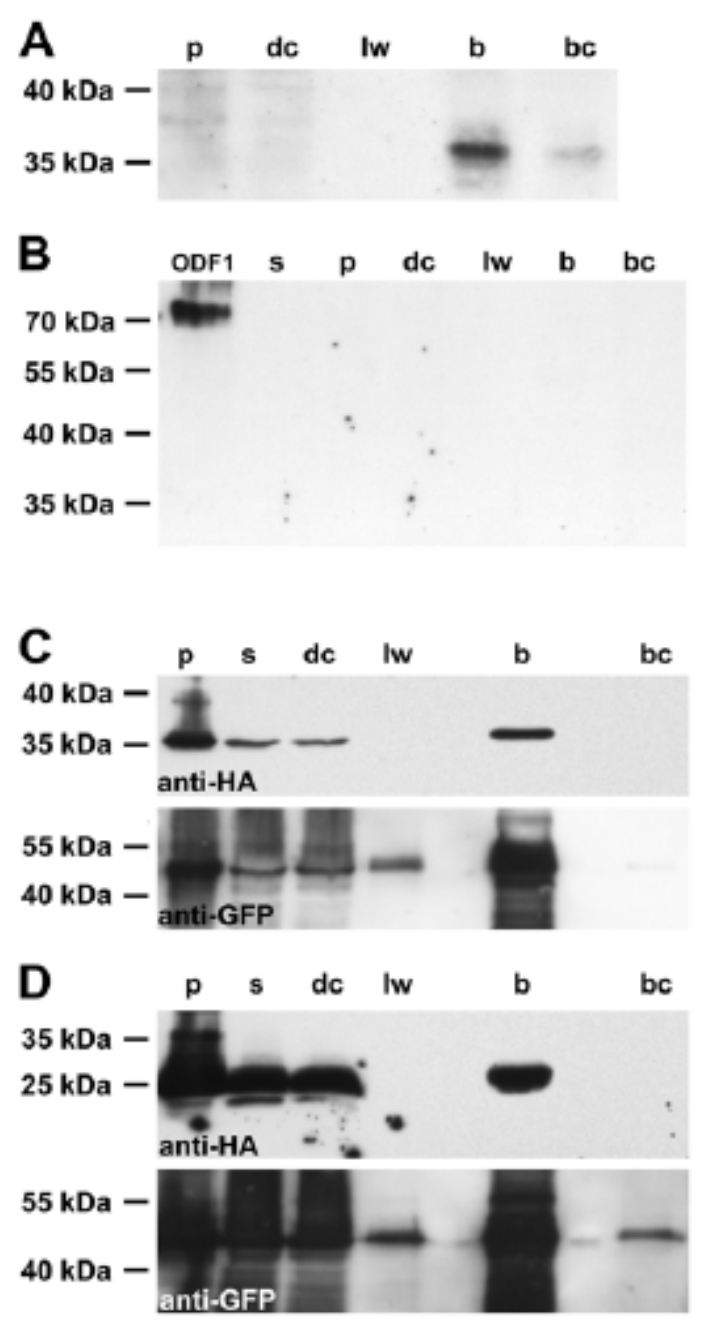

Fig. 3.18: Interaction of ODF1 and SPAG4. (A) and (B), interaction of endogenous proteins. SPAG4 was immunoprecipitated from wild-type testis (A) or Odf1 $1^{-/}$testis (B) and bound ODF1 detected on Western blots. In wild-type testis ODF1 co-precipitated with SPAG4 and was therefore detected in the bead bound fraction (A, lane b) whereas in ODF1-deficient testis no signal of similar molecular mass was found (B). The antibody however reacted with bacterially expressed ODF1 fused to MBP (B, lane ODF1). (C) and (D), interaction of ODF1 with both the C-terminal as well as the N-terminal part of SPAG4. Plasmids encoding ODF1 fused to ECFP (pODF1-ECFP) and either the C-terminal part of SPAG4 fused to HA (pHA-Spag4-C) (C) or the N-terminal part of SPAG4 fused to HA (pHA-Spag4-N) (D) were transfected into HEK293 cells and co-immunoprecipitation performed using anti-GFP antibodies. Co-precipitation with ODF1-ECFP was found for both SPAG4-C (C, lane b) and SPAG4-N (D, lane b). In the control bead bound fractions (lanes bc) no SPAG4 proteins were found. s, supernatant of cell lysate; p, 
pellet, insoluble cellular fraction; dc, depletion control, proteins of the supernatants after separation of protein $\mathrm{G}$ agarose beads; lw, last wash; $b$, proteins bound to beads after incubation with fishing antibody; bc, control, proteins bound to beads without the presence of the fishing antibody.

\subsubsection{The recruitment of SPAG4 to the posterior pole of elongating spermatids is independent of ODF1}

To investigate whether absence of ODF1 affects the recruitment of SPAG4 to the nuclear membrane in round spermatids and more specifically the redistribution to the posterior pole in elongating spermatids the SPAG4 distribution in $O d f 1^{-/}$male germ cells was analysed. Suspensions were prepared from Odf1 knock out testis and cells incubated with anti-SPAG4 antibodies and anti- $\alpha$-tubulin antibodies (Fig. 3.19). SPAG4 showed a similar distribution as in germ cells from wild type testis (Fig. 3.17) with scattered nuclear membrane localization in round spermatids (Fig. 3.19 A) and progressive redistribution towards the posterior pole in elongating spermatids (Fig. $3.19 \mathrm{~B}$ to E). Additionally, SPAG4 is located in close vicinity to the forming manschette as already found in wild type germ cells (Fig. 3.17). It is also obviously to be seen that a substantial amount of SPAG4 is present in the cytoplasma of elongating spermatids (Fig. 3.17 E; Fig. $3.19 \mathrm{C}$ and D). With these results it seems that the recruitment of SPAG4 to the posterior pole of elongating spermatids is independent of ODF1. 


\section{alpha tubulin SPAG4 DAPI}
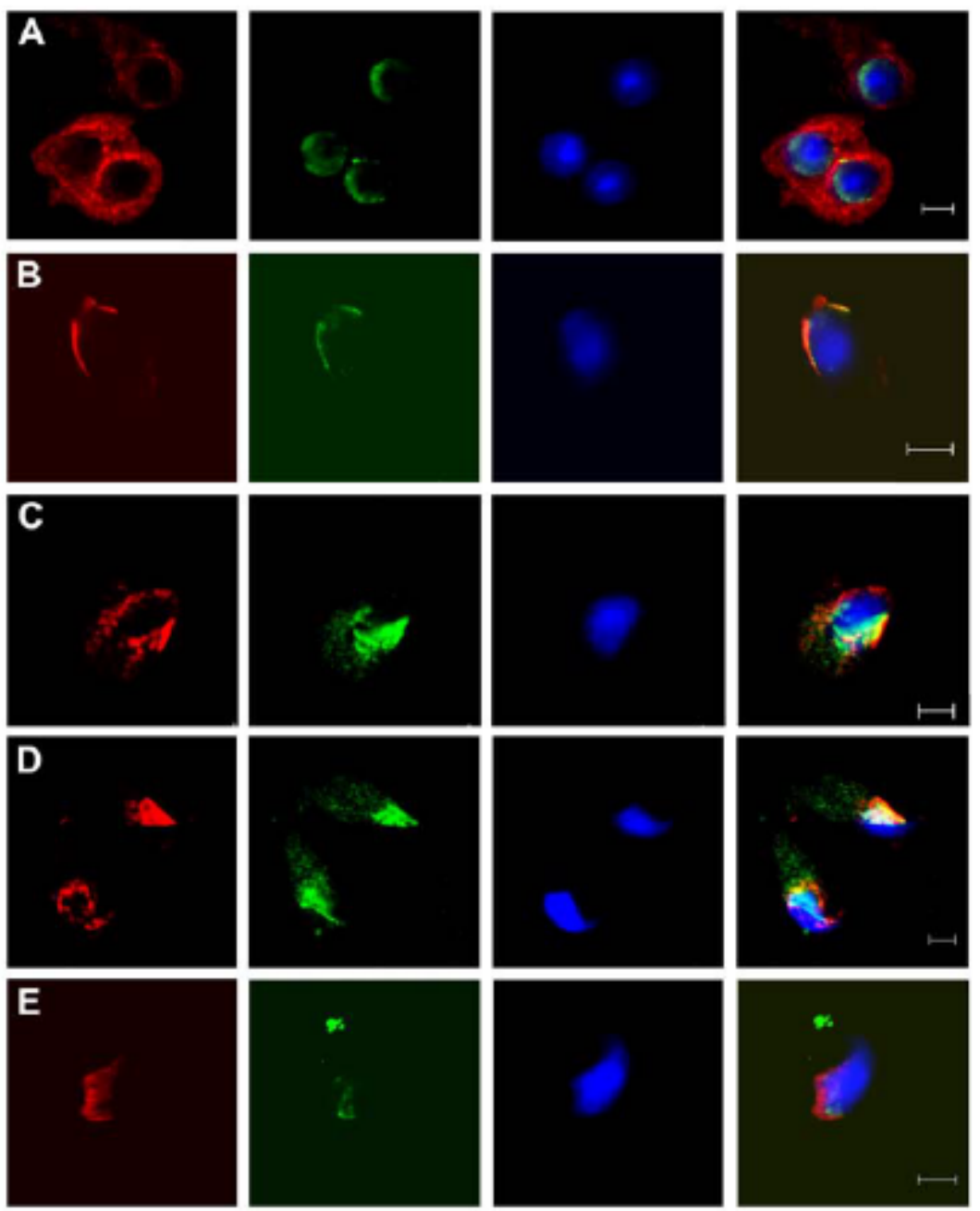

Fig. 3.19: Redistribution of SPAG4 towards the posterior pole of elongating spermatids is not mediated by ODF1. Testicular suspension preparations from Odf1 knock out mice were incubated with anti-SPAG4 antibodies (in green) and anti- $\alpha$ tubulin antibodies (in red). SPAG4 is first scattered over the round spermatid nucleus (A) and redistributed towards the posterior pole where the manschette is forming (B-E). An approximate colocalization with the manschette is found in elongating spermatids (C, D). In yet later spermatid stages the manschette disintegrated and SPAG4 became concentrated at the most distal pole (E). DAPI counterstain in blue. Scale bars are of $5 \mu \mathrm{m}$ each. 


\subsubsection{ODF1 is recruited to the nuclear membrane by SPAG4}

It was shown that recruitment of SPAG4 to the nuclear membrane and its concentration towards the posterior pole in elongating spermatids is not affected by absence of ODF1. Then the recruitment of ODF1 via SPAG4 to the nuclear membrane was also investigated. NIH3T3 cells were transfected with SPAG4 and ODF1 expression constructs and protein localization was analysed by immunocytology (Fig. 3.20). Whereas ODF1 (fused to ECFP) located to the cytoplasm but not to the nuclear membrane in cells without SPAG4 expression (Fig. 3.20 A), co-expression of full length SPAG4 (HA-SPAG4-Myc) recruited ODF1 to the nuclear membrane. Co-expression of the C-terminal part of SPAG4 (HA-SPAG4-C) demonstrated co-localization with ODF1 in the cytoplasm but no nuclear membrane staining (Fig. 3.20 C). In contrast, the N-terminal part of SPAG4 (HA-SPAG4-N) is localized to the nuclear membrane causing in turn recruitment of ODF1 to the nuclear membrane as well (Fig. 3.20 D). These results thus demonstrate that ODF1 and SPAG4 interact, that the N-terminal part of SPAG4, which comprises the predicted transmembrane domains, is indeed targeted to the nuclear membrane, and that SPAG4 recruits ODF1 to the nuclear membrane.

These investigations further revealed that ectopic expression of ODF1 resulted in a distinct concentration of ODF1 in the vicinity of the nucleus that reminds to the centrosome (Fig. 3.20 A, C, D, arrow). Therefore the cells transfected with Odfl was costained with anti- $\gamma$ tubulin antibody which detects the centrosomal protein $\gamma$ tubulin (Fig. 3.20 E). The arrow in panel E indicates that ODF1 is concentrated in a tiny dot near the nucleus that is $\gamma$ tubulin positive demonstrating that ODF1 is recruited to the centrosome. 


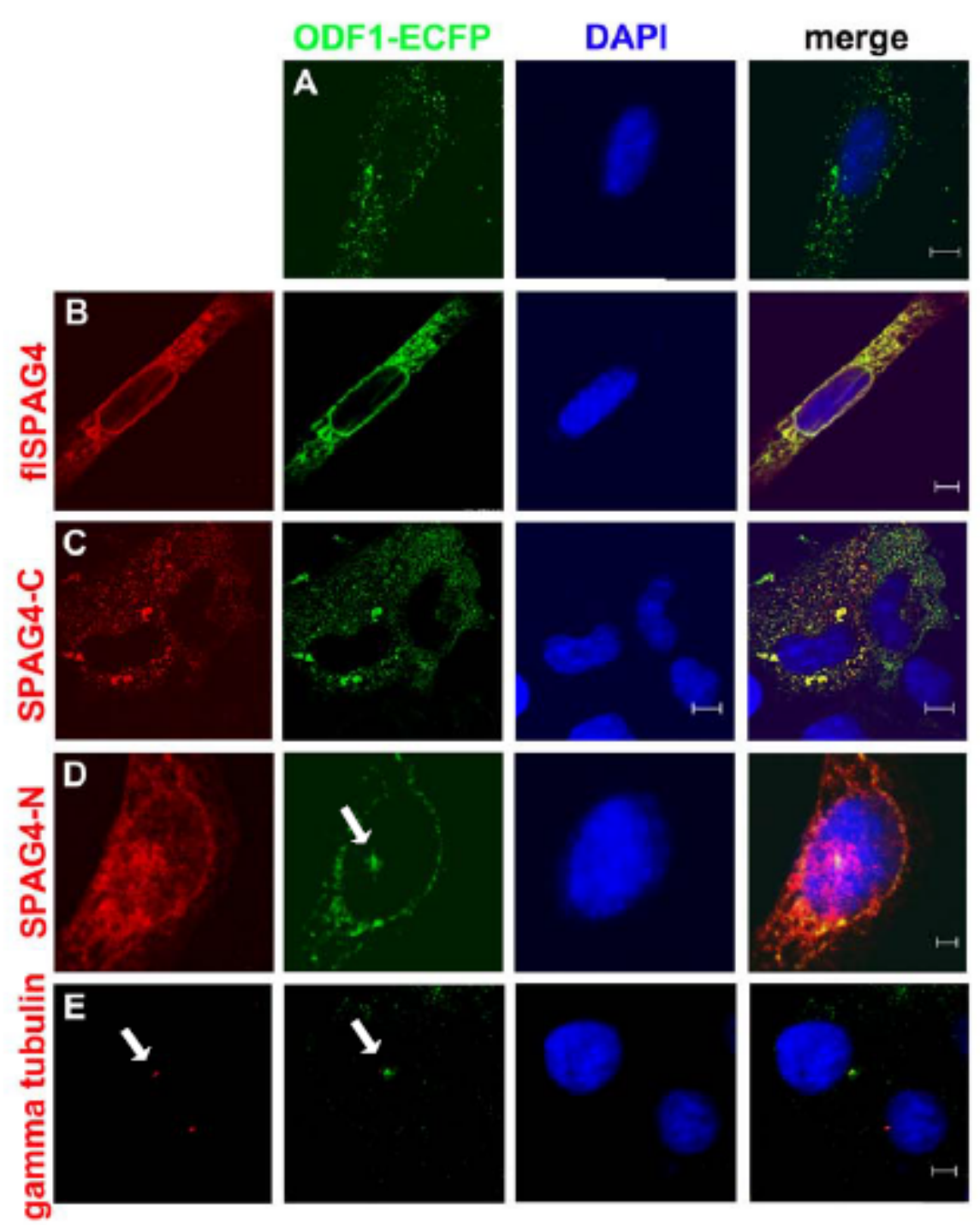

Fig. 3.20: Ectopically expressed ODF1 located at the centrosome and is recruited to the nuclear membrane by SPAG4 (full length, flSPAG4) and its N-terminal region. Cells were transfected with expression plasmids encoding ODF1 fused to ECFP ( $p$ Odf1-ECFP) and SPAG4. SPAG4 proteins were detected by anti-HA (in red) and ODF1 by anti-ODF1 or anti-GFP antibodies (in green). ODF1 is recruited to the nuclear membrane by the full length SPAG4 (B) as well as by the N-terminal region of SPAG4 (D). The C-terminal region of SPAG4 is located in the cytoplasm and co-localized with ODF1 (C). Without SPAG4 ODF1 is cytoplasmic (A). ODF1 is concentrated in a tiny dot near the nucleus (arrows in D and E) that co-localizes with $\gamma$ tubulin (E, in red). DNA counterstain with DAPI. Scale bars are of $2 \mu \mathrm{m}$ (D), $5 \mu \mathrm{m}$ (A, B, E) or of $10 \mu \mathrm{m}$ (C). 


\subsection{ODF2 protein}

\subsubsection{The influence of centrosomal protein ODF2 on canonical Wnt pathway key protein $\beta$-catenin}

\subsubsection{Ectopic expression of ODF2 promotes the degradation of over-expressed $\beta$-catenin}

Previous results from pull-down and co-immunoprecipitation showed that ODF2 interacts with $\beta$-catenin (colleagues of our group). I subsequently investigated the influence of ODF2 on $\beta$-catenin. Mammalian cell lines NIH3T3 and HEK293 were used for transfection of different plasmid DNA constructs, then reporter gene assay was performed. The reporter gene Topflash $(O T)$ is firefly-luciferase which has a promoter with Tcf4 binding sites. When the canonical Wnt signaling pathway is activated, $\beta$-catenin goes into the nucleus and binds to the Tcf4 binding site with Tcf4 together and the reporter gene will be subsequently activated. Then the luciferase activity may be measured by luminometer.

The over-expressed $\beta$-catenin can cause an obvious increasing of firefly-luciferase activity. After the co-transfection of $\beta$-catenin and $O d f 2(13.8 N C-G F P)$ the relative activity of luciferase reporter is strongly reduced from $100 \%$ down to $51 \%$ and to 39\% in NIH3T3 and HEK293 cells, respectively, compared to single transfection of $\beta$-catenin (Fig. $3.21 \mathrm{~A}$ and B). This result suggests that over-expression of ODF2 affects $\beta$-catenin stability.

$\beta$-catenin is a key protein of canonical Wnt pathway. When canonical Wnt pathway is not activated, cytoplasmic $\beta$-catenin will be targeted for degradation by a destruction complex containing APC, CK1, Axin and GSK-3 $\beta$. Therefore, I examined whether the influence of ODF2 on the canonical Wnt pathway is mediated by the $\beta$-catenin destruction complex. Lithium potently inhibits GSK-3 $\beta$ activity, but is not a general inhibitor of other protein kinases (Klein and Melton, 1996). The cells were treated with $20 \mathrm{mM}$ lithium acetate for 16 hours. Once GSK-3 $\beta$ is inhibited by lithium, $\beta$-catenin will not be degraded and acts as a transcriptional activator. Fig. $3.21 \mathrm{~A}$ and 
B show that in lithium-treated cells (NIH3T3 and HEK293) over-expressed ODF2 obviously reduces the activity of the luciferase reporter from $100 \%$ down to $72 \%$ and to $40 \%$ in NIH3T3 and HEK293 cells, respectively, compared to single transfection of $\beta$-catenin. This reduction demonstrates that the influence of ODF2 on $\beta$-catenin is not exclusively mediated by GSK-3 $\beta$.

To support the results of reporter gene assays, I investigated subsequently $\beta$-catenin expression quantified by Image $\mathbf{J}$ software using $\alpha$-tubulin expression as loading control. Western blot analyses showed that over-expressed ODF2 did not affect the endogenous $\beta$-catenin neither in NIH3T3 nor in HEK293 cells. Therefore, $\beta$-catenin was over-expressed in HEK293 cells. The results indicated that co-transfection of Odf 2 and $\beta$-catenin together triggered a decrease of $\beta$-catenin, which was $76 \%$ of $\beta$-catenin found in cells transfected exclusively with $\beta$-catenin (Fig. 3.21 C, D). Moreover, the endogenous $\beta$-catenin was not affected by ectopically over-expressed ODF2. The relative amount of degraded $\beta$-catenin fragments inversely corresponded to the relative amount of intact $\beta$-catenin: less intact $\beta$-catenin could be detected and more degraded $\beta$-catenin exists in cells which were transfected with $\beta$-catenin and 13.8NC-GFP. This result demonstrates that over-expressed ODF2 could abet the degradation of over-expressed $\beta$-catenin.

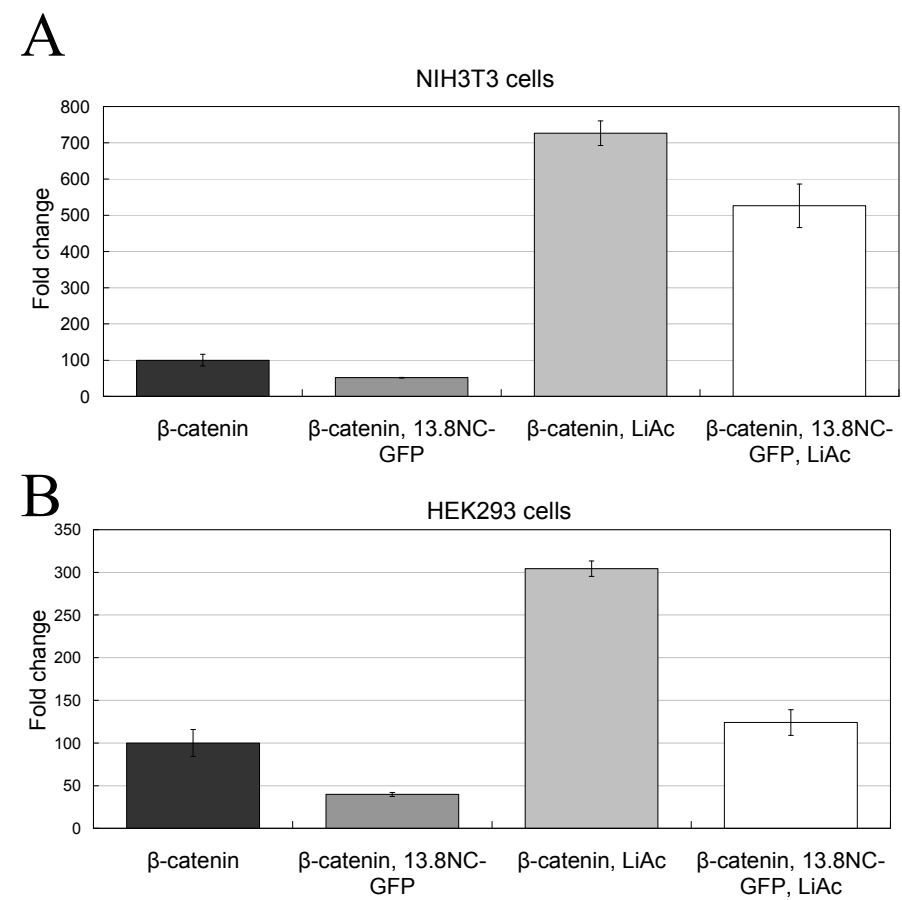




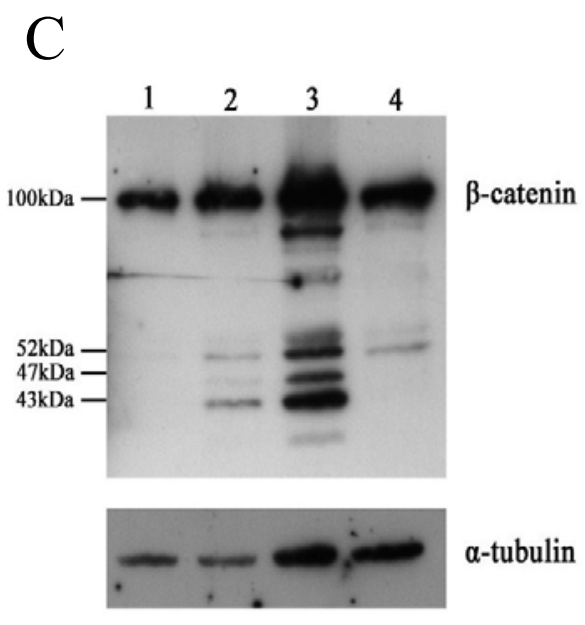

1. untransfected HEK293

2. HEK293 transfected with $\beta$-catenin

3. HEK293 transfected with $\beta$-catenin and $13.8 N C$-GFP

4. HEK293 transfected with $13.8 N C$-GFP

$\mathrm{D}$
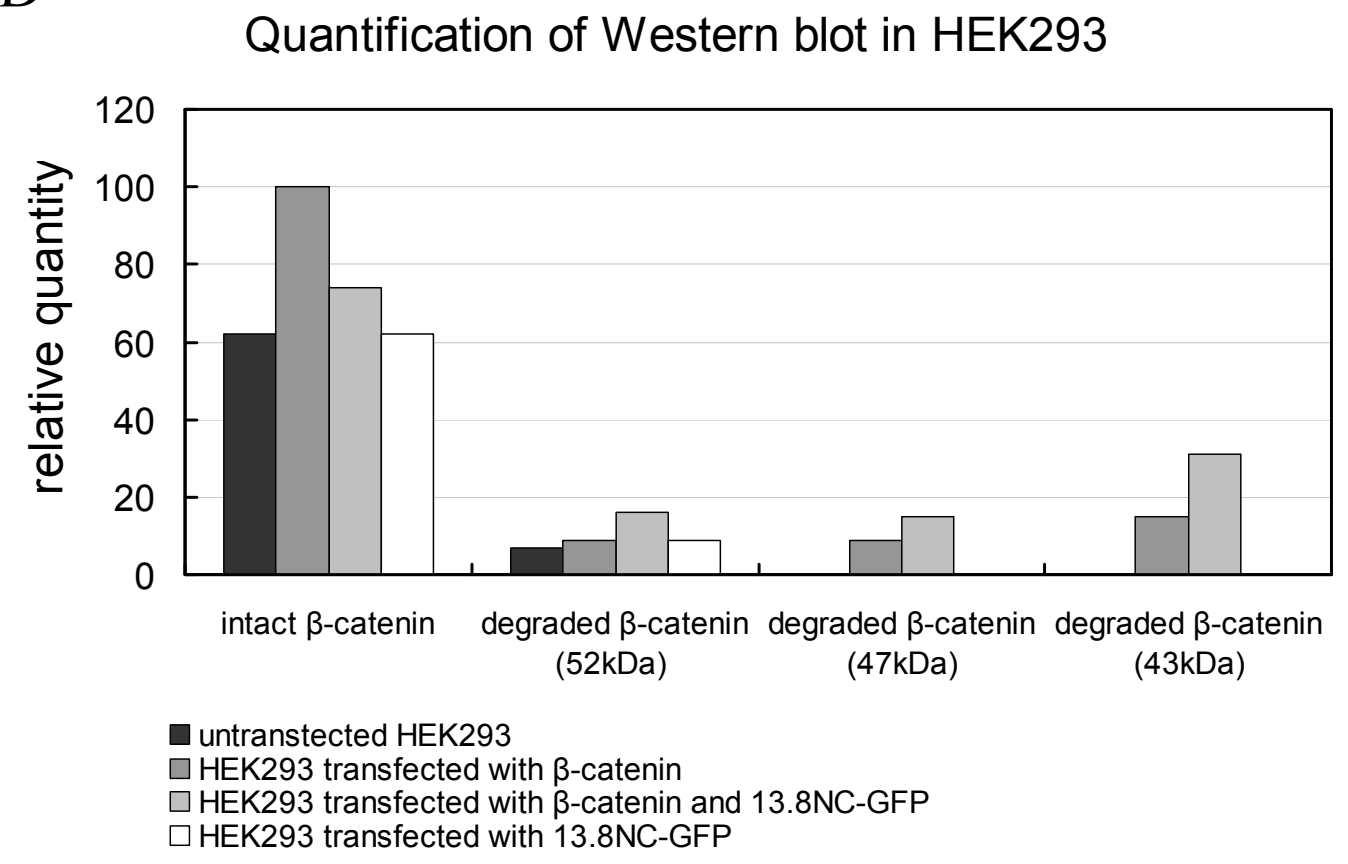

Fig. 3.21: The influence of over-expressed ODF2 on $\beta$-catenin activity. (A) and (B), reporter gene assay in NIH3T3 cells (A) and HEK293 cells (B). Over-expressed ODF2 decreases the activity of luciferase reporter in both cell lines and as well by lithium-treatment. (C), the expression of $\beta$-catenin in differently transfected HEK293 cells. The intact $\beta$-catenin is $\sim 100 \mathrm{kDa}$. The main degraded $\beta$-catenin fragments are $\sim 52 \mathrm{kDa}, \sim 47 \mathrm{kDa}$ and $\sim 43 \mathrm{kDa}$. $\alpha$-tubulin is the loading control. (D), the relative quantity results of $\beta$-catenin expression normalized to $\alpha$-tubulin according to Western blot of figure 3.21 C. Quantifications are divided into four groups according to the $\beta$-catenin fragment size. 


\subsubsection{A high ODF2 expression level abets the degradation of endogenous $\beta$-catenin}

Cycloheximide is an inhibitor of protein biosynthesis in eukaryotic organisms and exerts its effect by interfering with the translocation step in protein synthesis thus blocking translational elongation. HEK293 cells were transfected with $13.8 N C-G F P$ and incubated for 18 hours until a large amount of exogenous ODF2 fused to GFP was translated. Afterwards untransfected and transfected cells were treated with cycloheximide $(100 \mu \mathrm{g} / \mathrm{ml})$ for $0,4,8,12$ and 24 hours, respectively. After cycloheximide treatment, the proteins extracted from cells were examined by Western blotting (Fig. 3.22 A). Since cells were treated with cycloheximide, protein synthesis was inhibited.

Fig. 3.22 B shows that transfected cells had an increasing relative exogenous ODF2 expression level in all cycloheximide treatments from 0 hour to 24 hours. This increase might be caused by a faster degradation of $\alpha$-tubulin rather than over-expressed ODF2. Interestingly, in transfected cells, a higher ODF2 expression level corresponds to a lower $\beta$-catenin expression level (at 0,12 , and 24 hours) and vice versa (at 4 and 8 hours). Moreover, $\beta$-catenin was more degraded in transfected cells than in untransfected cells obviously from 8 to 24 hours. These results indicate that high ODF2 expression level promotes the degradation of endogenous $\beta$-catenin. 
untransfected cells

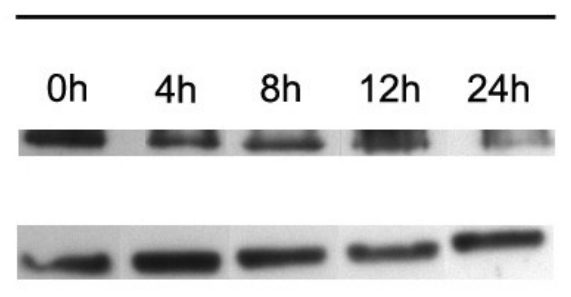

cells transfected with 13.8NC-GFP

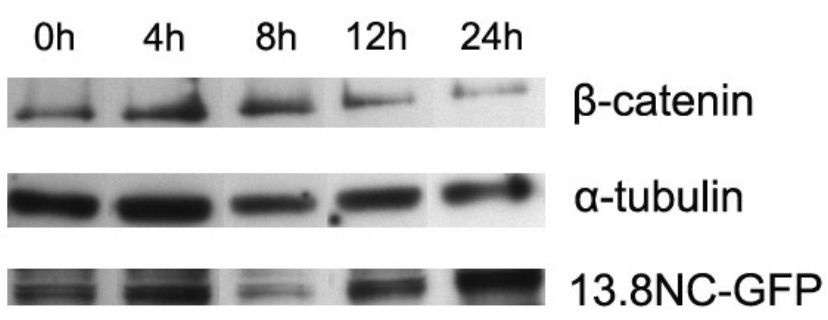

B

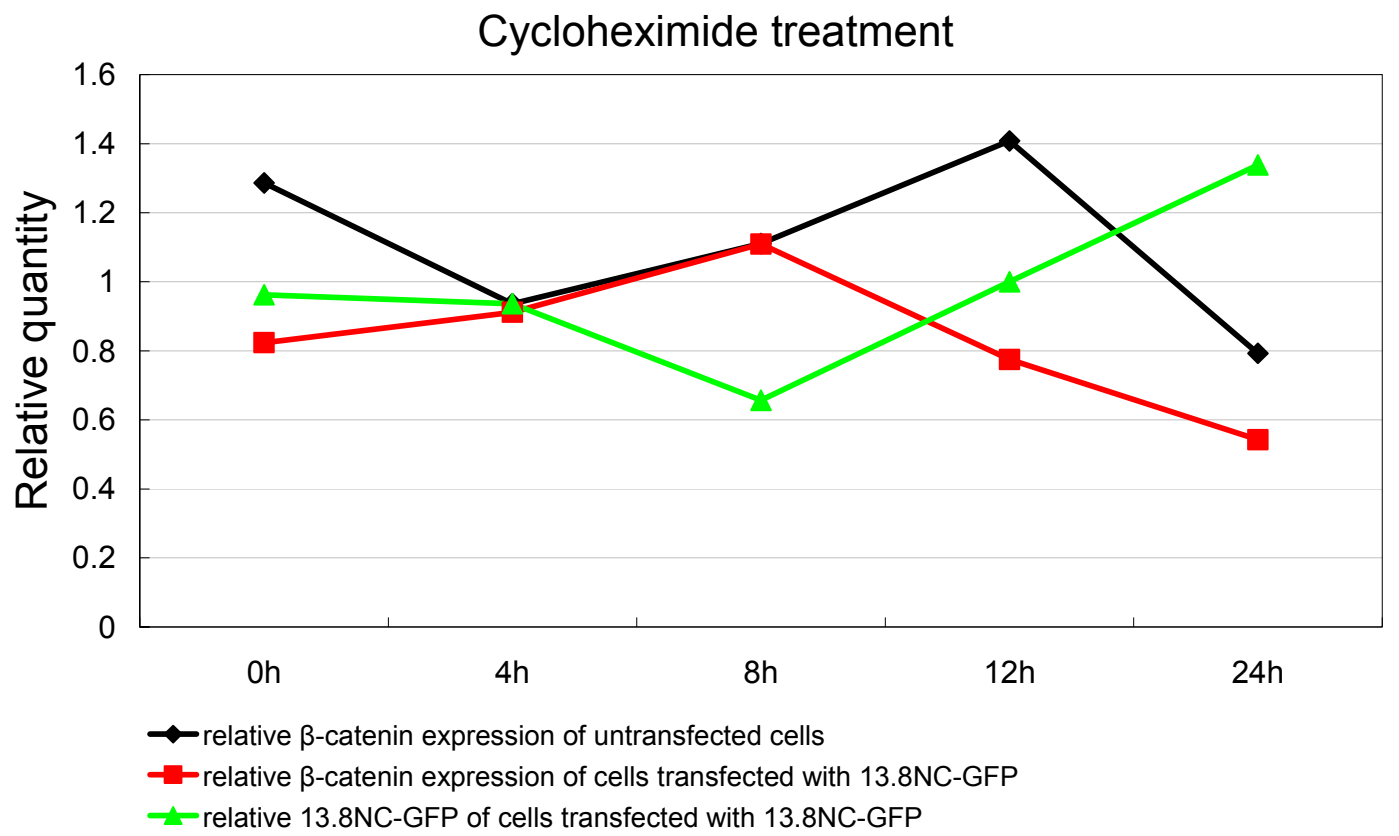

Fig. 3.22: Relative quantity of $\beta$-catenin in HEK293 cells treated with cycloheximide. (A), detection of intact endogenous $\beta$-catenin $(\sim 100 \mathrm{kDa})$ in untransfected cells and cells transfected with $13.8 N C$-GFP after cycloheximide treatment ( $0 \mathrm{~h}, 4 \mathrm{~h}, 8 \mathrm{~h}, 12 \mathrm{~h}$ and $24 \mathrm{~h}$, respectively). $\alpha$-tubulin as loading control. The ectopic ODF2 expression level of cells transfected with $13.8 N C$-GFP was inspected with anti-GFP antibody. (B), relative quantities of $\beta$-catenin and ODF2, respectively, normalized to $\alpha$-tubulin according to the Western blot of figure 3.22 in panel A. 


\subsubsection{Investigation of ODF2 protein domains responsible for canonical Wnt pathway inhibition}

ODF2 and Cenexin are alternative splice products distinguished by a novel exon $3 \mathrm{~b}$ encoding 42 amino acids present exclusively in Cenexin isoforms (cenexin insertion) (Hüber and Hoyer-Fender, 2007). The cenexin insertion is necessary for targeting ODF2/Cenexin to the centrosome and primary cilium, and also for the formation of ODF2/Cenexin fibers that are associated with acetylated microtubules (Hüber et al., 2008). Reporter gene assays were employed to investigate whether the cenexin insertion is also necessary for the down-regulation of canonical Wnt pathway. A series of deletion constructs were generated and fused to GFP by former lab members in our group (Fig. 3.23 A, Donkor et al., 2004; Hüber et al., 2008). Each construct was co-transfected with $\beta$-catenin into HEK293 cells and reporter gene assays were performed (Fig. $3.23 \mathrm{~B}$ ).

Among these constructs, the first 86 amino acids of N-terminus of ODF2 protein and the human testis-isoform of ODF2 that missed cenexin insertion (42 aa) have a minimal down-regulative effect on the activity of the reporter gene compared to the control (phRL, OT, $\beta$-catenin) and any other of the co-transfections (Fig. $3.23 \mathrm{~B})$. The coiled-coil part (shown in black in Fig. 3.23 A) showed a strong repression. The cenexin insertion by itself repressed canonical Wnt pathway whereas the first 44 amino acids of $\mathrm{N}$-terminal end seems to reduce the overall repressive effect. 
A

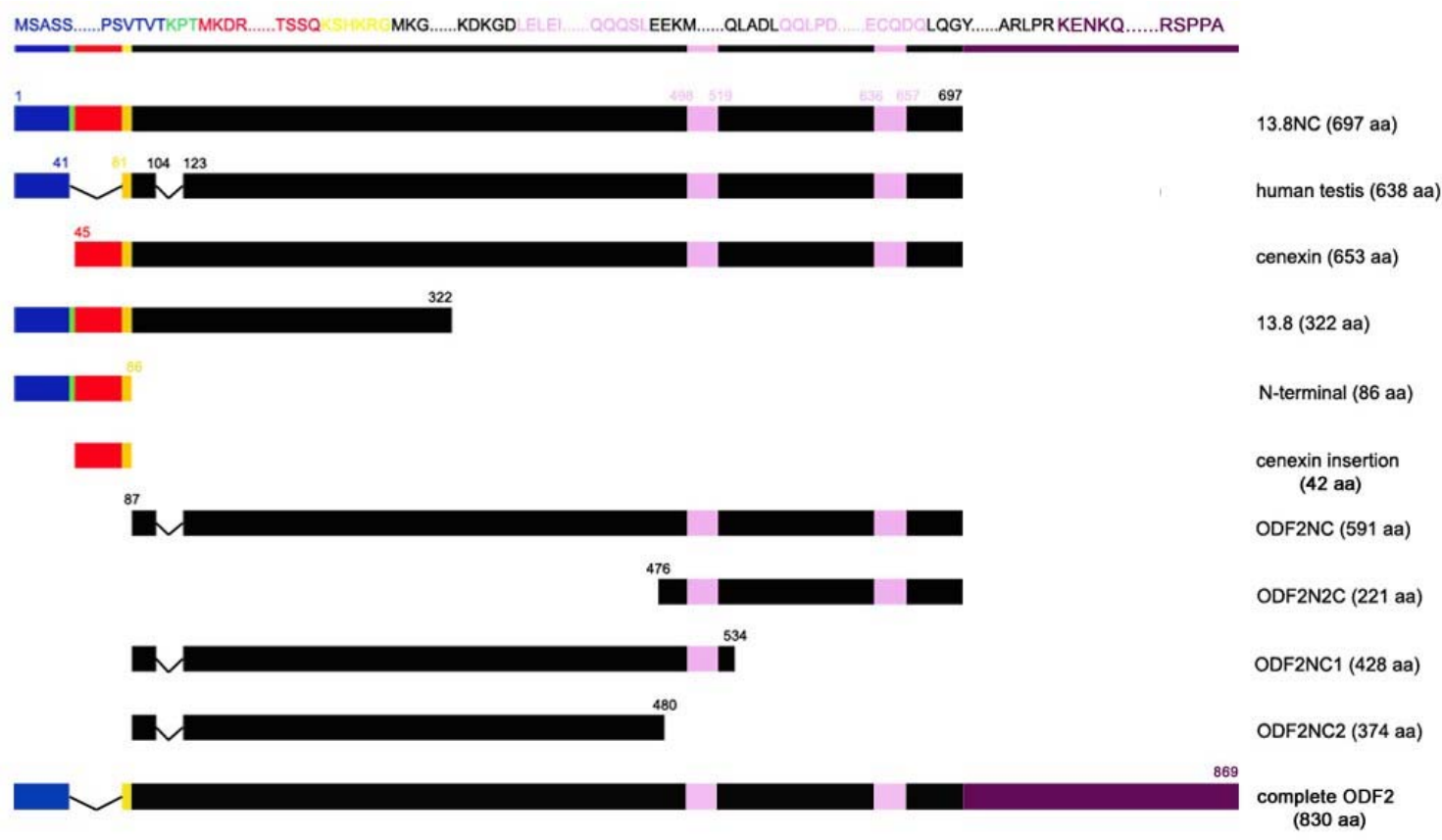

B

\section{Odf2 constructs}
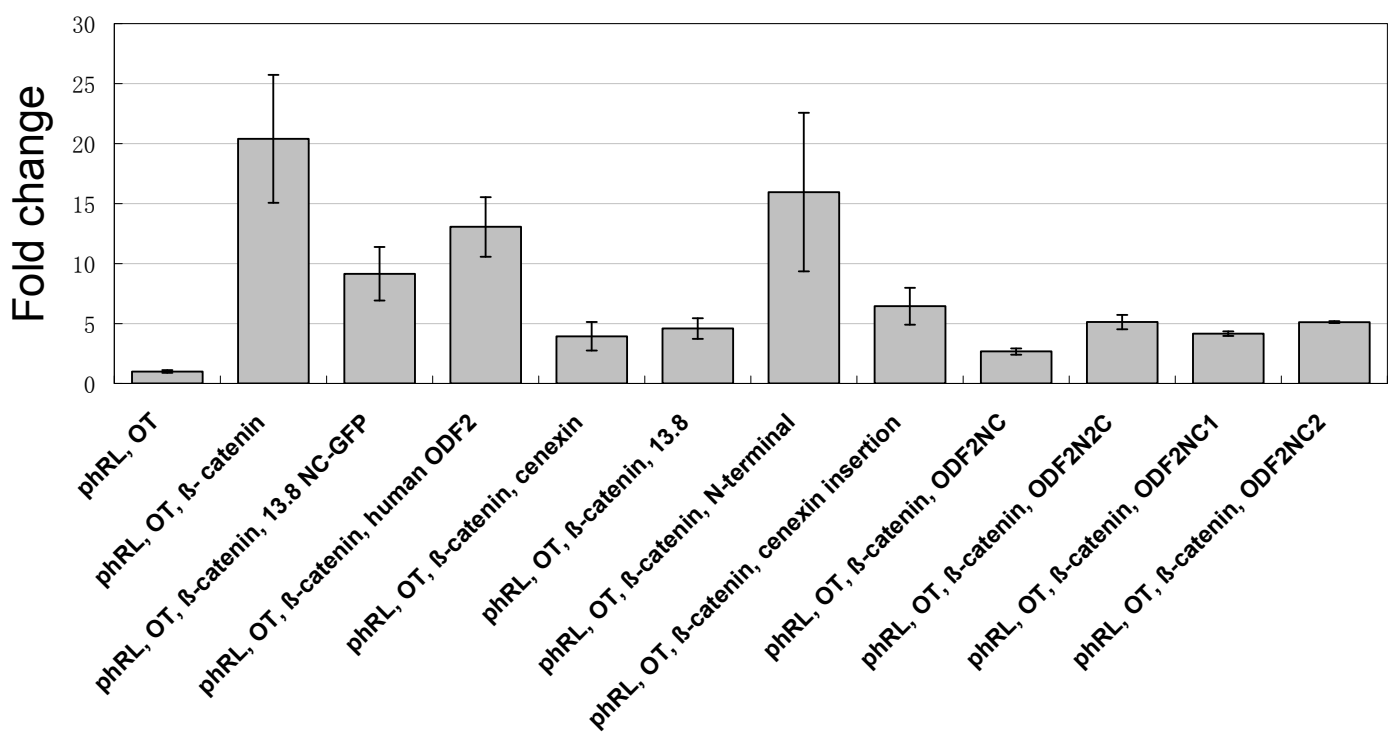

Fig. 3.23: The influence of different ODF2 protein domains translated from a series of deletion constructs on canonical Wnt pathway. (A), schematic drawing of a series of deletions translated from ODF2 constructs. 13.8NC: ODF2 protein starting with MSASS and comprising cenexin insertion. Human testis: the ODF2 isoform localizing specially in sperm. cenexin: the 
$\mathrm{N}$-terminal region of ODF2 isoform expressed in somatic cells. 13.8, N-terminal, cenexin insertion, ODF2NC, ODF2N2C, ODF2NC1 and ODF2NC2: they were encoded by homonymous subclones of Odf2 (Donkor et al., 2004; Hüber et al., 2008). Sequences 498-519 and 636-657 are two leucine-zipper motifs. (B), canonical Wnt reporter gene assay using Odf2 constructs shown in panel A. The different $O d f 2$ constructs were co-transfected with $\beta$-catenin into HEK293 cells and the relative reporter gene assay activity quantified 24 hours later (cooperation with bachelor student Annie Angelique Nono Megaptche).

\subsubsection{Centrosomal proteins and cytoskeletal proteins down-regulate the canonical Wnt reporter}

Since I have shown that ODF2 down-regulates the activity of $\beta$-catenin, the question arises: whether other centrosomal proteins also affect the activity of $\beta$-catenin. I have chosen the centriolar proteins Ninein, Centrin and $\varepsilon$-tubulin and pericentriolar material proteins $\gamma$-tubulin (Paul Chang and Tim Stearns, 2000) to investigate their influences on reporter gene activity in HEK293 cells. Ninein localizes at the subdistal appendages of mother centrioles like ODF2 (Nakawaga et al., 2001; Morgensen et al., 2000). Centrin localizes in the lumen of centrioles (Paoletti et al., 1996). For each experiment the cells were transfected with $\beta$-catenin alone, and the relative luciferase activity set as $100 \%$. Relative luciferase activity of co-transfection experiments were related to the control value. Fig. 3.24 A shows that over-expressed Ninein, Centrin and $\varepsilon$-tubulin down-regulate the luciferase activity to $35 \%, 40 \%$ and $39 \%$, respectively. $\gamma$-tubulin decreases the luciferase activity down to $7 \%$. This result indicates that there might be a tight relationship between the canonical Wnt pathway and centrosomal proteins.

Fig. 3.24 A also shows that over-expressed $\alpha$-tubulin, which is a main microtubular protein, can strongly inhibit the activity of luciferase reporter from $100 \%$ down to $8 \%$.

Nocodazole is a chemical that interferes with the polymerization of microtubules in 
cells. When microtubule proteins negatively affect the activity of the reporter, nocodazole probably could rescue it due to disturbing the polymerization of microtubules. The cells were therefore treated by $100 \mathrm{ng} / \mathrm{ml}$ nocodazole for 16 hours. Nocodazole can indeed increase the reporter luciferase activity at $\sim 20 \%$ (Fig. 3.24 B, colorless group). Likewise, the decreased luciferase activities caused by over-expression of either centrin, $\alpha$-tubulin or $\gamma$-tubulin were partially rescued by nocodazole as well (Fig. 3.24 B, yellow, green and red groups, respectively). This result argues that the microtubule system negatively affects canonical Wnt signaling and that the disturbance of microtubule polymerization partially negotiates its inhibitory effect.

In opposite to nocodazole taxol stabilizes microtubules. When microtubules suppress the activity, the stabilization of microtubules should principally enhance this inhibition effect. The HEK293 cells were treated with 100nM taxol for 16 hours. Surprisingly the activity of reporter luciferase did not decrease but conversely had a $\sim 50 \%$ increase after the taxol treatment (Fig. 3.24 B, colorless group). 
A
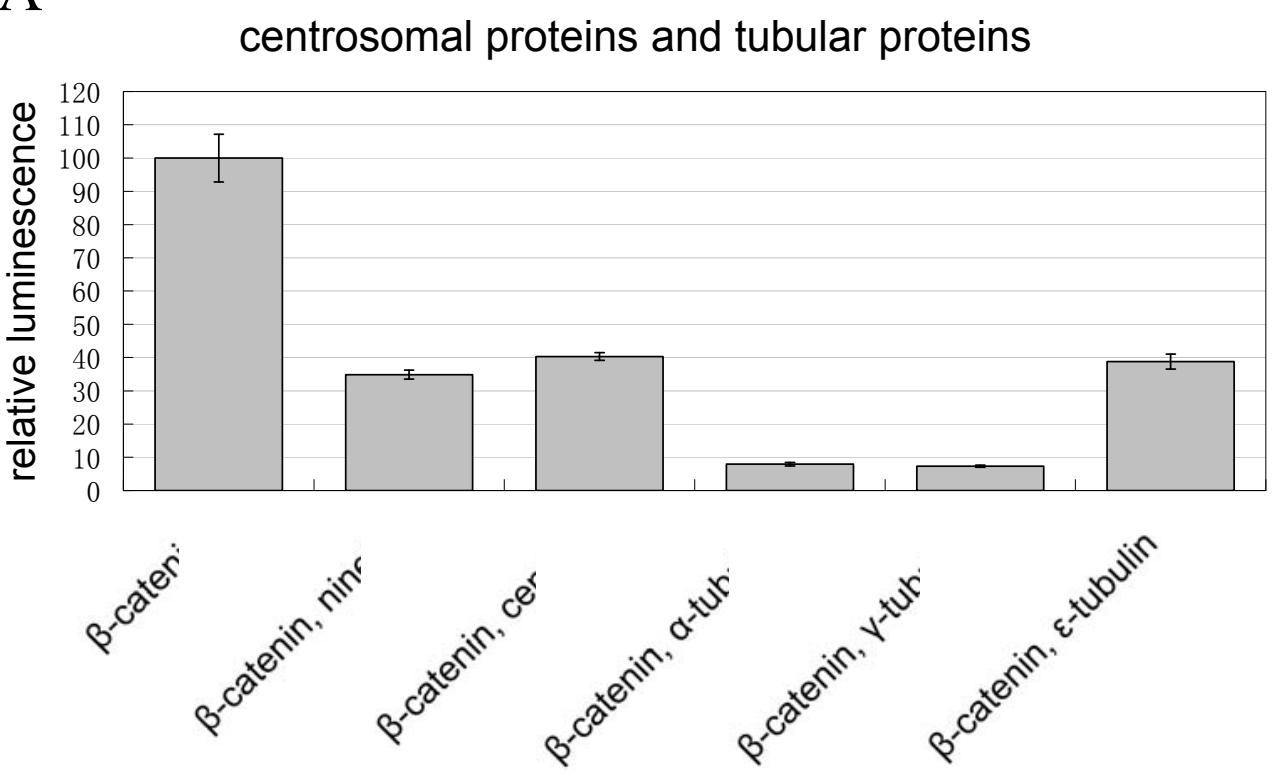

B
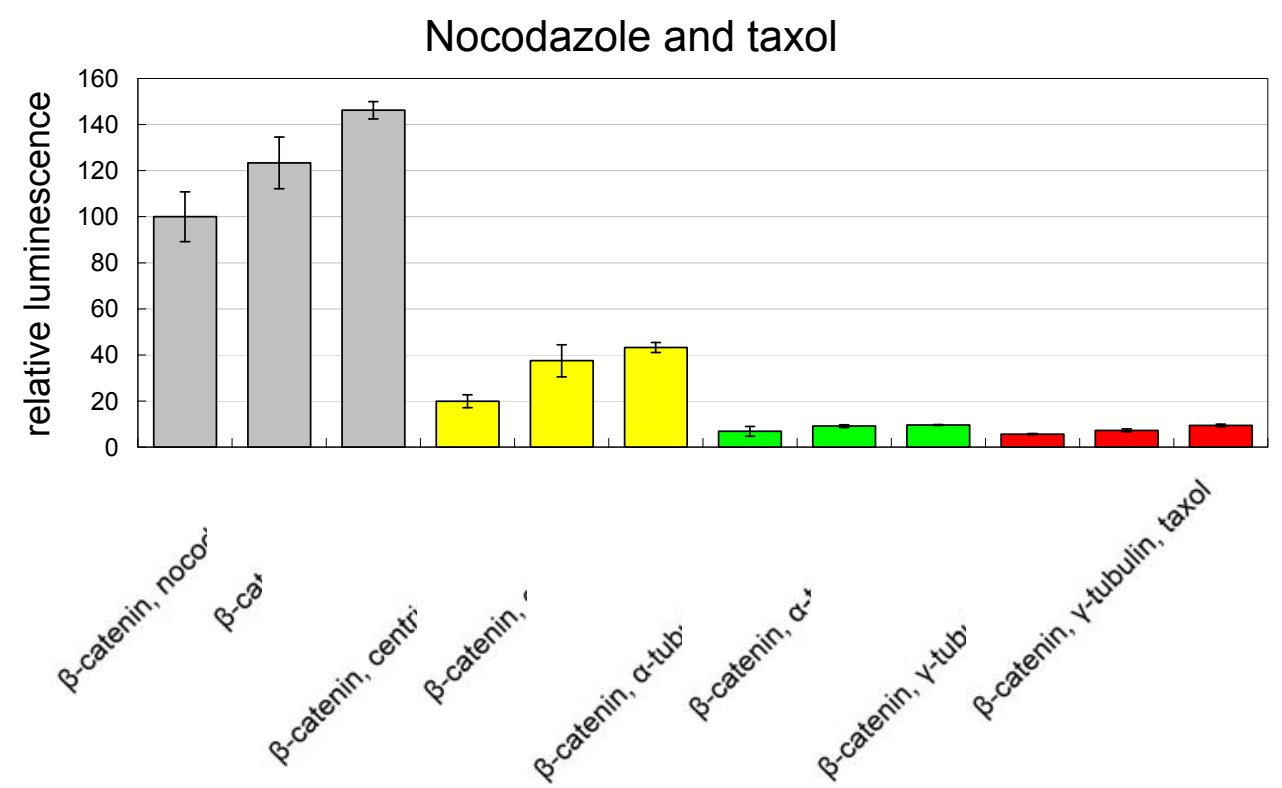

Fig. 3.24: Analysis of the influence of centrosomal and cytoskeletal proteins on canonical Wnt reporter. (A), the activity of over-expressed $\beta$-catenin can be down-regulated by centrosomal proteins and microtubule proteins. (B), nocodazole and taxol can rescue $\beta$-catenin activity to a certain extent that was otherwise inhibited by over-expression of either Centrin, $\alpha$-tubulin, or $\gamma$-tubulin. Nocodazole, 100ng/ml. Taxol, 100nM. 


\subsubsection{The influence of over-expressed ODF2 on target genes of the canonical Wnt pathway}

\subsubsection{The over-expression of ODF2 does not obviously affect the activation of canonical Wnt pathway target genes in vivo (Xenopus laevis)}

When $\beta$-catenin accumulates in the cytoplasm, it will enter into the nucleus and activates as transcriptional co-activator of the transcription factors TCF/LEF target genes of the canonical Wnt pathway. Therefore, I subsequently investigated the effect of over-expressed ODF2 on down-stream target genes of the canonical Wnt pathway. In Xenopus laevis, a complete second dorsal body axis can be induced by over-expression of Xwnt-8, which belongs to the Wnt family of secreted glycoproteins. Microinjection of Xwnt-8 mRNA can rescue axial structures in embryos ventralized by UV irradiation (Sokol et al., 1991; Smith and Harland, 1992). I co-injected the mRNA of Xwnt-8 and Odf2 into the embryos of Xenopus laevis to observe whether the over-expressed ODF2 can avoid or reduce the presence of the secondary axis at their tadpole stages.

About 4 days after injection, the embryos enter the tadpole stages and develop the secondary dorsal axis. Injection of 500pg Odf2 mRNA caused lethality before they entered into the tadpole stages. Therefore, the amount of $O d f 2$ mRNA used for injection was reduced to $200 \mathrm{pg}$ at the utmost. Fig. 3.25 shows that there is no significant difference between single injection of Xwnt-8 (8pg) and co-injection of Xwnt-8 (8pg) and Odf2 (50pg, 100pg and 200pg, respectively), regarding secondary axis formation. However, an increased Odf2 mRNA amount injected slightly but gradually reduced secondary dorsal axis formation $71 \%$ to $65 \%$ (Fig. 3.25). This result demonstrates that over-expressed ODF2 had a moderate effect on Xwnt-8 induced secondary axis formation in Xenopus embryos. 


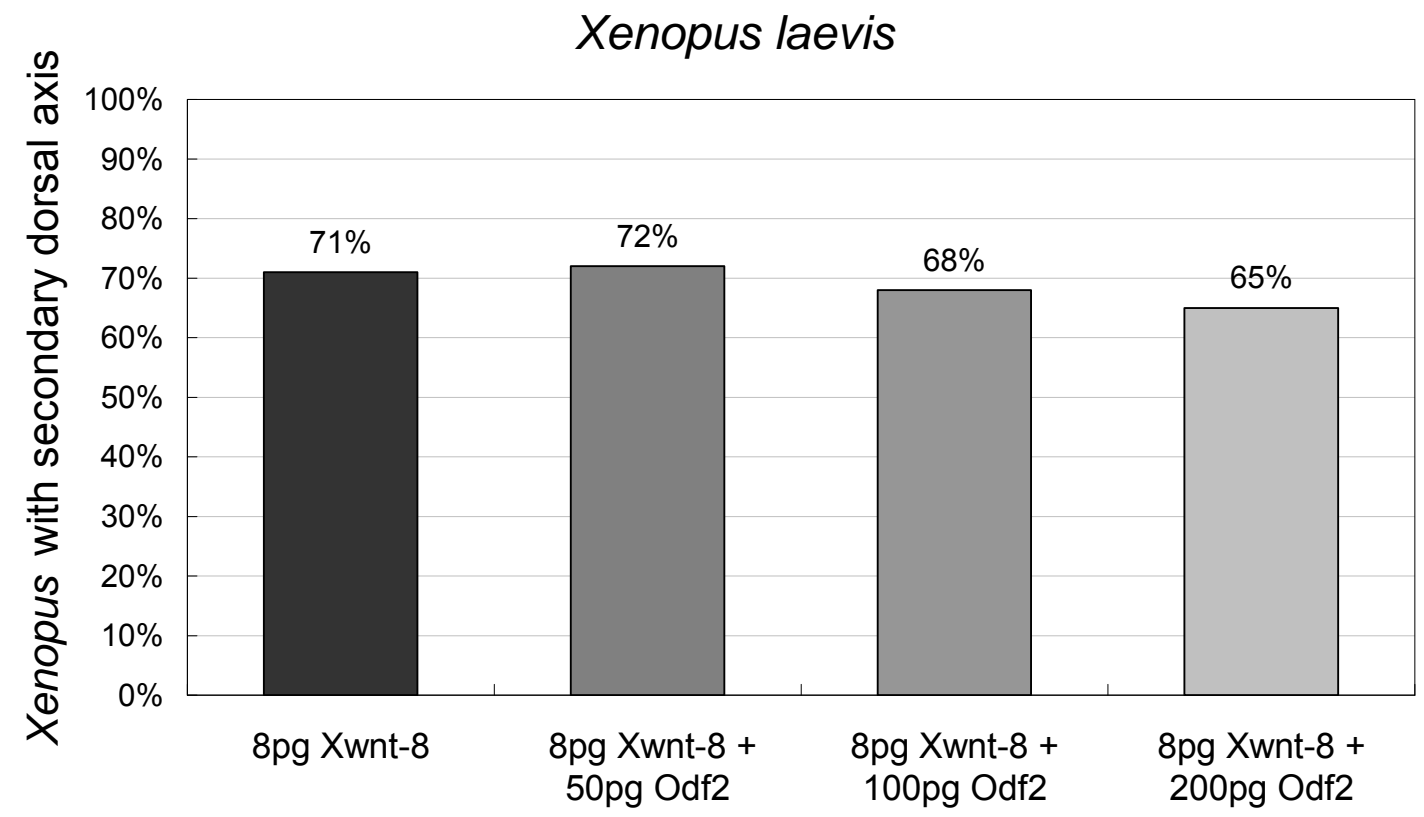

Fig. 3.25: The comparison of single injection of Xwnt-8 (8pg) mRNA and the co-injection of Xwnt-8 (8pg) and different amounts of Odf2 mRNA (50pg, 100pg and 200pg, respectively) on secondary axis formation (cooperation with Dr. B. Rust, Henningfeld's group).

\subsubsection{Over-expression of ODF2 inhibits $c$-Myc transcription}

Here, $c-M y c$ was chosen as Wnt target gene and $H P R T$ as house keeping gene for expression level analysis using real time PCR. HEK293 cells were transiently transfected with one of five different plasmid constructs, respectively: 13.8NC-GFP, Odf2 cloned into $p E G F P-N 1$ vector; 13.8NCAGFP, Odf2 cloned into $p E G F P-N 1$ vector without GFP; $p E G F P-1$, which does not have a functional promoter; pEGFP-N1, the empty $p E G F P-N 1$ vector; $s h O D F 2$, short hairpin Odf2 cloned into pIRES-EGFP vector. Besides these transient transfections, the construct 13.8NC-GFP was stably tansfected into HEK293 cells using neomycin selection. Untransfected HEK293 cells were used as blind control in real time PCR.

Two independent relative real time PCR experiments were arranged, each performed in triplicate. Fig. 3.26 shows that cells transfected with $p E G F P-1$, which does not have a functional promoter, have a reduced expression of $c-M y c$. This suggests that 
the expression of $c-M y c$ was generally affected by the transfection reagent. Ectopically expressed ODF2 (13.8NC-GFP and 13.8NCAGFP, respectively) down-regulate the transcription of $c-M y c$. However GFP (empty $p E G F P-N 1$ vector) also has a negative effect on the transcription of $c-M y c$. However, transcription of $c-M y c$ was strongly suppressed in cells that were stably transfected with $13.8 N C-G F P$. Unexpectedly, the lower expression of ODF2 via shODF2 interference did not promote the activation of $c-M y c$, but reduced its transcription level when compared to untransfected cells. However, when compared to cells transfected with 13.8NC-GFP, transcription of $c-M y c$ was partially rescued by $s h O D F 2$ interference. Taken all together, this result indicates that the down-regulation of $\beta$-catenin by over-expression of ODF2 leads to a reduced transcription level of the canonical Wnt target gene $c-M y c$.

It was reported that at least $15 \%$ of breast cancers present distinct amplification of $c-M y c$ and $c-M y c$ is also amplified very frequently in many other tumors (Deming, et. al. 2000). This suggests that transcription level of $c-M y c$ might be higher in those cancers than in normal tissues. Therefore, the real time PCR was performed in two human breast cancer cell lines, MCF7 and MDA-MB-231, to check their transcription level of $c-M y c$ and whether over-expression of ODF2 has also an effect on $c-M y c$ in cancer cells. In MCF7 cells, untransfected and stably transfected (13.8NC-GFP), $c-M y c$ template copies were nearly same and extremely exiguous ( 20 copies / 20 $\mu \mathrm{g}$ cDNA). In MDA-MB-231 cells, untransfected and stably transfected (13.8NC-GFP), there was no $c-M y c$ cDNA template to be detected at all (data not shown). Therefore, the influence of over-expressed ODF2 on $c-M y c$ could not be confirmed in these two cell lines further. 
c-Myc gene expression in HEK293 cells

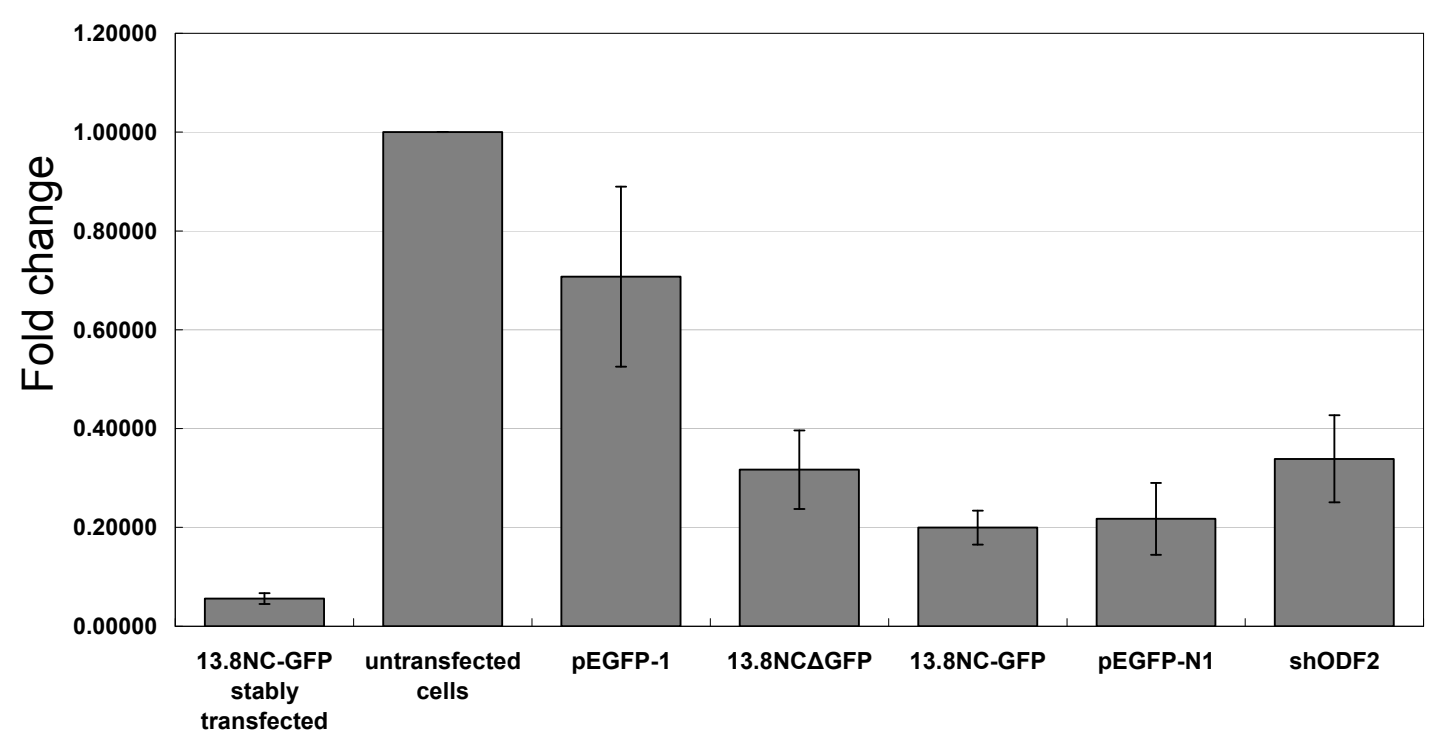

Fig. 3.26: Transcription of $c-M y c$ related to $H P R T$ in HEK293 cells transfected with different constructs. $13.8 N C$-GFP, Odf2 cloned into $p E G F P-N 1$ vector; $13.8 N C \triangle G F P$, Odf2 cloned into $p E G F P-N 1$ vector without $G F P ; p E G F P-1$, the $p E G F P$ vector without functional promoter; pEGFP-N1, the empty $p E G F P-N 1$ vector; shODF2, short hairpin Odf2 cloned into $p I R E S-G F P$ vector

\subsubsection{ODF2 over-expression affects the migration of human breast cancer cells}

Besides the role in canonical Wnt pathway $\beta$-catenin is also very important for cell-cell adhesion. $\beta$-catenin links cadherins at the plasma membrane to $\alpha$-catenin (Cowin et al., 1986; Ozawa et al., 1989; McCrea et al., 1991; Kemler et al., 1993; Takeichi et al., 1995). Therefore, the influence of over-expressed ODF2 on cell-cell adhesion was also investigated in NIH3T3, MCF7 and MDA-MB-231 cells using the wound healing assay.

To perform the assay, untransfected cells were used as control, cells stably transfected with empty vector $p E G F P-N 1$ as GFP control, and cells stably transfected with 13.8NC-GFP were applied to check the influence. In addition, the cell line NIH3T3 
was also investigated as control. The $100 \%$ confluent cells were scraped to form a wound and the distance between the borders of wound was measured at 0 hour (Fig. $3.27 \mathrm{~A})$. After certain times the distances between the borders of the wound were measured. Fig. 3.27 B shows that the NIH3T3 cells transfected with empty vector and with $13.8 N C-G F P$ have similarly moving speed, and both of them are faster than untransfected cells. This means that the alteration of cell migration might exclusively be caused by over-expressed GFP but not by ODF2 over-expression. Fig. 3.27 C shows that the MCF7 cells transfected with $13.8 N C$-GFP were distinctly affected by over-expressed ODF2 compared with untransfected cells and cells transfected with empty vector. The MCF7 cells transfected with $13.8 N C-G F P$ migrate faster. Surprisingly Fig. $3.27 \mathrm{D}$ shows that the effect of over-expressed ODF2 on cell migration in MDA-MB-231 cells is inversed compare to MCF7 cells. There is no distinct difference between untransfected cells and cells transfected with empty vector neither in MCF7 cells nor in MDA-MB-231 cells. Thereby GFP did not affect the migration of these two cell lines. These results demonstrate that over-expressed ODF2 has different effects on cell migration in different human breast cancer cell lines but no significant effect in NIH3T3 cells. 
A

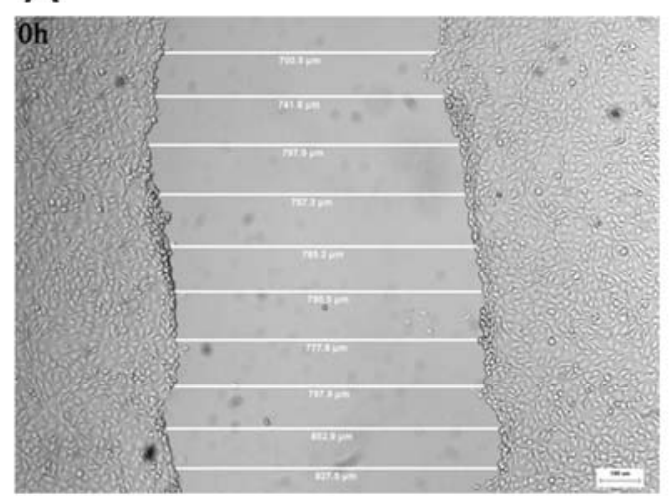

C

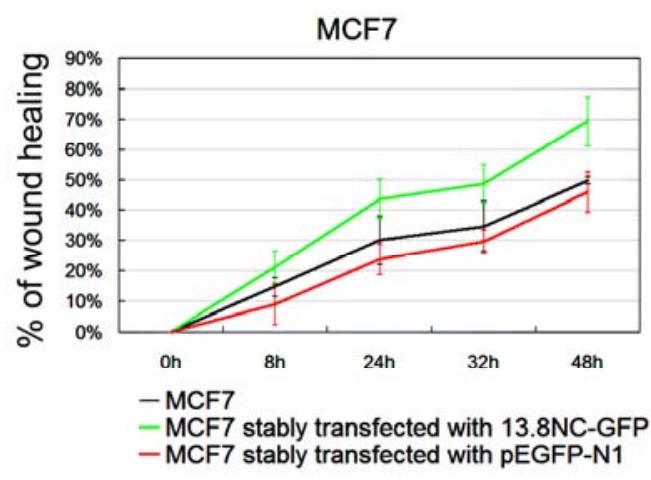

B

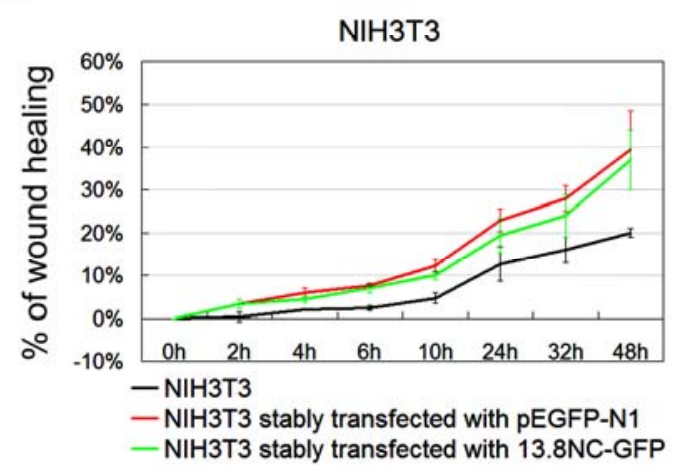

$\mathrm{D}$

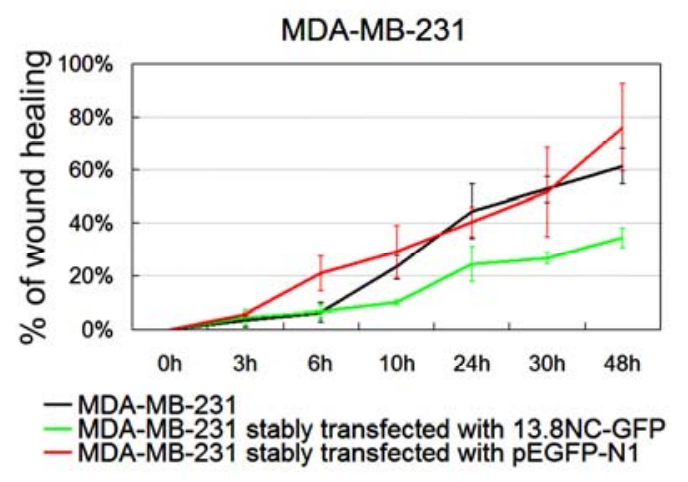

Fig. 3.27: Investigation of the effect of ODF2 over-expression on cell migration by wound healing assay in different cells. (A), an enlarged picture of NIH3T3 cells at $0 \mathrm{~h}$ as an example for wound healing assay. Each cell line was scratched at three different positions and each wound was measured at ten different positions. (B), (C), and (D), cell migration graphs. The curve with the larger percentage means that the cells migrate faster.

\subsubsection{ODF2 interacts with Axin1, Axin2 and TAZ proteins individually}

Axin1 and Axin2 are two functionally identical isoforms of Axin that is a scaffold protein cooperating with GSK3 $\beta$ and APC in mediating phosphorylation of $\beta$-catenin (Kikuchi, 1999; Chia and Costantini, 2005). Knock in of Axin2 into the deleted Axin1 gene rescues Axin1 $^{-/}$mice (Chia and Costantini, 2005). Moreover, it was demonstrated that Axin colocalized with ODF2 at the centrosome (Fumoto et al., 2009). Therefore it was predicted that ODF2 might bind to Axin and promote its 
stability thus affect the degradation of $\beta$-catenin. HEK293 cells were co-transfected with 13.8NC-GFP and Axin1-myc or with 13.8NC-GFP and Axin2-myc, respectively. Cells were lysed and soluble proteins were subjected to immunoprecipitation using anti-GFP antibodies which were raised in rabbit. The bead bound protein fractions were then analyzed by Western blot using polyclonal anti-Axin1 and anti-Axin2 antibodies, respectively. Axin1-myc (a doublet band at $\sim 120 \mathrm{kDa}-130 \mathrm{kDa}$ ) and Axin2-myc (at $\sim 100 \mathrm{kDa}$ ) were found to co-precipitate with 13.8NC-GFP fusion protein, respectively (Fig. 3.28 lane b in panel A and B, arrows on upper pictures). In contrast, no Axin1 or Axin2 was found in the control beads in which anti-GFP antibodies have been omitted (Fig. 3.28 lane bc in panel A and B). These results indicate that ODF2 might affect the stability of $\beta$-catenin negatively by binding to Axin1 and Axin2 directly.

It was reported that phosphorylated $\beta$-catenin serves as a scaffold for TAZ association with $\beta$-TrCP/E3 ubiquitin-ligase complex (Azzolin et al., 2012). As a transcriptional co-activator of the Hippo pathway that is a growth-limiting and differentiation-promoting signal TAZ promote cell proliferation and inhibit differentiation (Halder and Johnson, 2011; Hong and Guan, 2012; Ramos and Camargo, 2012). Hippo triggers phosphorylation and nuclear export of TAZ for degradation in the cytoplasm. Similar to phosphorylated $\beta$-catenin, TAZ is ubiquitinated and degraded by the E3 ligase $\beta$-TrCP (Murakami et al., 2005; MacDonald et al., 2009; Liu et al., 2010; Zhao et al., 2010; Imajo et al., 2012). Due to TAZ's association with many transcription factors via its interaction motifs (WW, coiled coil, SH3, and PDZ) (Hong and Guan, 2012), and the coiled-coil motifs of ODF2, it was investigated whether there is an interaction between TAZ and ODF2, and whether the effect of ODF2 on $\beta$-catenin is associated with TAZ. HEK293 cells were co-transfected with 13.8NC-GFP and TAZ-HA. Cells were lysed and soluble proteins were subjected to immunoprecipitation using anti-GFP antibodies which were raised in rabbit. The bead bound protein fractions were then analyzed by Western blot using monoclonal anti-HA antibodies. TAZ-HA $(\sim 55 \mathrm{kDa})$ was found to co-precipitate with 13.8NC-GFP fusion protein (Fig. $3.28 \mathrm{C}$, upper picture). In 
contrast, no TAZ was found in the control beads in which anti-GFP antibodies have been omitted (Fig. 3.28 lane bc in panel C). These results indicate that ODF2 might directly bind to TAZ.

A

Interaction of ODF2 and Axin1

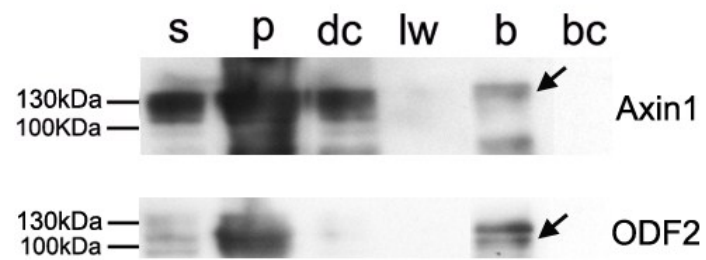

B

\section{Interaction of ODF2 and Axin2}

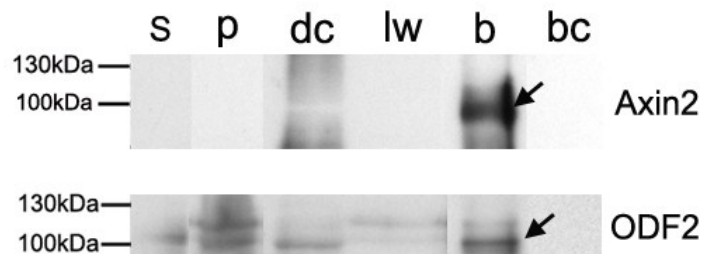

C

Interaction of ODF2 and TAZ

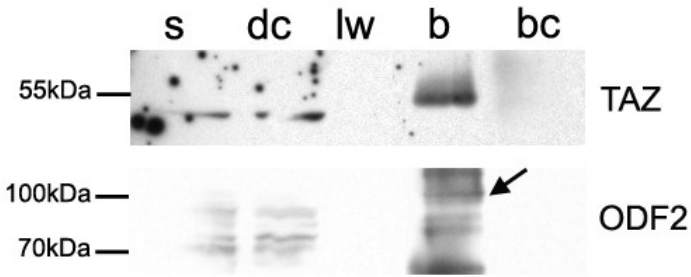

Fig. 3.28: Interaction of ODF2 and Axin1, Axin2, and TAZ, respectively. (A), interaction of ODF2 and Axin1. Plasmids encoding Axin1 fused to Myc (Axin1-myc) and ODF2 fused to EGFP (13.8NC-GFP) were transfected into HEK293 cells and co-immunoprecipitation was performed using anti-GFP antibodies. Axin1-myc (a doublet band at $\sim 120 \mathrm{kDa}-130 \mathrm{kDa}$ ) co-precipitated with 13.8NC-GFP detected using anti-Axin1 antibodies (arrow on upper picture). (B), interaction of ODF2 and Axin2. Plasmids encoding Axin2 fused to Myc (Axin2-myc) and ODF2 fused to EGFP (13.8NC-GFP) were transfected into HEK293 cells and co-immunoprecipitation was performed using anti-GFP antibodies. Axin2-myc ( 100kDa) co-precipitated with 13.8NC-GFP detected using anti-Axin2 antibodies (arrow on upper picture). (C), interaction of ODF2 and TAZ. Plasmids encoding TAZ fused to HA (Taz-HA) and ODF2 fused to EGFP (13.8NC-GFP) were transfected into HEK293 cells and co-immunoprecipitation was performed using anti-GFP antibodies. TAZ-HA $(\sim 55 \mathrm{kDa})$ co-precipitated with $13.8 \mathrm{NC}-\mathrm{GFP}$ detected using anti-HA antibodies. In all panels, ODF2 was proven to be ectopically expressed ( 100kDa, arrows on 
lower pictures) with anti-GFP antibodies. s, soluble cellular proteins; $p$, insoluble cellular proteins; $\mathrm{dc}$, proteins of the supernatants after separation of protein $\mathrm{G}$ agarose beads (depletion control); lw, proteins of the last washing step; $b$, proteins of the bead bound fraction. bc, control, proteins bound to beads without the presence of the fishing antibody. These three co-immunoprecipitations were performed by bachelor student Constanza Tapia.

\subsubsection{Ectopically expressed ODF2 affects the phosphorylation of Tau}

ODF2 is a self-interacting, microtubule-associated protein (Donkor et al., 2004). It is thus possible that the positive influence of ODF2 on $\beta$-catenin degradation is mediated by stabilizing microtubules subsequently maintaining $\beta$-catenin destructure complex. Therefore I investigated the effect of ectopically expressed ODF2 on another microtubule-associated protein Tau. As a microtubule-associated protein, Tau stabilizes microtubule polymers (Brandt and Lee, 1993) and suppresses microtubule dynamics (Panda et al., 1995). Normally, phosphorylation of the microtubule-associated proteins by the microtubule-affinity-regulating-kinase causes the microtubule-associated proteins to detach from any bound microtubules (Drewes et al., 1998), and the hyperphosphorylation of Tau leads to massive detachment, which in turn greatly reduces the stability of microtubules in nerve cells (Mandelkow and Mandelkow, 1995). The NIH3T3 cells were transfected with Odf2 siRNA leading a reduced expression of ODF2 (Fig. 3.29 A) and the HEK293 cells were transfected with 13.8NC-GFP causing an over-expression of ODF2 (Fig. 3.29 C). After 48 hours of Odf2 siRNA transfection and 24 hours of ODF2 over-expression, the cells were lysed and analysed with Western blotting. Relatively quantified results show, that Tau proteins were more phosphorylated when ODF2 was less expressed, and consistently, Tau proteins were less phosphorylated when ODF2 was over-expressed (Fig. 3.29 B and D). These results indicate that ODF2 promotes the stabilization of microtubules and might therefore abet the degradation of $\beta$-catenin. 
A

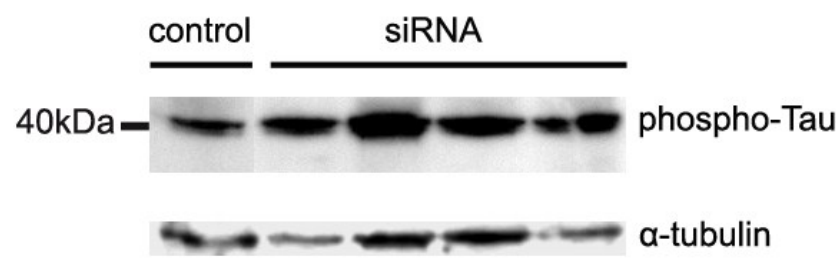

B

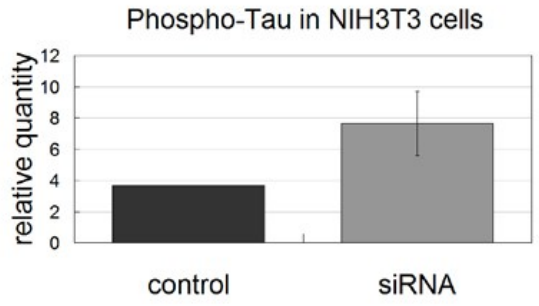

C

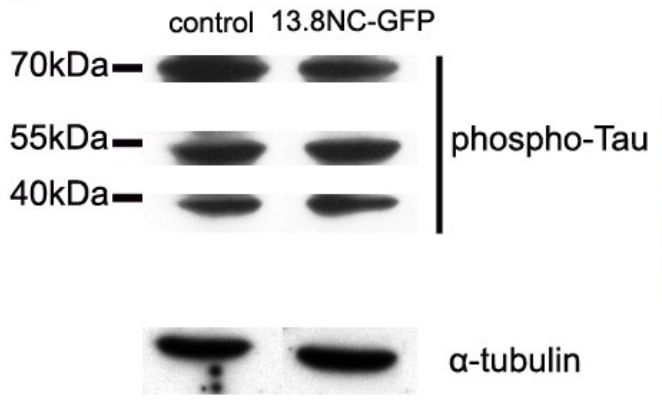

D

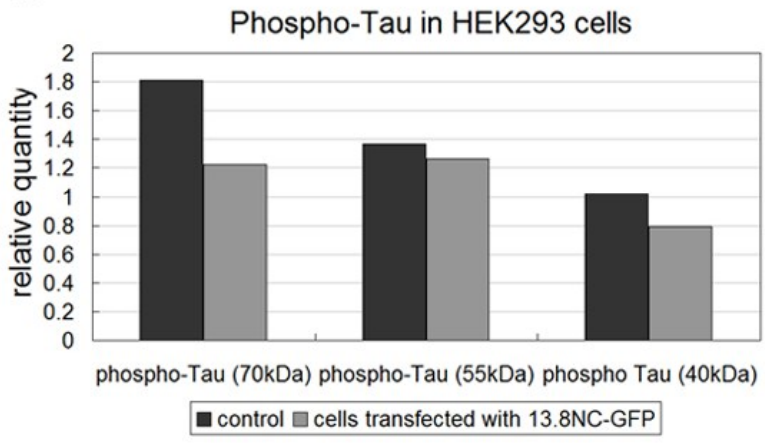

Fig. 3.29: The phosphorylation of Tau was affected by ectopically expressed ODF2. (A), the phosphorylation of Tau increases in NIH3T3 cells in that Odf2 was knocked down. After using small interfering RNA the soluble proteins were separated on SDS-gels, blotted, and probed with anti-phospho-Tau antibodies. Odf2 expression was interfered by small interfering RNA constructs, and four independent experiments were performed. The untreated NIH3T3 cells were taken as control. In NIH3T3 cells, many different degraded phospho-Tau fragments were found. $\alpha$-tubulin was taken as reference protein. (B), the relative quantitative results of main degraded phospho-Tau expression $(\sim 40 \mathrm{kDa})$ normalized to $\alpha$-tubulin according to Western blot result in panel A. (C), the phosphorylation of Tau decreases in ODF2 over-expressed HEK293 cells. Plasmids encoding ODF2 fused to EGFP (13.8NC-GFP) were transfected into HEK293 cells, afterwards, the soluble proteins were separated on SDS-gels, blotted, and probed with anti-phospho-Tau antibodies. The untreated HEK293 cells were taken as control. In HEK293 cells, many different degraded phospho-Tau fragments were found. $\alpha$-tubulin was taken as reference protein. (D), the relative quantitative results of three phospho-Tau degradation fragments $(\sim 70 \mathrm{kDa}, \sim 55 \mathrm{kDa}$ and $\sim 40 \mathrm{kDa}$, respectively) normalized to $\alpha$-tubulin according to Western blot result in panel A. 


\section{Discussion}

\subsection{ODF1 is essential for tight linkage of sperm head to tail and male fertility in mice}

Up to $15 \%$ of reproductive-aged couples worldwide suffer from infertility according to the World Health Organization. In $50 \%$ of cases male infertility has been found to be the cause (Cui, 2010). In male infertility diagnostics, the sperm tail cytoskeleton has received increasing attention over the years since sperm tail-associated defects may impair sperm motility that eventually results in infertility (Marmor and Grob-Menendez, 1991). Likewise, sperm tail defects have been considered as a prognostic factor in assisted conception in humans (Lim et al., 1998).

The sperm tail generates progressive motility necessary to reach the oocyte. Its functional unit is the axoneme, a tubulin-based structure containing microtubule-associated proteins and motor proteins that convert chemically bound energy into progressive mechanical force by ATP hydrolysis (Haimo and Rosenbaum, 1981; King, 2000; Mortimer, 1997; Porter and Sale, 2000). The orderly array of axonemal nine doublet microtubules surrounding a pair of singlet microtubules is complemented by associated ODFs that accompany the microtubule doublets throughout most of the sperm tail. In addition, in the midpiece of the sperm tail the mitochondrial sheath wraps around the ODFs, and in the principal piece the fibrous sheath surrounds the axoneme and the residual seven ODFs (Fawcett, 1975). Both the ODFs and the fibrous sheath provide structural rigidity to the tail (Baltz et al., 1990). The fibrous sheath additionally is involved in the generation of ATP (Miki et al., 2004). A main protein component of mammalian ODFs is ODF1. Moreover, ODF1 is exclusively expressed in spermatids and restricted to the ODF compartment (Morales et al., 1994). Not surprisingly, it was observed that Odfl-deficiency did not affect female mice. Loss of ODF1 exclusively affected male mice resulting in infertility.

In testes it was found that spermatogenesis in general was not impaired. The expression of all marker genes characteristic for the various germ cell types 
demonstrated that spermatogenesis proceeded normally from spermatogonia to spermatids. Expression of Hanpl especially substantiated spermiogenic progression in Odf1-deficient mice because Hanpl transcription is restricted to spermatids (Tanaka et al., 2005). Histological examinations of testes likewise revealed no hints of disturbed spermatogenesis up to at least 19 days after birth. Perturbations of the germinal epithelium were found not until sexual maturity when two-thirds of mice exhibited undifferentiated cells, and two of nine mice showed multinuclear cells in the lumen of their seminiferous tubules. However, in most cases elongated spermatids were present.

Semithin sections of epididymides from $\mathrm{Odfl}^{-/-}$mice revealed an increase of nonmature germ cells and a high percentage of dysplastic sperm. Sperm heads were barely found, thus accounting for observed infertility. The presence of large multinucleate cells found in some tubules suggests that the lack of ODF1 also might affect germ cell separation, leading eventually to the coalescence of adjacent germ cells. Measurement of sperm parameters revealed that Odfl-deficient epididymal sperm are motile, although with strongly reduced velocities but increased beat cross frequencies and straightness, both of which might be caused by the absence of the normally built sperm head. In addition, half-reduction of the amount of ODF1 in sperm of heterozygous mice, originating from the syncytial nature of the germinal epithelium (Braun et al., 1989), affected their motility but eventually did not impact fecundity. At the light microscopic level, Odfl-deficient sperm are characterized by their remarkable tail coiling, as well as by their missing heads. At the ultra-structural level, disturbed mitochondrial organization in the midpiece of the sperm tail and disturbed organization of ODFs not only in the midpiece but also in the principal piece were observed. Most remarkably, however, is the absence of intact spermatozoa. These results thus strongly support the view that ODF1 is essential for the tight connection of the sperm head to the tail and for the orderly arrangement of the mitochondria and the ODFs in the sperm tail.

Besides the observation of mitochondrial disorganization in $O d f 1^{-/-}$mice, loss of either Nectin-2, a $\mathrm{Ca}^{2+}$-independent immunoglobulin-like cell-cell adhesion molecule, or 
Gopc (Golgi-associated PDZ- and coiled-coil motif-containing protein) showed impairment of tight packaging of mitochondria into a helical sheath (Bouchard et al., 2000; Suzuki-Toyota et al., 2007). However, neither Nectin-2 nor Gopc have previously been identified as ODF1 binding partners. Instead, a promising candidate that could be responsible for mediating the tight packaging of mitochondria and their association to the ODFs in the midpiece of the sperm tail is the kinesin light chain, KLC3. KLC3 has not only been shown to interact with ODF1 but also to associate with mitochondria in the sperm tail (Bhullar et al., 2003; Zhang et al., 2004). Lack of its binding partner ODF1 could thus affect the tight mitochondrial association to the ODFs eventually resulting in its disturbed arrangement.

It has also been found that the ODFs associate to the axonemal microtubule doublets no more tightly in midpiece and principal piece in $O d f 1^{-/-}$mice. Lack of ODF1 seems to result in reduction or even loss of the central part of ODF, the medulla, and eventually in hollow fibers (Fig.3.7 B b). Beyond that, the most striking phenotype was the detachment of the tail from the nucleus. It resembles a type of oligoteratoasthenospermia in humans with sperm tail fragility and decapitated sperm heads (Baccetti et al., 2001; Chemes et al., 1999; Kamal et al., 1999). Detachment might occur during sperm passage from testis to epididymidis due to the loosening of the sperm head-to-tail linkage by missing ODF1 that eventually could not withstand mechanical forces.

Whereas the heterozygous male mice, that were generated from mixed genetic background (C57BL/6//129/Sv), are fully fertile albeit having reduced sperm motility, the incipient congenic heterozygous male mice, generated by successive backcrossing of Odfl-depleted mice of mixed background (either heterozygous females or males) to inbred $129 / \mathrm{Sv}$ mice, are strongly reduced in fertility. In six individual matings, incipient congenic male mice of backcross generation N7 gave birth to only two pups after more than 5 months of continuous cohabitation. Moreover, male fertility rate is already reduced to $\sim 50 \%$ in backcross matings compared to wild-type crosses. Whereas fertility of heterozygous females continuously drops during progressive 
backcrosses, male fertility is already severely reduced in generation N0. The decline in female fertility by successive backcrossing to $129 / \mathrm{Sv}$ suggests that the genetic background strongly affects fertility success. However, severe subfertility of male mice could not be explained exclusively by the genetic background since heterozygous females of generation N7 gave birth to 3 pups per litter when mated to wild-type males (Fig. 3.8 B). Both together, severe subfertility of heterozygous male mice and reduced fertility of female mice might account for the infertility observed in generation N7 pairings. The generally much higher litter size of strain C57BL/6 might explain why haplo-deficiency of ODF1 seemed not to affect male fertility on mixed genetic background (C57BL/6//129/Sv).

Although normal sperm count, sperm motility and no ultra-structural sperm anomalies of congenic heterozygous males were detected, a significant increase of the distance between the nuclear membrane and the capitulum was yet observed, when ODF1 is reduced. A larger distance between nucleus and capitulum reflects a weakening of sperm head-tail linkage that eventually causes decapitation when strong shearing forces are applied as e.g. during sperm movement through the female genital tract.

The impact of ODF1 on the structural organization of the spermatozoon is illustrated in Fig. 4.1, emphasizing known interaction partners. Linking ODFs to microtubules might occur via ODF1/SPAG5/SPAG4 interaction and to mitochondria via ODF1/KLC3 interaction. ODF1/ODF2 binding might account for rigidity of ODFs, basal plate, capitulum, and striated columns (Schalles et al., 1998). The results of ODF1 investigation suggest that ODF1 is essential for the rigid junction of sperm head and tail and that a loss of function of ODF1 might account for some of the cases of human infertility with decapitated sperm heads. 


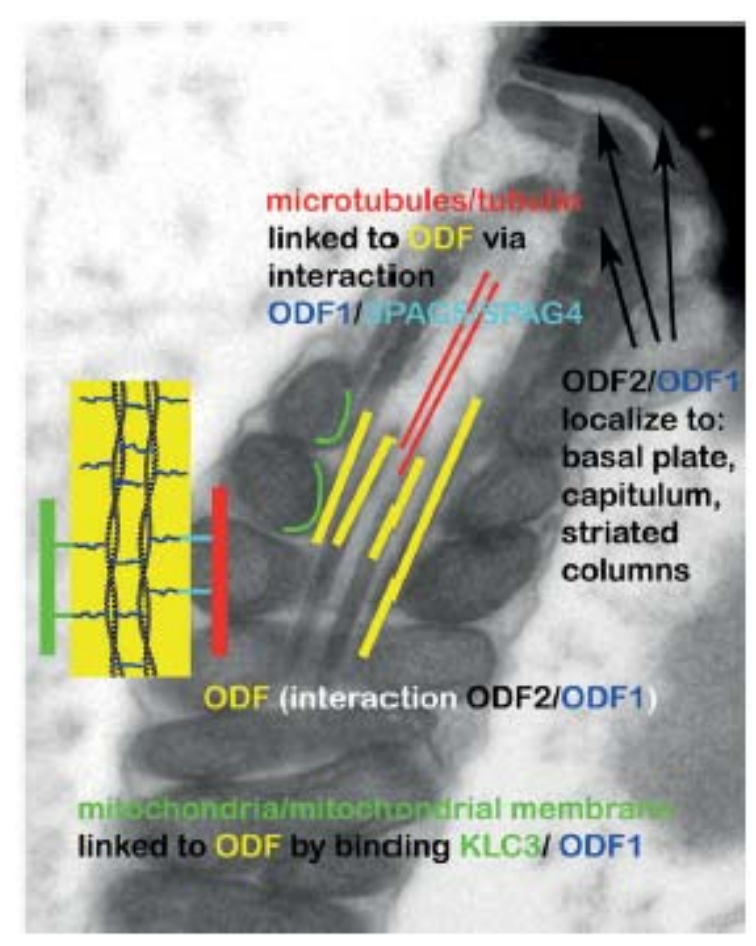

Fig. 4.1: The localization of ODF1 and its reported interaction partners that might account for observed disturbances in ODF1-deficient sperm. ODF1 interacts with ODF2, and both are located to the ODFs, basal plate, capitulum, and striated columns (Schalles et al., 1998). Linking of ODF to axonemal microtubules might be mediated via binding of ODF1 to SPAG5/SPAG4 and of ODF to mitochondria via KLC3 binding.

\subsection{Interaction of ODF1 and SPAG4 defines a novel germ cell specific LINC complex involved in sperm head to tail attachment}

The most striking phenotype observed in Odfl knock out mice was sperm decapitation causing male infertility. ODF1, therefore, has been turned out to be an important component of the head-to-tail coupling apparatus (HTCA) in sperm (see 3.1 and Yang et al., 2012). ODF1 most likely ensures the connection to capitulum and sperm tail by interaction with ODF2 that is a fiber forming protein and the main component of the sperm tail outer dense fibers (Shao et al., 1997; Petersen et al., 1999). ODF1 thus belongs to the cytoskeletal compartment of the HTCA. But which protein (or proteins) 
might then be responsible for mediating the tight connection of the capitulum to the nuclear membrane by binding to ODF1? Presumably, the respective counterpart has to be expressed in male germ cells and preferentially localized to the nuclear membrane. In somatic cells the cytoskeleton is linked to the nucleoskeleton by nuclear membrane proteins with conserved SUN or KASH domains. SUN domain proteins with predominant expression in testis have already been identified although meticulous analyses are still missing. One of these proteins is SPAG4. SPAG4 has been described as an interaction partner of ODF1 that localizes to the microtubules of manchette and axoneme (Shao et al., 1999). However, albeit proven by immunoelectron microscopy the cytoskeletal localization is quite uncommon for SUN domain proteins that are usually nuclear membrane proteins. I therefore started to investigate expression and localization of SPAG4 in depth. Full-length Spag4 was cloned from mouse testis. The protein consists of the N-terminal region followed by the putative transmembrane domain, a coiled-coil domain and the conserved SUN domain and thus revealed its affiliation to the SUN domain protein family. Its domain organization additionally indicated a membranous association. I could indeed show that SPAG4 is a nuclear membrane protein just as other SUN domain proteins. When ectopically over-expressed a conspicuous amount of SPAG4 is cytoplasmic although no hints to microtubule association were found. In vivo proteinase $\mathrm{K}$ treatment demonstrated that the C-terminal region is more resistant against proteolytic degradation demonstrated by the fact that only antibodies directed against the C-terminally located Myc-tag were able to detect the full-length protein as well as a resistant fragment of $\sim 40 \mathrm{kDa}$. These data thus indicate that SPAG4 is located in the nuclear membrane with its C-terminal region most likely residing in between the outer and inner nuclear membrane. The Spag4 gene is exclusively expressed in testis. Expression was found neither in somatic tissues nor in ovary. Additionally, spermatogonial stem cells (SSC), embryonic stem cells (ES) or the teratocarcinoma cell line F9 did not express Spag4. Transcription of Spag4 in testis starts around day 20 to 25 in mice which corresponds to the emergence of the early round spermatid stage. Transcription of Spag4 therefore seems to be restricted to the male germ cell lineage starting at the round spermatid 
stage. Immunocytology on testicular cell preparations confirmed previous data. SPAG4 protein was identified exclusively in male germ cells from round spermatids to late elongating spermatids. The protein was always found at the nuclear membrane and became concentrated towards the posterior pole of the spermatids during spermiogenesis. SPAG4 was located in proximity to the manchette but did not show exact co-localization. When the manchette started to disintegrate a specific amount of SPAG4 scattered into the residual cytoplasm. In mature sperm SPAG4 was no longer detectable most likely due to its amount below the detection limit. I could never verify SPAG4 in the sperm tail. However, it might be possible that immunoelectron microscopy is even more sensitive to enable detection of the protein in the sperm tail and the manchette (Shao et al., 1999). Eventually, these results demonstrate that SPAG4 is a nuclear membrane protein restricted to haploid male germ cells and localized to the posterior pole of spermatids - a region in which the sperm tail attaches to the nucleus. Furthermore, I verified that SPAG4 interacts with ODF1 endogenously in testis as well as when ectopically over-expressed. Interaction was proven for both the N-terminal fragment of SPAG4 including the transmembrane domain as well as the C-terminal part. Interaction with ODF1 was demonstrated by co-IP as well as by immunocytology. The C-terminal fragment missed the transmembrane domain and therefore displayed an aberrant cytoplasmic localization. The N-terminal fragment comprising the transmembrane domain localized to the nuclear membrane and caused the recruitment of ODF1 to the nuclear membrane as well as was also shown for the full length SPAG4 protein. These results therefore suggest that the nuclear membrane protein SPAG4 most likely recruits ODF1 to the nuclear membrane via its N-terminal end that resides in the cytoplasm. Location of SPAG4, vice versa, is not provoked by ODF1. However, I have to admit that cytoplasmic location of the N-terminal end of the SUN domain protein SPAG4 is quite uncommon for a member of this protein family. Beyond, by ectopic over-expression of ODF1 I found that ODF1 is concentrated at the centrosome, which was identified by $\gamma$-tubulin staining. When simultaneously over-expressed, SPAG4 is also concentrated at the centrosomal area. Since the centrosome comprises ODF2 and can be transformed into the basal body to 
grow a cilium it could be viewed as an equivalent to the connecting piece in sperm: via binding to ODF2 ODF1 is recruited to the centrosome/connecting piece and via interaction ODF1-SPAG4 the centrosome/connecting piece is linked to the nuclear membrane (Yang et al., in preparation). My data therefore suggest that the interaction between ODF1 (in the capitulum) and SPAG4 (in the nuclear membrane at the implantation fossa) is the molecular equivalent of the HTCA. The interaction of ODF1 and SPAG4 is illustrated in Fig. 4.2. This model is supported by the fact that the presumed Drosophila ortholog of SPAG4 is likewise required for the attachment of the basal body to the spermatid nucleus (Kracklauer et al., 2010). However, the final proof will be attained by the generation of Spag4 deficient mice and their observed phenotype. In conclusion, it is suggested that SPAG4-ODF1 interaction defines a novel LINC complex in spermatids that mediates the attachment of the sperm head to the tail.

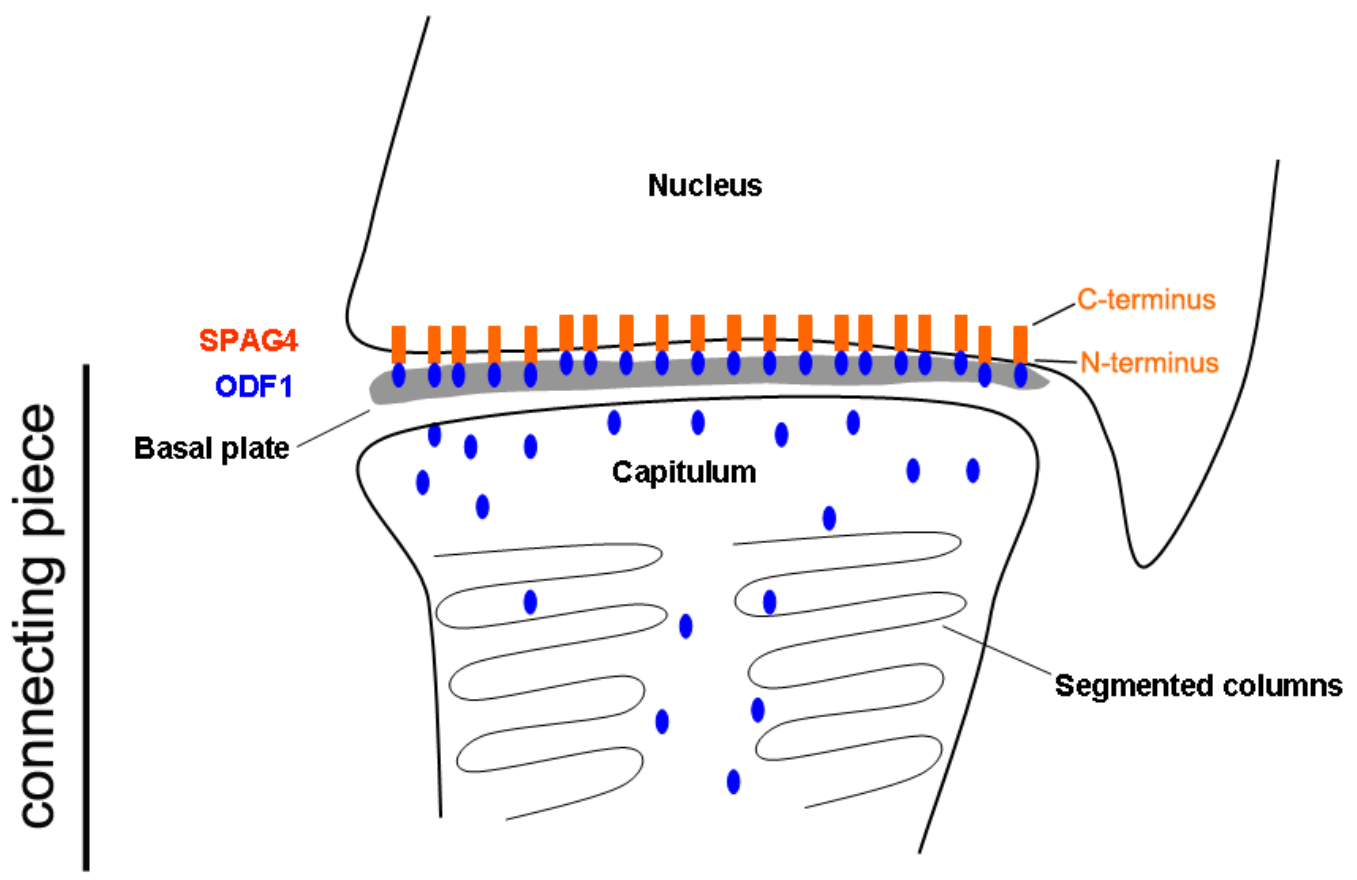

Fig. 4.2: The interaction between ODF1 and SPAG4 defines a novel LINC complex in spermatids that mediates the attachment of the sperm head to the tail. ODF1, that locates at the basal plate of the connecting piece of spermatids, is linked to the nuclear membrane via the N-terminal end of SPAG4. 


\subsection{ODF2 positively affects degradation of $\beta$-catenin due to influencing its destruction complex}

Whereas ODF1 is exclusively expressed in spermatids and restricted to the ODF compartment (Morales et al., 1994), ODF2 is not only expressed in male germ cells (Brohmann et al., 1997; Shao et al., 1997; Turner et al., 1997; Hoyer-Fender et al., 1998; Schalles et al., 1998; Petersen et al., 1999) but also localizes generally to the appendages of the mother centriole in somatic cells (Nakagawa et al., 2001; Hoyer-Fender et al., 2003; Donkor et al., 2004). Ishikawa's group reported that the Odf2 gene is essential for the formation of distal/subdistal appendages and the generation of primary cilia. Using immunofluorescence and ultrathin-section electron microscopy, they showed that in $O d f 2^{-/}$F9 cells, distal/subdistal appendages disappear from mother centrioles, and the formation of primary cilia is completely suppressed (Ischikawa et al., 2005). In recent years, it has been found that primary cilia are involved in several signaling pathways essential for growth and differentiation, including the Hedgehog (Hh), Wnt, and PDGF pathways (Eggenschwiler and Anderson, 2007; Berbari et al., 2009). Furthermore, many components (Dvl, Inversin, APC, GSK3 $\beta$, Axin1, Axin2, and $\beta$-catenin) of canonical Wnt pathway have been detected to localize to the centrosome, and some of them take part in regulating centrosomal functions (Kaplan et al., 2004; Louie et al., 2004; Fumoto et al., 2006; Hadjihannas et al., 2006; Huang et al., 2007; Corbit et al., 2008; Bahmanyar et al., 2008; Park et al., 2008; Kim et al, 2009; Fumoto et al., 2009). Correspondingly, basal bodies and primary cilia simultaneously regulate both canonical and non-canonical Wnt pathways (Simons et al., 2005; Gerdes et al., 2007; Kishimoto et al., 2008; Corbit et al., 2008; reviewed in Bisgrove and Yost, 2006). Due to the tight association of the canonical Wnt pathway and all three organelles (centrosomes, basal bodies and primary cilia), the influence of centrosomal protein ODF2 on canonical Wnt pathway was investigated.

Former colleagues of our group analysed many possible interactions between ODF2 and components of the canonical Wnt pathway, among them, using pull-down and 
co-immunoprecipitation it has been detected that ODF2 interacts with $\beta$-catenin. Therefore, I was focusing on the effect of ODF2 on the canonical Wnt key protein, $\beta$-catenin. Using reporter gene assays in cultured cells I found that over-expression of ODF2 negatively affects the canonical Wnt signaling. In addition, the result of a quantitative Western blot analysis shows that over-expressed ODF2 could conspicuously abet the degradation of over-expressed $\beta$-catenin. In fact, not only the degradation of over-expressed $\beta$-catenin was affected, when ODF2 over-expression level is extremely high, the degradation of endogenous $\beta$-catenin can also be promoted. Furthermore, the transcription level of a Wnt target gene, $c-M y c$, was reduced by the over-expression of ODF2. In support of these results, a microinjection assay was performed in Xenopus embryos. This result shows that over-expressed ODF2 moderately inhibited Xwnt-8 induced secondary axis formation in Xenopus embryos. Altogether, these results suggest that ODF2 inhibits the canonical Wnt pathway possibly due to promoting the degradation of $\beta$-catenin.

ODF2 protein domains, which might be responsible for canonical Wnt pathway inhibition, were investigated. The cenexin insertion in the $\mathrm{N}$-terminal region as well as an overall coiled-coil structure in the $\mathrm{C}$-terminal region showed a strong repression on the canonical Wnt pathway. So far, no enzymatic function of ODF2 has been found, and based on its overall coiled-coil domain, ODF2 is most likely a structural protein. This view is consistent with the result of a former colleague of our group, that ODF2 is a self-interacting, microtubule-associated protein (Donkor et al., 2004). In my work, I found that ODF2 interacts with Axin1 and Axin2. Axin1 and Axin2 are isoforms of Axin that is a scaffold protein cooperating with GSK3 $\beta$ and APC mediating phosphorylation of $\beta$-catenin (Kikuchi, 1999; Chia and Costantini, 2005). Therefore, due to interactions between ODF2 and $\beta$-catenin, Axin1 and Axin2 each, it seems that ODF2 takes part of the scaffolding protein complex and stabilizes bound $\beta$-catenin, resulting in an increased degradation of $\beta$-catenin. Inhibition of GSK3 $\beta$ by lithium increased $\beta$-catenin-dependent activation of a reporter vector. However, activation of the reporter in the presence of over-expressed ODF2 was not fully restored by lithium suggesting that a part of $\beta$-catenin might be bound to ODF2, 
therefore this part of $\beta$-catenin could not function as transcriptional activator. In mitosis, $\beta$-catenin localizes to centrosomes, and deletion of $\beta$-catenin results in monopolar spindles with duplicated but unseparated centrosomes (Kaplan et al., 2004). Consistently, stabilization of $\beta$-catenin increases the distance between centrosomes in interphase cells and between centrioles in G1/S phase of the cell cycle (Bahmanyar et al., 2007). Additionally, Axin colocalizes with ODF2 to centrosome (Fumoto et al., 2009). Therefore, the scaffolding protein complex of ODF2 and Axin might be involved in the regulation of centrosome cohesion via promoting the degradation of $\beta$-catenin.

A microtubule-associated protein Tau was less phosphorylated in the presence of over-expressed ODF2 and vice versa pointing to a function of ODF2 by stabilization of microtubule. It seems that ODF2 stabilizes the destruction complex by linking scaffolding proteins Axin and $\beta$-catenin, further by binding this destruction complex to microtubules. Interference of microtubule polymerization by nocodazole increased $\beta$-catenin-dependent activation of a reporter vector as well as taxol-mediated over-stabilization of microtubules. Moreover, activity of the canonical Wnt pathway could be down-regulated by centrosomal and cytoskeletal proteins. This result suggests that the canonical Wnt pathway tightly associates with centrosome and microtubules.

It was reported that hSav1-Mst2-Nek2A complex including two Hippo pathway components, the mammalian sterile 20-like kinase 2 (Mst2) and the scaffold protein Salvador (hSav1), functions in centrosome disjunction (Mardin et al., 2010). In my work, I found that ODF2 interacted with TAZ, a major transcriptional co-activator of the Hippo pathway, ubiquitinated by the E3 ligase $\beta$-TrCP mediated by phosphorylated $\beta$-catenin as its scaffold (Murakami et al., 2005; MacDonald et al., 2009; Liu et al., 2010; Zhao et al., 2010; Imajo et al., 2012; Azzolin et al., 2012). This finding suggests that like the canonical Wnt pathway, more and more components of the Hippo pathway are involved in regulating functions of centrosome.

As a multifunctional protein, besides the roles in canonical Wnt pathway (Wieschaus et al., 1984) and the centrosome cycle (Kaplan et al., 2004), $\beta$-catenin also plays an 
important role in cell-cell adhesion (Ozawa et al., 1989). When canonical Wnt pathway is inactivated, $\beta$-catenin locates at the cytoplasmic side of the membrane as a component of cadherin-based cell-cell connections. Using MCF7, a human breast cancer cell line retaining characteristics particular to the mammary epithelium, I found that cells migrated faster in the presence of over-expressed ODF2. The increasing migration speed of cells was most likely induced by an instable cell-cell connection probably mediated by reduced $\beta$-catenin. This view supports the conclusion that over-expressed ODF2 promotes the degradation of $\beta$-catenin. However, in two further cell lines, NIH3T3 and MDA-MB-231, the results were not consistent with those in MCF7. In NIH3T3 cells, over-expressed ODF2 has no significant effect on cell migration. In MDA-MB-231 cells, a human breast cancer cell line retaining mesenchymal-like phenotype, cells migrated slower in the presence of over-expressed ODF2. Because mesenchymal cells lose their cell-cell adhesion, reduced migration speed of MDA-MB-231 cells could not be caused by influencing the degradation of $\beta$-catenin by over-expressed ODF2. The reason for the effect of ODF2 on cell migration in MDA-MB-231 is still unknown.

Taken all together, besides the structural function of ODF2 in ciliogenesis, ODF2 inhibits the canonical Wnt pathway probably due to promoting the degradation of $\beta$-catenin. The increase of degradation of $\beta$-catenin caused by ODF2 is possibly mediated by stabilizing $\beta$-catenin bound to a scaffolding protein complex including ODF2 and Axin and further by binding this destruction complex to microtubules. In addition, ODF2 is predicted to contain overall CKII and PKC phospho-sites (http://myhits.isb-sib.ch/cgi-bin/motif_scan). CKII positively regulates the canonical Wnt pathway via phosphorylation of $\beta$-catenin at its $\mathrm{Thr}^{393}$, leading to proteasome resistance and increased protein and co-transcriptional activity (Song et al., 2003). Therefore, ODF2 might also be an antagonist of $\beta$-catenin through competing CKII to promote the degradation of $\beta$-catenin. 


\section{List of figures and tables}

Fig. 1.1: Spermatogenesis.

Fig. 1.2: Schema drawing of spermiogenesis, here shown for human.........................7

Fig. 1.3: The Spermatozoon...........................................................................8

Fig. 1.4: Enlargement of connecting piece of mouse sperm tail............................10

Fig. 1.5: Stages of spermatogenesis and steps of spermiogenesis in mice (Russell et al., 1990)

Fig. 1.6: Centrosome, primary cilium and cell cycle............................................16

Fig. 1.7: Wnt/ $\beta$-catenin signalling pathway............................................19

Fig. 1.8: The faces of $\beta$-catenin within the cell.................................................20

Fig. 3.1: Targeted disruption of the promoter region and of exon 1 of the Odf1 gene.......51

Fig. 3.2: Testicular histology of heterozygous and homozygous $O d f 1$-deficient mice..........55

Fig. 3.3: Marker gene expression revealed spermatogenic progression irrespective of the

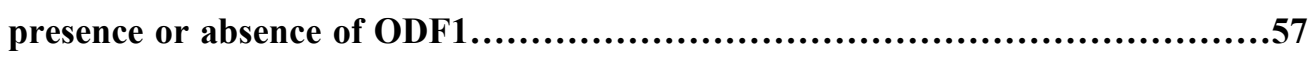

Fig. 3.4: Semithin sections of wild-type and homozygous Odf1-deficient mouse testes and epididymides.......................................................................57

Fig. 3.5: Computer-assisted analysis of sperm motility.....................................59

Fig. 3.6: Acrosome and axoneme in Odf1-mutant sperm.......................................60

Fig. 3.7: Ultrastructure of spermatozoa....................................................63

Fig. 3.8: Impaired fertility of congenic heterozygous $O d f 1$-deficient males....................65

Fig. 3.9: Computer-assisted analysis of sperm motility.....................................67

Fig. 3.10: In vitro fertilization capability of wild-type $129 / \mathrm{Sv}$ and congenic heterozygous odf1 $1^{+/-}$sperm.

Fig. 3.11: Ultra-structural analyses of spermatozoa from incipient congenic heterozygous Odf1 $^{+-}$mice.

Fig. 3.12: Amino acid sequence alignment and domain organization of SPAG4 and

SPAG4L-2 from Mus musculus..................................................73

Fig. 3.13: Spag4 is specifically transcribed in male germ cells.................................74

Fig. 3.14: Ectopic expression of SPAG4 in HEK293 cells revealed nuclear membrane

Localization..................................................................77

Fig. 3.15: The N-terminal end of SPAG4 is prone to in situ proteinase $\mathrm{K}$ digestion...........78

Fig. 3.16: Confirmation of SPAG4 self-interaction by co-immunoprecipitation...............79

Fig. 3.17: Endogenous expression of SPAG4 in spermatids...............................80

Fig. 3.18: Interaction of ODF1 and SPAG4 ..............................................83

Fig. 3.19: Redistribution of SPAG4 towards the posterior pole of elongating spermatids is not mediated by ODF1................................................................85

Fig. 3.20: Ectopically expressed ODF1 located at the centrosome and is recruited to the nuclear membrane by SPAG4 (full length, flSPAG4) and its N-terminal region ..............................................................................87

Fig. 3.21: The influence of over-expressed ODF2 on $\beta$-catenin activity.........................89

Fig. 3.22: Relative quantity of $\beta$-catenin in HEK293 cells treated with cycloheximide.........92

Fig. 3.23: The influence of different ODF2 protein domains translated from a series of 
deletion constructs on canonical Wnt pathway

Fig. 3.24: Analysis of the influence of centrosomal and cytoskeletal proteins on canonical Wnt reporter. .97

Fig. 3.25: The comparison of single injection of Xwnt-8 (8pg) mRNA and the co-injection of Xwnt-8 (8pg) and different amounts of Odf2 mRNA (50pg, 100pg and 200pg, respectively) on secondary axis formation (cooperation with Dr. B. Rust, Henningfeld's group).

Fig. 3.26: Transcription of $c-M y c$ related to HPRT in HEK293 cells transfected with different constructs.

Fig. 3.27: Investigation of the effect of ODF2 over-expression on cell migration by wound healing assay in different cells. 103

Fig. 3.28: Interaction of ODF2 and Axin1, Axin2, and TAZ, respectively. .105

Fig. 3.29: The phosphorylation of Tau was affected by ectopically expressed ODF2. .107

Fig. 4.1: The localization of ODF1 and its reported interaction partners that might account for observed disturbances in ODF1-deficient sperm. 112

Fig. 4.2: The interaction between ODF1 and SPAG4 defines a novel LINC complex in spermatids that mediates the attachment of the sperm head to the tail.

Tab. 2.1: Vectors...............................................................................22

Tab. 2.2: Oligonucleotides.................................................................23

Tab. 2.3: Enzymes..........................................................................24

Tab. 2.4: Primary antibodies.................................................................25

Tab. 2.5: Secondary antibodies...........................................................25

Tab. 2.6: Culture media for $E$. coli cultures..................................................26

Tab. 2.7: Culture media for eukaryotic cell culture........................................26

Tab. 2.8: Buffers and solutions.......................................................27

Tab. 2.9: Chenicals........................................................................29

Tab. 2.10: Kits..................................................................................31

Tab. 2.11: G418 concentrations of different cell culture media................................38

Tab. 2.12: Primers application of RT-PCR in Odf1 knock out mice..........................42

Tab. 3.1: Fertility, fecundity, and testis weight of wild-type and $O d f 1$ mutant mice...........53 


\section{References}

Adham, I. M., Burkhardt, E., Benahmed, M., \& Engel, W. (1993). Cloning of a cDNA for a novel insulin-like peptide of the testicular Leydig cells. Journal of Biological Chemistry, 268(35), 26668-26672.

Austin, C. (1951). Observations on the penetration of the sperm into the mammalian egg. Australian Journal of Biological Sciences, 4(4), 581-596.

Azzolin, L., Zanconato, F., Bresolin, S., Forcato, M., Basso, G., Bicciato, S., Cordenonsi, M., \& Piccolo, S. (2012). Role of TAZ as mediator of Wnt signaling. Cell,151(7), 1443-1456.

Baccetti, B., Capitani, S., Collodel, G., Di Cairano, G., Gambera, L., Moretti, E., \& Piomboni, P. (2001). Genetic sperm defects and consanguinity. Human Reproduction, 16(7), 1365-1371.

Bahmanyar, S., Kaplan, D. D., DeLuca, J. G., Giddings, T. H., O’Toole, E. T., Winey, M., Salmon, E. D., Casey, P. J., Nelson, W. J., \& Barth, A. I. (2008). $\beta$-Catenin is a Nek2 substrate involved in centrosome separation. Genes \& development, 22(1), 91-105.

Baltz, J. M., Williams, P. O., \& Cone, R. A. (1990). Dense fibers protect mammalian sperm against damage. Biology of reproduction, 43(3), 485-491.

Bhullar, B., Zhang, Y., Junco, A., Oko, R., \& van der Hoorn, F. A. (2003). Association of kinesin light chain with outer dense fibers in a microtubule-independent fashion. Journal of Biological Chemistry, 278(18), $16159-16168$

Bilić, J., Huang, Y. L., Davidson, G., Zimmermann, T., Cruciat, C. M., Bienz, M., \& Niehrs, C. (2007). Wnt induces LRP6 signalosomes and promotes dishevelled-dependent LRP6 phosphorylation. Science, 316(5831), 1619-1622.

Bimboim, H. C., \& Doly, J. (1979). A rapid alkaline extraction procedure for screening recombinant plasmid DNA. Nucleic acids research, 7(6), $1513-1523$ 
Bisgrove, B. W., \& Yost, H. J. (2006). The roles of cilia in developmental disorders and disease. Development, 133(21), 4131-4143.

Borg, C. L., Wolski, K. M., Gibbs, G. M., \& O'Bryan, M. K. (2010). Phenotyping male infertility in the mouse: how to get the most out of a 'non-performer'. Human reproduction update, 16(2), 205-224.

Brohmann, H., Pinnecke, S., \& Hoyer-Fender, S. (1997). Identification and characterization of new cDNAs encoding outer dense fiber proteins of rat sperm. Journal of Biological Chemistry, 272(15), 10327-10332.

Boddy, M. N., Howe, K., Etkin, L. D., Solomon, E., \& Freemont, P. S. (1996). PIC 1, a novel ubiquitin-like protein which interacts with the PML component of a multiprotein complex that is disrupted in acute promyelocytic leukaemia. Oncogene, 13(5), 971-982.

Bouchard, M. J., Dong, Y., McDermott, B. M., Lam, D. H., Brown, K. R., Shelanski, M., Bellvé, A. R., \& Racaniello, V. R. (2000). Defects in nuclear and cytoskeletal morphology and mitochondrial localization in spermatozoa of mice lacking nectin-2, a component of cell-cell adherens junctions. Molecular and cellular biology, 20(8), 2865-2873.

Brandt, R., \& Lee, G. (1993). Functional organization of microtubule-associated protein tau. Identification of regions which affect microtubule growth, nucleation, and bundle formation in vitro. Journal of Biological Chemistry, 268(5), 3414-3419.

Braun, R. E., Behringer, R. R., Peschon, J. J., Brinster, R. L., \& Palmiter, R. D. (1989). Genetically haploid spermatids are phenotypically diploid. Nature,337(6205), 373-376.

Burfeind, P., Belgardt, B., Szpirer, C., \& Hoyer-Fender, S. (1993). Structure and chromosomal assignment of a gene encoding the major protein of rat sperm outer dense fibres. European Journal of Biochemistry,216(2), 497-505.

Burfeind, P., \& Hoyer-Fender, S. (1991). Sequence and developmental expression of a mRNA encoding a putative protein of rat sperm outer dense fibers. Developmental biology, 148(1), 195-204. 
Burmester, S., \& Hoyer-Fender, S. (1996). Transcription and translation of the outer dense fiber gene (Odf1) during spermiogenesis in the rat. A study by in situ analyses and polysome fractionation. Molecular reproduction and development, 45(1), 10-20.

Calvin, H. I., Hwang, F. H. F., \& Wohlrab, H. (1975). Localization of zinc in a dense fiber-connecting piece fraction of rat sperm tails analogous chemically to hair keratin. Biology of reproduction, 13(2), 228-239.

Chang, M. C. (1951). Fertilizing capacity of spermatozoa deposited into the fallopian tubes. Nature, 168(168), 697-698

Chemes, H. E., Puigdomenech, E. T., Carizza, C., Olmedo, S. B., Zanchetti, F., \& Hermes, R. (1999). Acephalic spermatozoa and abnormal development of the head-neck attachment: a human syndrome of genetic origin. Human Reproduction, 14(7), 1811-1818.

Chemes, L. B., Glavina, J., Alonso, L. G., Marino-Buslje, C., de Prat-Gay, G., \& Sánchez, I. E. (2012). Sequence evolution of the intrinsically disordered and globular domains of a model viral oncoprotein. PloS one,7(10), e47661.

Chia, I. V., \& Costantini, F. (2005). Mouse axin and axin2/conductin proteins are functionally equivalent in vivo. Molecular and cellular biology, 25(11), 4371-4376.

Corbit, K. C., Shyer, A. E., Dowdle, W. E., Gaulden, J., Singla, V., \& Reiter, J. F. (2008). Kif3a constrains $\beta$-catenin-dependent Wnt signalling through dual ciliary and non-ciliary mechanisms. Nature Cell Biology, 10(1), 70-76.

Cowin, P., Kapprell, H. P., Franke, W. W., Tamkun, J., \& Hynes, R. O. (1986). Plakoglobin: a protein common to different kinds of intercellular adhering junctions. Cell, 46(7), 1063-1073.

Cui, W. (2010). Mother or nothing: the agony of infertility. Bull.World Health Organ. $88(12), 881-882$.

Deming, S. L., Nass, S. J., Dickson, R. B., \& Trock, B. J. (2000). C-myc amplification in breast cancer: a meta-analysis of its occurrence and prognostic relevance. British journal of cancer, 83(12), 1688. 
Donkor, F. F., Mönnich, M., Czirr, E., Hollemann, T., \& Hoyer-Fender, S. (2004). Outer dense fibre protein 2 (ODF2) is a self-interacting centrosomal protein with affinity for microtubules. Journal of cell science, 117(20), 4643-4651.

Drewes, G., Ebneth, A., \& Mandelkow, E. M. (1998). MAPs, MARKs and microtubule dynamics. Trends in biochemical sciences, 23(8), 307-311.

Fawcett, D. W., Anderson, W. A., \& Phillips, D. M. (1971). Morphogenetic factors influencing the shape of the sperm head. Developmental biology,26(2), 220-251.

Fawcett, D. W., \& McNutt, N. S. (1969). The ultrastructure of the cat myocardium I. Ventricular papillary muscle. The Journal of cell biology,42(1), 1-45.

Fawcett, D. W., \& Phillips, D. M. (1969). Observations on the release of spermatozoa and on changes in the head during passage through the epididymis. J Reprod Fertil Suppl, 6, 405-418.

Fawcett, D. W. (1975). The mammalian spermatozoon. Developmental biology, 44(2), 394-436.

Fawcett, D. W. (1958). The structure of the mammalian spermatozoon.International Review of Cytology, 7, 195-234.

Feinberg, A. P., \& Vogelstein, B. (1983). A technique for radiolabeling DNA restriction endonuclease fragments to high specific activity. Analytical biochemistry, 132(1), 6-13.

Fitzgerald, C. J., Oko, R. J., \& van der Hoorn, F. A. (2006). Rat Spag5 associates in somatic cells with endoplasmic reticulum and microtubules but in spermatozoa with outer dense fibers. Molecular reproduction and development, 73(1), 92-100.

Fontaine, J. M., Rest, J. S., Welsh, M. J., \& Benndorf, R. (2003). The sperm outer dense fiber protein is the 10th member of the superfamily of mammalian small stress proteins. Cell stress \& chaperones, 8(1), 62.

Friend, D. S., \& Fawcett, D. W. (1974). Membrane differentiations in freeze-fractured mammalian sperm. The Journal of cell biology,63(2), 641-664. 
Fumoto, K., Hoogenraad, C. C., \& Kikuchi, A. (2006). GSK-3 $\beta$-regulated interaction of BICD with dynein is involved in microtubule anchorage at centrosome. The EMBO journal, 25(24), 5670-5682.

Fumoto, K., Kadono, M., Izumi, N., \& Kikuchi, A. (2009). Axin localizes to the centrosome and is involved in microtubule nucleation. EMBO reports, 10(6), 606-613.

Gastmann, O., Burfeind, P., Güunther, E., Hameister, H., Szpirer, C., \& Hoyer-Fender, S. (1993). Sequence, expression, and chromosomal assignment of a human sperm outer dense fiber gene. Molecular reproduction and development, 36(4), 407-418.

Gerdes, J. M., Liu, Y., Zaghloul, N. A., Leitch, C. C., Lawson, S. S., Kato, M., Beachy, P. A., Beales, P., DeMartino, G. N., Fischer, S., Badano, J. L., \& Katsanis, N. (2007). Disruption of the basal body compromises proteasomal function and perturbs intracellular Wnt response. Nature genetics, 39(11), 1350-1360.

Goto, M., O'Brien, D. A., \& Eddy, E. M. (2010). Speriolin is a novel human and mouse sperm centrosome protein. Human reproduction, 25(8), 1884-1894.

Hadjihannas, M. V., Brückner, M., Jerchow, B., Birchmeier, W., Dietmaier, W., \& Behrens, J. (2006). Aberrant $\mathrm{Wnt} / \beta$-catenin signaling can induce chromosomal instability in colon cancer. Proceedings of the National Academy of Sciences, 103(28), 10747-10752.

Haidl, G., Becker, A., \& Henkel, R. (1991). Poor development of outer dense fibres as a major cause of tail abnormalities in the spermatozoa of asthenoteratozoospermic men. Human Reproduction, 6(10), 1431-1438.

Haimo, L. T., \& Rosenbaum, J. L. (1981). Cilia, flagella, and microtubules.The journal of cell biology, 91(3), 125s-130s.

Halder, G., \& Johnson, R. L. (2011). Hippo signaling: growth control and beyond. Development, 138(1), 9-22.

Hanahan, D. (1983). Studies on transformation of Escherichia coli with plasmids. Journal of molecular biology, 166(4), 557-580. 
Hishiya, A., \& Takayama, S. (2008). Molecular chaperones as regulators of cell death. Oncogene, 27(50), 6489-6506.

Hofferbert, S., Burfeind, P., Hoyer-Fender, S., Lange, R., Haldl, G., \& Engel, W. (1993). A homozygous deletion of 27 basepairs in the coding region of the human outer dense fiber protein gene does not result in a pathologic phenotype. Human molecular genetics, 2(12), 2167-2170.

Hong, W., \& Guan, K. L. (2012, September). The YAP and TAZ transcription co-activators: key downstream effectors of the mammalian Hippo pathway. InSeminars in cell \& developmental biology (Vol. 23, No. 7, pp. 785-793). Academic Press.

Hoyer-Fender, S., Burfeind, P., \& Hameister, H. (1995). Sequence of mouse Odf1 cDNA and its chromosomal localization: extension of the linkage group between human chromosome 8 and mouse chromosome 15. Cytogenetic and Genome Research, 70(3-4), 200-204.

Hoyer-Fender, S., Petersen, C., Brohmann, H., Rhee, K., \& Wolgemuth, D. J. (1998). Mouse Odf2 cDNAs consist of evolutionary conserved as well as highly variable sequences and encode outer dense fiber proteins of the sperm tail. Molecular reproduction and development, 51, 167-175.

Huang, P., Senga, T., \& Hamaguchi, M. (2007). A novel role of phospho- $\beta$-catenin in microtubule regrowth at centrosome. Oncogene, 26(30), 4357-4371.

Hüber, D., \& Hoyer-Fender, S. (2007). Alternative splicing of exon 3 b gives rise to ODF2 and Cenexin. Cytogenetic and genome research, 119(1-2), 68-73.

Imajo, M., Miyatake, K., Iimura, A., Miyamoto, A., \& Nishida, E. (2012). A molecular mechanism that links Hippo signalling to the inhibition of Wnt/ $\beta$ - catenin signalling. The EMBO journal, 31(5), 1109-1122.

Irons, M. J., \& Clermont, Y. (1982). Formation of the outer dense fibers during spermiogenesis in the rat. The Anatomical Record, 202(4), 463-471.

Irons, M. J., \& Clermont, Y. (1982). Kinetics of fibrous sheath formation in the rat spermatid. American Journal of Anatomy, 165(2), 121-130. 
Ishikawa, H., Kubo, A., Tsukita, S., \& Tsukita, S. (2005). Odf2-deficient mother centrioles lack distal/subdistal appendages and the ability to generate primary cilia. Nature cell biology, 7(5), 517-524.

Jho, E. H., Lomvardas, S., \& Costantini, F. (1999). A GSK3 $\beta$ phosphorylation site in axin modulates interaction with $\beta$-catenin and Tcf-mediated gene expression. Biochemical and biophysical research communications, 266(1), 28-35.

Jones, R. C. (1999). To store or mature spermatozoa? The primary role of the epididymis. International journal of andrology, 22(2), 57-66.

Joyner, A. L. (1993). Gene targeting: a practical approach. p 230. IRL Press, Oxford, United Kingdom.

Jürgens, G., Wieschaus, E., Nüsslein-Volhard, C., \& Kluding, H. (1984). Mutations affecting the pattern of the larval cuticle inDrosophila melanogaster. Wilhelm Roux's archives of developmental biology, 193(5), 283-295.

Kamal, A., Mansour, R., Fahmy, I., Serour, G., Rhodes, C., \& Aboulghar, M. (1999). Easily decapitated spermatozoa defect: a possible cause of unexplained infertility. Human Reproduction, 14(11), 2791-2795.

Kamitani, T., Kito, K., Nguyen, H. P., \& Yeh, E. T. (1997). Characterization of NEDD8, a developmentally down-regulated ubiquitin-like protein. Journal of Biological Chemistry, 272(45), 28557-28562.

Kaplan, D. D., Meigs, T. E., Kelly, P., \& Casey, P. J. (2004). Identification of a role for $\beta$-catenin in the establishment of a bipolar mitotic spindle. Journal of biological chemistry, 279(12), 10829-10832.

Kemler, R. (1993). From cadherins to catenins: cytoplasmic protein interactions and regulation of cell adhesion. Trends in Genetics, 9(9), 317-321.

Kerr, J. B., \& De Kretser, D. M. (1974). The role of the Sertoli cell in phagocytosis of the residual bodies of spermatids. Journal of reproduction and fertility, 36(2), 439-440.

Kierszenbaum, A. L., \& Tres, L. L. (2002). Bypassing natural sperm selection during fertilization: the azh mutant offspring experience and the alternative of 
spermiogenesis in vitro. Molecular and cellular endocrinology, 187(1), 133-138.

Kikuchi, A. (1999). Roles of Axin in the Wnt signalling pathway. Cellular signalling, 11(11), 777-788.

Kim, S. M., Choi, E. J., Song, K. J., Kim, S., Seo, E., Jho, E. H., \& Kee, S. H. (2009). Axin localizes to mitotic spindles and centrosomes in mitotic cells. Experimental cell research, 315(6), 943-954.

King, S. M. (2000). The dynein microtubule motor. Biochimica et Biophysica Acta (BBA)-Molecular Cell Research, 1496(1), 60-75.

Kishimoto, N., Cao, Y., Park, A., \& Sun, Z. (2008). Cystic Kidney Gene seahorse Regulates Cilia-Mediated Processes and Wnt Pathways.Developmental cell, 14(6), 954-961.

Klein, P. S., \& Melton, D. A. (1996). A molecular mechanism for the effect of lithium on development. Proceedings of the National Academy of Sciences,93(16), 8455-8459.

Kobayashi, T., \& Dynlacht, B. D. (2011). Regulating the transition from centriole to basal body. The Journal of cell biology, 193(3), 435-444.

Kohn, A. D., \& Moon, R. T. (2005). Wnt and calcium signaling: $\beta$-catenin-independent pathways. Cell calcium, 38(3), 439-446.

Kracklauer, M. P., Wiora, H. M., Deery, W. J., Chen, X., Bolival, B., Romanowicz, D., Simonette, R. A., Fuller, M. T., Fischer, J. A., \& Beckingham, K. M. (2010). The Drosophila SUN protein Spag4 cooperates with the coiled-coil protein Yuri Gagarin to maintain association of the basal body and spermatid nucleus. Journal of cell science,123(16), 2763-2772.

Lanneau, D., Brunet, M., Frisan, E., Solary, E., Fontenay, M., \& Garrido, C. (2008). Heat shock proteins: essential proteins for apoptosis regulation.Journal of cellular and molecular medicine, 12(3), 743-761.

Lapenta, V., Chiurazzi, P., van der Spek, P., Pizzuti, A., Hanaoka, F., \& Brahe, C. (1997). SMT3A, a Human Homologue of the S. cerevisiae SMT3 Gene, 
Maps to Chromosome 21qter and Defines a Novel Gene Family. Genomics, $40(2), 362-366$.

Lawrence, P. A., Struhl, G., \& Casal, J. (2007). Planar cell polarity: one or two pathways? Nature Reviews Genetics, 8(7), 555-563.

Lim, C. C., Lewis, S. E. M., Kennedy, M., Donnelly, E. T., \& Thompson, W. (1998). Human sperm morphology and in vitro fertilization: sperm tail defects are prognostic for fertilization failure. Andrologia, 30(1), 43-47.

Lindemann, C. B. (1996). Functional significance of the outer dense fibers of mammalian sperm examined by computer simulations with the geometric clutch model. Cell motility and the cytoskeleton, 34(4), 258-270.

Liu, C. Y., Zha, Z. Y., Zhou, X., Zhang, H., Huang, W., Zhao, D., Xiong, Y., Lei, Q. Y., \& Guan, K. L. (2010). The hippo tumor pathway promotes TAZ degradation by phosphorylating a phosphodegron and recruiting the SCF $\beta$-TrCP E3 ligase.Journal of biological chemistry, 285(48), 37159-37169.

Louie, R. K., Bahmanyar, S., Siemers, K. A., Votin, V., Chang, P., Stearns, T., Nelson, W. J., \& Barth, A. I. (2004). Adenomatous polyposis coli and EB1 localize in close proximity of the mother centriole and EB1 is a functional component of centrosomes. Journal of cell science, 117(7), 1117-1128.

Lupas, A., Van Dyke, M., \& Stock, J. (1991). Predicting coiled coils from protein sequences. Science, 252(5009), 1162-1164.

MacDonald, B. T., Tamai, K., \& He, X. (2009). Wnt/ $\beta$-catenin signaling: components, mechanisms, and diseases. Developmental cell, 17(1), 9-26.

Mahajan, R., Delphin, C., Guan, T., Gerace, L., \& Melchior, F. (1997). A small ubiquitin-related polypeptide involved in targeting RanGAP1 to nuclear pore complex protein RanBP2. Cell, 88(1), 97-107.

Mandelkow, E., \& Mandelkow, E. M. (1995). Microtubules and microtubule-associated proteins. Current opinion in cell biology, 7(1), 72-81.

Marmor, D., \& Grob-Menendez, F. (1991). Male infertility due to asthenozoospermia and flagellar anomaly: detection in routine semen analysis. International journal of andrology, 14(2), 108-116. 
Mardin, B. R., Lange, C., Baxter, J. E., Hardy, T., Scholz, S. R., Fry, A. M., \& Schiebel, E. (2010). Components of the Hippo pathway cooperate with Nek2 kinase to regulate centrosome disjunction. Nature cell biology, 12(12), 1166-1176.

Matunis, M. J., Coutavas, E., \& Blobel, G. (1996). A novel ubiquitin-like modification modulates the partitioning of the Ran-GTPase-activating protein RanGAP1 between the cytosol and the nuclear pore complex. The Journal of cell biology, 135(6), 1457-1470.

Matzuk, M. M., \& Lamb, D. J. (2008). The biology of infertility: research advances and clinical challenges. Nature medicine, 14(11), 1197-1213.

Miki, K., Qu, W., Goulding, E. H., Willis, W. D., Bunch, D. O., Strader, L. F., Perreault, S. D., Eddy E. M., \& O'Brien, D. A. (2004). Glyceraldehyde 3-phosphate dehydrogenase-S, a sperm-specific glycolytic enzyme, is required for sperm motility and male fertility. Proceedings of the National Academy of Sciences of the United States of America, 101(47), 16501-16506.

Morales, C. R., Oko, R., \& Clermont, Y. (1994). Molecular cloning and developmental expression of an mRNA encoding the $27 \mathrm{kDa}$ outer dense fiber protein of rat spermatozoa. Molecular reproduction and development, 37(2), 229-240.

Mortimer, S. T. (1997). A critical review of the physiological importance and analysis of sperm movement in mammals. Human Reproduction Update,3(5), 403-439.

McCREA, P. D., Turck, C. W., \& Gumbiner, B. (1991). A homolog of the armadillo protein in Drosophila (plakoglobin) associated with E-cadherin.Science, 254(5036), 1359-1361.

Murakami, M., Nakagawa, M., Olson, E. N., \& Nakagawa, O. (2005). A WW domain protein TAZ is a critical coactivator for TBX5, a transcription factor implicated in Holt-Oram syndrome. Proceedings of the National Academy of Sciences of the United States of America, 102(50), 18034-18039. 
Nebel, B. R., Amarose, A. P., \& Hackett, E. M. (1961). Calendar of gametogenic development in the prepuberal male mouse. Science, 134(3482), 832-833.

Nishida, T., Tanaka, H., \& Yasuda, H. (2000). A novel mammalian Smt3 - specific isopeptidase 1 (SMT3IP1) localized in the nucleolus at interphase. European Journal of Biochemistry, 267(21), 6423-6427.

Oko, R. (1988). Comparative analysis of proteins from the fibrous sheath and outer dense fibers of rat spermatozoa. Biology of reproduction, 39(1), 169-182.

Otani, H., Tanaka, O., Kasai, K. I., \& Yoshioka, T. (1988). Development of mitochondrial helical sheath in the middle piece of the mouse spermatid tail: regular dispositions and synchronized changes. The Anatomical Record,222(1), 26-33.

Oulad-Abdelghani, M., Bouillet, P., Décimo, D., Gansmuller, A., Heyberger, S., Dollé, P., Bronner, S., Lutz, Y., \& Chambon, P. (1996). Characterization of a premeiotic germ cell-specific cytoplasmic protein encoded by Stra8, a novel retinoic acid-responsive gene. The Journal of cell biology, 135(2), 469-477.

Ozawa, M., Baribault, H., \& Kemler, R. (1989). The cytoplasmic domain of the cell adhesion molecule uvomorulin associates with three independent proteins structurally related in different species. The EMBO Journal, 8(6), 1711.

Panda, D., Goode, B. L., Feinstein, S. C., \& Wilson, L. (1995). Kinetic stabilization of microtubule dynamics at steady state by tau and microtubule-binding domains of tau. Biochemistry, 34(35), 11117-11127.

Park, T. J., Mitchell, B. J., Abitua, P. B., Kintner, C., \& Wallingford, J. B. (2008). Dishevelled controls apical docking and planar polarization of basal bodies in ciliated epithelial cells. Nature genetics, 40(7), 871-879.

Pedersen, H. (1972). The postacrosomal region of the spermatozoa of man and Macaca arctoides. Journal of ultrastructure research, 40(3), 366-377.

Peifer, M., Berg, S., \& Reynolds, A. B. (1994). A repeating amino acid motif shared by proteins with diverse cellular roles. Cell, 76(5), 789-791.

Petersen, C., Füzesi, L., \& Hoyer-Fender, S. (1999). Outer dense fibre proteins from human sperm tail: molecular cloning and expression analyses of two 
cDNA transcripts encoding proteins of 70 kDa. Molecular human reproduction, 5(7), 627-635.

Porter, M. E., \& Sale, W. S. (2000). The 9+ 2 axoneme anchors multiple inner arm dyneins and a network of kinases and phosphatases that control motility. The Journal of cell biology, 151(5), F37-F42.

Ramos, A., \& Camargo, F. D. (2012). The Hippo signaling pathway and stem cell biology. Trends in cell biology, 22(7), 339-346.

Kierszenbaum, A. L., Rivkin, E., \& Tres, L. L. (2008). Expression of Fer testis (FerT) tyrosine kinase transcript variants and distribution sites of FerT during the development of the acrosome-acroplaxome-manchette complex in rat spermatids. Developmental Dynamics, 237(12), 3882-3891.

Rosales, J. L., Sarker, K., Ho, N., Broniewska, M., Wong, P., Cheng, M., van der Hoorn, F. A. \& Lee, K. Y. (2008). ODF1 phosphorylation by Cdk5/p35 enhances ODF1-OIP1 interaction. Cellular Physiology and Biochemistry, 20(5), 311-318.

Russell, L., \& Clermont, Y. (1976). Anchoring device between Sertoli cells and late spermatids in rat seminiferous tubules. The Anatomical Record,185(3), 259-277.

Russell, L. D., Ettlin, R. A., Hikim, A. P. S., \& Clegg, E. D. (1993). Histological and histopathological evaluation of the testis. International journal of andrology, 16(1), 83-83.

Russell, L. D., \& Malone, J. P. (1980). A study of Sertoli-spermatid tubulobulbar complexes in selected mammals. Tissue and Cell, 12(2), 263-285.

Russell, R. R. (1979). Glucan-binding Proteins of Streptococcus mutatis Serotype c. Journal of general microbiology, 112(1), 197-201.

Russell, S. D. (1984). Ultrastructure of the sperm of Plumbago zeylanica.Planta, 162(5), 385-391.

Salahshor, S., \& Woodgett, J. R. (2005). The links between axin and carcinogenesis. Journal of clinical pathology, 58(3), 225-236. 
Schalles, U., Shao, X., van der Hoorn, F. A., \& Oko, R. (1998). Developmental expression of the 84-kDa ODF sperm protein: localization to both the cortex and medulla of outer dense fibers and to the connecting piece. Developmental biology, 199(2), 250-260.

Schwarz-Romond, T., Metcalfe, C., \& Bienz, M. (2007). Dynamic recruitment of axin by Dishevelled protein assemblies. Journal of cell science, 120(14), 2402-2412.

Seifert, J. R., \& Mlodzik, M. (2007). Frizzled/PCP signalling: a conserved mechanism regulating cell polarity and directed motility. Nature Reviews Genetics, 8(2), 126-138.

Shao, X., Tarnasky, H. A., Lee, J. P., Oko, R., \& van der Hoorn, F. A. (1999). Spag4, a novel sperm protein, binds outer dense-fiber protein Odf1 and localizes to microtubules of manchette and axoneme. Developmental biology, 211(1), 109-123.

Shao, X., Tarnasky, H. A., Schalles, U., Oko, R., \& van der Hoorn, F. A. (1997). Interactional Cloning of the $84-\mathrm{kDa}$ Major Outer Dense Fiber Protein Odf84 leucine zippers mediate associations of Odf84 and Odf27. Journal of Biological Chemistry, 272(10), 6105-6113.

Shao, X., \& van der Hoorn, F. A. (1996). Self-interaction of the major 27-kilodalton outer dense fiber protein is in part mediated by a leucine zipper domain in the rat. Biology of reproduction, 55(6), 1343-1350.

Shen, Z., Pardington-Purtymun, P. E., Comeaux, J. C., Moyzis, R. K., \& Chen, D. J. (1996). UBL1, a human ubiquitin-like protein associating with human RAD51/RAD52 proteins. Genomics, 36(2), 271-279.

Simons, M., Gloy, J., Ganner, A., Bullerkotte, A., Bashkurov, M., Krönig, C., Schermer, B., Benzing, T., Cabello, O. A., Jenny, A., Mlodzik, M., Polok, B., Driever, W., Obara, T., \& Walz, G. (2005). Inversin, the gene product mutated in nephronophthisis type II, functions as a molecular switch between Wnt signaling pathways.Nature genetics, 37(5), 537-543. 
Slusarski, D. C., \& Pelegri, F. (2007). Calcium signaling in vertebrate embryonic patterning and morphogenesis. Developmental biology, 307(1), 1-13.

Smith, W. C., \& Harland, R. M. (1992). Expression cloning of noggin, a new dorsalizing factor localized to the Spemann organizer in Xenopus embryos. Cell, 70(5), 829-840.

Sokol, S., Christian, J. L., Moon, R. T., \& Melton, D. A. (1991). Injected Wnt RNA induces a complete body axis in Xenopus embryos. Cell, 67(4), 741-752.

Song, D. H., Dominguez, I., Mizuno, J., Kaut, M., Mohr, S. C., \& Seldin, D. C. (2003). CK2 phosphorylation of the armadillo repeat region of $\beta$-catenin potentiates Wnt signaling. Journal of Biological Chemistry, 278(26), $24018-24025$.

Soung, N. K., Kang, Y. H., Kim, K., Kamijo, K., Yoon, H., Seong, Y. S., Kuo, Y. L., Miki, T., Kim, S. R., Kuriyama, R., Giam, C. Z., Ahn, C. H., \& Lee, K. S. (2006). Requirement of hCenexin for proper mitotic functions of polo-like kinase 1 at the centrosomes. Molecular and cellular biology, 26(22), $8316-8335$

Southern, E. M. (1975). Detection of specific sequences among DNA fragments separated by gel electrophoresis. Journal of molecular biology,98(3), 503-517.

Sprando, R. L., \& Russell, L. D. (1987). A comparative study of Sertoli cell ectoplasmic specializations in selected non-mammalian vertebrates. Tissue and Cell, 19(4), 479-493.

Stackpole, C. W., \& Devorkin, D. (1974). Membrane organization in mouse spermatozoa revealed by freeze-etching. Journal of ultrastructure research,49(2), 167-187.

Sun, Y., \& MacRae, T. H. (2005). Small heat shock proteins: molecular structure and chaperone function. Cellular and Molecular Life Sciences CMLS, 62(21), 2460-2476.

Sun, Y., \& MacRae, T. H. (2005). The small heat shock proteins and their role in human disease. Febs Journal, 272(11), 2613-2627. 
Suzuki-Toyota, F., Ito, C., Toyama, Y., Maekawa, M., Yao, R., Noda, T., Iida, H., \& Toshimori, K. (2007). Factors maintaining normal sperm tail structure during epididymal maturation studied in $\mathrm{Gopc}^{-/-}$mice. Biology of reproduction, $77(1), 71-82$.

Takeichi, M. (1995). Morphogenetic roles of classic cadherins. Current opinion in cell biology, 7(5), 619-627.

Tanaka, H., Iguchi, N., Isotani, A., Kitamura, K., Toyama, Y., Matsuoka, Y., Onishi, M., Masai, K., Maekawa, M., Toshimori, K., Okabe, M., \& Nishimune, Y. (2005). HANP1/H1T2, a novel histone H1-like protein involved in nuclear formation and sperm fertility. Molecular and cellular biology, 25(16), 7107-7119.

Tang, X. M., Lalli, M. F., \& Clermont, Y. (1982). A cytochemical study of the Golgi apparatus of the spermatid during spermiogenesis in the rat. American Journal of Anatomy, 163(4), 283-294.

Thaler, C. D., \& Cardullo, R. A. (1995). Biochemical characterization of a glycosylphosphatidylinositol-linked hyaluronidase on mouse sperm.Biochemistry, 34(24), 7788-7795.

Tolwinski, N. S., \& Wieschaus, E. (2004). Rethinking WNT signaling.TRENDS in Genetics, 20(4), 177-181.

Toshimori, K. (1998). Maturation of mammalian spermatozoa: modifications of the acrosome and plasma membrane leading to fertilization. Cell and tissue research, 293(2), 177-187.

Turner, K. J., Sharpe, R. M., Gaughan, J., Millar, M. R., Foster, P. M., \& Saunders, P. T. (1997). Expression cloning of a rat testicular transcript abundant in germ cells, which contains two leucine zipper motifs. Biology of reproduction, 57(5), 1223-1232.

Tybulewicz, V. L., Crawford, C. E., Jackson, P. K., Bronson, R. T., \& Mulligan, R. C. (1991). Neonatal lethality and lymphopenia in mice with a homozygous disruption of the c-abl proto-oncogene. Cell, 65(7), 1153-1163. 
Van der Hoorn, F. A., Tarnasky, H. A., \& Nordeen, S. K. (1990). A new rat gene RT7 is specifically expressed during spermatogenesis. Developmental biology, 142(1), 147-154.

Vera, J. C., Brito, M., Zuvic, T., \& Burzio, L. O. (1984). Polypeptide composition of rat sperm outer dense fibers. A simple procedure to isolate the fibrillar complex. Journal of Biological Chemistry,259(9), 5970-5977.

Wantae, K., Minseong, K., \& Eek-hoon, J. (2013). Wnt/beta-catenin signalling: from plasma membrane to nucleus. Biochemical Journal, 450(1), 9-21.

Wijnhoven, B. P. L., Dinjens, W. N. M., \& Pignatelli, M. (2000). E-cadherin-catenin cell-cell adhesion complex and human cancer. British Journal of Surgery, 87(8), 992-1005.

Wolosewick, J. J., \& Bryan, J. H. (1977). Ultrastructural characterization of the manchette microtubules in the seminiferous epithelium of the mouse.American Journal of Anatomy, 150(2), 301-331.

Yamamoto, H., Kishida, S., Kishida, M., Ikeda, S., Takada, S., \& Kikuchi, A. (1999). Phosphorylation of axin, a Wnt signal negative regulator, by glycogen synthase kinase-3 $\beta$ regulates its stability. Journal of Biological Chemistry, 274(16), 10681-10684.

Yang, K., Meinhardt, A., Zhang, B., Grzmil, P., Adham, I. M., \& Hoyer-Fender, S. (2012). The small heat shock protein ODF1/HSPB10 is essential for tight linkage of sperm head to tail and male fertility in mice. Molecular and cellular biology, 32(1), 216-225.

Zamboni, L., \& Stefanini, M. (1971). The fine structure of the neck of mammalian spermatozoa. The Anatomical Record, 169(2), 155-172.

Zarsky, H. A., Cheng, M., \& van der Hoorn, F. A. (2003). Novel RING finger protein OIP1 binds to conserved amino acid repeats in sperm tail protein ODF1. Biology of reproduction, 68(2), 543-552.

Zeng, L., Fagotto, F., Zhang, T., Hsu, W., Vasicek, T. J., Perry Iii, W. L., Lee, J. J., Tilghman, S. M., Gumbiner, B. M \& Costantini, F. (1997). The Mouse 
Fused Locus Encodes Axin, an Inhibitor of the Wnt Signaling Pathway That Regulates Embryonic Axis Formation. Cell, 90(1), 181-192.

Zhao, B., Li, L., Tumaneng, K., Wang, C. Y., \& Guan, K. L. (2010). A coordinated phosphorylation by Lats and CK1 regulates YAP stability through SCF $\beta$-TRCP. Genes \& development, 24(1), 72-85. 


\section{Abbreviations}

\begin{tabular}{|l|l|}
\hline aa & Amino acid(s) \\
\hline Ab (Abs) & Antibody (antibodies) \\
\hline ALH & Amplitude of the lateral head displacement \\
\hline ANC-1 & Anchorage 1 protein \\
\hline APC & Adenomatous polyposis coli \\
\hline APMBT & Phenyl-benzothiazole \\
\hline APS & Ammonium persulfate \\
\hline ATP & Adenosine triphosphate \\
\hline BCF & Beat cross frequency \\
\hline bp & base pair(s) \\
\hline BSA & Bovine serum albumin \\
\hline Cdk5 & Cyclin-dependent kinase 5 \\
\hline cDNA & complementary DNA \\
\hline CK1 & Casein kinase 1 \\
\hline CK II & Casein kinase II \\
\hline Co-IP & Co-immunoprecipitation \\
\hline C-terminal & Carboxyl-terminal \\
\hline C-X-P & Cysteine-X-proline \\
\hline DAPI & 4',6 -diamidino-2-phenylindole \\
\hline dATP & Deoxyadenosine triphosphate \\
\hline deg. oocytes & degenerated oocytes \\
\hline DMEM & Dulbecco's Modified Eagle's Medium \\
\hline DMSO & Dimethyl sulfoxide \\
\hline DNA & Deoxyribonucleic acid \\
\hline Dnase & Deoxyribonuclease \\
\hline dpp & days post partum \\
\hline DTT & Dithiothreitol \\
\hline Dvl & Dishevelled \\
\hline ECFP & enhanced cyan fluorescent protein \\
\hline ECL & enhanced chemiluminescence \\
\hline E. coli & Escherichia coli \\
\hline EDTA & Ethylenediaminetetraacetic acid \\
\hline EM & Electron micrographs \\
\hline ER & Endoplasmic reticulum \\
\hline ES & Embryonic stem \\
\hline et al. & et alii (and others) \\
\hline FBS & Fetal bovine serum \\
\hline Fig & Figure \\
\hline fl & full length \\
\hline FS & Fibrous sheath \\
\hline
\end{tabular}




\begin{tabular}{|l|l|}
\hline Fz & Frizzled \\
\hline $\mathrm{g}$ & gram(s) \\
\hline x g (or RCF) & relative centrifugal force \\
\hline G418 & Geneticin \\
\hline GAPDH & Glyceraldehyde-3-Phosphate Dehydrogenase \\
\hline GFP & Green fluorescent protein \\
\hline Gopc & Golgi-associated PDZ- and coiled-coil motif-containing protein \\
\hline GSK3 & Glycogen synthase kinase 3 3 \\
\hline h & hour(s) \\
\hline HA & Human influenza hemagglutinin \\
\hline HANP1/H1T2 & Human Testis-specific H1 histone \\
\hline HCG & Human chorionic gonadotrophin \\
\hline HEK293 & Human embryonic kidney cell line \\
\hline HEPES & Hydroxyethyl piperazineethanesulfonic acid \\
\hline Hh & Hedgehog \\
\hline HPRT & Hypoxanthine phosphoribosyltransferase \\
\hline HRP & Horseradish peroxidase \\
\hline hSav1 & Salvador \\
\hline HSPB10 & Heat shock protein B10 \\
\hline HTCA & Head-to-tail coupling apparatus \\
\hline Hz & Hertz \\
\hline IFT & Intraflagellar transport \\
\hline IgG & Immunoglobulin G \\
\hline Inc. & Incorporated \\
\hline INM & Inner nuclear membrane \\
\hline IVF & in vitro fertilization \\
\hline K & Kinase \\
\hline KASH & Klarsicht/ANC-1/Syne-1 homology \\
\hline kb & kilobase \\
\hline kDa & kilodalton \\
\hline KLC3 & Kinesin light chain 3 \\
\hline ko & Knock out \\
\hline 1 & liter(s) \\
\hline LB & Lysogeny broth \\
\hline LEF & lymphoid enhancer factor \\
\hline LiAc & Lithium acetate \\
\hline LINC & Linker of nucleoskeleton and cytoskeleton \\
\hline LRP6 & Low density lipoprotein receptor-related protein 6 \\
\hline LSM & Confocal laser scanning microscopy \\
\hline M & molar \\
\hline mAb & mouse antibody \\
\hline MBP & maltose binding protein \\
\hline MBS & Modified Barth's Saline \\
\hline
\end{tabular}




\begin{tabular}{|c|c|}
\hline MCF7 & Michigan Cancer Foundation-7 \\
\hline $\mathrm{mg}$ & Milligram(s) \\
\hline $\min$ & Minute(s) \\
\hline MIS & Müllerian inhibiting substance \\
\hline $\mathrm{ml}$ & Millimeter(s) \\
\hline $\mathrm{mM}$ & Millimolar(s) \\
\hline MSP-300 & Muscle-specific protein 300 \\
\hline Mst2 & Mammalian sterile 20-like kinase 2 \\
\hline MTOC & Microtubule-organizing center \\
\hline NEAA & Nonessential amino acids \\
\hline Nek2A & Never in mitosis A-related kinase 2 \\
\hline ng & $\operatorname{nanogram}(\mathrm{s})$ \\
\hline $\mathrm{nM}$ & nanomolar \\
\hline $\mathrm{nm}$ & nanometer(s) \\
\hline N-terminal & Amino-terminal \\
\hline ODF & Outer dense fiber \\
\hline OF & Fopflash \\
\hline OIP1 & ODF1-interacting protein \\
\hline ONM & Outer nuclear membrane \\
\hline OT & Topflash \\
\hline p- & phospho- \\
\hline p35 & $35 \mathrm{kDa}$ protein \\
\hline PBS & Phosphate-buffered saline \\
\hline PCM & Pericentriolar material \\
\hline PCP pathway & Planar cell polarity pathway \\
\hline PCR & Polymerase chain reaction \\
\hline PDGF & Platelet-derived growth factor \\
\hline PFA & Paraformaldehyde \\
\hline pg & $\operatorname{picogram}(\mathrm{s})$ \\
\hline $\mathrm{pH}$ & Potentium hydrogenii \\
\hline PK & Protein kinase \\
\hline $\mathrm{PKC}$ & Protein kinase $\mathrm{C}$ \\
\hline PL-FITC & fluorescein isothiocyanate-labeled peanut lectin \\
\hline pmol & $\operatorname{picomol}(\mathrm{s})$ \\
\hline PMSF & Phenylmethanesulfonyl fluoride \\
\hline PMSG & Pregnant mare serum gonadotrophin \\
\hline rpm & revolutions per minute \\
\hline RPMI & Roswell Park Memorial Institute \\
\hline RT-PCR & reverse transcription-PCR \\
\hline $\mathrm{SD}$ & Standard Deviation \\
\hline SDS-PAGE & Sodium dodecyl sulfate polyacrylamide gel electrophoresis \\
\hline sec & second(s) \\
\hline SFRP & Secreted Fz-related protein \\
\hline
\end{tabular}




\begin{tabular}{|l|l|}
\hline sh & small hairpin or short hairpin \\
\hline SH3 & SRC homology 3 domain \\
\hline sHSPs & small heat shock proteins \\
\hline siRNA & small interfering RNA \\
\hline SPAG4 & Sperm associated antigen 4 \\
\hline SPAG4L & Sperm associated antigen 4-like \\
\hline SSC & mouse spermatogonial stem cells \\
\hline STR & straightness \\
\hline Stra8 & Stimulated by retinoic acid gene 8 \\
\hline SUN & Sad1p, UNC-84 domains \\
\hline SYCP3 & Synaptonemal complex protein 3 \\
\hline SYNE-1 & Synaptic nuclear envelope protein 1 \\
\hline Tab. & Table \\
\hline TAE & Tris-acetate-EDTA \\
\hline TAZ & Tafazzin \\
\hline TBST & Tris-buffered saline with Tween 20 \\
\hline TCF & T-cell factor \\
\hline Tcf4 & Transcription factor 4 \\
\hline TEMED & Tetramethylethylenediamine \\
\hline Thr & Threonine \\
\hline$\beta$-TrCP & $\beta$-transducin repeat-containing protein \\
\hline U & Unit \\
\hline UNC & UNCoordinated \\
\hline UV & Ultraviolet \\
\hline$\mu \mathrm{F}$ & microfarad \\
\hline$\mu$ l & microliter(s) \\
\hline$\mu \mathrm{M}$ & micromolar \\
\hline$\mu \mathrm{m}$ & micrometer(s) \\
\hline$\mu \mathrm{g}$ & microgram(s) \\
\hline V & volt \\
\hline VAP & average path velocity \\
\hline VCL & curvilinear velocity \\
\hline VSL & straight line velocity \\
\hline Wnt & int/Wingless \\
\hline wt & wild-type \\
\hline w/v & weight/volume \\
\hline YFP & Yellow fluorescent protein \\
\hline & \\
\hline
\end{tabular}




\section{Supplemental data of reporter gene assays}

8.1 The influence of over-expressed ODF2 on $\beta$-catenin activity using reporter gene assays in NIH3T3 and HEK293 cells

\begin{tabular}{|c|c|c|c|c|c|c|c|}
\hline \multicolumn{8}{|c|}{ NIH3T3 } \\
\hline & No DNA & $p h R L, O F$ & $p h R L, O T$ & $\begin{array}{c}\text { phRL, OT, } \\
\beta \text {-catenin }\end{array}$ & $\begin{array}{c}\text { phRL, OT, } \beta \text {-catenin, } \\
\text { 138NCGFP }\end{array}$ & $\begin{array}{c}\text { phRL, OT, } \beta \text {-catenin, } \\
\text { LiAc }\end{array}$ & $\begin{array}{c}\text { phRL, } O T, \beta \text {-catenin, } \\
\text { 138NCGFP, LiAc }\end{array}$ \\
\hline \multirow{3}{*}{ firefly reader } & 1378 & 7374 & 3351 & 7731 & 2866 & 23049 & 14646 \\
\hline & - & 5052 & 3223 & 7944 & 2892 & 27829 & 10768 \\
\hline & - & - & 2900 & 6141 & 2467 & 27565 & 11363 \\
\hline \multirow{3}{*}{ renilla reader } & 28441 & 57509562 & 40843648 & 25475516 & 21544719 & 12824167 & 9482575 \\
\hline & - & 45787517 & 40352438 & 30597611 & 21652981 & 14101061 & 8083070 \\
\hline & - & - & 37308972 & 28038856 & 18109621 & 14439908 & 9175368 \\
\hline
\end{tabular}

\begin{tabular}{|c|c|c|c|c|c|c|c|}
\hline \multicolumn{8}{|c|}{ HEK293 } \\
\hline & No DNA & $p h R L, O F$ & $p h R L, O T$ & $\begin{array}{c}\text { phRL, } O T, \\
\beta \text {-catenin }\end{array}$ & $\begin{array}{c}\text { phRL, } O T, \beta \text {-catenin, } \\
138 N C G F P\end{array}$ & $\begin{array}{c}\text { phRL, OT, } \beta \text {-catenin, } \\
\text { LiAc }\end{array}$ & $\begin{array}{c}\text { phRL, OT, } \beta \text {-catenin, } \\
\text { 138NCGFP, LiAc }\end{array}$ \\
\hline \multirow{3}{*}{ firefly reader } & 961 & 20753 & 16270 & 211473 & 89203 & 1009959 & 228679 \\
\hline & - & 19154 & 17980 & 248284 & 86838 & 1160628 & 169968 \\
\hline & - & - & 11780 & 143457 & 84167 & 808708 & 190763 \\
\hline \multirow{3}{*}{ renilla reader } & 18779 & 29108351 & 20304142 & 12056326 & 12741802 & 19926587 & 11099048 \\
\hline & - & 27400430 & 22385849 & 13308495 & 13984956 & 22309634 & 9381381 \\
\hline & - & - & 15517483 & 10491709 & 12734080 & 16499128 & 8250112 \\
\hline
\end{tabular}




\subsection{Canonical Wnt reporter gene assay using Odf 2 constructs}

\begin{tabular}{|c|c|c|c|c|c|c|c|c|c|c|c|c|c|c|}
\hline & $\begin{array}{l}\overleftrightarrow{z} \\
\stackrel{a}{a}\end{array}$ & $\begin{array}{l}\vdots \\
\vdots \\
\vdots \\
\vdots \\
\vdots\end{array}$ & $\begin{array}{l}5 \\
\vdots \\
\vdots \\
\vdots\end{array}$ & 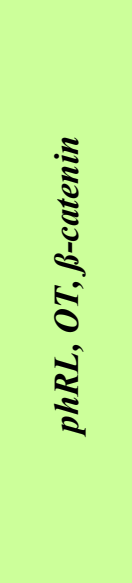 & 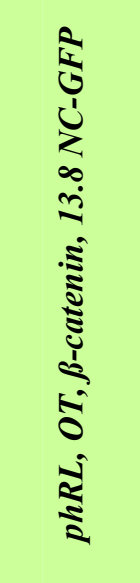 & 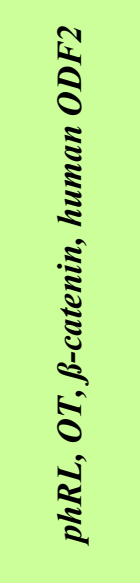 & 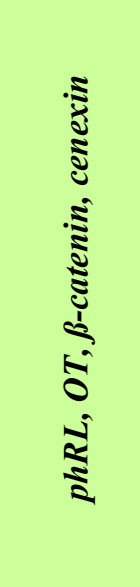 & 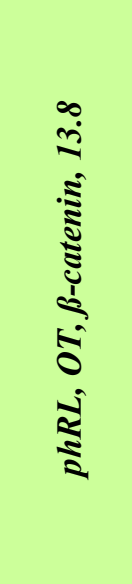 & 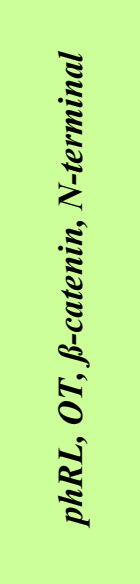 & 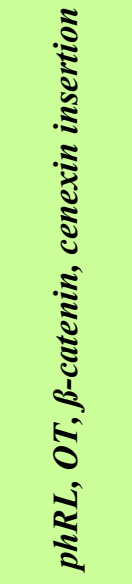 & 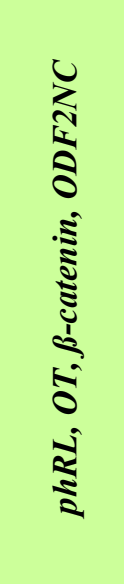 & 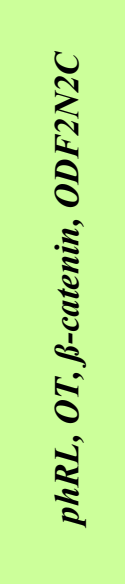 & 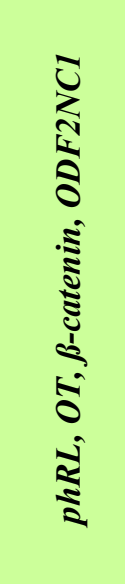 & 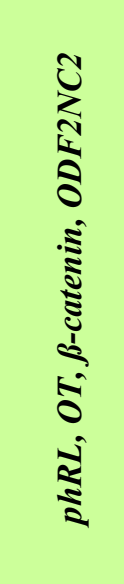 \\
\hline \multirow{3}{*}{$\begin{array}{l}\text { firefly } \\
\text { reader }\end{array}$} & 1556 & 727203 & 357278 & 3706065 & 1770570 & 2443493 & 513833 & 653735 & 2379942 & 1218055 & 617988 & 1479930 & 1059918 & 1000305 \\
\hline & 1352 & 566166 & 379842 & 3601263 & 2029647 & 2961604 & 828840 & 858302 & 2141210 & 1491183 & 598273 & 1402661 & 1111664 & 1291889 \\
\hline & 1225 & 584894 & 428492 & 3154732 & 1847670 & 3057815 & 932986 & 782598 & 2401239 & 1287619 & 639719 & 1091387 & 1013088 & 1571251 \\
\hline \multirow{3}{*}{$\begin{array}{l}\text { renilla } \\
\text { reader }\end{array}$} & 7893 & 12720777 & 8268050 & 5908044 & 6380253 & 5508231 & 2882880 & 3770576 & 4828445 & 4263478 & 6099054 & 7277764 & 5840538 & 4684783 \\
\hline & 6506 & 11674570 & 8782529 & 4036168 & 5272017 & 5100628 & 11701633 & 4134301 & 3674833 & 4529053 & 5347415 & 5709796 & 6678989 & 5796507 \\
\hline & 6634 & 15732937 & 10695043 & 5747074 & 6689655 & 7278079 & 6012836 & 5733381 & 6307252 & 6400197 & 5153624 & 5450932 & 5685871 & 7168344 \\
\hline
\end{tabular}


8.3 The influence of centrosomal and cytoskeletal proteins on canonical Wnt reporter

\begin{tabular}{|c|c|c|c|c|c|c|c|c|c|}
\hline & $\begin{array}{l}\frac{\pi}{2} \\
z \\
z\end{array}$ & $\begin{array}{l}5 \\
\vdots \\
\mathfrak{z}\end{array}$ & $\begin{array}{l}5 \\
\vdots \\
\vdots \\
\vdots\end{array}$ & 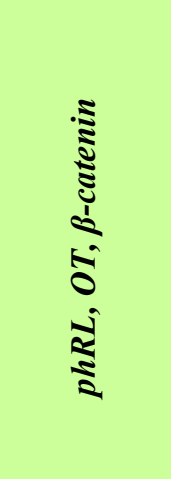 & 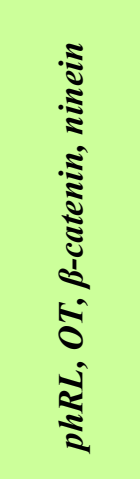 & 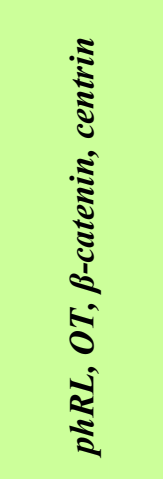 & 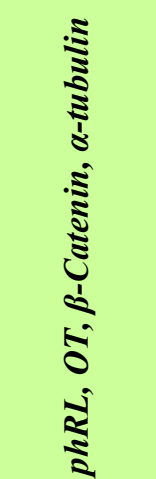 & 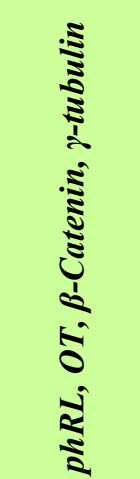 & 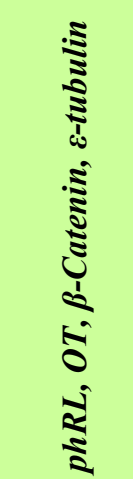 \\
\hline \multirow{3}{*}{$\begin{array}{l}\text { firefly } \\
\text { reader }\end{array}$} & 1727 & 2053181 & 2616769 & 11325074 & 5002019 & 5768227 & 269360 & 578413 & 4324384 \\
\hline & 1480 & 1897068 & 2724786 & 11259601 & 4789159 & 7442195 & 308977 & 613497 & 5371153 \\
\hline & 1293 & 1801418 & 2507826 & 12265290 & 4474195 & 6988264 & 322815 & 557159 & 5356482 \\
\hline \multirow{3}{*}{$\begin{array}{l}\text { renilla } \\
\text { reader }\end{array}$} & 11380 & 19722658 & 22747805 & 6617463 & 8806157 & 9087101 & 2079207 & 4790265 & 7399142 \\
\hline & 13328 & 18306177 & 26293198 & 7371483 & 8603418 & 11337738 & 2394214 & 5258110 & 8214671 \\
\hline & 13778 & 19948063 & 25515116 & 8149283 & 8478043 & 11268234 & 2786499 & 5060458 & 8931643 \\
\hline
\end{tabular}


8.4 The influence of centrosomal and cytoskeletal proteins on canonical Wnt reporter with or without nocodazole and taxol

\begin{tabular}{|c|c|c|c|c|c|c|c|c|c|c|c|c|c|c|c|}
\hline & $\begin{array}{l}\overleftrightarrow{z} \\
\stackrel{a}{a} \\
\varrho\end{array}$ & 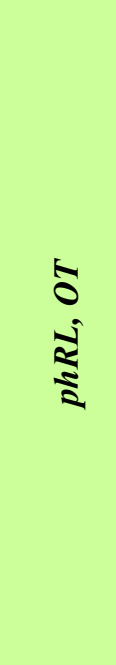 & $\begin{array}{l}5 \\
\vdots \\
\vdots \\
\vdots\end{array}$ & 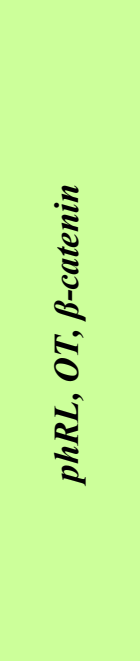 & 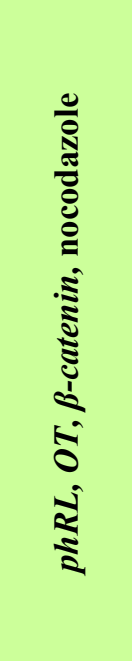 & 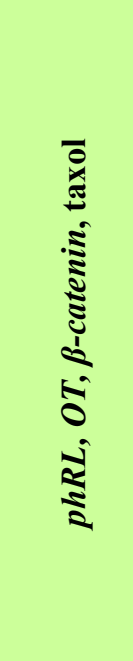 & 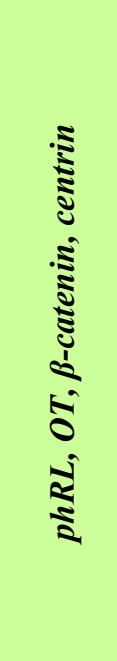 & 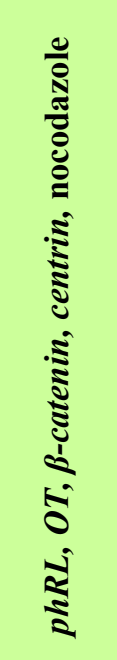 & 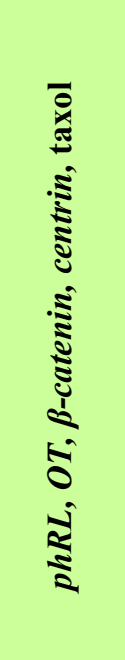 & 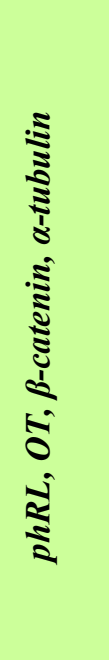 & 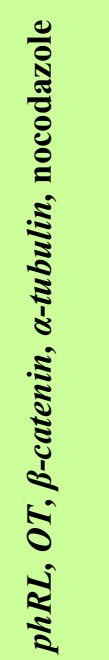 & 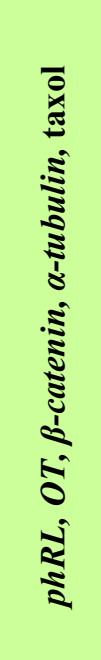 & 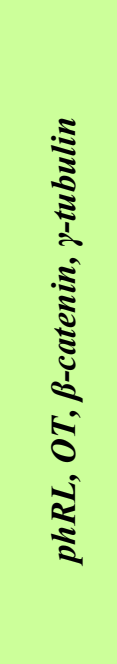 & 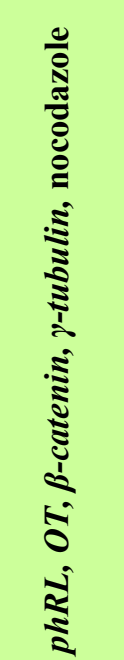 & 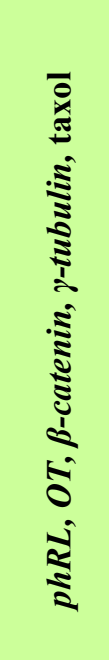 \\
\hline \multirow{3}{*}{$\begin{array}{l}\text { firefly } \\
\text { reader }\end{array}$} & 1922 & 2343863 & 1865420 & 30870594 & 27434451 & 27855739 & 4126153 & 4622874 & 6016341 & 255148 & 187021 & 324754 & 706663 & 514207 & 613523 \\
\hline & 1744 & 2072318 & 1500930 & 28118226 & 21216248 & 28061931 & 4995649 & 3888025 & 5082827 & 252443 & 217163 & 334952 & 626348 & 544673 & 795007 \\
\hline & 1667 & 2326734 & 1649516 & 35785426 & 14986673 & 27232861 & 6082622 & 4269483 & 5671293 & 228713 & 292817 & 375300 & 694543 & 801020 & 737758 \\
\hline \multirow{3}{*}{$\begin{array}{l}\text { renilla } \\
\text { reader }\end{array}$} & 6472 & $1.2 \mathrm{E}+07$ & 9620606 & 5703392 & 3585699 & 3341999 & 4230393 & 1770936 & 2449634 & 478749 & 356827 & 575657 & 2115175 & 1122236 & 1190226 \\
\hline & 6438 & $1.1 \mathrm{E}+07$ & 8070933 & 5244613 & 3323177 & 3251070 & 4295177 & 2078628 & 2137638 & 773710 & 443351 & 613472 & 2027657 & 1400594 & 1382980 \\
\hline & 5945 & $1.2 \mathrm{E}+07$ & 8549691 & 5542448 & 2075873 & 3314698 & 4702423 & 2153993 & 2163663 & 706000 & 522976 & 686293 & 2135341 & 1932101 & 1377596 \\
\hline
\end{tabular}




\section{GEORG-AUGUST-UNIVERSITÄT}

GÖTTINGEN

\section{Curriculum Vitae}

Name:

Last Degree:

Mailing Address:

\section{Kefei Yang}

Dipl

Department of Developmental Biology,

Johann-Friedrich-Blumenbach-Institute of Zoology and

Anthropology, Georg-August-University of Göttingen,

Justus-von-Liebig-weg 11

37077 Göttingen

E-mail Address: $\quad$ kyang@gwdg.de

\section{Academic Education:}

2009 - present: Doctoral study at Department of Developmental Biology,

Johann-Friedrich-Blumenbach-Institute of Zoology and

Anthropology, Göttingen Centre for Molecular Biosciences

(GZMB), Erst-Caspari-Haus, Georg-August-University

Göttingen, Germany

Research thesis supervised by Prof. Dr. Sigrid Hoyer-Fender.

Title of Thesis:

Functional Analysis of Two Major Sperm Tail Proteins Identifies ODF1 as Being Essential for the Tight Linkage of the Sperm Head to the Tail via SPAG4 and ODF2 as A Component of the $\beta$-catenin Destruction Complex 
2008 - 2009: $\quad$ Diploma thesis at Department of Developmental Biology,

Johann-Friedrich-Blumenbach-Institute of Zoology and

Anthropology, Göttingen Centre for Molecular Biosciences

(GZMB), Erst-Caspari-Haus, Georg-August-University

Göttingen, Germany

Research thesis supervised by Prof. Dr. Sigrid Hoyer-Fender.

Title of Thesis:

Expression Centrosomaler Proteine in Tumorzelllinien

2001 - 2008: $\quad$ Biology study, Georg-August-University

Göttingen, Germany

1999 - 2001: $\quad$ Learning German at AStA and Language Centre German

as a Foreign Language, Georg-August-University

Göttingen, Germany

1995 - 1999: $\quad$ Medicine study, Shenyang Medical School

Shenyang, Liaoning, P. R. China

\section{$\underline{\text { Publications }}$}

Yang, K., Meinhardt, A., Zhang, B., Grzmil, P., Adham, I. M., \& Hoyer-Fender, S. (2012). The small heat shock protein ODF1/HSPB10 is essential for tight linkage of sperm head to tail and male fertility in mice. Molecular and cellular biology, 32(1), 216-225.

Pletz, N., Medack, A., Rieß, E. M., Yang, K., Kazerouni, Z. B., Hüber, D., \& Hoyer-Fender, S. (2013). Transcriptional activation of Odf2/Cenexin by cell cycle arrest and the stress activated signaling pathway (JNK pathway).Biochimica et Biophysica Acta (BBA)-Molecular Cell Research, 1833(6), 1338-1346. 


\section{Zitaterklärung}

Die folgenden Kapitel meiner Dissertation, inklusive der Abbildungen und Tabellen, 1.1.4, 2.2.2.1, 2.2.2.2, 2.2.2.3, 2.2.2.4, 2.2.2.5, 2.2.2.6, 2.2.2.7, 2.2.2.8, 2.2.2.9, 2.2.2.11, 3.1.1.1, 3.1.1.2, 3.1.1.3, 3.1.1.4, 3.1.1.5, 4.1 sind im Wesentlichen aus

meiner eigenen Publikation „Yang, K., Meinhardt, A., Zhang, B., Grzmil, P., Adham, I. M., \& Hoyer-Fender, S. (2012). The small heat shock protein ODF1/HSPB10 is essential for tight linkage of sperm head to tail and male fertility in mice. Molecular and Cellular Biology, 32(1), 216-225" übernommen worden. 